\title{
Electrode Nanomaterials and Electrolytes for Aluminium-ion Batteries
}

by

Nicolò Canever

A thesis submitted to the Victoria University of Wellington in fulfilment of the requirements for the degree of Doctor of Philosophy in Chemistry. Victoria University of Wellington 



\section{Abstract}

Energy is one of the biggest challenges of the 21st century. Factors such as the decline in availability of non-renewable power sources, the alarming levels of atmospheric $\mathrm{CO}_{2}$, and the steady increase of the worldwide demand for energy make the worldwide transition to a fully renewable source-based production an extremely urgent necessity. Because of the intermittent nature of most renewable energy sources, battery-based energy storage systems could be a useful tool for such transition. However, current battery technologies such as lithium-ion often lack the cost-effectiveness and safety requirements necessary for large-scale grid energy storage applications; it is therefore important to search for alternative technologies which are more suitable for this purpose.

Aluminium-ion batteries have recently emerged as a very promising alternative to lithium-based systems, thanks to the low cost, non-flammability, and threeelectron redox chemistry of aluminium. Al-ion batteries could, in principle, offer better cost-effectiveness, higer capacity and improved safety, which would lead to a substantial advance in energy storage technology.

This $\mathrm{PhD}$ project deals with the investigation of novel electrode nanomaterials and electrolyte systems for Al-ion batteries. Particular emphasis is put on using the special properties of nanomaterials to improve the performance of batteries and on searching for low-cost compounds to be used as alternative electrolytes. Developing these areas will enhance the cost-effectiveness of the technology, and facilitate its commercial feasibility.

Vanadium oxide nanofibres and carbon nanofibres were initially tested as cathode materials. The performance of such cathodes, however, did not meet expectations: $\mathrm{V}_{2} \mathrm{O}_{5}$ nanofibres showed poor reversibility, short cycling life, and underwhelming specific capacity, while carbon nanofibres displayed a mostly capacitive, adsorption-based energy storage mechanism, with no significant ion intercalation taking place. Nevertheless, the tests performed on the latter material led to the discovery of the phenomenon of solid-electrolyte interphase formation: this process was investigated in depth and found to be mainly caused by the presence of defects on the surface of the nanofibres, favouring the decomposition of the electrolyte into insoluble species during the charging phase. 
Two composite materials were then tested as cathode candidates: solvothermallyprepared core-sheath $\mathrm{C} / \mathrm{V}_{2} \mathrm{O}_{5}$ nanofibres, and a layered nanostructured electrode. The former material showed an interesting behaviour as a battery cathode, as evidence for a multiple-ion intercalation mechanism was found; this phenomenon is however short-lived, as the cathode tends to disintegrate after the first few chargedischarge cycles. In a similar fashion, the fabrication methods used to create the layered electrode were shown to be unreliable: the poor adhesion of the active material to the underlying current collector resulted in highly unstable performance of the cathode, leading to the premature failure of the battery device.

Within alternative electrolytes, mixtures of inorganic and non-ionic organic compounds were studied. Eutectic mixtures of aluminium trichloride with acetamide and other small amide analogues were found to achieve good performance as battery electrolytes. Reduction of viscosity was found to be the key to improve cycling performance: this was achieved either by dilution of the electrolytes with an appropriate solvent, or by using combinations of amides to weaken the intermolecular interactions present in the melts. 


\section{Acknowledgments}

My journey through the creation of this thesis would not have been possible without the help and support of the people around me, from both professional and personal sides. I am very grateful to all, and hope to one day return the favour to each one of them.

On the academic side, I would like to acknowledge the support of my supervisors. Thomas Nann, for believing in me, giving me a chance to achieve this goal and providing financial support throughout the project. Jim Johnston, for accepting me into his research group with such short notice, while still prioritising my work and treating me the same way as all his other students. Renee Goreham, for also stepping in and providing much needed advice. Eric Le $\mathrm{Ru}$, for being a reliable and friendly secondary supervisor throughout most of the duration of this project. I am also thankful to all my past and present group mates, with whom I shared many brainstorming sessions, challenges, milestones, and all the frustrating and exciting moments that scientific research brings. In a rough order of appearance: Siobhan, Kathryn, Fraser, Rishabh, Vaibhav, Rohan, Shalini, Sam, Garima, Erin, Zeineb, Déanna, Hellen, Jacob. I wish a successful career to all of you. A special mention goes also to Geoffry Laufersky, for his valuable feedback during the writing of this thesis. Special thanks also go to visiting students Nicolas Bertrand and Stuart Oliver for contributing to some of the experimental work presented in this thesis (Chapter 8). A very well-earned thank you also goes to all the academic staff and technicians who trained me and assisted me with many of the analytical techniques and instrumentation used in this project. Among the instrument technicians, I would like to acknowledge David Flynn, Ian Vorster, Mathew Cairns, and Chris Lepper for their invaluable support throughout my research: you are truly the unsung heroes of academia. Thanks to Dr. Anna Henning for her training on TEM electron diffraction, Johan Grand for assisting me with Raman spectroscopy, and Teresa Gen for helping with FT-IR. Many thanks to the university workshop team for their kind assistance with various projects and instruments: Nick Grinter, 
Manu Pouajen-Blakiston, Alan Rennie, Alex Puglisi (mechanical workshop), Rodney Brown (electrical workshop), and Grant Franklin (glassblowing workshop). I would also like to acknowledge professors Andy Edgar, Grant Williams, Jonathan Halpert, Robin Fulton, Martyn Coles, and their research groups for training me and allowing me to use their instrumentation. Thanks also to Dr. Colin Doyle from the university of Auckland for his help with XPS experiments.

The help and support from my close friends and family is what really gave me the strength and motivation to complete this project; I feel incredibly lucky to be surrounded by such amazing and loving people. Thank you to my family: My father Roberto, my brother Matteo. You are the best family I could have ever wished for and I am honoured to be part of it. Thanks to my mother Sonia for raising me as the person I am now. I know you would be proud of me. A special mention goes to my partner Kate, who looked after me with unconditional love and care through all the hardships and tribulations of this project. I owe a lot of my success to you, and wish you the best of luck for your upcoming development. I am also thankful to all the amazing friends I met in this wonderful country, both within university and outside. Thanks to all of you for the good times and memories. In a rough order of appearance: Lars, Sofie, Line, Eva, Karoline, Claire, Amira, Justinas, Putri, Shyamal, Gianluca, Scotty, Davide, Valentina, Beatrice, Marco, Daniele, Jamie, Valentin, Thomas, Chriselle, Parth, Jordan, Joe, Olly, Mike, Ethan, Sasha, Isabella, Clement, Karen, Ronnie, Léo, Joe, Cam, Ella, Ben, Joey, Matt, Caitlin, Danielle, Emma, Kelsey, Will, Rosie, Gabriela, Alejandro, Oscar, Thomas. Finally, I could not finish this section without also mentioning my close friends back in Italy: I'm glad that geographical limitations were no match for our friendships, and I can still count you on my side after all this time. Enrico ("Busky"), Giacomo ("Nardin"), Matteo ("Trebbia"), Matteo ("Ciba"), Riccardo, Luca, Edoardo, (il signor) Giancarlo, Stefano, Gianluca, Riccardo Cibin, Marco, Geremia, Yasmin, Eleonora, Erica, Claudia, Elettra, Camilla, Giacomo ("Watt"), Enrico ("Bob"), Jacopo, Daniele, Nicola, Irene, Sofia, Alice, Angela. I wish you the happiest, most fulfilling life possible. 


\section{Contents}

1 Introduction 3

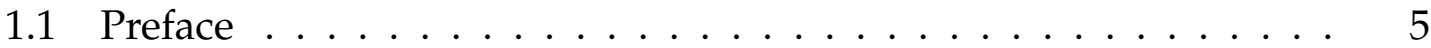

1.2 The intermittency of renewable energy sources . . . . . . . . 5

1.2.1 Batteries as large-scale energy storage . . . . . . . . . 6

1.2.2 Current state of lithium-ion batteries . . . . . . . . . . 7

1.3 Commercial alternatives to lithium-ion batteries . . . . . . . . . 9

1.3.1 Lead-acid batteries . . . . . . . . . . . . . . 9

1.3.2 Sodium-sulphur batteries ............... 10

1.3.3 Nickel-cadmium batteries . . . . . . . . . . . . . . . 11

1.3.4 Redox flow batteries (RFBs) . . . . . . . . . . . . . . . . . 12

1.4 Emerging battery technologies . . . . . . . . . . . . . 13

1.4.1 Aluminium-ion batteries . . . . . . . . . . . . . 15

1.5 Aim of the $\mathrm{PhD}$ thesis . . . . . . . . . . . . . . 17

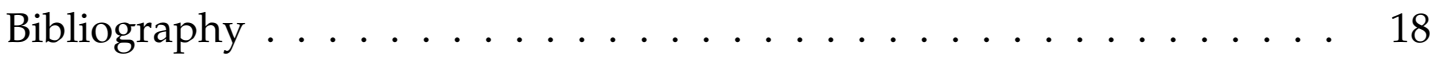

2 Literature review $\quad 21$

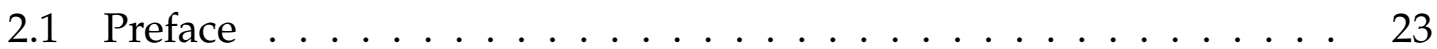

2.2 Background: Lithium-ion batteries . . . . . . . . . . . . . 23

2.3 History of the aluminium-ion battery . . . . . . . . . . . . . 24

2.4 Cathode materials . . . . . . . . . . . . . . . . 26

2.4 .1 Vanadium oxide .................. 26

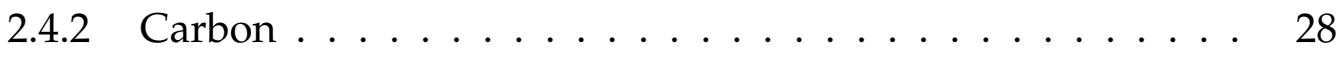

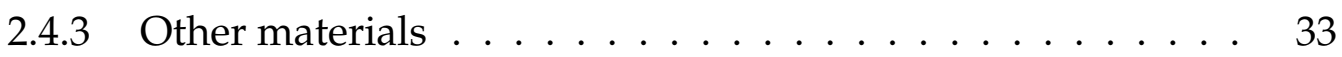

Polymers and small molecules . . . . . . . . . . . 33

Sulphur, selenium, and metal chalcogenides . . . . . . . 34

Other cathode materials . . . . . . . . . . . 36

2.5 Electrolytes . . . . . . . . . . . . . . . . 37 
2.5.1 Alkyl-imidazolium chloride melts . . . . . . . . . . 37

$\mathrm{AlCl}_{3} /[\mathrm{EMIm}] \mathrm{Cl}$ molar ratios . . . . . . . . . . . 38

2.5.2 Alternative electrolytes . . . . . . . . . . . . . . . 41

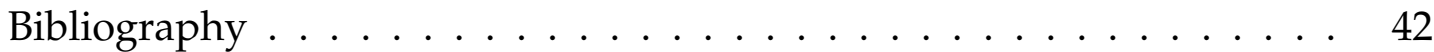

3 Methods and techniques $\quad 49$

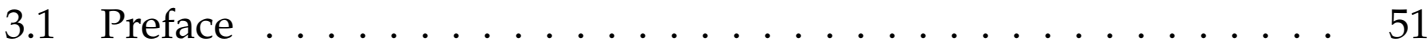

3.2 Nanomaterials fabrication techniques . . . . . . . . . . . 51

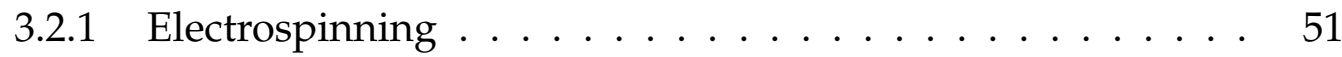

Coaxial electrospinning . . . . . . . . . . . 53

3.2.2 Hydrothermal and solvothermal synthesis . . . . . . . . 54

3.2.3 Thermal treatments . . . . . . . . . . . . . . . . . . . 54

3.3 Nanomaterials characterisation techniques . . . . . . . . . . 55

3.3.1 Electron microscopy . . . . . . . . . . . . . . . . . 55

3.3.2 X-ray diffraction (XRD) $\ldots \ldots \ldots \ldots \ldots$

3.3.3 Fourier transform infrared (FT-IR) spectroscopy . . . . . . 57

Raman spectroscopy . . . . . . . . . . . . . . . . . 58

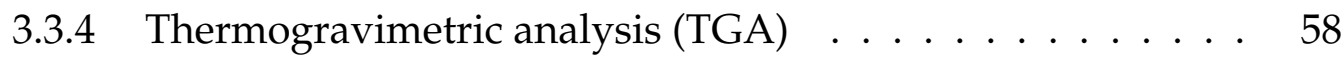

3.3.5 Brunauer-Emmett-Teller (BET) surface area measurement . 59

3.3.6 X-ray photoelectron spectroscopy (XPS) . . . . . . . . . . 59

3.4 Battery fabrication and testing . . . . . . . . . . . . . 60

3.4.1 Electrode fabrication . . . . . . . . . . . . . . . 60

3.4.2 Prototype battery devices . . . . . . . . . . . . . . . 61

3.4.3 Electrochemical testing . . . . . . . . . . . . . . . . . 63

Cyclic voltammetry . . . . . . . . . . . . . 63

Galvanostatic charge-discharge . . . . . . . . . . . . 65

Bibliography . . . . . . . . . . . . . . . . . 67

4 Cathode materials: $\mathrm{V}_{2} \mathrm{O}_{5}$ nanofibres $\quad 69$

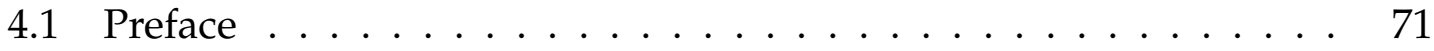

4.2 Nanofibre fabrication methods . . . . . . . . . . . . . . 71

4.2.1 Unsuccessful attempts . . . . . . . . . . . . . . . . 71

4.2.2 A novel fabrication method . . . . . . . . . . . . . 74

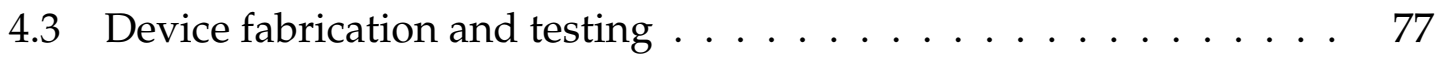

4.3.1 Electrode and cell fabrication . . . . . . . . . . . 77 


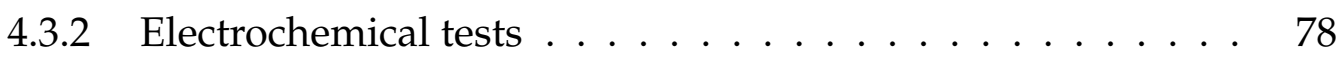

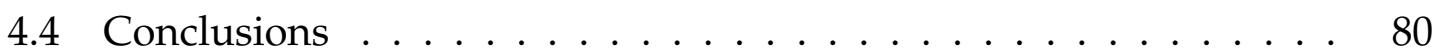

4.5 Experimental section $\ldots \ldots \ldots \ldots \ldots \ldots$

Bibliography . . . . . . . . . . . . . . . . 83

5 Cathode materials: carbon nanofibres $\quad 85$

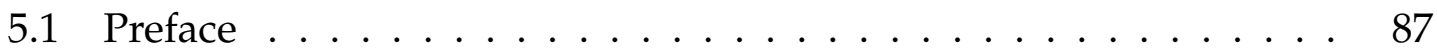

5.2 Polyacrylonitrile-based nanofibres $\ldots \ldots \ldots \ldots . \ldots 88$

5.2.1 Material preparation . . . . . . . . . . . . . . 88

5.2.2 Electrochemical tests . . . . . . . . . . . . . . . . . 92

Evidence for a solid-electrolyte interphase . . . . . . . 92

Galvanostatic cycling of CNFs . . . . . . . . . . . 105

5.3 Enhanced carbon nanofibres . . . . . . . . . . . . . . . . . 108

5.3.1 Efforts to improve nanofibre graphitisation . . . . . . . . . 109

5.3.2 Electrochemical tests . . . . . . . . . . . . . . . . . 114

5.4 Conclusions . . . . . . . . . . . . . . . . . . . 117

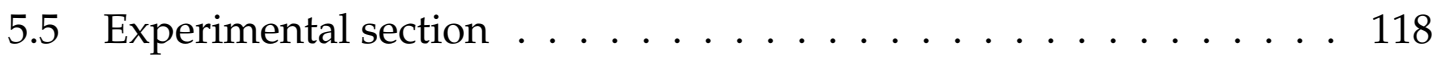

Bibliography . . . . . . . . . . . . . . . . . 121

6 Cathode materials: core-sheath $\mathrm{C} / \mathrm{V}_{2} \mathrm{O}_{5}$ nanofibres 125

6.1 Preface . . . . . . . . . . . . . . . . . . . 127

6.2 Core-sheath nanofibre fabrication methods . . . . . . . . . . 127

6.2.1 Coaxial electrospinning . . . . . . . . . . . . . . 127

6.2.2 Dip-coated CNF mats . . . . . . . . . . . . . . . 130

6.2.3 Solvothermal growth on CNFs . . . . . . . . . . . . 133

6.3 Electrochemical tests . . . . . . . . . . . . . . . . . . 138

6.3.1 The hypothesis of a mixed-ion intercalation mechanism . . . 139

6.4 Ex situ studies . . . . . . . . . . . . . . . . . . . . . . . . . . . 143

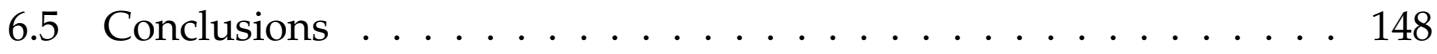

6.6 Experimental section . . . . . . . . . . . . . . . . . . . . . . 149

Bibliography . . . . . . . . . . . . . . . . . 152

7 Cathode materials: layered nanostructured electrode 155

7.1 Preface . . . . . . . . . . . . . . . . . . . 157

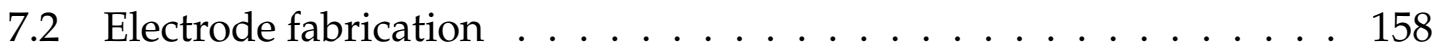


7.2.1 Nanostructured $\mathrm{V}_{2} \mathrm{O}_{5}$ layer . . . . . . . . . . . . 158

7.2.2 Electrospun separator ................. 161

7.3 Battery tests ........................ 161

7.3.1 Device assembly . . . . . . . . . . . . . 161

7.3.2 Electrochemical tests . . . . . . . . . . . . . . . 162

7.4 Conclusions . . . . . . . . . . . . . . . . . 164

7.5 Experimental section . . . . . . . . . . . . . . 165

Bibliography ........................... 168

8 Novel electrolytes: amides/ $\mathrm{AlCl}_{3}$ eutectic mixtures 169

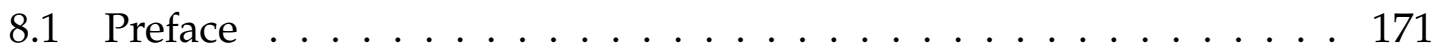

8.2 Acetamide-based electrolytes . . . . . . . . . . . . . . 172

8.2.1 The search for alternative compounds . . . . . . . . . . 172

8.2.2 Acetamide: preliminary tests ... . . . . . . . . 173

8.2.3 Diluted electrolytes . . . . . . . . . . . . . . 177

8.2.4 Investigating "ultrafast" cycling rates . . . . . . . . . . . 180

8.2.5 Swagelok-type cell tests ................ 181

8.3 Other amide $/ \mathrm{AlCl}_{3}$ eutectic mixtures . . . . . . . . . . . . . . . 184

8.3.1 Alternative amides as electrolytes . . . . . . . . . . . 184

8.3.2 Mixed amide electrolytes . . . . . . . . . . . . . . . 186

8.4 Conclusions . . . . . . . . . . . . . . . . . . . 189

8.5 Experimental section . . . . . . . . . . . . . . 190

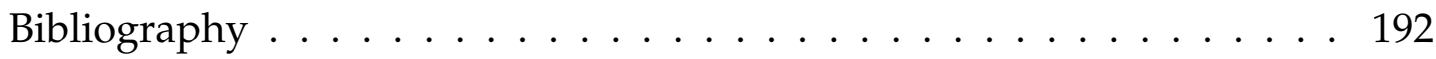

9 Conclusions and outlook $\quad 195$

9.1 Conclusions . . . . . . . . . . . . . . . 197

9.1.1 Nanostructured cathodes . . . . . . . . . . . . . . 197

9.1.2 Alternative electrolytes . . . . . . . . . . . . . 198

9.2 Future work . . . . . . . . . . . . . . . . . . . 198

$\begin{array}{ll}\text { A Supplementary data } & 201\end{array}$ 


\section{List of Figures}

1.1 Schematic of the working principle of an AIB $\ldots \ldots \ldots \ldots$

2.1 The Al- $\lambda-\mathrm{Mn}_{2} \mathrm{O}_{4}$ battery (from Paranthaman et al.) . . . . . . . 25

2.2 TEM Evidence of $\mathrm{Al}^{3+}$ intercalation into $\mathrm{V}_{2} \mathrm{O}_{5}$ (From Gu et al.) . . . 27

2.3 Galvanostatic cycling of zeolite-templated carbon (From Stadie et al.) 30

2.4 Electrolyte optimisation study (From Kravchyk et al.) . . . . . . . 32

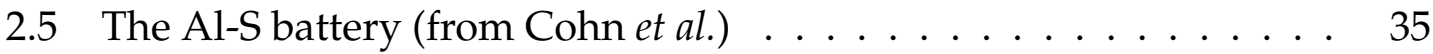

3.1 Diagram of the working principle of electrospinning . . . . . . 53

3.2 Design of a Swagelok cell . . . . . . . . . . . . . . . . 62

3.3 Examples of faradaic current and capacitive current in CV . . . . 64

4.1 SEM micrographs of failed $\mathrm{V}_{2} \mathrm{O}_{5} \mathrm{NFs}(1) \ldots \ldots \ldots \ldots \ldots \ldots$

4.2 SEM micrographs of failed $\mathrm{V}_{2} \mathrm{O}_{5} \mathrm{NFs}(2) \ldots \ldots \ldots \ldots \ldots$

4.3 SEM micrographs of $\mathrm{V}_{2} \mathrm{O}_{5} \mathrm{NFs} \ldots \ldots \ldots \ldots \ldots \ldots$

4.4 FT-IR spectra of $\mathrm{V}_{2} \mathrm{O}_{5} \mathrm{NFs} \ldots \ldots \ldots \ldots \ldots \ldots \ldots$

4.5 X-ray diffractogram of $\mathrm{V}_{2} \mathrm{O}_{5} \mathrm{NFs} \ldots \ldots \ldots \ldots \ldots$

4.6 Galvanostatic cycling of $\mathrm{V}_{2} \mathrm{O}_{5}$ AIBs . . . . . . . . . . . . . . . 79

5.1 SEM micrograph of graphitic foam (From Lin et al.) $\ldots \ldots \ldots 87$

5.2 Diagram of the PAN stabilisation reaction $\ldots \ldots \ldots \ldots$

5.3 SEM micrographs of CNFs . . . . . . . . . . . . . . . . . . . . 89

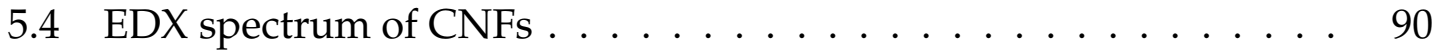

5.5 Thermogravimetric analysis of $\mathrm{CNFs} \ldots \ldots \ldots \ldots$

5.6 X-ray diffractograms of $\mathrm{CNFs} \ldots \ldots \ldots \ldots \ldots \ldots$

5.7 Charging profiles of $\mathrm{CNFs} \ldots \ldots \ldots \ldots$

5.8 SEM micrographs of charged CNFs . . . . . . . . . . . . . . 94

5.9 SEM-EDX spectrum and maps of charged CNFs . . . . . . . . 95

5.10 Cyclic voltammetry of pyrolytic graphite paper . . . . . . . . . 96 
5.11 XPS spectra of pristine and charged CNFs . . . . . . . . . . . 99

5.12 Cyclic voltammetry of CNFs . . . . . . . . . . . . . . . 103

5.13 Electrochemical testing of carbon film . . . . . . . . . . . 105

5.14 Galvanostatic cycling of CNFs . . . . . . . . . . . . . . . . . 106

5.15 "Ultrafast" galvanostatic cycling of CNFs . . . . . . . . . . . . . . 108

5.16 SEM micrographs of cobalt acetate-enhanced CNFs . . . . . . . . . 110

5.17 X-ray diffractograms of cobalt acetate-enhanced CNFs . . . . . . . . 111

5.18 Raman spectra of cobalt acetate-enhanced CNFs . . . . . . . . . . . 113

5.19 HRTEM images of cobalt acetate-enhanced CNFs . . . . . . . . . . 114

5.20 Cyclic voltammetry of cobalt acetate-enhanced CNFs . . . . . . . . 115

5.21 Galvanostatic cycling of cobalt acetate-enhanced CNF . . . . . . . . 116

6.1 Thermogravimetric analysis of PAN and PVP . . . . . . . . . . 128

6.2 SEM micrograph of coaxially electrospun precursor NF . . . . . . . 129

6.3 SEM micrographs of dip-coated CNFs . . . . . . . . . . . . . . . . 131

6.4 STEM-EDX maps of dip-coated CNFs annealed in air . . . . . . . . 131

6.5 STEM-EDX maps of dip-coated CNFs annealed under $\mathrm{N}_{2}$ atmosphere132

6.6 XRD of dip-coated CNFs after annealing under $\mathrm{N}_{2}$ atmosphere . . 133

6.7 SEM micrographs of solvothermal core-sheath NF (before annealing) 134

6.8 TGA of solvothermal core-sheath NF annealed in air . . . . . . . . . 135

6.9 STEM-EDX maps of solvothermal core-sheath NF annealed in air . 135

6.10 X-ray diffractogram of solvothermal core-sheath NF before annealing136

6.11 XRD of solvothermal core-sheath NF annealed under vacuum . . . 136

6.12 HRTEM micrograph of solvothermal core-sheath NF . . . . . . . . 137

6.13 STEM-EDX maps of vacuum-annealed solvothermal core-sheath NF 137

6.14 Galvanostatic cycling of core-sheath $\mathrm{C} / \mathrm{V}_{2} \mathrm{O}_{5} \mathrm{NF}$. . . . . . . . . 138

6.15 Galvanostatic cycling of core-sheath $\mathrm{C} / \mathrm{V}_{2} \mathrm{O}_{5} \mathrm{NF}$ (charge first) . . . 141

6.16 CV of core-sheath $\mathrm{C} / \mathrm{V}_{2} \mathrm{O}_{5} \mathrm{NF}$ before/after galvanostatic cycling . 143

6.17 Ex situ STEM-EDX maps of core-sheath $\mathrm{C} / \mathrm{V}_{2} \mathrm{O}_{5} \mathrm{NF}$. . . . . . . . . 144

6.18 Ex situ XPS depth profile of core-sheath $\mathrm{C} / \mathrm{V}_{2} \mathrm{O}_{5} \mathrm{NF} \ldots \ldots . . . . .146$

$6.19 \mathrm{SEM}$ of core-sheath $\mathrm{C} / \mathrm{V}_{2} \mathrm{O}_{5} \mathrm{NF}$ after galvanostatic cycling . . . . . 148

7.1 Schematic representation of the layered nanostructured electrode - 157

7.2 SEM micrograph of $\mathrm{V}_{2} \mathrm{O}_{5}$ nanoflowers . . . . . . . . . . . . . . 159

7.3 Issues with spin coating of $\mathrm{V}_{2} \mathrm{O}_{5}$ nanoflowers on CNFs $\ldots . . . . .160$ 
7.4 SEM micrograph and EDX maps of the assembled electrode . . . . 161

7.5 Galvanostatic cycling of the layered nanostructured electrode . . . 163

8.1 Diagram of $\mathrm{AcAm} / \mathrm{AlCl}_{3}$ reaction $\ldots \ldots \ldots \ldots \ldots \ldots$

8.2 Electroplating cyclic voltammograms of $\mathrm{AcAm} / \mathrm{AlCl}_{3}$ electrolytes . 174

8.3 Intercalation cyclic voltammograms of $\mathrm{AcAm} / \mathrm{AlCl}_{3}$ electrolytes . . 175

8.4 Galvanostatic cycling of $\mathrm{AcAm} / \mathrm{AlCl}_{3}$ electrolytes . . . . . . . 176

8.5 Galvanostatic cycling and voltage window of diluted AcAm/ $/ \mathrm{AlCl}_{3} 178$

8.6 Galvanostatic cycling of $\mathrm{AcAm} / \mathrm{AlCl}_{3}$ electrolytes $(r=1.5) \ldots \ldots$

8.7 Galvanostatic cycling of $\mathrm{AcAm} / \mathrm{AlCl}_{3}$ electrolytes at different rates 180

8.8 Galvanostatic cycling of $\mathrm{AcAm} / \mathrm{AlCl}_{3}$ in Swagelok cells . . . . . . 182

8.9 Galvanostatic cycling of diluted $\mathrm{AcAm} / \mathrm{AlCl}_{3}$ in Swagelok cells . . 183

8.10 Molecular structure of acetamide analogues . . . . . . . . . . . . 184

8.11 Galvanostatic cycling of acetamide analogue electrolytes . . . . . 185

8.12 Galvanostatic cycling of mixed amide electrolytes . . . . . . . . 186

8.13 Galvanostatic cycling of PrAm/AcAm electrolytes . . . . . . . . 188

8.14 Galvanostatic cycling of mixed amide electrolytes $(r=1.5) \ldots 189$

8.15 Digital image of three-electrode cell setup . . . . . . . . . . . 191

A.1 SEM micrographs of failed $\mathrm{V}_{2} \mathrm{O}_{5} \mathrm{NFs}$ (3) . . . . . . . . . . . 203

A.2 SEM micrographs of $\mathrm{V}_{2} \mathrm{O}_{5} \mathrm{NFs}$ from vanadium oxytriethoxide . . . 204

A.3 TEM micrograph of $\mathrm{V}_{2} \mathrm{O}_{5} \mathrm{NF} \ldots \ldots \ldots \ldots \ldots \ldots \ldots$

A.4 Galvanostatic cycling of $\mathrm{V}_{2} \mathrm{O}_{5}$ NFs with extended voltage window 205

A.5 SEM micrographs of failed PVP-based CNFs . . . . . . . . . . 205

A.6 Digital images of CNF and precursor NF mats . . . . . . . . 206

A.7 High magnification SEM micrographs of CNFs . . . . . . . . . 206

A.8 Galvanostatic cycling of pyrolytic graphite paper . . . . . . . . . 207

A.9 SEM and EDX of pyrolytic graphite paper after galvanostatic cycling 207

A.10 Raman spectra of CNFs _. . . . . . . . . . . . . . . . 208

A.11 Cyclic voltammograms of pyrolytic graphite paper . . . . . . . 208

A.12 Cyclic voltammogram of CNFs after a long charging step . . . . . 209

A.13 SEM micrographs of carbon film . . . . . . . . . . . . . . 209

A.14 TEM micrographs of CNFs . . . . . . . . . . . . . . . . 210

A.15 Galvanostatic cycling of ferrocene-enhanced CNFs . . . . . . . . . 210

A.16 SEM micrographs of annealed core-sheath $\mathrm{C} / \mathrm{V}_{2} \mathrm{O}_{5} \mathrm{NFs}$. . . . . . 211 
A.17 Galvanostatic cycling of core-sheath $\mathrm{C} / \mathrm{V}_{2} \mathrm{O}_{5}$ NFs (charge first) . 211

A.18 First galvanostatic cycle of AcAm $/ \mathrm{AlCl}_{3}$ electrolyte . . . . . . . . . 212

A.19 Cyclic voltammograms of diluted $\mathrm{AcAm} / \mathrm{AlCl}_{3}$ electrolytes . . . . 212

A.20 Specific capacities of diluted $\mathrm{AcAm} / \mathrm{AlCl}_{3}$ in Swagelok cells . . . . 213

A.21 Extended galvanostatic cycling of amide/ $\mathrm{AlCl}_{3}$ electrolyte . . . . . 213 


\section{List of Abbreviations}

$\begin{array}{ll}\text { AcAm } & \text { acetamide } \\ \text { AIB } & \text { aluminium-ion battery } \\ \text { ATR } & \text { attenuated total reflectance } \\ \text { BET } & \text { Brunauer-Emmett-Teller } \\ \text { BF } & \text { bright field } \\ \text { BMIm } & \text { 1-butyl-3-methylimidazolium } \\ \text { CE } & \text { counter electrode } \\ \text { CNF } & \text { carbon nanofibres } \\ \text { CV } & \text { cyclic voltammetry/voltammogram } \\ \text { CVD } & \text { chemical vapour deposition } \\ \text { DCM } & \text { dichloromethane } \\ \text { DMAc } & \text { N,N'-dimethylacetamide } \\ \text { DMF } & \text { N,N'-dimethylformamide } \\ \text { EDX } & \text { energy-dispersive X-ray } \\ \text { [EMIm] } & \text { 1-ethyl-3-methylimidazolium cation } \\ \text { FT-IR } & \text { Fourier transform infrared } \\ \text { HRTEM } & \text { high resolution transition electron microscopy } \\ \text { ICDD } & \text { International Centre for Diffraction Data } \\ \text { LIB } & \text { lithium ion battery } \\ \text { NF } & \text { nanofibre } \\ \text { Ni-MH } & \text { nickel-metal hydride } \\ \text { NMAc } & \text { N-methylacetamide } \\ \text { NMP } & \text { N-methyl-2-pyrrolidone } \\ \text { NMR } & \text { nuclear magnetic resonance } \\ \text { M } & \text { molecular weight } \\ \text { PAN } & \text { polyacrylonitrile } \\ \text { PDF } & \text { powder diffraction file } \\ \text { PEEK } & \text { polyether ether ketone } \\ \text { PMMA } & \text { poly(methyl methacrylate) } \\ \text { PrAm } & \text { propionamide } \\ \text { PTFE } & \text { polytetrafluoroethylene } \\ \text { PVDF } & \text { polyvinylidene fluoride } \\ \text { PVP } & \text { polyvinylpyrrolidone } \\ \text { RE } & \text { reference electrode } \\ \text { RFB } & \text { redox flow battery } \\ & \end{array}$




$\begin{array}{ll}\text { RSF } & \text { relative sensitivity factor } \\ \text { SAXS } & \text { small-angle X-ray scattering } \\ \text { SEI } & \text { solid-electrolyte interphase } \\ \text { SEM } & \text { scanning electron microscope/microscopy } \\ \text { SIB } & \text { sodium-ion battery } \\ \text { STEM } & \text { scanning transmission electron microscope/microscopy } \\ \text { TEM } & \text { transmission electron microscope/microscopy } \\ \text { TGA } & \text { thermogravimetric analysis } \\ \text { TOF-SIMS } & \text { time-of-flight secondary ion mass spectrometry } \\ \text { VO(acac) } & \text { vanadyl acetylacetonate } \\ \text { VOTIP } & \text { vanadium oxytriisopropoxide } \\ \text { WE } & \text { working electrode } \\ \text { XANES } & \text { X-ray absorption near edge structure } \\ \text { XRD } & \text { X-ray diffraction } \\ \text { XPS } & \text { X-ray photoelectron spectroscopy }\end{array}$


Chapter 1

\section{Introduction}





\subsection{Preface}

The following chapter will provide the broader context and motivation for undertaking this thesis. A brief introduction to the problem of intermittency of renewable energy sources will be outlined, followed by a description of the main commercially available and emerging battery-based energy storage systems.

\subsection{The intermittency of renewable energy sources}

Energy is arguably one of the biggest challenges of the $21^{\text {st }}$ century. Factors such as the decline in availability of non-renewable power sources, the alarming rise of atmospheric $\mathrm{CO}_{2}$ concentration levels, and the steady increase of the worldwide energy demand, especially from emerging economies, ${ }^{1}$ make the transition to a fully renewable source-based production of energy a very timely issue.

Renewable sources could potentially provide more than enough energy to satisfy the world demand: the sun alone outputs several times the amount of power to satisfy the requirements of the planet, $^{2}$ and other renewable sources such as wind, geothermal and tidal energy are similarly abundant and readily available. Furthermore, the cost per kilowatt of solar energy has been constantly decreasing in recent years, to the point of becoming really competitive with conventional sources such as coal or natural gas. ${ }^{3}$ Other renewable energy sources, such as wind or geothermal energy, are following similar trends and becoming very appealing for mass utilisation.

One of the biggest drawbacks of most renewable sources, however, is that their output is intermittent throughout the daily or seasonal cycle. The most glaring example of this can be found in solar energy: most of the energy coming from this source is output at midday; the peak demand for energy, on the other hand, is actually closer to sunset, when solar energy production is nearly zero. In this regard, conventional non-renewable energy sources have the clear advantage of producing energy in a continuous, dispatchable way. Furthermore, the output of these systems can also be modulated to satisfy the hourly fluctuations of grid load throughout the day. Because of this, output intermittency is perhaps the most important challenge that needs to be faced to switch over to a $100 \%$ renewable energy economy. Some measures can be taken to mitigate this problem, such 
as combining sources with different peak outputs to smooth out the daily supply (for example, solar power generation is highest at midday, whereas wind is often strongest at night and early morning), and utilising more dispatchable generation (hydroelectric, geothermal, biomass) to fill out the gaps when supply does not meet the energy demand. ${ }^{4}$ These methods alone, however, cannot solve the issue completely, and more robust strategies are therefore necessary. Perhaps the most effective response to the intermittency problem is to use energy storage technologies. These implementations allow the storage of any energy that is produced in excess so that it can be used at a later time, such as when generation is not enough to meet load. This would allow for a greater portion of renewable energy to be harvested and also ensure the continuity of energy availability. Several energy storage technologies currently exist, each with their own advantages and disadvantages.

\subsubsection{Batteries as large-scale energy storage}

One possible solution for energy storage is through the use of reversible electrochemical reactions in rechargeable batteries, which can then be discharged whenever needed to provide power to the grid. There are several advantages given by using this strategy for grid storage, and many of them arise because it is a wellestablished technology. First of all, this type of storage is easily scalable and can be applied to sites with different capacity requirements, from a few $\mathrm{kW}$ to the $\mathrm{MW}$ range. As a consequence, electrochemical batteries can even be implemented in fully independent, "off-grid" systems to power homes and personal equipment. Furthermore, thanks to the lack of moving parts, they are highly responsive and can change their output in minutes, rendering them also very suitable for accommodating variable demand peaks. Round-trip efficiencies of these systems can be as high as $95 \%,{ }^{5}$ depending mainly on the coulombic efficiency and energy efficiency of the electrochemical processes taking place in the battery. This puts batteries among the most efficient energy storage technologies in the market.

Generally speaking, an important metric for battery technologies is energy density, either gravimetric or volumetric. This is particularly important if the technol-

ogy is intended for mobile applications such as electric cars or portable electronic devices. On the other hand, grid energy storage is strictly a stationary application; 
therefore, there are no particular concerns regarding mass or volume limitations, and the important metric that instead needs to be considered is the price per $\mathrm{kWh}$. This implies that in order for batteries to be competitive against other energy storage solutions, they need to be built using inexpensive, abundant materials, so that the overall manufacturing cost remains as low as possible.

Currently, the most advanced commercially available battery technology is arguably the lithium-ion battery (LIB), based on the electrochemical intercalationdeintercalation reaction of lithium ions into host materials. Despite the relatively low abundance of lithium metal in the earth's crust, the recent increase in the production of plug-in electric vehicles has driven down the production costs of Li-ion batteries to below 300 US $\$ / \mathrm{kWh}$ (2015 data). ${ }^{6}$ Other technologies that are currently in use for electrical grid storage applications include lead acid, sodiumsulphur, and vanadium redox flow batteries. Each of these technologies has its own strengths and weaknesses, but lithium-ion is probably the most appealing at the moment due to its widespread application and extended research, which makes it a very mature and reliable technology.

\subsubsection{Current state of lithium-ion batteries}

Lithium-ion batteries were originally introduced in the 1970s, after the studies on reversible intercalation of lithium ions in graphite and other layered metal oxides. ${ }^{7}$ One of the most important breakthroughs for this technology was the discovery of the properties of lithium cobalt oxide $\left(\mathrm{Li}_{x} \mathrm{CoO}_{2}\right)$ as a cathode material by Goodenough's research group in 1980, ${ }^{8}$ in conjunction with the discovery of the graphite anode by Yazami and Touzain, ${ }^{9}$ in 1983. A more in-depth history of LIBs is outlined in Section 2.2. These developments led to the release of the first commercial rechargeable lithium-ion battery by Sony in 1991, which was initially implemented in mobile phones. The cell had a voltage higher than $3.6 \mathrm{~V}$ and a gravimetric energy density of $120-150 \mathrm{Wh} \mathrm{kg}^{-1}$. From there, much progress has been made in terms of energy density and cycle life: reports of state-of-the-art devices claim specific energy densities of up to $250 \mathrm{Wh} \mathrm{kg}^{-1}$ and lifetimes of up to $10,000$ cycles. Coulombic efficiencies are also very high ( $>95 \%)$, especially when compared to other technologies. ${ }^{5}$ Depending on the cathode materials used, the 
nominal cell voltage can vary between 3.2 and $3.8 \mathrm{~V}$, a notably high value, contributing to its attractive energy density.

For a battery using lithium cobalt oxide as cathode and graphite as anode, the working mechanism is based on the following two equations, written from a charged (left) to discharged (right) state:

Cathode: $\quad \mathrm{CoO}_{2}+\mathrm{Li}^{+}+\mathrm{e}^{-} \rightleftharpoons \mathrm{LiCoO}_{2}$

Anode: $\quad \mathrm{LiC}_{6} \rightleftharpoons \mathrm{C}_{6}+\mathrm{Li}^{+}+\mathrm{e}^{-}$

By combining these two equations, the cell reaction can be written as:

$$
\mathrm{LiC}_{6}+\mathrm{CoO}_{2} \rightleftharpoons \mathrm{C}_{6}+\mathrm{LiCoO}_{2}
$$

Although LIBs are quite a mature technology with a great deal of research and development on its shoulders, a few issues that might not make it the ideal solution for grid storage applications are still present. First of all, there are some economic concerns: although the price per $\mathrm{kWh}$ of LIBs has decreased notably in recent years, the availability of essential raw materials such as cobalt and lithium is relatively limited. It has been estimated that, if LIBs in their current state were to be adopted as the sole grid energy storage solution worldwide, requiring the production of 20 million batteries per year, the entire mining production of cobalt would be required, leading to the depletion of the total global cobalt reserves in less than 60 years. ${ }^{410}$ Furthermore, the increase in demand would likely increase the prices of the materials long before their availability would start to decrease. This is especially true for lithium: although the element currently constitutes only a small percentage of the overall price of the battery, ${ }^{*}$ an increase in demand caused by mass adoption of LIB-based grid energy storage could drive the prices up considerably and cause a dramatic increase of the production costs. The other big concern with LIBs is related to their safety. The cells are in some cases vulnerable to thermal runaway, ${ }^{11}, 12$ which happens when more heat is produced than what can be dissipated. This in turn can cause leaks, venting of gases, or in the worst of cases, fires or explosions. This becomes a major concern especially when many

\footnotetext{
* Only a small quantity of lithium is actually used in commercially available LIBs, in the form of a salt in the electrolyte
} 
cells are placed in close proximity to each other, such as in the case of grid storage stations. There are also a few drawbacks connected to the working principle of this battery architecture. One of them is the capacity fading significantly after a high number of cycles, which is mainly caused by the degradation of the electrode materials. ${ }^{13}$ This effect is made worse by higher temperatures. ${ }^{14}$ In addition, high temperature operation severely affects high-rate discharge capacities. For these reasons, in addition to the safety concerns, high temperatures (greater than $80^{\circ} \mathrm{C}$ ) need to be avoided at all costs. ${ }^{15}$ There are a few solutions being studied to overcome these issues, but they are either too expensive or have a negative effect on the durability advantages of LIBs. ${ }^{16}$

Due to the aforementioned reasons, it therefore is very important to investigate alternative battery chemistries to lithium-ion and find safer, more efficient, and cheaper technologies for grid energy storage.

\subsection{Commercial alternatives to lithium-ion batteries}

Although Li-ion is the most widespread technology in the market, a number of competing technologies are commercially available. Some of them have also been implemented in grid storage applications. The most relevant examples will be briefly described below.

\subsubsection{Lead-acid batteries}

This battery currently constitutes the standard technology used for powering automobile starter motors, thanks to its ability to provide high surge currents. The architecture features a lead dioxide $\left(\mathrm{PbO}_{2}\right)$ cathode, metallic lead as the anode, and uses a solution of sulphuric acid as the electrolyte. The working principle is based on a dissolution-precipitation mechanism, which follows the following two reactions:

Cathode: $\quad \mathrm{PbO}_{2}+3 \mathrm{H}^{+}+\mathrm{HSO}_{4}^{-}+2 \mathrm{e}^{-} \rightleftharpoons \mathrm{PbSO}_{4}+2 \mathrm{H}_{2} \mathrm{O}$

Anode:

$$
\mathrm{Pb}+\mathrm{HSO}_{4}^{-} \rightleftharpoons \mathrm{PbSO}_{4}+\mathrm{H}^{+}+2 \mathrm{e}^{-}
$$


By combining the two half-reactions, the overall cell equation for the discharge reaction can be written as such:

$$
\mathrm{PbO}_{2}+\mathrm{Pb}+2 \mathrm{H}_{2} \mathrm{SO}_{4} \rightleftharpoons 2 \mathrm{PbSO}_{4}+2 \mathrm{H}_{2} \mathrm{O}
$$

The nominal cell potential for this reaction is $2.1 \mathrm{~V}$. As shown by Equation 1.4, the electrolyte in lead-acid batteries is in fact an "active" material, as it participates in both the anodic and cathodic semi-reactions. Although this type of architecture uses inexpensive materials and can have high power densities, the low specific energy, low cycle durability, and the potential for gas evolution (overcharging can lead to the electrolysis of water and the generation of hydrogen and oxygen gases) makes lead-acid batteries a less-than-optimal solution for grid storage. Currently, this technology is only used for small-scale applications with low energetic requirements. ${ }^{17}$

\subsubsection{Sodium-sulphur batteries}

This technology was first developed by Ford Motor Company for powering early model electric vehicles. ${ }^{18}$ It utilises molten sodium metal as the anode, and elemental sulphur as the cathode. The two materials are separated by a solid $\beta$ alumina $\left(\beta-\mathrm{Al}_{2} \mathrm{O}_{3}\right)$ separator, which allows the transport of $\mathrm{Na}^{+}$ions, but is a poor electronic conductor. During the discharge process, $\mathrm{Na}^{+}$ions migrate to the cathode through the $\beta-\mathrm{Al}_{2} \mathrm{O}_{3}$ membrane; at the same time, electrons travel through the electrical circuit to the cathode to react with the sulphur and form sodium polysulphides $\mathrm{S}^{n-}$. The overall reaction for the discharge process is the following:

$$
2 \mathrm{Na}+\mathrm{xS} \rightleftharpoons \mathrm{Na}_{2} \mathrm{~S}_{\mathrm{x}}
$$

During the charge phase, this process is reversed. The cell potential for the above reaction is about $2 \mathrm{~V}$. The cell needs to operate at a high temperature (300-350 $\left.{ }^{\circ} \mathrm{C}\right)$ to keep the electrode materials in the liquid phase. During operation, the heat released by the electrochemical reactions is sufficient to maintain the elevated temperature, and no further heating needs to be supplied. Because of the high corrosiveness of the sodium polysulphides, cells are usually enclosed by an inert metal container and an air-tight alumina lid is also used to seal them and avoid 
any contact between the internal components the atmosphere. For this reason, the cost effectiveness of these cells increases with their size.

The advantages of Na-S batteries include high energy density, high coulombic efficiencies, and long cycle lives. Furthermore, the active materials are abundant and inexpensive, which imply low production costs and also make this an interesting alternative to Li-ion for energy storage. There are, however, a few hazard and design challenges that need to be overcome. First of all, the use of pure sodium metal presents a serious safety concern, as it can violently react with air and moisture. Another weak spot of this technology is given by the high temperature required for the battery to function; this is especially due to the poor mechanical properties of the ceramic electrolyte, which can get fractured from the thermally induced mechanical stress. This technology is already commercially available, and is currently used in several power storage facilities, the largest of which is situated in Buzen, Fukuoka Prefecture in Japan, and has a 300 MWh capacity and 50 MW power. ${ }^{19}$

\subsubsection{Nickel-cadmium batteries}

$\mathrm{Ni}-\mathrm{Cd}$ batteries are another relatively old technology, which has managed to remain relevant thanks to steady improvements over the years. The electrochemistry of Ni-Cd batteries is based on these two reactions (for the discharge phase):

$$
\begin{aligned}
& \text { Cathode: } \quad 2 \mathrm{NiO}(\mathrm{OH})+2 \mathrm{H}_{2} \mathrm{O}+2 \mathrm{e}^{-} \rightleftharpoons 2 \mathrm{Ni}(\mathrm{OH})_{2}+2 \mathrm{OH}^{-} \\
& \text {Anode: } \quad \mathrm{Cd}+2 \mathrm{OH}^{-} \rightleftharpoons \mathrm{Cd}(\mathrm{OH})_{2}+2 \mathrm{e}^{-}
\end{aligned}
$$

The nominal cell voltage achieved by this reaction is about $1.2 \mathrm{~V}$. It is important to distinguish between sealed Ni-Cd cells and vented wet cells: although nickelmetal hydride (Ni-MH) batteries ${ }^{\dagger}$ have replaced sealed $\mathrm{Ni}-\mathrm{Cd}$ cells for most consumer applications, vented cells are still advantageous, as they allow the release of any gas build-up generated by overcharging. This in turn allows for larger capacities and higher charge and discharge rates. Furthermore, it is a safer, cheaper

${ }^{\dagger}$ Nickel-metal hydride batteries use a similar electrochemical reaction to that of the Ni-Cd cell at the cathode, but a hydrogen-adsorbing metal alloy is used instead of cadmium at the anode. 
design, thanks to the release valve which negates any possibility of pressure buildup. They also have long lives and can be operated without complex management systems (except for occasional water replenishment) in a wide range of temperatures. Ni-Cd batteries were used in some of the earlier grid storage applications, ${ }^{20}$ but are now largely substituted by cheaper, safer, and better performing technologies.

\subsubsection{Redox flow batteries (RFBs)}

This technology features a slightly different working principle to the ones previously presented. Instead of using solid-state anodes and cathodes, chemical energy is stored in two liquid electrolyte solutions, containing two different redox couples, stored in two separate tanks. These solutions are pumped into a reactor, where they flow adjacent to each other, separated by an ion-selective membrane. During the discharge phase, one of the two electrolytes undergoes oxidation, giving out one electron to the electrical circuit; the redox species in the other electrolyte solution gets reduced, and hydrogen ions are exchanged through the membrane to complete the circuit. The inverse process takes place during the charging phase. This design can lead to a series of advantages over solid-state batteries: first of all, the "energy" and "power" components of the battery are physically separated. The former can be modulated by changing the quantity of electrolyte, and is dependent on the size of the storage tanks, while the latter is determined by the dimensions of the membrane. This distinction allows for batteries with flexible layouts and variable energy-to-power ratios, which can be tailored and adapted specifically to their target application. Furthermore, they have a considerably long cycle life, high over-discharging and overcharging tolerances, and require little maintenance. On the flip side, the most negative aspect of this technology is the generally low energy densities compared to solid-state technologies. Many different redox couples can be used in RFBs, each with its own advantages and disadvantages. One of the most promising and popular designs employs aqueous vanadium redox couples on both electrolytes, ${ }^{\ddagger}$ which has typically an open circuit voltage of about $1.4 \mathrm{~V}$. The first working prototype of this battery

\footnotetext{
${ }^{\ddagger}$ The $\mathrm{V}^{2+} / \mathrm{V}^{3+}$ couple is used in the negative electrode, and the $\mathrm{V}^{5+} / \mathrm{V}^{4+}$ is used in the positive electrode.
} 
was developed by the University of New South Wales in the 1980s ${ }^{21}$ The use of vanadium complexes in both the electrolytes is especially advantageous, as any cross-contamination between the two liquids does not result in permanent damage in the battery. The relatively high abundance of vanadium in the earth's crust $^{22}$ and the $1.4 \mathrm{~V}$ open circuit potential also make it quite attractive for grid storage applications.

There are still a few limitations in the technology due to the chemical stress imposed to the components of the electrochemical cell by the highly reactive $\mathrm{V}^{5+}$

species and the high acidity of the electrolyte solutions. Cross-permeation of the vanadium ions also presents a challenge, as it can lead to capacity losses, and thus expensive ion-transport membranes need to be used. Nevertheless, redox flow batteries utilising this chemistry are already commercially available and have been implemented in several sites to control the energy production from intermittent sources.

\subsection{Emerging battery technologies}

A great amount of research is being conducted in the field of battery science to develop better, more economical alternatives to lithium-ion batteries with the application of grid energy storage in mind. Although some of these efforts trace back to several decades ago, none of the technologies described hereafter have widespread commercial applications.

\section{Sodium-ion batteries}

Currently, sodium-ion batteries (SIBs) are one of the most studied options for substituting Li-ion. They employ a rocking-chair mechanism, similar to their lithiumion counterparts, with $\mathrm{Na}^{+}$ions reversibly intercalating into the positive and negative electrodes. The use of a different intercalating alkali ion in the battery chemistry leads to a series of advantages. Primarily, sodium is significantly cheaper and more abundant than lithium, which should bring about lower production costs. Furthermore, the redox potential of sodium is slightly lower than lithium (-2.7 $\mathrm{V}$ vs. standard hydrogen electrode, against $-3.0 \mathrm{~V}$ for lithium), which could also be beneficial to costs, as it allows the use of cheaper electrolytes, that often have 
narrower voltage windows. In terms of energy and power densities, it is unlikely that Na-ion technology will surpass Li-ion batteries due to the greater equivalent weight of $\mathrm{Na}$ and its larger ionic diameter. Both of these make the intercalation reaction less favourable for sodium ions than for lithium, despite the lower ionisation potential. ${ }^{23}$ Nevertheless, for large-scale storage applications, specific energy is not a fundamental characteristic, and production costs are definitely more relevant. Therefore, the aforementioned features, make sodium-ion a technology worth investigating.

Most research in SIBs is oriented towards the search for new compatible electrode materials and electrolytes. Because of the higher ionic diameter of sodium, not all electrode materials used in Li-ion batteries work for Na-ion as well. As far as anode materials are concerned, carbon seems to be the most popular material; particular emphasis in recent literature has been put on "disordered" carbons, which are believed to facilitate the intercalation reaction. ${ }^{24}$ Various materials have been proposed as candidates for the cathode, with phosphate-based materials showing overall the best performance thanks to their thermal stability and high voltage. ${ }^{25,26}$

\section{Magnesium-ion batteries}

Magnesium-based battery systems have also recently gained notable popularity as a potential grid energy storage technology. The working principle is also based on the reversible intercalation of $\mathrm{Mg}^{2+}$ ions into two-dimensional layered materials. There are economic advantages in this case as well, thanks to abundance of magnesium in the earth's crust. In addition, each $\mathrm{Mg}$ atom can give up two electrons in a redox reaction, corresponding to a rather high theoretical specific capacity (2205 $\left.\mathrm{Ah} \mathrm{kg}^{-1}\right) .{ }^{27}$ Compared to lithium and sodium, the metal is also more chemically stable, as it does not react with air or atmospheric moisture: this also allows metallic magnesium to be used as a negative electrode without any major safety concerns.

Commercially, the biggest difficulties that this technology must overcome concern finding electrolytes with a better practical application and developing low cost electrodes with long cycle lives. The choice of electrolyte in these systems is especially challenging, as it is necessary to find an electrolyte that avoids the formation of a passivation layer on the anode surface, preventing cycling of the 
battery. ${ }^{28}$ Furthermore, the double charge of the magnesium ion also adds the challenge of increasing the viscosity of the electrolyte solution, and has a negative effect on the charge mobility. ${ }^{29}$ The most viable options investigated so far are Grignard-like compounds in ether solutions, or gel-type polymers containing Mg species. ${ }^{30}$ Regarding cathode materials, the challenge lies in finding a material which can facilitate the slow solid-state diffusion of the $\mathrm{Mg}^{2+}$ ion. The most promising alternatives currently include "Chevrel phase" molybdenum chalcogenides and their derivatives. ${ }^{31,32}$

\subsubsection{Aluminium-ion batteries}

One of the most promising emerging battery technology is aluminium-ion batteries (AIBs). Aluminium is a highly abundant, inexpensive material, which is already used in numerous applications in different fields of engineering. It is a nontoxic metal, and is relatively inert under normal atmospheric conditions, thanks to its inherent $\mathrm{Al}_{2} \mathrm{O}_{3}$ passivating layer. The electrochemistry of AIBs is based on the $\mathrm{Al}^{0} / \mathrm{Al}^{3+}$ redox couple, where three electrons are therefore exchanged for every atom that gets oxidised or reduced. Thanks to this, aluminium anodes have one of the largest theoretical specific capacities in their field $\left(2980 \mathrm{Ah} \mathrm{Kg}^{-1}\right)$. Furthermore, the ionic radius of $\mathrm{Al}^{3+}$ is $53.5 \mathrm{pm}$, significantly smaller than $\mathrm{Li}^{+}(76 \mathrm{pm})$, which makes it a suitable candidate for intercalation.

AIBs operate through the reversible electroplating of $\mathrm{Al}$ metal at the anode. This is made possible by an ionic liquid electrolyte, which is generally composed of a eutectic mixture of $\mathrm{AlCl}_{3}$ and an organic chloride salt from the alkyl-imidazolium halogenide family. Such liquids have Lewis acid characteristics, and can effectively strip and plate $\mathrm{Al}^{3+}$ ions from a metallic aluminium anode. At the cathode, mechanisms involving conversion reactions or of reversible insertion of $\mathrm{Al}^{\mathrm{III}}$ containing ions into a suitable cathode are involved (Figure 1.1). It is worth noting that other types of aluminium-based battery electrochemistries also exist and are currently being investigated, such as aluminium-air batteries ${ }^{33-35}$ and aqueous AIBs. ${ }^{36-38}$ Such technologies, however, go beyond the scope of this thesis, and therefore will not be discussed herein.

Although this research topic is fairly new (the first relevant publication dates 


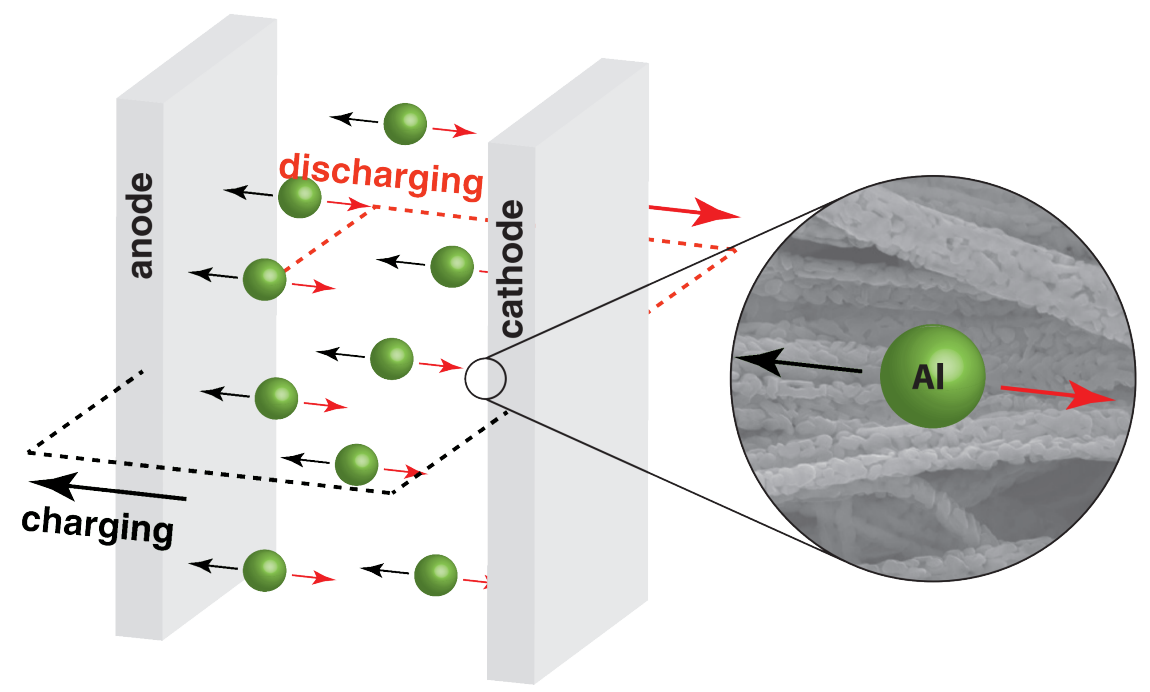

FIGURE 1.1: Schematic representation of the working principle of aluminium-ion batteries.

back to 2010), ${ }^{39}$ some of the early reports show a lot of potential, with publications proposing batteries with high specific capacities, ${ }^{40}$ high discharge voltages, ${ }^{41}$ "ultrafast" charge/discharge rates with high coulombic efficiencies and long cycling life. ${ }^{42}$ Most research efforts on AIBs are currently focused on investigating new cathode materials and electrolytes, with the aim of improving performance and reducing costs. Carbonaceous and graphitic materials seem to have attracted a lot of attention as cathode materials, thanks to their high cycling stability and fast charging capabilities. ${ }^{42}$ Vanadium (V) oxide also appears to be a very promising option, as it appears to provide excellent specific capacity. ${ }^{43}$ The predominant electrolyte system used in AIBs is a mixture of $\mathrm{AlCl}_{3}$ and 1-ethyl3-methylimidazolium $\left([\mathrm{EMIm}]^{+}\right.$) chloride. In terms of alternative electrolyte research, various organic and inorganic compounds have been studied as a substitute to the expensive alkyl-imidazolium chlorides: generally speaking, the ability to form a room temperature liquid when mixed with $\mathrm{AlCl}_{3}$ is a prerequisite for their use as an electrolyte candidate. Currently, no literature describing alternatives to metallic $\mathrm{Al}$ as anode has been published. This is justified by the fact that bulk aluminium metal works reasonably well as a negative electrode, yielding good cycling performance and no apparent issues deriving from dendrite growth, ${ }^{44}$ such as in the case of LIBs. A full review of the history and state of the art of non-aqueous AIBs is presented in Chapter 2. 
Because of all the advantages described above, AIBs are indeed a promising technology, which could be a very valid substitute for the current large-scale grid energy storage technologies. In addition, many of their features, such as the theoretically high specific capacity and fast charging rates, could also make this an attractive technology for portable applications.

\subsection{Aim of the PhD thesis}

This thesis aims to investigate new nanostructured cathode materials and electrolytes for AIBs. For the study of cathode materials, the expertise in the field of inorganic nanomaterials acquired by this research group was applied to the field of aluminium batteries in an attempt to enhance their performance. In current commercial batteries, electrode materials are mostly based on bulk powders, with particle sizes generally in the micrometer range. It has been shown that nanomaterials have properties that can enhance the performance of battery electrodes, owing to their superior electronic properties and surface areas. Some of the advantages of using nanostructured materials in battery electrodes include a higher electrode-electrolyte contact area, reduced mechanical strain from intercalation, and decreased electronic and ionic path lengths. ${ }^{45}$ Therefore, by applying the same concept to AIBs, significant performance enhancements should be expected. With this logic, it was decided to investigate graphite and vanadium oxide, two of the most promising cathode materials with an intercalation-based energy storage mechanism, and study the effect of nanostructure on their performance. The performance of various nanostructures has been studied as part of this $\mathrm{PhD}$ project: $\mathrm{V}_{2} \mathrm{O}_{5}$ nanofibres (Chapter 4), carbon nanofibres (Chapter 5), core-sheath nanofibres (Chapter 6), and a multi-layered nanostructured electrode (Chapter 7).

For the study of electrolytes, the research focus of this project is to find a low-cost alternative to the expensive organic chloride salts used in the current state-of-the art devices. Currently, [EMIm] $\mathrm{Cl}$ is arguably the most expensive component of AIBs (if the "standard" case of a graphite cathode is considered); therefore, finding an inexpensive alternative which could still work with good performance would significantly reduce the production costs of the technology. Eutectic mixtures of $\mathrm{AlCl}_{3}$ with acetamide and other simple amide analogues were studied as alternative electrolytes. This part of the $\mathrm{PhD}$ project is described in Chapter 8. 
Finally, Chapter 9 will briefly summarise the major findings outlined in this project and present the main goals towards which future research will be focused.

\section{Bibliography}

${ }^{1}$ C. Wolfram, O. Shelef, and P. Gertler, "How Will Energy Demand Develop in the Developing World?," Journal of Economic Perspectives, vol. 26, pp. 119-138, Feb. 2012.

${ }^{2}$ O. Morton, "Solar energy: A new day dawning?: Silicon Valley sunrise," Nature, vol. 443, pp. 1922, Sept. 2006.

${ }^{3}$ K. Branker, M. J. M. Pathak, and J. M. Pearce, "A review of solar photovoltaic levelized cost of electricity," Renewable and Sustainable Energy Reviews, vol. 15, pp. 4470-4482, Dec. 2011.

${ }^{4}$ M. A. Delucchi and M. Z. Jacobson, "Providing all global energy with wind, water, and solar power, Part II: Reliability, system and transmission costs, and policies," Energy Policy, vol. 39, pp. 1170-1190, Mar. 2011.

${ }^{5}$ H. Chen, T. N. Cong, W. Yang, C. Tan, Y. Li, and Y. Ding, "Progress in electrical energy storage system: A critical review," Progress in Natural Science, vol. 19, pp. 291-312, Mar. 2009.

${ }^{6}$ M. Ramsey, “24m Technologies Launches Cheaper-to-Produce Lithium-Ion Cell,” June 2015.

${ }^{7} \mathrm{~J}$. O. Besenhard and G. Eichinger, "High energy density lithium cells," Journal of Electroanalytical Chemistry and Interfacial Electrochemistry, vol. 68, pp. 1-18, Feb. 1976.

${ }^{8}$ K. Mizushima, P. C. Jones, P. J. Wiseman, and J. B. Goodenough, "LixCoO2 $(0<\mathrm{x}<-1)$ : A new cathode material for batteries of high energy density," Materials Research Bulletin, vol. 15, pp. 783789, June 1980.

${ }^{9}$ R. Yazami and P. Touzain, "A reversible graphite-lithium negative electrode for electrochemical generators," Journal of Power Sources, vol. 9, pp. 365-371, Jan. 1983.

${ }^{10}$ M. Armand and J.-M. Tarascon, "Building better batteries," Nature, vol. 451, pp. 652-657, Feb. 2008.

${ }^{11}$ R. Spotnitz and J. Franklin, "Abuse behavior of high-power, lithium-ion cells," Journal of Power Sources, vol. 113, pp. 81-100, Jan. 2003.

${ }^{12}$ D. P. Finegan, M. Scheel, J. B. Robinson, B. Tjaden, I. Hunt, T. J. Mason, J. Millichamp, M. Di Michiel, G. J. Offer, G. Hinds, D. J. Brett, and P. R. Shearing, "In-operando high-speed tomography of lithium-ion batteries during thermal runaway," Nature Communications, vol. 6, Apr. 2015.

${ }^{13}$ C. Hendricks, N. Williard, S. Mathew, and M. Pecht, "A failure modes, mechanisms, and effects analysis (FMMEA) of lithium-ion batteries," Journal of Power Sources, vol. 297, pp. 113-120, Nov. 2015.

${ }^{14}$ T. Waldmann, M. Wilka, M. Kasper, M. Fleischhammer, and M. Wohlfahrt-Mehrens, "Temperature dependent ageing mechanisms in Lithium-ion batteries - A Post-Mortem study," Journal of Power Sources, vol. 262, pp. 129-135, Sept. 2014.

${ }^{15}$ Q. Wang, P. Ping, X. Zhao, G. Chu, J. Sun, and C. Chen, "Thermal runaway caused fire and explosion of lithium ion battery," Journal of Power Sources, vol. 208, pp. 210-224, June 2012.

${ }^{16}$ F. S. Barnes and J. G. Levine, Large energy storage systems handbook. CRC press, 2011. 
${ }^{17}$ C. D. Parker, "Lead-acid battery energy-storage systems for electricity supply networks," Journal of Power Sources, vol. 100, pp. 18-28, Nov. 2001.

${ }^{18}$ Yung-Fang Yu Yao and J. T. Kummer, "Ion exchange properties of and rates of ionic diffusion in beta-alumina," Journal of Inorganic and Nuclear Chemistry, vol. 29, pp. 2453-2475, Sept. 1967.

${ }^{19} \mathrm{~J}$. Baker, "New technology and possible advances in energy storage," Energy Policy, vol. 36, pp. 4368-4373, Dec. 2008.

${ }^{20}$ I. Hadjipaschalis, A. Poullikkas, and V. Efthimiou, "Overview of current and future energy storage technologies for electric power applications," Renewable and Sustainable Energy Reviews, vol. 13, pp. 1513-1522, Aug. 2009.

${ }^{21}$ M. Rychcik and M. Skyllas-Kazacos, "Characteristics of a new all-vanadium redox flow battery," Journal of Power Sources, vol. 22, pp. 59-67, Jan. 1988.

${ }^{22}$ A. A. Yaroshevsky, "Abundances of chemical elements in the Earth's crust," Geochemistry International, vol. 44, pp. 48-55, Jan. 2006.

${ }^{23}$ V. Palomares, P. Serras, I. Villaluenga, K. B. Hueso, J. Carretero-González, and T. Rojo, "Na-ion batteries, recent advances and present challenges to become low cost energy storage systems," Energy E Environmental Science, vol. 5, no. 3, pp. 5884-5901, 2012.

${ }^{24}$ D. A. Stevens and J. R. Dahn, "High Capacity Anode Materials for Rechargeable Sodium-Ion Batteries," Journal of The Electrochemical Society, vol. 147, pp. 1271-1273, Jan. 2000.

${ }^{25}$ K. Zaghib, J. Trottier, P. Hovington, F. Brochu, A. Guerfi, A. Mauger, and C. M. Julien, "Characterization of Na-based phosphate as electrode materials for electrochemical cells," Journal of Power Sources, vol. 196, pp. 9612-9617, Nov. 2011.

${ }^{26}$ K. T. Lee, T. N. Ramesh, F. Nan, G. Botton, and L. F. Nazar, “Topochemical Synthesis of Sodium Metal Phosphate Olivines for Sodium-Ion Batteries," Chemistry of Materials, vol. 23, pp. 35933600, Aug. 2011.

${ }^{27}$ E. Levi, Y. Gofer, and D. Aurbach, "On the Way to Rechargeable Mg Batteries: The Challenge of New Cathode Materials," Chemistry of Materials, vol. 22, pp. 860-868, Feb. 2010.

${ }^{28}$ C. B. Bucur, T. Gregory, A. G. Oliver, and J. Muldoon, "Confession of a Magnesium Battery," The Journal of Physical Chemistry Letters, vol. 6, pp. 3578-3591, Sept. 2015.

${ }^{29}$ R. Mohtadi and F. Mizuno, "Magnesium batteries: Current state of the art, issues and future perspectives," Beilstein Journal of Nanotechnology, vol. 5, pp. 1291-1311, Aug. 2014.

${ }^{30}$ P. Saha, M. K. Datta, O. I. Velikokhatnyi, A. Manivannan, D. Alman, and P. N. Kumta, “Rechargeable magnesium battery: Current status and key challenges for the future," Progress in Materials Science, vol. 66, pp. 1-86, Oct. 2014.

${ }^{31}$ E. Lancry, E. Levi, Y. Gofer, M. Levi, G. Salitra, and D. Aurbach, "Leaching Chemistry and the Performance of the Mo6s8 Cathodes in Rechargeable Mg Batteries," Chemistry of Materials, vol. 16, pp. 2832-2838, July 2004.

${ }^{32}$ R. E. Doe, R. Han, J. Hwang, A. J. Gmitter, I. Shterenberg, H. D. Yoo, N. Pour, and D. Aurbach, "Novel, electrolyte solutions comprising fully inorganic salts with high anodic stability for rechargeable magnesium batteries," Chemical Communications, vol. 50, pp. 243-245, Nov. 2013.

${ }^{33}$ D. Gelman, B. Shvartsev, and Y. Ein-Eli, "Aluminum-air battery based on an ionic liquid electrolyte," Journal of Materials Chemistry A, vol. 2, no. 47, pp. 20237-20242, 2014.

${ }^{34}$ F. Xiang, X. Chen, J. Yu, W. Ma, Y. Li, and N. Yang, "Synthesis of three-dimensionally ordered porous perovskite type LaMnO3 for Al-air battery," Journal of Materials Science and Technology, vol. 34, no. 9, pp. 1532-1537, 2018. 
${ }^{35}$ N. Levy, M. Auinat, and Y. Ein-Eli, “Tetra-butyl ammonium fluoride - An advanced activator of aluminum surfaces in organic electrolytes for aluminum-air batteries," Energy Storage Materials, vol. 15, pp. 465-474, 2018.

${ }^{36}$ S. Liu, G. L. Pan, G. R. Li, and X. P. Gao, "Copper hexacyanoferrate nanoparticles as cathode material for aqueous Al-ion batteries," Journal of Materials Chemistry A, vol. 3, no. 3, pp. 959-962, 2015.

${ }^{37}$ H. Lahan, R. Boruah, A. Hazarika, and S. Das, "Anatase TiO2 as an Anode Material for Rechargeable Aqueous Aluminum-Ion Batteries: Remarkable Graphene Induced Aluminum Ion Storage Phenomenon," Journal of Physical Chemistry C, vol. 121, no. 47, pp. 26241-26249, 2017.

${ }^{38}$ A. Holland, R. Mckerracher, A. Cruden, and R. Wills, "An aluminium battery operating with an aqueous electrolyte," Journal of Applied Electrochemistry, vol. 48, no. 3, pp. 243-250, 2018.

${ }^{39}$ M. P. Paranthaman, G. Brown, X.-G. Sun, J. Nanda, A. Manthiram, and A. Manivannan, “A Transformational, High Energy Density, Secondary Aluminum Ion Battery," Meeting Abstracts, vol. MA2010-02, pp. 314-314, Aug. 2010.

${ }^{40}$ N. P. Stadie, S. Wang, K. V. Kravchyk, and M. V. Kovalenko, "Zeolite-Templated Carbon as an Ordered Microporous Electrode for Aluminum Batteries," ACS Nano, vol. 11, pp. 1911-1919, Feb. 2017.

${ }^{41}$ W. Wang, B. Jiang, W. Xiong, H. Sun, Z. Lin, L. Hu, J. Tu, J. Hou, H. Zhu, and S. Jiao, “A new cathode material for super-valent battery based on aluminium ion intercalation and deintercalation," Scientific Reports, vol. 3, Nov. 2013.

${ }^{42}$ M.-C. Lin, M. Gong, B. Lu, Y. Wu, D.-Y. Wang, M. Guan, M. Angell, C. Chen, J. Yang, B.-J. Hwang, and H. Dai, "An ultrafast rechargeable aluminium-ion battery," Nature, vol. 520, pp. 324-328, Apr. 2015.

${ }^{43}$ N. Jayaprakash, S. K. Das, and L. A. Archer, "The rechargeable aluminum-ion battery," Chemical Communications, vol. 47, no. 47, pp. 12610-12612, 2011.

${ }^{44}$ H. Chen, H. Xu, B. Zheng, S. Wang, T. Huang, F. Guo, W. Gao, and C. Gao, "Oxide Film Efficiently Suppresses Dendrite Growth in Aluminum-Ion Battery," ACS Applied Materials \& Interfaces, vol. 9, pp. 22628-22634, July 2017.

${ }^{45}$ A. S. Aricò, P. Bruce, B. Scrosati, J.-M. Tarascon, and W. van Schalkwijk, “Nanostructured materials for advanced energy conversion and storage devices," Nature Materials, vol. 4, pp. 366-377, May 2005. 
Chapter 2

\section{Literature review}





\subsection{Preface}

The following chapter provides a detailed overview of the literature on non-aqueous AIBs, from the initial discoveries leading to the development of the technology, until the most recent publications on cathode materials and electrolytes. A brief section outlining the major discoveries leading to the development of the lithiumion battery will also be presented hereafter.

\subsection{Background: Lithium-ion batteries}

The light weight and and low standard reduction potential $\left(-3.05 \mathrm{~V}\right.$ for the $\mathrm{Li}^{+} / \mathrm{Li}$ couple vs. standard hydrogen electrode) has always been a source of inspiration for its application in electrochemical energy storage. Some of the key pioneering works in this field include studies on the electroplating of metals in cyclic ester solvents such as ethylene carbonate and propylene carbonate, ${ }^{1}$ ionic conductivities in solids, ${ }^{2}$ and a theory for ion transfer in electrochemical cells. ${ }^{3}$

Initially, metallic lithium was considered to be the best option for the anodic material, and the main focus of research was therefore to find cathode materials which could allow intercalation of lithium ions at high transfer rates. One of the first successful results was a lithium-titanium disulfide $\left(\mathrm{Li}_{x} \mathrm{TiS}_{2}\right)$ cell, exhibiting a voltage of $2.5 \mathrm{~V}$, as reported by Whittingham and coworkers. ${ }^{4,5}$ The fundamental design flaw of this cell, however, was the formation of dendrites on the anode surface, causing the piercing of the separator and eventually leading to short circuits after repeated cycling.

The dendrite issue drove researchers to investigate alternative, intercalation-based anode materials, which could lead to safer battery architectures. This would, however, have a negative impact on the cell potential, as the intercalation reaction into anodic materials would inevitably happen at less negative potentials compared to $\mathrm{Li}$ electroplating. Graphite was initially proposed as a candidate, ${ }^{6}$ but issues with its exfoliation and destruction upon intercalation hindered any further development.

Contemporary to anode development, new cathode materials were being studied. A significant breakthrough was the discovery of the properties of lithium cobalt 
oxide $\left(\mathrm{Li}_{\mathrm{x}} \mathrm{CoO}_{2}\right)$ as a cathode by Goodenough's research group. ${ }^{7}$ The material showed a high redox potential, and fast ion diffusion kinetics.

The discovery of high-voltage cathodes further promoted the search for carbonaceous anode materials. Petroleum coke was found by Yoshino and coworkers ${ }^{8}$ to be particularly effective, thanks to its presence of both crystalline and amorphous domains, allowing for reversible intercalation of a large number of lithium ions without significant exfoliation. Ultimately, thanks to the discovery of solidelectrolyte interphase (SEI)-forming electrolytes, ${ }^{9,} 10$ graphite was also found to be a viable and high-energy density anode material: the formation of a film on the surface of the material by the decomposition of electrolyte species, protects the anode from exfoliation and disintegration.

In summary, the key findings outlined in this section, together with the discoveries of additional cathode materials such as $\mathrm{Li}_{x} \mathrm{Mn}_{3} \mathrm{O}_{4}{ }^{11}$ and $\mathrm{LiFePO}_{4}{ }^{12}$ led to the development of the lithium-ion battery as we know it. Thanks to the profound impact that this technology had on the world, The 2019 Nobel prize in Chemistry was awarded to Goodenough, Whittingham and Yoshino.

\subsection{History of the aluminium-ion battery}

Some of the earliest attempts to use aluminium as a battery electrode trace back to the 1850s: ${ }^{13}$ several cell architectures had been proposed, implementing pure or amalgamated aluminium either as anodes or cathodes. Another early example of the use of aluminium anodes can be found from a 1953 patent, describing the development of a primary $\mathrm{Al} / \mathrm{MnO}_{2}$ battery, using manganese chloride tetrahydrate as electrolyte. ${ }^{14}$ Studies on the aluminium/oxygen system are also present, which were first described by Zaromb ${ }^{15}$ and Bockstie et al. ${ }^{16}$ These first efforts, however, were generally not very successful due to the inability to overcome the passivating oxide layer forming on the electrode surface. ${ }^{17,18}$

The origins of the current cell design are arguably imputable to the discovery of the ability of aluminium trichloride to form eutectic mixtures, with melting points

below room temperature when mixed with certain organic chloride salts. ${ }^{19-21}$ More importantly, it was found that if $\mathrm{AlCl}_{3}$ is present in a molar fraction higher than 0.5 , the resulting mixture possesses Lewis acid characteristics and can reversibly 
electrodeposit aluminium metal onto metallic surfaces. This is thanks to the presence of the $\mathrm{Al}_{2} \mathrm{Cl}_{7}^{-}$ion, which allows the mixtures to be used as an electrolyte system for primary or secondary batteries. ${ }^{22,23}$ The first cells proposed using organic chloride salts and $\mathrm{AlCl}_{3}$ as electrolytes were based on systems such as $\mathrm{Al}-\mathrm{FeS}_{2}$, $\mathrm{Al}-\mathrm{CuCl}_{2}, \mathrm{Al}-\mathrm{Cl}_{2},{ }^{24}$ and $\mathrm{Al}-\mathrm{FeCl}_{3} .{ }^{25}$

A promising attempt was made by Paranthaman et al. in 2010, who proposed a cell using metallic $\mathrm{Al}$ as anode, $\mathrm{AlCl}_{3} /[\mathrm{EMIm}] \mathrm{Cl}$ as electrolyte, and spinel-phase $\lambda-\mathrm{Mn}_{2} \mathrm{O}_{4}$ as cathode (Figure 2.1). ${ }^{26}$ The authors argued that the presence of $\mathrm{AlCl}_{4}^{-}$species in the electrolyte prevented the formation of an oxide layer in the aluminium electrode surface and suppressed hydrogen evolution. Furthermore, the authors also claimed that the complexation of aluminium to form $\mathrm{Al}_{2} \mathrm{Cl}_{7}^{-}$had the beneficial effect of inhibiting dendrite formation. How-

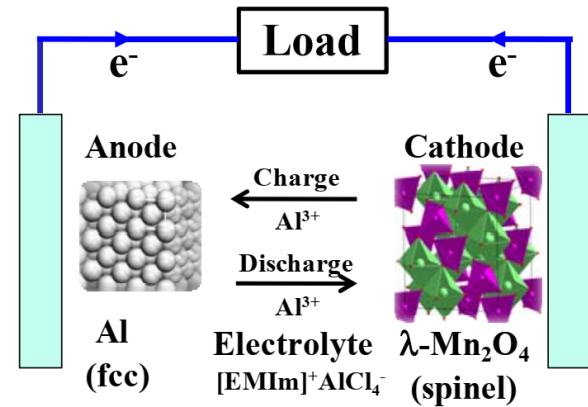

FIGURE 2.1: Schematic representation of the AIB prototype proposed by Paranthaman et al. (republished with permission from the publication). ${ }^{26}$

ever, not much evidence behind these claims was provided. Cyclic voltammetry experiments were presented to prove the deposition and dissolution of $\mathrm{Al}$ but unfortunately, no intercalation capacity was achieved by the $\lambda-\mathrm{Mn}_{2} \mathrm{O}_{4}$ cathode. Nevertheless, this work inspired a great deal of interest in the topic and new research was published shortly after describing working prototypes of rechargeable Al-ion cells. The following year, Jayaprakash et al. ${ }^{27}$ finally proposed the first working rechargeable aluminium- $-\mathrm{V}_{2} \mathrm{O}_{5}$ battery, which showed stable, reversible electrochemistry, and a remarkable discharge capacity of $305 \mathrm{mAh} \mathrm{g}^{-1}$ for the first discharge cycle. Although the authors only reported activity for the first 20 cycles of the cell which displayed poor coulombic efficiencies, this was an important breakthrough, as it finally showed the concrete possibility of building stable secondary batteries using the $\mathrm{Al} / \mathrm{AlCl}_{3}$ system. 


\subsection{Cathode materials}

\subsubsection{Vanadium oxide}

The aforementioned first report of a working AIB by Jayaprakash et al. used $\mathrm{V}_{2} \mathrm{O}_{5}$ as the cathodic material. ${ }^{27}$ The battery displayed an open circuit voltage of 1.8 $\mathrm{V}$ and a discharge curve showing a long plateau at about $0.55 \mathrm{~V}$, suggesting the insertion of $\mathrm{Al}^{3+}$ ions into the cathode. The authors calculated that the theoretical capacity of $\mathrm{V}_{2} \mathrm{O}_{5}$ would be $442 \mathrm{mAh} \mathrm{g}^{-1}$. This was based on the assumption that the electrochemical reaction taking place at the cathode would be the conversion of $\mathrm{V}_{2} \mathrm{O}_{5}$ into $\mathrm{AlV}_{2} \mathrm{O}_{5}$, according to the following mechanism:

$$
\begin{aligned}
& \text { Cathode: } \quad 4 \mathrm{Al}_{2} \mathrm{Cl}_{7}^{-}+\mathrm{V}_{2} \mathrm{O}_{5}+3 \mathrm{e}^{-} \rightleftharpoons \mathrm{Al}\left(\mathrm{V}_{2} \mathrm{O}_{5}\right)+7 \mathrm{AlCl}_{4}^{-} \\
& \text {Anode: } \\
& \mathrm{Al}+7 \mathrm{AlCl}_{4}^{-} \rightleftharpoons 4 \mathrm{Al}_{2} \mathrm{Cl}_{7}^{-}+3 \mathrm{e}^{-}
\end{aligned}
$$

However, no experimental data was presented to support this claim. In response to this work, Reed and Menke ${ }^{28}$ published new work examining the role of the $\mathrm{V}_{2} \mathrm{O}_{5}$ cathode. The authors fabricated a $\mathrm{V}_{2} \mathrm{O}_{5}$ aerogel using a well-known literature procedure, ${ }^{29}$ and used it as the active cathode material in prototype devices. Cyclic voltammetry data indicated that when a platinum current collector was used, no peaks were observed in the $0-1.5 \mathrm{~V}$ window. Instead, when a stainless steel current collector was used, a series of redox peaks were observed.

These findings led the authors to believe that any electrochemical activity in the device claimed by the previous paper by Jayaprakash et al. must be attributed exclusively to the reaction of iron and chromium metals in the current collector with the electrolyte, and $\mathrm{V}_{2} \mathrm{O}_{5}$ has no actual role in the cell electrochemistry. In support to their claim, the authors also provided scanning electron microscopy (SEM) images of the separators used in the prototype cells after a series of charge-discharge cycles. The images showed the presence of dendrites, which were revealed to contain $\mathrm{Fe}, \mathrm{Cr}, \mathrm{Al}, \mathrm{Si}$, and $\mathrm{Cl}$ from energy dispersive $\mathrm{X}$-ray (EDX) spectroscopy elemental analysis.

The findings of Reed and Menke's publication therefore appeared to put some serious limitations on the feasibility of $\mathrm{V}_{2} \mathrm{O}_{5}$ as a cathode material as well as the 
use of stainless steel as a current collector. However, despite the issues raised by this publication, another important work was released by Wang et al. ${ }^{30}$ in late 2014. In this paper, a new cathode was proposed, which was fabricated by the hydrothermal growth of $\mathrm{V}_{2} \mathrm{O}_{5}$ on a nickel foam substrate. Besides showing an improvement in specific capacity, the authors also performed a control test, using a cathode containing no vanadium oxide. The cell yielded no discharging specific capacity whatsoever, suggesting that $\mathrm{V}_{2} \mathrm{O}_{5}$ indeed plays a fundamental role in the battery, and thus disproving the arguments sustained by Reed and Menke. ${ }^{28}$ More publications refuting the claims of the inactivity of $\mathrm{V}_{2} \mathrm{O}_{5}$ in AIBs were later released. First, Chiku et al. ${ }^{31}$ demonstrated the possibility of $\mathrm{Al}^{3+}$ intercalating into amorphous $\mathrm{V}_{2} \mathrm{O}_{5}$. This was supported by $\mathrm{X}$-ray photoelectron spectroscopy (XPS) data, suggesting a change in the oxidation state of vanadium ion in charged and discharged $\mathrm{V}_{2} \mathrm{O}_{5}$ electrodes. A molybdenum current collector was used to avoid any potential side reactions caused by of stainless steel. CV and EDX data was also provided, demonstrating the electrochemical stability of Mo in the device.

In a later publication, $\mathrm{Gu}$ et al. ${ }^{32}$ provided evidence for the intercalation of $\mathrm{Al}^{3+}$ in $\mathrm{V}_{2} \mathrm{O}_{5}$ nanowires. This study presented high resolution TEM images (Figure 2.2), showing the disruption of the crystal structure and formation of an amorphous layer on the outer lattice layers of the $\mathrm{V}_{2} \mathrm{O}_{5}$ nanostructures after the first discharge sequence. Furthermore, an ion storage mechanism involving both intercalation and phase-transition reactions was proposed. Therefore, although the effectiveness of $\mathrm{V}_{2} \mathrm{O}_{5}$ as a cathode material initially appeared to be a debated subject, with different research groups presenting conflicting results, the evidence provided by the latest publications strongly confirms that the $\mathrm{Al}-\mathrm{V}_{2} \mathrm{O}_{5}$ battery is indeed a viable system, showing good potential and room for improvement.

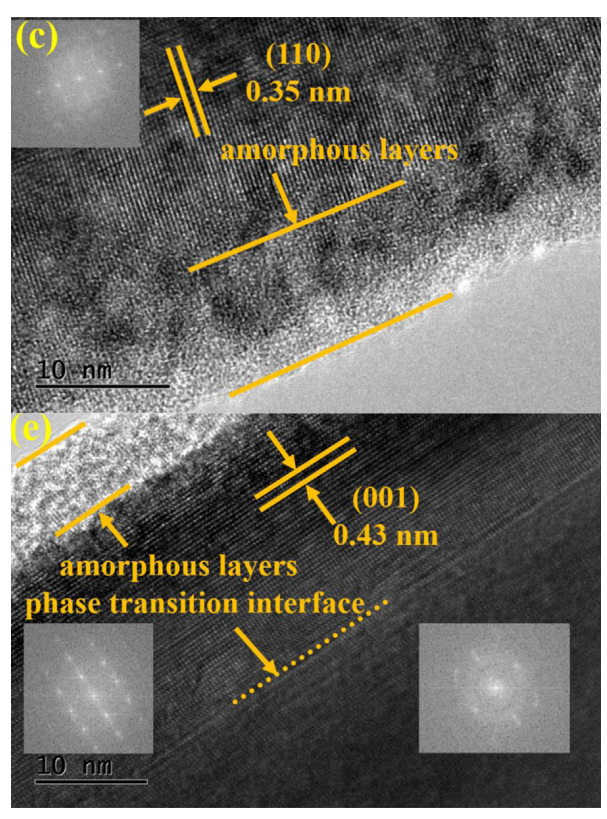

FIGURE 2.2: TEM images showing the formation of amorphous $\mathrm{V}_{2} \mathrm{O}_{5}$ after the intercalation of $\mathrm{Al}^{3+}$ ions (Adapted with permission from the publication by Gu et al..). ${ }^{32}$ 
$\mathrm{V}_{2} \mathrm{O}_{5}$ is not the only vanadium compound known to show activity in AIBs, as vanadium (IV) oxide $\left(\mathrm{VO}_{2}\right)$ was also reported to function as a cathode material. A paper published by Wang et al..$^{33}$ in 2013 argued that the monoclinic crystal structure of $\mathrm{VO}_{2}$ presents large channels which could allow the intercalation of $\mathrm{Al}^{3+}$ ions. The publication reports basic electrochemical testing, claiming a discharge capacity of about $165 \mathrm{mAh} \mathrm{g}^{-1}$, with a voltage plateau of $0.5-0.6 \mathrm{~V}$. The authors also reported a remarkable cycle life of over 100 cycles, albeit correlated with a significant drop in specific capacity.

\subsubsection{Carbon}

Carbon is easily the most studied candidate for a cathode material in these systems. One of the first attempts to implement graphitic materials in $\mathrm{Al}$ batteries was made by Rani et al. ${ }^{34}$ They proposed a cathode based on fluorinated natural graphite, which was inspired from the studies of graphite fluorides in Liion batteries ${ }^{35}$ and early prototypes of non-aqueous Al batteries. ${ }^{36}$ The cells displayed remarkable specific capacity and stability over 20 cycles, but poor coulombic efficiency. Shortly after, Sun et al. reported an Al-ion battery fabricated using graphitic carbon paper as the cathode. ${ }^{37}$ The cell exhibited a $1.8 \mathrm{~V}$ plateau during discharge, and great reversibility at cycling rates up to $100 \mathrm{~mA} \mathrm{~g}^{-1}$.

Perhaps one of the most important papers in the entire AIB literature is the work published by Lin et al. in 2015. ${ }^{38}$ The authors presented an "ultrafast rechargeable aluminium-ion battery", using a "three-dimensional graphitic foam cathode". The battery could withstand cycling rates up to $5000 \mathrm{~mA} \mathrm{~g}^{-1}$ without any significant loss in capacity, while at the same time maintaining $>95 \%$ coulombic efficiency. The cathode was fabricated by growing graphitic carbon on a commercial nickel foam substrate using chemical vapour deposition (CVD) techniques. The battery also showed well-defined voltage plateaus around $2 \mathrm{~V}$, and exhibited excellent stability over 7500 cycles. The main drawbacks of the device consisted in a slightly lower coulombic efficiency at the slowest charging speeds reported $\left(100 \mathrm{~mA} \mathrm{~g}^{-1}\right)$,

and an overall sub-par specific discharge capacity of around $66 \mathrm{mAh} \mathrm{g}^{-1}$. Even so, the resulting energy density for the devices amounted to about $40 \mathrm{Wh} \mathrm{kg}^{-1}$, a value comparable to lead-acid and Ni-MH batteries, and the achievable power 
density is up to $3,000 \mathrm{~W} \mathrm{~kg}^{-1}$, which is in the range of supercapacitors. The authors also argued that the species intercalating into the cathode were in fact the negatively charged chloroaluminate ions, $\mathrm{AlCl}_{4}^{-}$, which are naturally present in eutectic mixtures of $\mathrm{AlCl}_{3}$ and organic chloride salts, as suggested by earlier studies. ${ }^{39,40}$ Therefore, according to this model, the intercalation event would have to take place during the charge phase, and deintercalation would happen as the battery gets discharged. The chemistry of these events can be described according to the following reaction:

$$
\mathrm{xC}+\mathrm{AlCl}_{4}^{-} \rightleftharpoons \mathrm{C}_{\mathrm{x}}\left(\mathrm{AlCl}_{4}\right)+\mathrm{e}^{-}
$$

This claim was supported by XRD, Raman spectroscopy, XPS and Auger electron spectroscopy. This mechanism is notably different to other "ion" battery chemistries in which positively charged ions are inserted into the cathode during the discharge phase.

The impressive features claimed by the work of Lin et al. were soon followed by a series of works aimed to improve on the same concept. One interesting variation was proposed by Yang et al., ${ }^{41}$ who attempted to address the issue of low specific capacity reported in the aforementioned paper. The authors fabricated a 3D graphene mesh network by a chemical vapour deposition (CVD) process, using folded Ni meshes as support, which were then chemically etched to yield a free-standing graphene-like structure. The resulting material, which displayed remarkable mechanical and electronic properties on its own, produced comparable electrochemical performance to the material synthesised by Lin et al. when used in an Al-ion cell. No sensible improvements, other than the novel fabrication method for the cathode material, were therefore introduced by this work. A similar method was then adopted by the same research group responsible for the ultrafast AIB paper ${ }^{38}$ to produce a free-standing graphene foam, which was additionally treated with an $\mathrm{Ar}^{+}$plasma to etch the surface and produce cavities and ribbon-like features on the surface of the graphene sheets. This was believed to further increase the surface area of the electrode, and create additional "entry points" for the insertion of chloroaluminate ions. When the material was implemented in a device, the same outstanding ultrafast cycling capabilities and cycle life were obtained, but the specific capacity was significantly increased (up to 148 $\mathrm{mAh}^{-1}$ ). The cell also was also shown to function with remarkable stability over 
a broad range of temperatures, from 0 to $80^{\circ} \mathrm{C} .{ }^{42}$ A different approach was proposed by Stadie et al., ${ }^{43}$ who used zeolite-templated carbon as a cathode in their cell. The reasoning behind this choice is that the authors disagreed with the claims of the aforementioned reports, as they believed that the size of the chloroaluminate ions would be too large to allow their insertion into a graphite lattice; therefore, any electrochemical activity in a graphite or graphenebased cathode must be exclusively imputable to a capacitive or pseudo-capacitive surface process. Consequently, a carbonaceous material with a high surface area would yield very high specific capacities. Zeolite-templated car-

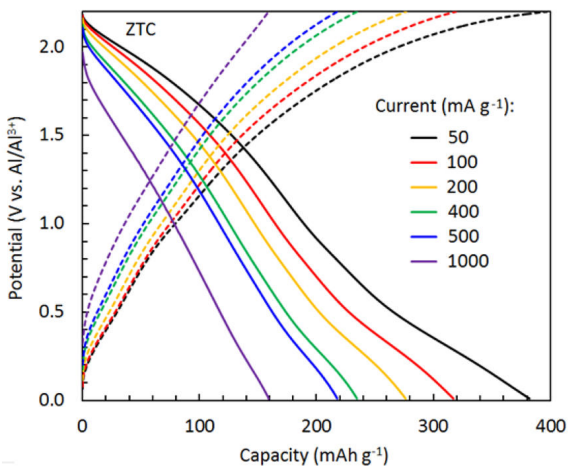

FIGURE 2.3: Galvanostatic cycling profiles of AIB devices using zeolite-templated carbon as cathode (Adapted with permission from the publication by Stadie et al.). ${ }^{43}$ bon has one of the highest specific surface areas of all known materials, and therefore would be an ideal candidate for this cell architecture. The device built using this material indeed showed an unprecedented specific capacity for an aluminium-based battery (up to $380 \mathrm{mAh} \mathrm{g}^{-1}$ ), but had no well-defined voltage plateau over its charging and discharging curves (Figure 2.3*); this type of behaviour is typical of capacitive (surface adsorption-based) processes, and closely resembles the one observed for electrochemical capacitors. The capacity also decreased sensibly at higher cycling rates, much more drastically than any other work utilising carbon cathodes. The results achieved by this paper would thus appear in evident contrast with the works of other authors, who had so far claimed that the battery relied on an intercalation mechanism at the cathode.

Although the excellent performance of CVD-prepared carbon materials makes them an exciting research topic, their fabrication method does not suit well the requirements for mass production and commercialisation. Because of this, a series of papers presenting low-cost alternative carbon materials for AIBs have been published. ${ }^{44-47}$ One of these works was published by Wang et al., ${ }^{45}$ who presented an AIB cell using pristine natural graphite flakes as cathode. The cell exhibited

*Source: https://pubs.acs.org/doi/10.1021/acsnano.6b07995 - further permissions related to this Figure should be directed to the American Chemical Society. 
good performance, despite the relatively simple composition of the electrode. An interesting insight uncovered by this work is that the structural modifications of the graphite caused by intercalation and deintercalation of $\mathrm{AlCl}_{4}^{-}$appear perfectly reversible by XRD and Raman spectroscopy. Conversely, when the same study was conducted on the "graphitic foam", first described by Lin et al. ${ }^{38}$ the results showed that the graphitic foam underwent an irreversible structural transformation after the first charge-discharge cycle. More recently, a publication by Elia et al. also showed evidence of the partial irreversiblility of the process, which was attributed to a partial trapping of $\mathrm{AlCl}_{4}^{-}$ions within the layers of the pyrolytic graphite paper cathode used, and a consequential structural reorganisation of the material. ${ }^{48}$ Other reports also observed this phenomenon on graphite-based cathodes, but attribute the behaviour to the formation of a solid-electrolyte interphase (SEI), but not much experimental evidence is provided. ${ }^{49,50}$

Similar results were achieved by Kravchyk et al., ${ }^{46}$ who used natural graphite flakes in their cell to produce a device with remarkable performance while reducing the overall production costs. This work also provides some additional evidence of the intercalation of $\mathrm{AlCl}_{4}^{-}$ions via solution- and solid-state ${ }^{27} \mathrm{Al}$ nuclear magnetic resonance (NMR) spectroscopy. Most importantly, this paper raises two very critical issues: since there is no unidirectional flow of any of the Al-containing species in the cell, the battery does not formally involve a rocking-chair mechanism such as the one characteristic of LIBs. Instead, the authors refer to it as a "mixed-ion operation", as the ionic species involved in the anode are different from the ones involved in the cathode. The two semi-reactions taking place during the charging process are the following:

Cathode:

Anode:

$$
\mathrm{xC}+\mathrm{AlCl}_{4}^{-} \rightleftharpoons \mathrm{C}_{\mathrm{x}}\left(\mathrm{AlCl}_{4}\right)+\mathrm{e}^{-}
$$

$$
4 \mathrm{Al}_{2} \mathrm{Cl}_{7}^{-}+3 \mathrm{e}^{-} \rightleftharpoons 7 \mathrm{AlCl}_{4}^{-}+\mathrm{Al}
$$

It is well documented that the $\mathrm{Al}_{2} \mathrm{Cl}_{7}^{-}$ion is essential to the reversible electroplating of $\mathrm{Al}$ at the anode. ${ }^{23,51,52}$ This also implies that, according to Equation 2.3b, the charging process of an Al-graphite battery must be complete when the electrolyte is depleted of all $\mathrm{Al}_{2} \mathrm{Cl}_{7}^{-}$ions. Because the concentration of $\mathrm{Al}_{2} \mathrm{Cl}_{7}^{-}$in the electrolyte mixture is proportional to the quantity of $\mathrm{AlCl}_{3}$ added, ${ }^{53}$ the authors 
stated that $\mathrm{AlCl}_{3}$ must have a limiting effect on the energy density of the cell. Because of this, the actual anode material of the battery would not be aluminium metal, but rather the dissolved $\mathrm{AlCl}_{3}$ in the electrolyte mixture. The $\mathrm{Al}$ metal electrode would therefore technically not be required for the battery to function, as any conductive substrate allowing the electrodeposition of $\mathrm{Al}$ could be used. Based on this assumption, Kravchyk et al. argue that the concentration of $\mathrm{AlCl}_{3}$ in the electrolyte mixture must be maximised in order to achieve the best energetic density possible. This value was found to be around the 2:1 molar ratio for the $\mathrm{AlCl}_{3} /[\mathrm{EMIm}] \mathrm{Cl}$ eutectic. Using this "saturated" electrolyte mixture, the authors obtained a device which, despite having a lower specific capacity for the cathode, exhibited an enhanced overall specific energy of $62 \mathrm{Wh} \mathrm{kg}^{-1}$. This value is exciting for commercialising AIBs as it is comparable with current technologies such as redox-flow batteries and lead-acid batteries. For comparison, the calculated specific energy value for the device obtained with a 1.3:1 ratio electrolyte was calculated to be $33 \mathrm{Wh} \mathrm{kg}^{-1}$ (Figure $2.4^{\dagger}$ ).

The mechanism of intercalation $\mathrm{AlCl}_{4}^{-}$has also been studied in great detail. A few theoretical studies have been conducted and one of the first works on the subject is the publication by Bhauriyal et al., who conducted firstprinciples calculations on the staging mechanism of the chloroaluminate ion in the graphite lattice. This study indicates that $\mathrm{AlCl}_{4}^{-}$can in fact intercalate into graphite during the charging process, likely causing the oxidation of the carbon material. The results also hint at the possible driving forces for the "ultrafast" cycling capabilities of graphitic cathodes. More

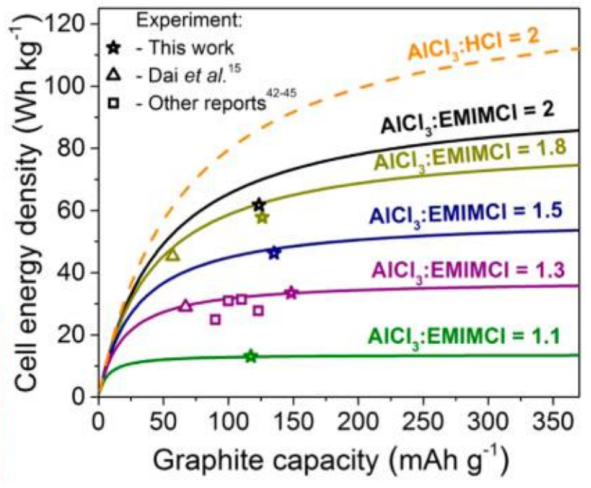

FIGURE 2.4: Energy densities as a function of the $\mathrm{AlCl}_{3} /[\mathrm{EMIm}] \mathrm{Cl}$ molar ratio, as proposed by Kravchyk et al. (Adapted with permission from the publication). ${ }^{46}$ theoretical studies are reported in the paper by Wang et al., ${ }^{45}$ which support the work by Bhauriyal et al., and suggest that the tetrahedral geometry of $\mathrm{AlCl}_{4}^{-}$could be distorted when the ion is intercalated. Another study by Wu et al. argues that $\mathrm{AlCl}_{4}^{-}$might even completely flatten into a square planar structure, which would

\footnotetext{
${ }^{\dagger}$ Source: https://pubs.acs.org/doi/10.1021/acs.chemmater.7b01060 - further permissions related to this Figure should be directed to the American Chemical Society.
} 
allow its fast diffusion into the graphite lattice. ${ }^{54}$ More recently, a few in-depth experimental studies on the subject have also been performed. Greco et al. ${ }^{55}$ investigated a pyrolytic graphite cathode by $\mathrm{XRD}$, small angle X-ray spectroscopy (SAXS), and tomography analysis. It was found that a stage 4 graphite intercalation compound ${ }^{\ddagger}$ is formed when the cathode is fully charged. This is in disagreement with previous works, who claimed that stage 1 could be achieved at full charge. ${ }^{57,58}$ The authors also argued that the intercalation process is not fully reversible, as the cathode can only revert back to stage 6 after the first discharge. This phenomenon, combined with the loss of porosity in the electrode, could be yet another cause for the low coulombic efficiency in the first charge-discharge cycle, reported in a few publications for graphitic materials. ${ }^{45,48-50}$ An in situ XRD study by Pan et al. also claims that stage 4 corresponds to a fully charged graphite cathode. ${ }^{59}$ In addition, the authors found evidence that if the device is charged at low temperatures $\left(-10^{\circ} \mathrm{C}\right)$, then stage 3 intercalation can also be achieved, leading to an increase in the maximum achievable specific capacity and energy density of the cathode.

The publications described in this review therefore show that graphitic materials are indeed an attractive candidate for AIB cathodes. Plenty of examples of devices can be built using inexpensive and abundant materials, and those showing performance on par with current commercial technologies can be found.

\subsubsection{Other materials}

Although carbon and vanadium oxide are two of the most studied materials for AIB cathodes, many other materials have been studied for the same purpose. Generally speaking, the performance of such materials can be quite variable, but further investigation could nonetheless lead to interesting discoveries and better performing devices.

\section{Polymers and small molecules}

An early publication by Hudak explored the possibility of using conductive polymers as cathode materials in AIBs. ${ }^{60}$ The paper describes an elegant method for

\footnotetext{
${ }^{\ddagger}$ A stage $n$ intercalation compound indicates a material in which a layer of intercalating species is present between $n$ lattice planes (where $n$ is a positive integer). ${ }^{56}$
} 
the preparation of chloroaluminate-doped polypyrrole or polythiophene films by electropolymerisation of monomer solutions directly in the $\mathrm{AlCl}_{3} /[\mathrm{EMIm}] \mathrm{Cl}$ electrolyte. The polymer films showed promising results: specific capacities up to $85 \mathrm{mAh} \mathrm{g}^{-1}$ over a $0.8-2 \mathrm{~V}$ range were reported, with good coulombic efficiency $(>91 \%)$, and a capacity loss of about $18 \%$ after 100 cycles. Similar works were also published later by Schoetz et al., who proposed a cathode based on poly-(3,4ethylenedioxythiophene), and Walter et al., who proposed polypyrenes as high performance cathode materials. ${ }^{61}$ More recently, Fan et al. proposed a "universal organic cathode" based on a carbon nanotube-polyimide composite. Interestingly, this material allows fast charging rates and high capacities not only for AIBs, but also Li-ion and Mg-ion batteries. ${ }^{62}$

An interesting recent development was presented by Kim et al., who developed a redox-active macrocyclic organic compound, which was used as a cathode material for AIBs. ${ }^{63}$ According to the authors, the phenanthrenequinone-based molecule is able to intercalate and form a complex with $\mathrm{AlCl}_{2}^{+}$ions, as suggested by ex situ time-of-flight secondary ion mass spectrometry (TOF-SIMS) studies. This is an important discovery, as it could potentially constitute a significant step forward in the increasing of the energy density of the battery. A mechanism involving $\mathrm{AlCl}_{2}^{+}$ions instead of $\mathrm{AlCl}_{4}^{-}$would correspond in a more efficient utilisation of the electrolyte, and the device would therefore require a lower quantity of it to function. Furthermore, this result also gives hope towards the realisation of a true $\mathrm{Al}^{3+}$ storage mechanism in organic cathodes, which would correspond to the most efficient mechanism in terms of electrolyte utilisation. ${ }^{64}$

\section{Sulphur, selenium, and metal chalcogenides}

Metal chalcogenides have recently attracted some attention in AIB research as potential cathodes. The first material belonging to this family which was proposed is $\mathrm{Mo}_{6} \mathrm{~S}_{6}$ : although the material is mostly known for its use in $\mathrm{Mg}$ ion batteries, ${ }^{65}$ a recent publication revealed the possibility of its use as a cathode for AIBs. ${ }^{66}$ The paper provided evidence of the intercalation of $\mathrm{Al}^{3+}$ ions in the form of both crystallographic and electrochemical data. A maximum experimental capacity of 167 $\mathrm{mAh} \mathrm{g}^{-1}$, in somewhat good agreement with the theoretical model proposed by the authors, was reported. The material, however, showed a few critical issues: capacity appears to fall drastically after the first 2-3 cycles, and coulombic efficiency 
is greater than $100 \%$ (i.e. the discharge capacity is higher than the charge capacity). The authors attributed this to trapped $\mathrm{Al}$ ions in the crystal lattice, leading to an incomplete charging step. A recent report by Li et al. ${ }^{67}$ shows that $\mathrm{MoS}_{2}$ can also work as a cathode for AIBs. Similar issues were also encountered in the material, which showed poor reversibility. The authors ascribed the problem to a phase change in the material, and the formation of an SEI. Furthermore, the energy storage mechanism was found to be mainly capacitive or pseudo-capacitive, as suggested by the lack of faradaic peaks in the cyclic voltammograms. Somewhat similar behaviours were also reported by several works for other metal chalcogenides such as $\mathrm{VS}_{2}{ }^{68} \mathrm{Co}_{3} \mathrm{~S}_{4},{ }^{69}$ and CoSe. ${ }^{70}$ A more satisfying result was obtained by $\mathrm{Hu}$ et al., ${ }^{71}$ who fabricated a carbon nanofibre-carbon nanotube- $\mathrm{Co}_{9} \mathrm{~S}_{8}$ composite to be used as an AIB cathode. The material displayed a well-defined galvanostatic profile, with a stable discharge plateau at about $0.8 \mathrm{~V}$. Furthermore, remarkable discharge capacities and cycle lives were observed in the tests. In another report, Liang et al. also obtained good results from a nanostructured SnS film. ${ }^{72}$ Interestingly, the authors claimed that the energy storage mechanism is based on the intercalation of $\mathrm{AlCl}_{4}^{-}$ions at the cathode during the charging phase, in a similar fashion to graphite materials. Non-stochiometric $\mathrm{Cu}_{2-x} \mathrm{Se}$ was also recently proposed as a cathode material, showing similar performance. ${ }^{73}$

A significantly different cathode material is elemental sulphur. Inspired by Li-S and Na-S technologies, Cohn et al. ${ }^{74}$ proposed an Al-S cell using [EMIm] $\mathrm{Cl} / \mathrm{AlCl}_{3}$ as electrolyte (Figure 2.5). The assumed redox mechanism is not based on intercalation, but rather on a conversion reaction.The authors reported an outstanding discharge capacity of over $1400 \mathrm{mAh} \mathrm{g}^{-1}$, approaching the theoretical capacity of sulphur (1672 $\mathrm{mAh} \mathrm{g}^{-1}$ ) with a long plateau at about $1.2 \mathrm{~V}$ for the first discharge cycle. However, during subsequent cycles,

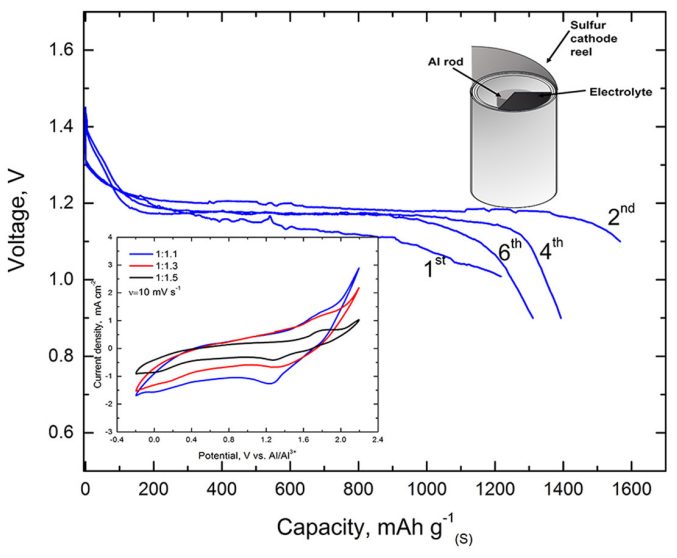

FIGURE 2.5: Graphical abstract of the publication by Cohn et al, presenting a rechargeable Al-S battery (Adapted with permission from the publication). ${ }^{74}$ the capacity immediately plummeted to much lower values, with the voltage steadily declining. This was attributed to the dissolution of sulphides produced 
by the discharging reaction in the electrolyte. This was supported by XPS, SEM and UV-visible absorption data. The conversion mechanism taking place at the cathode can be described by the following reaction equation:

$$
2 \mathrm{Al}^{3+}+3 \mathrm{~S}+6 \mathrm{e}^{-} \rightleftharpoons \mathrm{Al}_{2} \mathrm{~S}_{3}
$$

A similar behaviour was observed using pyrite-phase $\mathrm{FeS}_{2}$ as cathode material, as described by Mori et al. ${ }^{75}$ The authors presented a device employing a pyrite cathode, working on a similar reversible conversion mechanism as the work discussed above. The battery described in this publication, operating at $55{ }^{\circ} \mathrm{C}$, exhibited a rather large discharge capacity during the first cycle (ca. $600 \mathrm{mAh} \mathrm{g}^{-1}$ ), but poor cycle stability due to the dissolution of the aluminium sulphide species. A recent effort to reduce the issue of polysulphide dispersion was made by $\mathrm{Yu}$ and Manthiram, ${ }^{76}$ where the authors showed that the capacity retention could be improved significantly by using a carbon nanotube-coated separator and using carbon nanofibres as a scaffold to support elemental sulphur.

Elemental selenium was also shown to function as a cathode for AIBs by Huang et al. ${ }^{77}$ In this case, a conversion mechanism involving the formation of $\mathrm{Se}_{2} \mathrm{Cl}_{2}$ is involved. This compound is a room-temperature liquid which could also diffuse in the electrolyte, leading to a specific capacity loss. However, the authors claim that the addition of mesoporous carbon in the cathode can counteract this effect by effectively adsorbing the generated $\mathrm{Se}_{2} \mathrm{Cl}_{2}$ and serving as a reservoir for the process. Compared to sulphur, this material can provide a more modest specific capacity $\left(178 \mathrm{mAh} \mathrm{g}^{-1}\right)$, but a longer and notably more stable cycling life.

\section{Other cathode materials}

Materials with a two-dimensional or layered crystal structure are often an obvious choice for ion intercalation-based energy storage mechanism. VahidMohammadi et al..$^{78}$ investigated the use of vanadium carbide, a material belonging to the family of MXenes, $\$$ as a cathode material for AIBs. The authors claimed that, unlike what was reported for other applications, the material showed well-defined faradaic behaviour, as demonstrated by the presence of clear discharge plateaus

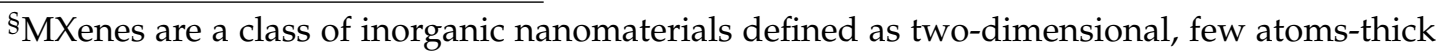
transition metal carbides, nitrides, or carbonitrides. ${ }^{79}$
} 
at about $1 \mathrm{~V}$ in the discharge profile. This process was ascribed to the reversible intercalation of $\mathrm{Al}^{3+}$ ions.

The presence of large channels in the crystal structure of materials is also a great incentive to investigate their properties as intercalation-based cathodes for AIBs. On this basis, Kaveevivitchai et al. ${ }^{80}$ used microwave-assisted hydrothermal synthesis to fabricate a molybdenum-vanadium mixed oxide, with an open structure and channels up to $6 \AA$ in diameter, allowing the fast and reversible insertion of $\mathrm{Al}^{3+}$. The device showed a remarkable discharge capacity at slow rates (up to $20 \mathrm{~mA} \mathrm{~g}^{-1}$ ), but a considerable loss in capacity at higher rates. Furthermore, no long-term cycling data was provided, suggesting that the material might have a limited cycling life.

Halogens have also recently been investigated as cathode materials, exploiting a conversion reaction mechanism. A preliminary study on the feasibility of a rechargeable $\mathrm{Al}-\mathrm{Br}_{2}$ battery was conducted by Hog et al. ${ }^{81}$ The battery featured a device design with two half-cells separated by a membrane, using both an anolyte and a catholyte. Unfortunately, the experimental data was not in good agreement with the theoretical predictions made by the authors, and the device could not be effectively recharged. A more successful report was reported using elemental iodine as a cathode material, as presented by Zhang et al. ${ }^{82}$ The authors proposed a polyvinylpyrrolidone (PVP)-iodine compound, absorbed on carbon cloth, as the cathode. The advantage of such composite material is its ability to effectively inhibit the shuttling of polyiodide ions from cathode to anode, a process similar to what happens in Al-S battery chemistry, resulting in efficiency losses and self-discharge. Thanks to this effect, the device could achieve remarkable specific capacities with good coulombic efficiency and cycle life.

\subsection{Electrolytes}

\subsubsection{Alkyl-imidazolium chloride melts}

Most literature published so far recognises the combination of $\mathrm{AlCl}_{3}$ and [EMIm] Cl as the most effective electrolyte system for AIBs. Originally, this electrolyte was proposed as part of a cheaper, environmentally friendly technique for the electrodeposition of aluminium, with the aim of using it as an alternative to more 
inefficient and energetically expensive electrolysis processes such as the HallHéroult method. ${ }^{23}$ Progress in the field of room temperature ionic liquids led to the publication of a number of works proposing ionic liquids as electrolytes for the electrodeposition of aluminium. ${ }^{52}$ These electrolytes were generally formed by the combination of $\mathrm{AlCl}_{3}$ and a chloride salt of an organic cation with a lowtemperature melting point, such as n-butylpyridinium chloride ${ }^{83}$ trimethyl-phenylammonium chloride, ${ }^{84}$ and benzyl-triphenyl-ammonium chloride.$^{85}$ Most of them, however, were restricted by severe limitations such as low conductivities, high viscosities, and narrow electrochemical stability windows. [EMIm] $\mathrm{Cl}$, on the other hand, showed an electrochemical stability window of over $3 \mathrm{~V}$, acceptable viscosity, and relatively high conductivity. One of the earliest works on the subject was published by Auborn and Barberio, ${ }^{22}$ who first demonstrated the reversibility of the electrodeposition reaction, using both in a "thin film" cell and a flooded 3 electrode cell, and employing a tungsten working electrode. The work only explored the rates of galvanostatic deposition as a function of the melt composition (i.e. the molar fraction of $\mathrm{AlCl}_{3}$ in the electrolyte), without providing much data on voltages, or the mechanisms involved in the process. The authors were also among the first to speculate on the possibility of using this system for a secondary battery. Shortly after, Lai and Skyllas-Kazacos also showed how acidic mixtures could yield efficient aluminium electrodeposition on inert substrates such as platinum, gold, tungsten, or glassy carbon. ${ }^{53}$ Despite the considerable volume of works on this topic, there were initially few reports of electrodeposition of $\mathrm{Al}$ on $\mathrm{Al}$ substrates. ${ }^{85,86}$ Within this category, one of the most cited papers in recent AIB literature is the publication by Jiang et al. ${ }^{23}$ In this work, the authors investigated the electrodeposition reaction in deeper detail by examining the surface morphology of the deposits, and studied the kinetics of the process by performing chronoamperometric measurements.

\section{$\mathrm{AlCl}_{3} /[\mathrm{EMIm}] \mathrm{Cl}$ molar ratios}

Although the $\mathrm{AlCl}_{3} /[\mathrm{EMIm}] \mathrm{Cl}$ complex appears to have been unanimously chosen as the best choice of electrolyte for AIBs, there seems to be a bit of controversy between publications on the optimal molar composition of the two components. As previously mentioned, in order for the electrodeposition reaction to happen, the molar fraction of $\mathrm{AlCl}_{3}$ in the mixture needs to be greater than 0.5 (giving it 
Lewis acid characteristics), so that the $\mathrm{Al}_{2} \mathrm{Cl}_{7}^{-}$ion is present in the solution. ${ }^{52}$ It is worth noting that, technically, electrodeposition could also take place from the reduction of the $\mathrm{AlCl}_{4}^{-}$ion (i.e. in eutectic mixtures where the molar fraction of $\mathrm{AlCl}_{3}$ is less than 0.5), but it requires a more negative potential than the one required to reduce the organic [EMIm] ${ }^{+}$cation. ${ }^{87}$ Early AIB publications report the use of different electrolyte ratios in their devices, without focusing much on the optimisation of this parameter. The original work by Paranthaman et al. ${ }^{26}$ reports the use of a 2:1 ratio for the electrolyte, but does not mention the reasoning behind such choice, which might just be attributed to a simple replication of the system applied by Jiang et al. ${ }^{23}$ Jayaprakash et al. ${ }^{27}$ later found to obtain better results with a 1.1:1 molar ratio, but unfortunately no experimental evidence is provided to support the claim. Oddly enough, Reed and Menke's rebuttal work instead used a 1.2:1 ratio instead, again providing no justification for this choice. ${ }^{28}$ The $\mathrm{Al}-\mathrm{VO}_{2}$ cell proposed by Wang et al. ${ }^{33}$ used a 1:1 mixture, which could be one of the reasons for the underwhelming performances of their device. Finally, the chloroaluminate-doped polymer AIB proposed by Hudak ${ }^{60}$ went for a 1.5:1 molar ratio.

The first proper study on the optimisation of the electrolyte molar ratio can be found in Lin et al.'s publication on the ultrafast $\mathrm{Al} /$ graphitic foam battery. ${ }^{38}$ The authors tested out a variety of ratios ranging from 1.1 to 1.8 in a Swagelok-type cell, and found that compositions around the 1.3-1.5 mark yielded the highest specific capacities relative to the graphite electrode. ${ }^{38}$ Later, a report by Wang et al. ${ }^{88}$ focused on the anion-effect of ionic liquid electrolytes in AIBs found that, for a $\mathrm{V}_{2} \mathrm{O}_{5}$ nanowire cathode and an $\mathrm{AlCl}_{3}$ /1-butyl-3-methylimidazolium (BMIm) chloride electrolyte, the best performance was shown when using a 1.1:1 ratio. All the previously mentioned reports, however, do not account for the capacitylimiting effect of the electrolyte proposed by Kravchyk et al., which was described in the previous section. ${ }^{46}$ When taking this principle into account, it was found that the highest achievable concentration of $\mathrm{AlCl}_{3}$ will always give rise to the highest theoretical specific energy density in the cell, even considering the reduced specific cathodic capacity. Naturally, this principle only applies for graphitic cathodes, where the intercalation of $\mathrm{AlCl}_{4}^{-}$species takes place during the charging phase. When using $\mathrm{V}_{2} \mathrm{O}_{5}$ as cathode (or any material employing a similar reaction mechanism), the accepted mechanism is that $\mathrm{Al}^{3+}$ ions, not $\mathrm{AlCl}_{4}^{-}$, intercalate 
at the cathode during the discharge process instead of the charge, according to the following mechanism: ${ }^{32}$

$$
\begin{aligned}
& \text { Cathode: } \quad 4 \mathrm{Al}_{2} \mathrm{Cl}_{7}^{-}+\mathrm{x} \mathrm{V}_{2} \mathrm{O}_{5}+3 \mathrm{e}^{-} \rightleftharpoons \mathrm{Al}\left(\mathrm{V}_{2} \mathrm{O}_{5}\right)_{\mathrm{x}}+7 \mathrm{AlCl}_{4}^{-} \\
& \text {Anode: } \\
& 7 \mathrm{AlCl}_{4}^{-}+\mathrm{Al} \rightleftharpoons 4 \mathrm{Al}_{2} \mathrm{Cl}_{7}^{-}+3 \mathrm{e}^{-}
\end{aligned}
$$

Based on Equations 2.5a and 2.5b, the $\mathrm{Al}_{2} \mathrm{Cl}_{7}^{-}$ions produced at the anode during the discharge get consumed at the cathode, thus creating a unilateral flow of Alcontaining species from anode to cathode. Combining the above equations, the full-cell reaction can be written as:

$$
\mathrm{Al}+\mathrm{xV}_{2} \mathrm{O}_{5} \rightleftharpoons \mathrm{Al}\left(\mathrm{V}_{2} \mathrm{O}_{5}\right)_{\mathrm{x}}
$$

It is evident that none of the species constituting the electrolyte appear in Equation 2.6, and only the anodic and cathodic materials feature. Therefore, assuming that the above equation is correct, the capacity limiting aspect of the electrolyte does not manifest in this type of cell architecture.

Recently, a publication by Ferrara et al. also investigated the optimal molar ratio of the electrolytes by characterising the $\mathrm{AlCl}_{3} /[\mathrm{EMIm}] \mathrm{Cl}$ mixture, in a wide range of molar ratios going from 1.1 to 1.7 , using a wide variety of physico-chemical techniques, and using $\mathrm{V}_{2} \mathrm{O}_{5}$ as the cathode material. ${ }^{89}$ The authors conclude that, due to the highest ionic conductivity and concentration of $\mathrm{Al}_{2} \mathrm{Cl}_{7}^{-}$ions, the optimal molar ratio for the electrolyte is 1.2. Galvanostatic charge-discharge tests, however, reveal no significant difference in specific discharge capacities between any of the molar ratios tested, indicating that the properties observed in the characterisation studies possibly have no relevant impact on battery performance.

Based on the aforementioned publications, a certain degree of controversy on what is the optimal $\mathrm{AlCl}_{3} /[\mathrm{EMIm}] \mathrm{Cl}$ molar ratio for AIB electrolytes still exists in the literature. This controversy, however, could simply depend on the diverse experimental conditions in which the electrolytes were tested, such as different cathode materials and cell designs. Nevertheless, most studies tend to agree that a ratio between 1.1 and 1.3 can provide good performance, and the variation within such range can be somewhat negligible. 


\subsubsection{Alternative electrolytes}

Although the $[\mathrm{EMIm}] \mathrm{Cl} / \mathrm{AlCl}_{3}$ eutectic is the most popular electrolyte choice, some alternative systems have been proposed. One interesting variation was proposed by Sun et al. who synthesised a polymer gel electrolyte using $\mathrm{AlCl}_{3^{-}}$ complexed acrylamide as the starting monomer and used a mixture of [EMIm]Cl/ $\mathrm{AlCl}_{3}$ as plasticiser..$^{90}$ Polymer gels can have a shielding effect from the moisture, and therefore alleviate the problem of water sensitivity of the electrolyte. Indeed, the polymer gel presented in this work displayed reversible electrochemical Alplating activity on a $\mathrm{Cu}$ substrate, even after 10 minutes of ambient exposure. On the other hand, the same experiment performed using [EMIm] Cl/ $\mathrm{AlCl}_{3}$ showed no electrochemical activity whatsoever after only 3 minutes of air exposure. Unfortunately, the authors did not report the performance of this electrolyte in fully assembled cells, or using any known cathode materials. More recently, a report by Yu et al. ${ }^{91}$ successfully used a polyacrylamide gel to incorporate the electrolyte and used it in an Al-graphite battery.

Urea is known to form a liquid melt when combined with $\mathrm{AlCl}_{3}$. One recent report demonstrated its ability to reversibly plate aluminium under ambient conditions. ${ }^{92}$ On this basis, Jiao et al. ${ }^{93}$ proposed an Al-graphite cell using a urea $/ \mathrm{AlCl}_{3}$ electrolyte. The main issue arising from this work is the need to operate the cell at $120^{\circ} \mathrm{C}$. The authors claimed that despite the possibility of electrodepositing $\mathrm{Al}$ under ambient conditions claimed by previous literature, ${ }^{92}$ the conductivity of the system was too low to operate efficiently at room temperature. Nevertheless, the device still showed remarkable specific capacity and cycling stability. A similar approach was adopted by Song et al., ${ }^{94}$ who developed a fully inorganic electrolyte system based on a $\mathrm{NaCl} / \mathrm{AlCl}_{3}$ eutectic mixture for AIBs. Using a graphitic carbon paper electrode, and operating at $120^{\circ} \mathrm{C}$, the cell showed excellent cycling stability and a rather high specific capacity. The authors attributed the remarkable performance of the cell to the high conductivity of the electrolyte melt. Another alternative electrolyte system for AIBs was proposed by Wang et al., who investigated the combination of aluminium trifluoromethanesulfonate (Al(OTf $\left.)_{3}\right)$ and 1-butyl3-imidazolium triflate ([BMIM]OTf) for an $\mathrm{Al} / \mathrm{V}_{2} \mathrm{O}_{5}$ battery. ${ }^{95}$ The main rationale behind this study was to avoid the need to use the highly moisture-sensitive and corrosive $[\mathrm{EMIm}] \mathrm{Cl} / \mathrm{AlCl}_{3}$ ionic electrolyte. The authors argued that due of the 
non-corrosiveness of the trifluoromethanesulfonate-based electrolyte, the $\mathrm{Al}$ anode needed be pre-treated in a 1.1:1 $\mathrm{AlCl}_{3} /[\mathrm{BMIm}] \mathrm{Cl}$ mixture for 24 hours, to remove the $\mathrm{Al}_{2} \mathrm{O}_{3}$ film on its surface and allow the plating and stripping foil of $\mathrm{Al}$ metal.

Recent publications have also focused on finding low-cost alternatives to the conventional imidazolium chloride salts, with comparable or even improved performance. Our research group has recently released a paper on the use of acetamide as a substitute to [EMIm]Cl, with comparable performance. ${ }^{96}$ The findings of this publications are described in detail in Chapter 8. In another publication, Xu et al. reported the use of triethylamine hydrochloride, a very cheap compound and intractable industrial waste, as the alternative to [EMIm]Cl. The electrolyte proposed by these authors also has the advantage of displaying a wider electrochemical window compared to [EMIm]Cl, which could potentially lead to better performance by allowing deeper charging levels. ${ }^{97}$

\section{Bibliography}

${ }^{1}$ W. S. Harris, "Electrochemical studies in cyclic esters,"

${ }^{2}$ Yung-Fang Yu Yao and J. T. Kummer, "Ion exchange properties of and rates of ionic diffusion in beta-alumina," Journal of Inorganic and Nuclear Chemistry, vol. 29, pp. 2453-2475, Sept. 1967.

${ }^{3} \mathrm{~J}$. Newman, "Transport processes in electrolytic solutions," Advances in Electrochemistry and Electrochemical Engineering, vol. 5, pp. 87-136, 1967.

${ }^{4}$ M. S. Whittingham, "Electrointercalation in transition-metal disulphides," Journal of the Chemical Society, Chemical Communications, pp. 328-329, Jan. 1974.

${ }^{5}$ M. S. Whittingham, "History, Evolution, and Future Status of Energy Storage," Proceedings of the IEEE, vol. 100, pp. 1518-1534, May 2012.

${ }^{6} \mathrm{M}$. Armand and P. Touzain, "Graphite intercalation compounds as cathode materials," Materials Science and Engineering, vol. 31, pp. 319-329, Dec. 1977.

${ }^{7}$ K. Mizushima, P. C. Jones, P. J. Wiseman, and J. B. Goodenough, "LixCoO2 $(0<x<-1)$ : A new cathode material for batteries of high energy density," Materials Research Bulletin, vol. 15, pp. 783789, June 1980.

${ }^{8}$ A. Yoshino, "The Birth of the Lithium-Ion Battery," Angewandte Chemie International Edition, vol. 51, no. 24, pp. 5798-5800, 2012.

${ }^{9}$ E. Peled, "The Electrochemical Behavior of Alkali and Alkaline Earth Metals in Nonaqueous Battery Systems-The Solid Electrolyte Interphase Model," Journal of The Electrochemical Society, vol. 126, pp. 2047-2051, Jan. 1979. 
${ }^{10}$ R. Fong, U. v. Sacken, and J. R. Dahn, "Studies of Lithium Intercalation into Carbons Using Nonaqueous Electrochemical Cells," Journal of The Electrochemical Society, vol. 137, pp. 20092013, Jan. 1990.

${ }^{11}$ M. M. Thackeray, W. I. F. David, P. G. Bruce, and J. B. Goodenough, "Lithium insertion into manganese spinels," Materials Research Bulletin, vol. 18, pp. 461-472, Apr. 1983.

${ }^{12}$ A. K. Padhi, K. S. Nanjundaswamy, and J. B. Goodenough, "Phospho-olivines as PositiveElectrode Materials for Rechargeable Lithium Batteries," Journal of The Electrochemical Society, vol. 144, pp. 1188-1194, Jan. 1997.

${ }^{13}$ A. des sciences (France), A. d. s. (France), and C. n. d. l. r. s. (France), Comptes rendus hebdomadaires des séances de l'Académie des sciences., vol. t.40 (1855). Paris: publiés avec le concours du Centre national de la recherche scientifique par MM. les secrétaires perpétuels :, 1855.

${ }^{14}$ R. Samuel, "Primary cell," May 1953. U.S. Classification 429/199, 429/224; International Classification H01M6/04; Cooperative Classification H01M6/04, Y02E60/12; European Classification H01M6/04.

${ }^{15}$ S. Zaromb, "The Use and Behavior of Aluminum Anodes in Alkaline Primary Batteries," Journal of The Electrochemical Society, vol. 109, pp. 1125-1130, Jan. 1962.

${ }^{16}$ L. Bockstie, D. Trevethan, and S. Zaromb, "Control of Al Corrosion in Caustic Solutions," Journal of The Electrochemical Society, vol. 110, pp. 267-271, Jan. 1963.

${ }^{17} \mathrm{Q}$. Li and N. J. Bjerrum, "Aluminum as anode for energy storage and conversion: a review," Journal of Power Sources, vol. 110, pp. 1-10, July 2002.

${ }^{18}$ Y. Tang, L. Lu, H. W. Roesky, L. Wang, and B. Huang, "The effect of zinc on the aluminum anode of the aluminum-air battery," Journal of Power Sources, vol. 138, pp. 313-318, Nov. 2004.

${ }^{19}$ R. J. Gale and R. A. Osteryoung, "Potentiometric investigation of dialuminum heptachloride formation in aluminum chloride-1-butylpyridinium chloride mixtures," Inorganic Chemistry, vol. 18, pp. 1603-1605, June 1979.

${ }^{20}$ J. S. Wilkes, J. A. Levisky, R. A. Wilson, and C. L. Hussey, “Dialkylimidazolium chloroaluminate melts: a new class of room-temperature ionic liquids for electrochemistry, spectroscopy and synthesis," Inorganic Chemistry, vol. 21, pp. 1263-1264, Mar. 1982.

${ }^{21}$ G. F. Reynolds and C. J. Dymek, "Primary and secondary room temperature molten salt electrochemical cells," Journal of Power Sources, vol. 15, pp. 109-118, June 1985.

${ }^{22}$ J. J. Auborn and Y. L. Barberio, "An Ambient Temperature Secondary Aluminum Electrode: Its Cycling Rates and Its Cycling Efficiencies," Journal of The Electrochemical Society, vol. 132, pp. 598601, Jan. 1985.

${ }^{23}$ T. Jiang, M. J. Chollier Brym, G. Dubé, A. Lasia, and G. M. Brisard, “Electrodeposition of aluminium from ionic liquids: Part I-electrodeposition and surface morphology of aluminium from aluminium chloride ( $\mathrm{AlCl}$ )-1-ethyl-3-methylimidazolium chloride ([EMIm]Cl) ionic liquids," Surface and Coatings Technology, vol. 201, pp. 1-9, Sept. 2006.

${ }^{24}$ P. R. Gifford and J. B. Palmisano, "An Aluminum/Chlorine Rechargeable Cell Employing a Room Temperature Molten Salt Electrolyte," Journal of The Electrochemical Society, vol. 135, pp. 650-654, Mar. 1988.

${ }^{25}$ F. M. Donahue, S. E. Mancini, and L. Simonsen, "Secondary aluminium-iron (III) chloride batteries with a low temperature molten salt electrolyte," Journal of Applied Electrochemistry, vol. 22, pp. 230-234, Mar. 1992. 
${ }^{26}$ M. P. Paranthaman, G. Brown, X.-G. Sun, J. Nanda, A. Manthiram, and A. Manivannan, "A Transformational, High Energy Density, Secondary Aluminum Ion Battery," Meeting Abstracts, vol. MA2010-02, pp. 314-314, Aug. 2010.

${ }^{27}$ N. Jayaprakash, S. K. Das, and L. A. Archer, "The rechargeable aluminum-ion battery," Chemical Communications, vol. 47, no. 47, pp. 12610-12612, 2011.

${ }^{28}$ L. D. Reed and E. Menke, "The Roles of V2O5 and Stainless Steel in Rechargeable Al-Ion Batteries," Journal of the Electrochemical Society, vol. 160, no. 6, pp. A915-A917, 2013.

${ }^{29}$ F. Chaput, B. Dunn, P. Fuqua, and K. Salloux, "Synthesis and characterization of vanadium oxide aerogels," Journal of Non-Crystalline Solids, vol. 188, pp. 11-18, July 1995.

${ }^{30}$ H. Wang, Y. Bai, S. Chen, X. Luo, C. Wu, F. Wu, J. Lu, and K. Amine, “Binder-Free V2O5 Cathode for Greener Rechargeable Aluminum Battery," ACS Applied Materials \& Interfaces, vol. 7, pp. 8084, Jan. 2015.

${ }^{31}$ M. Chiku, H. Takeda, S. Matsumura, E. Higuchi, and H. Inoue, "Amorphous Vanadium Oxide/Carbon Composite Positive Electrode for Rechargeable Aluminum Battery," ACS Applied Materials \& Interfaces, vol. 7, pp. 24385-24389, Nov. 2015.

${ }^{32} \mathrm{~S}$. Gu, H. Wang, C. Wu, Y. Bai, H. Li, and F. Wu, "Confirming reversible Al3+ storage mechanism through intercalation of $\mathrm{Al} 3+$ into $\mathrm{V} 2 \mathrm{O} 5$ nanowires in a rechargeable aluminum battery," Energy Storage Materials, vol. 6, pp. 9-17, Jan. 2017.

${ }^{33}$ W. Wang, B. Jiang, W. Xiong, H. Sun, Z. Lin, L. Hu, J. Tu, J. Hou, H. Zhu, and S. Jiao, “A new cathode material for super-valent battery based on aluminium ion intercalation and deintercalation," Scientific Reports, vol. 3, Nov. 2013.

${ }^{34}$ J. V. Rani, V. Kanakaiah, T. Dadmal, M. S. Rao, and S. Bhavanarushi, "Fluorinated Natural Graphite Cathode for Rechargeable Ionic Liquid Based Aluminum-Ion Battery," Journal of The Electrochemical Society, vol. 160, pp. A1781-A1784, Jan. 2013.

${ }^{35}$ T. Nakajima, M. Koh, V. Gupta, B. Žemva, and K. Lutar, “Electrochemical behavior of graphite highly fluorinated by high oxidation state complex fluorides and elemental fluorine," Electrochimica Acta, vol. 45, pp. 1655-1661, Jan. 2000.

${ }^{36}$ G. Levitin, C. Yarnitzky, and S. Licht, "Fluorinated Graphites as Energetic Cathodes for Nonaqueous Al Batteries," Electrochemical and Solid-State Letters, vol. 5, pp. A160-A163, Jan. 2002.

${ }^{37}$ H. Sun, W. Wang, Z. Yu, Y. Yuan, S. Wang, and S. Jiao, “A new aluminium-ion battery with high voltage, high safety and low cost," Chemical Communications, vol. 51, pp. 11892-11895, July 2015.

${ }^{38}$ M.-C. Lin, M. Gong, B. Lu, Y. Wu, D.-Y. Wang, M. Guan, M. Angell, C. Chen, J. Yang, B.-J. Hwang, and H. Dai, "An ultrafast rechargeable aluminium-ion battery," Nature, vol. 520, pp. 324-328, Apr. 2015.

${ }^{39}$ M. Fouletier and M. Armand, "Electrochemical method for characterization of graphitealuminium chloride intercalation compounds," Carbon, vol. 17, pp. 427-429, Jan. 1979.

${ }^{40}$ A. Baiker, E. Habegger, and R. Schlögl, "Intercalation of Graphite by Aluminum Chloride. Influence of Graphite Properties on Intercalation Rate," Berichte der Bunsengesellschaft für physikalische Chemie, vol. 89, pp. 530-538, May 1985.

${ }^{41}$ Y. Yang, C. Han, B. Jiang, J. Iocozzia, C. He, D. Shi, T. Jiang, and Z. Lin, “Graphene-based materials with tailored nanostructures for energy conversion and storage," Materials Science $\mathcal{E}$ Engineering R-Reports, vol. 102, pp. 1-72, Apr. 2016. WOS:000374359900001. 
${ }^{42}$ X. Yu, B. Wang, D. Gong, Z. Xu, and B. Lu, "Graphene Nanoribbons on Highly Porous 3d Graphene for High-Capacity and Ultrastable Al-Ion Batteries," Advanced Materials, vol. 29, p. 1604118, Jan. 2017.

${ }^{43}$ N. P. Stadie, S. Wang, K. V. Kravchyk, and M. V. Kovalenko, "Zeolite-Templated Carbon as an Ordered Microporous Electrode for Aluminum Batteries," ACS Nano, vol. 11, pp. 1911-1919, Feb. 2017.

${ }^{44}$ S. Wang, K. V. Kravchyk, F. Krumeich, and M. V. Kovalenko, “Kish Graphite Flakes as a Cathode Material for an Aluminum Chloride-Graphite Battery," ACS Applied Materials \& Interfaces, vol. 9, pp. 28478-28485, Aug. 2017.

${ }^{45}$ D.-Y. Wang, C.-Y. Wei, M.-C. Lin, C.-J. Pan, H.-L. Chou, H.-A. Chen, M. Gong, Y. Wu, C. Yuan, M. Angell, Y.-J. Hsieh, Y.-H. Chen, C.-Y. Wen, C.-W. Chen, B.-J. Hwang, C.-C. Chen, and H. Dai, "Advanced rechargeable aluminium ion battery with a high-quality natural graphite cathode," Nature Communications, vol. 8, p. 14283, Feb. 2017.

${ }^{46}$ K. V. Kravchyk, S. Wang, L. Piveteau, and M. V. Kovalenko, "Efficient Aluminum Chloride-Natural Graphite Battery," Chemistry of Materials, vol. 29, pp. 4484-4492, May 2017.

${ }^{47}$ S. Wang, K. Kravchyk, A. Filippin, U. Müller, A. Tiwari, S. Buecheler, M. Bodnarchuk, and M. Kovalenko, "Aluminum Chloride-Graphite Batteries with Flexible Current Collectors Prepared from Earth-Abundant Elements," Advanced Science, vol. 5, no. 4, 2018.

${ }^{48}$ G. Antonio Elia, I. Hasa, G. Greco, T. Diemant, K. Marquardt, K. Hoeppner, R. Jürgen Behm, A. Hoell, S. Passerini, and R. Hahn, "Insights into the reversibility of aluminum graphite batteries," Journal of Materials Chemistry A, vol. 5, no. 20, pp. 9682-9690, 2017.

${ }^{49}$ Z. Li, B. Niu, Y. Liu, J. Li, and F. Kang, "Prelithiation treatment of graphite as cathode material for rechargeable aluminum batteries," Electrochimica Acta, vol. 263, pp. 68-75, Feb. 2018.

${ }^{50}$ S. Wang, S. Jiao, W.-L. Song, H.-S. Chen, J. Tu, D. Tian, H. Jiao, C. Fu, and D.-N. Fang, “A novel dual-graphite aluminum-ion battery," Energy Storage Materials, vol. 12, pp. 119-127, 2018.

${ }^{51}$ P. K. Lai and M. Skyllas-Kazacos, "Aluminium deposition and dissolution in aluminium chloride-n-butylpyridinium chloride melts," Electrochimica Acta, vol. 32, pp. 1443-1449, Oct. 1987.

${ }^{52}$ Y. Zhao and T. J. VanderNoot, "Electrodeposition of aluminium from nonaqueous organic electrolytic systems and room temperature molten salts," Electrochimica Acta, vol. 42, pp. 3-13, Jan. 1997.

${ }^{53}$ P. K. Lai and M. Skyllas-Kazacos, "Electrodeposition of aluminium in aluminium chloride/1methyl-3-ethylimidazolium chloride," Journal of Electroanalytical Chemistry and Interfacial Electrochemistry, vol. 248, pp. 431-440, July 1988.

${ }^{54}$ M. S. Wu, B. Xu, L. Q. Chen, and C. Y. Ouyang, "Geometry and fast diffusion of AlCl4 cluster intercalated in graphite," Electrochimica Acta, vol. 195, pp. 158-165, Mar. 2016.

${ }^{55}$ G. Greco, D. Tatchev, A. Hoell, M. Krumrey, S. Raoux, R. Hahn, and G. Antonio Elia, “Influence of the electrode nano/microstructure on the electrochemical properties of graphite in aluminum batteries," Journal of Materials Chemistry A, vol. 6, no. 45, pp. 22673-22680, 2018.

${ }^{56}$ H. P. Boehm, R. Setton, and E. Stumpp, "Nomenclature and terminology of graphite intercalation compounds (IUPAC Recommendations 1994)," Pure and Applied Chemistry, vol. 66, no. 9, pp. 1893-1901, 2009.

${ }^{57}$ A. S. Childress, P. Parajuli, J. Zhu, R. Podila, and A. M. Rao, "A Raman spectroscopic study of graphene cathodes in high-performance aluminum ion batteries," Nano Energy, vol. 39, pp. 6976, Sept. 2017. 
${ }^{58}$ P. Bhauriyal, A. Mahata, and B. Pathak, "The staging mechanism of AlCl4- intercalation in a graphite electrode for an aluminium-ion battery," Physical Chemistry Chemical Physics, vol. 19, pp. 7980-7989, Mar. 2017.

${ }^{59}$ C.-J. Pan, C. Yuan, G. Zhu, Q. Zhang, C.-J. Huang, M.-C. Lin, M. Angell, B.-J. Hwang, P. Kaghazchi, and H. Dai, "An operando X-ray diffraction study of chloroaluminate anion-graphite intercalation in aluminum batteries," Proceedings of the National Academy of Sciences, vol. 115, pp. 5670-5675, May 2018.

${ }^{60}$ N. S. Hudak, "Chloroaluminate-Doped Conducting Polymers as Positive Electrodes in Rechargeable Aluminum Batteries," The Journal of Physical Chemistry C, vol. 118, pp. 5203-5215, Mar. 2014.

${ }^{61}$ M. Walter, K. V. Kravchyk, C. Böfer, R. Widmer, and M. V. Kovalenko, “Polypyrenes as HighPerformance Cathode Materials for Aluminum Batteries," Advanced Materials, vol. 30, no. 15, p. 1705644, 2018.

${ }^{62}$ X. Fan, F. Wang, X. Ji, R. Wang, T. Gao, S. Hou, J. Chen, T. Deng, X. Li, L. Chen, C. Luo, L. Wang, and C. Wang, "A Universal Organic Cathode for Ultrafast Lithium and Multivalent Metal Batteries," Angewandte Chemie International Edition, vol. 57, pp. 7146-7150, June 2018.

${ }^{63}$ D. J. Kim, D.-J. Yoo, M. T. Otley, A. Prokofjevs, C. Pezzato, M. Owczarek, S. J. Lee, J. W. Choi, and J. F. Stoddart, "Rechargeable aluminium organic batteries," Nature Energy, p. 1, Dec. 2018.

${ }^{64}$ Y. Liang and Y. Yao, "Halfway through," Nature Energy, p. 1, Dec. 2018.

${ }^{65}$ L. Mei, J. Xu, Z. Wei, H. Liu, Y. Li, J. Ma, and S. Dou, "Chevrel Phase Mo6t8 (T = S, Se) as Electrodes for Advanced Energy Storage," Small, vol. 13, no. 34, 2017.

${ }^{66}$ L. Geng, G. Lv, X. Xing, and J. Guo, "Reversible Electrochemical Intercalation of Aluminum in Mo6s8," Chemistry of Materials, vol. 27, pp. 4926-4929, July 2015.

${ }^{67}$ Z. Li, B. Niu, J. Liu, J. Li, and F. Kang, "Rechargeable Aluminum-Ion Battery Based on MoS2 Microsphere Cathode," ACS Applied Materials and Interfaces, vol. 10, no. 11, pp. 9451-9459, 2018.

${ }^{68}$ L. Wu, R. Sun, F. Xiong, C. Pei, K. Han, C. Peng, Y. Fan, W. Yang, Q. An, and L. Mai, "A rechargeable aluminum-ion battery based on a VS2 nanosheet cathode," Physical Chemistry Chemical Physics, vol. 20, pp. 22563-22568, Sept. 2018.

${ }^{69}$ H. Li, H. Yang, Z. Sun, Y. Shi, H.-M. Cheng, and F. Li, “A highly reversible Co3s4 microsphere cathode material for aluminum-ion batteries," Nano Energy, vol. 56, pp. 100-108, Feb. 2019.

${ }^{70}$ W. Xing, D. Du, T. Cai, X. Li, J. Zhou, Y. Chai, Q. Xue, and Z. Yan, “Carbon-encapsulated CoSe nanoparticles derived from metal-organic frameworks as advanced cathode material for Al-ion battery," Journal of Power Sources, vol. 401, pp. 6-12, Oct. 2018.

${ }^{71}$ Y. Hu, D. Ye, B. Luo, H. Hu, X. Zhu, S. Wang, L. Li, S. Peng, and L. Wang, “A Binder-Free and Free-Standing Cobalt Sulfide@Carbon Nanotube Cathode Material for Aluminum-Ion Batteries," Advanced Materials, vol. 30, p. 1703824, Jan. 2018.

${ }^{72}$ K. Liang, L. Ju, S. Koul, A. Kushima, and Y. Yang, "Self-Supported Tin Sulfide Porous Films for Flexible Aluminum-Ion Batteries." 2018.

${ }^{73}$ J. Jiang, H. Li, T. Fu, B.-J. Hwang, X. Li, and J. Zhao, "One-Dimensional Cu2-xSe Nanorods as the Cathode Material for High-Performance Aluminum-Ion Battery," ACS Applied Materials $\mathcal{E}$ Interfaces, vol. 10, pp. 17942-17949, May 2018.

${ }^{74}$ G. Cohn, L. Ma, and L. A. Archer, "A novel non-aqueous aluminum sulfur battery," Journal of Power Sources, vol. 283, pp. 416-422, June 2015. 
${ }^{75}$ T. Mori, Y. Orikasa, K. Nakanishi, C. Kezheng, M. Hattori, T. Ohta, and Y. Uchimoto, “Discharge/charge reaction mechanisms of FeS2 cathode material for aluminum rechargeable batteries at $55^{\circ} \mathrm{C}$," Journal of Power Sources, vol. 313, pp. 9-14, May 2016.

${ }^{76} \mathrm{X}$. Yu and A. Manthiram, "Electrochemical Energy Storage with a Reversible Nonaqueous Room-Temperature Aluminum-Sulfur Chemistry," Advanced Energy Materials, vol. 7, p. 1700561, Sept. 2017.

${ }^{77}$ X. Huang, Y. Liu, C. Liu, J. Zhang, O. Noonan, and C. Yu, "Rechargeable aluminum-selenium batteries with high capacity," Chemical Science, vol. 9, no. 23, pp. 5178-5182, 2018.

${ }^{78}$ A. VahidMohammadi, A. Hadjikhani, S. Shahbazmohamadi, and M. Beidaghi, "TwoDimensional Vanadium Carbide (MXene) as a High-Capacity Cathode Material for Rechargeable Aluminum Batteries," ACS Nano, vol. 11, pp. 11135-11144, Nov. 2017.

${ }^{79}$ M. Naguib, V. N. Mochalin, M. W. Barsoum, and Y. Gogotsi, “25th Anniversary Article: MXenes: A New Family of Two-Dimensional Materials," Advanced Materials, vol. 26, no. 7, pp. 992-1005, 2014.

${ }^{80}$ W. Kaveevivitchai, A. Huq, S. Wang, M. J. Park, and A. Manthiram, “Rechargeable AluminumIon Batteries Based on an Open-Tunnel Framework," Small, vol. 13, pp. n/a-n/a, Sept. 2017.

${ }^{81}$ M. Hog, B. Burgenmeister, K. Bromberger, M. Schuster, S. Riedel, and I. Krossing, "First Investigations Towards the Feasibility of an Al/Br2 Battery Based on Ionic Liquids," ChemElectroChem, vol. 4, no. 11, pp. 2934-2942, 2017.

${ }^{82}$ S. Zhang, X. Tan, Z. Meng, H. Tian, F. Xu, and W.-Q. Han, "Naturally abundant highperformance rechargeable aluminum/iodine batteries based on conversion reaction chemistry," Journal of Materials Chemistry A, vol. 6, no. 21, pp. 9984-9996, 2018.

${ }^{83} \mathrm{Y}$. Chao-Cheng, "Electrodeposition of aluminum in molten AlCl3-n-butylpyridinium chloride electrolyte," Materials Chemistry and Physics, vol. 37, pp. 355-361, May 1994.

${ }^{84}$ Y. Zhao and T. J. VanderNoot, "Electrodeposition of aluminium from room temperature AlCl3TMPAC molten salts," Electrochimica Acta, vol. 42, pp. 1639-1643, Jan. 1997.

${ }^{85}$ A. P. Abbott, C. A. Eardley, N. R. S. Farley, G. A. Griffith, and A. Pratt, "Electrodeposition of aluminium and aluminium/platinum alloys from $\mathrm{AlCl} 3 /$ benzyltrimethylammonium chloride room temperature ionic liquids," Journal of Applied Electrochemistry, vol. 31, pp. 1345-1350, Dec. 2001.

${ }^{86}$ N. Papageorgiou and F.-P. Emmenegger, "The effect of cosolvents and additives on the electrochemical properties of [(Me)3phn][Al2Cl7] melts," Electrochimica Acta, vol. 38, pp. 245-252, Feb. 1993.

${ }^{87}$ R. T. Carlin and R. A. Osteryoung, "Aluminum Anodization in a Basic Ambient Temperature Molten Salt," Journal of The Electrochemical Society, vol. 136, pp. 1409-1415, Jan. 1989.

${ }^{88} \mathrm{H}$. Wang, S. Gu, Y. Bai, S. Chen, N. Zhu, C. Wu, and F. Wu, "Anion-effects on electrochemical properties of ionic liquid electrolytes for rechargeable aluminum batteries," Journal of Materials Chemistry A, vol. 3, pp. 22677-22686, Nov. 2015.

${ }^{89}$ C. Ferrara, V. Dall'Asta, V. Berbenni, E. Quartarone, and P. Mustarelli, "Physicochemical Characterization of AlCl3-1-Ethyl-3-methylimidazolium Chloride Ionic Liquid Electrolytes for Aluminum Rechargeable Batteries," Journal of Physical Chemistry C, vol. 121, no. 48, pp. 26607-26614, 2017.

${ }^{90}$ X.-G. Sun, Y. Fang, X. Jiang, K. Yoshii, T. Tsuda, and S. Dai, “Polymer gel electrolytes for application in aluminum deposition and rechargeable aluminum ion batteries," Chemical Communications, vol. 52, pp. 292-295, Dec. 2015. 
${ }^{91}$ Z. Yu, S. Jiao, S. Li, X. Chen, W.-L. Song, T. Teng, J. Tu, H.-S. Chen, G. Zhang, and D.-N. Fang, "Flexible Stable Solid-State Al-Ion Batteries," Advanced Functional Materials, vol. 29, p. 1806799, Nov. 2018.

${ }^{92}$ A. P. Abbott, R. C. Harris, Y.-T. Hsieh, K. S. Ryder, and I.-W. Sun, “Aluminium electrodeposition under ambient conditions," Physical Chemistry Chemical Physics, vol. 16, pp. 14675-14681, June 2014.

${ }^{93} \mathrm{H}$. Jiao, C. Wang, J. Tu, D. Tian, and S. Jiao, "A rechargeable Al-ion battery: Al/molten $\mathrm{AlCl} 3$ -urea/graphite," Chemical Communications, vol. 53, no. 15, pp. 2331-2334, 2017.

${ }^{94}$ Y. Song, S. Jiao, J. Tu, J. Wang, Y. Liu, H. Jiao, X. Mao, Z. Guo, and D. J. Fray, “A long-life rechargeable $\mathrm{Al}$ ion battery based on molten salts," Journal of Materials Chemistry A, vol. 5, pp. 1282-1291, Jan. 2017.

${ }^{95}$ H. Wang, S. Gu, Y. Bai, S. Chen, F. Wu, and C. Wu, "High-Voltage and Noncorrosive Ionic Liquid Electrolyte Used in Rechargeable Aluminum Battery," ACS Applied Materials \& Interfaces, vol. 8, pp. 27444-27448, Oct. 2016.

${ }^{96}$ N. Canever, N. Bertrand, and T. Nann, "Acetamide: a low-cost alternative to alkyl imidazolium chlorides for aluminium-ion batteries," Chemical Communications, vol. 54, no. 83, pp. 1172511728, 2018.

${ }^{97}$ H. Xu, T. Bai, H. Chen, F. Guo, J. Xi, T. Huang, S. Cai, X. Chu, J. Ling, W. Gao, Z. Xu, and C. Gao, "Low-cost $\mathrm{AlCl}$ /Et3nhcl electrolyte for high-performance aluminum-ion battery," Energy Storage Materials, Aug. 2018. 
Chapter 3

Methods and techniques 



\subsection{Preface}

This chapter will briefly describe the main experimental methods and instrumental techniques used for the creation, characterisation and testing of the nanomaterials and electrolytes developed in this $\mathrm{PhD}$ project.

\subsection{Nanomaterials fabrication techniques}

The unusual mechanical, electronic, and optical properties of nanomaterials have always attracted a great deal of interest in materials research. Recently, these properties have been exploited to obtain interesting advancements in the field of energy conversion and storage, resulting in a wide range of performance enhancements. ${ }^{1}$

Among the large variety of nanomaterial families known, electrospun nanofibres have been mainly investigated as novel cathodes in this $\mathrm{PhD}$ project. Two main reasons are imputable for this choice: first, from a practical standpoint, this class of materials had been previously investigated within this research group. ${ }^{2}$ The availability of the required instrumentation and the previously acquired experience with the technique therefore helped in synthesising the materials efficiently. Furthermore, it was hypothesised that the particular morphology of nanofibres would be especially helpful in the creation of high-performance cathode materials. Specifically, the enhanced surface area would result in enhanced contact between the cathodes and the electrolyte; additionally, the highly anisotropic shape of would also promote a more directional conduction of charges in the material, resulting in a more conductive electrode.

The main techniques used to create and modify nanofibrous materials in this thesis will be described below.

\subsubsection{Electrospinning}

Electrospinning is a remarkably simple but highly effective nanomaterial fabrication technique. Its working principle is based on the electrostatic effect of a strong electric field acting on a viscous liquid. In a typical electrospinning setup, a viscous solution, usually containing a polymer, is extruded through a metallic 
needle, which is connected to a high voltage source, applying a high potential generally between 10 and $30 \mathrm{kV}$. The high electric field has a charging effect on the the droplet of solution exiting the syringe, which gets stretched into an elongated conical shape, known as the Taylor cone. If the voltage is high enough and the solution possesses the correct viscosity and electric properties, the surface tension in the droplet can be broken. The Taylor cone then further stretches into a wire, which accelerates out of the needle and flies towards a grounded collector surface. During this flight period, the solvent evaporates and the wire bends and splays several times, causing the wire to get progressively thinner. This eventually leads to the formation of fibrous sub-micrometer/nanometer sized objects, which continuously accumulate on the collector surface (Figure 3.1). Alignment of the nanofibres can also be induced either mechanically by using a rotating cylinder as the collector, or by modification of the electric field using a collector composed of two parallel plates, or even by applying an external magnetic field. ${ }^{3}$ The final product is a free-standing mat of nanofibres that can either be used as-prepared or receive further treatments and modifications. For instance, if the solution used to produce the fibres contains an inorganic precursor, an additional thermal treatment can be applied to remove the carrier polymer and convert the inorganic precursor into a ceramic or ceramic-like material. Using this method, a broad spectrum of nanofibrous materials can be fabricated, including transition metal oxides, ${ }^{2,4}$ graphitic and amorphous carbon, ${ }^{5}$ metallic nanofibres, ${ }^{6}$ or even more complex inorganic structures such as perovskites. ${ }^{7}$

One of the biggest strengths of electrospinning is the simplicity and low cost of the instrumentation used to fabricate the materials: a basic setup consisting of a syringe pump, high voltage source, and collector plate, can be purchased for as low as 3,000 NZ\$. The technique also has a lot of potential for industrial applications, thanks to its good up-scaling capabilities. Because of this, it has already found commercial applications in fields such as environment protection, electronics, biotechnology, pharmaceutics, and cosmetics. ${ }^{8}$

Although the instrumentation used to produce electrospun nanofibres is quite basic, there are many parameters that can be altered to optimise the final morphology of the product. These parameters can be divided in three main categories: 1) solution properties (such as choice of solvent, carrier polymer, precursors, and 


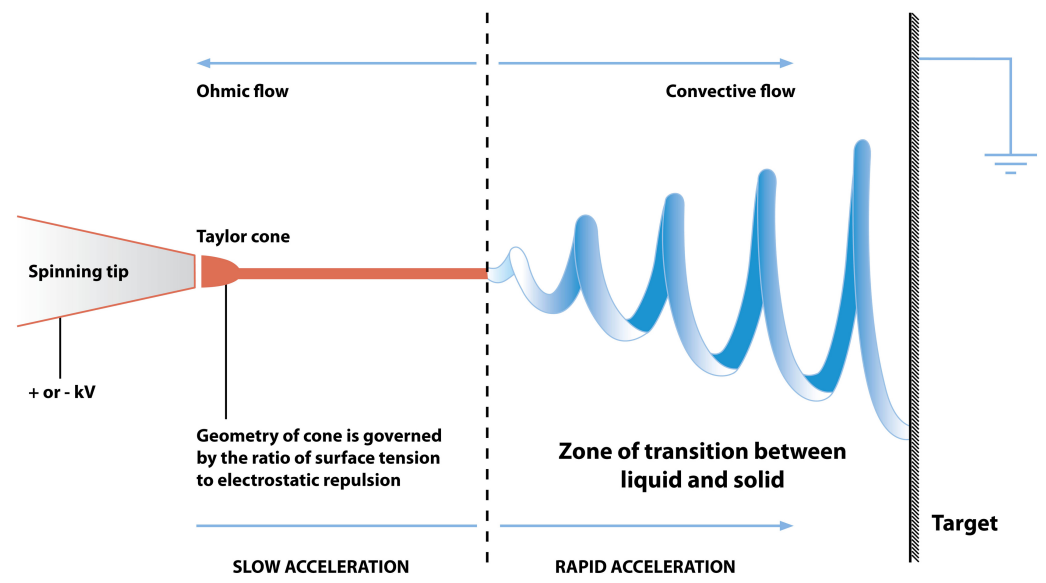

FIGURE 3.1: Diagram of the working principle of electrospinning (Image credits: Joanna Gatford - The New Zealand Institute for Plant and Food Research Ltd. Creative commons licence: CC BY 3.0).

their respective concentrations), 2) instrumental parameters (such as applied voltage, solution flow rate, nozzle diameter, and tip-to collector distance), and 3) environmental conditions (temperature and atmospheric humidity). Because of this, fabricating nanofibrous materials can sometimes be a challenging task, requiring many attempts before quality materials can be isolated. Nevertheless, the results are often worth the effort and can lead to interesting applications, making this fabrication technique very useful.

\section{Coaxial electrospinning}

An interesting evolution of this fabrication technique consists in using a special nozzle, featuring two concentric needles with different diameters. Two different solutions are fed through the needles, and are both affected by the strong electric field applied by the high voltage source. This modification allows the preparation of more complex nanostructures, such as core-sheath fibres ${ }^{9}$ or hollow nanotubes. ${ }^{10}$ Because two solutions are present, however, the number of variables to be optimised increases considerably, making the fabrication process even more complex. Nevertheless, if the optimal operating conditions are found, remarkable nanomaterials with different interesting properties can be obtained. ${ }^{11}$ 


\subsubsection{Hydrothermal and solvothermal synthesis}

This technique relies on the combination of temperature and pressure to promote the growth of materials that would otherwise either require much higher temperatures or could just not be prepared in ambient conditions. In addition, the special environments of this process can promote the preferential growth of crystalline materials along one particular crystallographic axis, which can also lead to the formation of anisotropic structures such as nanosheets and nanowires. ${ }^{12-14}$ This materials fabrication method is based on placing a solution containing inorganic salts or precursors inside a sealed container. This is heated to a moderately high temperature (usually lower than $250^{\circ} \mathrm{C}$ ) so that the internally-generated pressure can promote the growth of the material. Using this technique, it is also possible to grow materials on specific substrates by simply adding them to the reaction vessel. This is a very valuable strategy, as it can allow the surface modification of nanomaterials and the creation of hierarchical or "core-shell" structures.

Similarly to electrospinning, this technique also has a large number of parameters to choose from, both in terms of solution preparation and operational parameters such as temperature, pressure, and duration of heat treatment. These can have a very relevant impact on the growth of the materials, and can lead to the formation of different crystal phases or nanostructures, depending on the conditions used in the process.

\subsubsection{Thermal treatments}

Heat treatments are generally used in combination with the previously mentioned techniques as a fundamental step to obtain the desired materials. The fabrication of electrospun inorganic nanofibres, for instance, requires that a heat treatment is performed, so that the carrier polymer is removed; furthermore, inorganic materials often start from molecular precursors or salts, which need to be converted into the final product by heating the compounds, allowing it to be converted into the more thermodynamically stable material. Heat treatments can be either performed in air, using a muffle furnace, or under an inert atmosphere or vacuum using a tube furnace or a vacuum oven. These instruments are usually equipped with digital temperature controllers, which allow the heating and cooling processes to happen at a controlled rate; this can be useful to avoid thermal shock, 
which could lead to the disruption of both macroscopic and micro/nanoscopic structures. Furthermore, the target temperature and heating times can also be adjusted to obtain different results, such as different degrees of crystallinity, or different crystal phases altogether.

\subsection{Nanomaterials characterisation techniques}

\subsubsection{Electron microscopy}

Imaging is one of the most pivotal techniques for the characterisation nanomaterials. Electron microscopy is easily the most used technique for this purpose, as it allows to obtain information on the topography and morphology of materials at the nanoscale. This is made possible thanks to the shorter De Broglie wavelength of electrons compared to photons, which allow these techniques to achieve higher spatial resolutions compared to optical microscopy.

Scanning electron microscopy (SEM) and transmission electron microscopy (TEM) were both used in this $\mathrm{PhD}$ project. Although both techniques use an electron emitter as the illumination source, their working principle is fundamentally different: SEM imaging relies on the detection of electrons which are either reflected (back-scattered electrons) or re-emitted (secondary electrons) by the the specimen, and therefore mainly provides information on the surface morphology of the material. ${ }^{*}$ Furthermore, in order for the substrates to have enough contrast, the specimens need to possess a certain degree of conductivity; if the material is intrinsically not conductive, a thin layer of a conductive material such as carbon, gold, or platinum needs be deposited on the specimen.

TEM, on the other hand, relies on the detection of electrons transmitted through the sample. Because of this, it can can provide information on the internal composition of nanomaterials. This different working principle also allows for a larger lateral resolution $(<1 \AA)$ than SEM. Additionally, TEM can provide information on the crystal properties of the sample, both from high resolution images which can display the lattice fringes of the material, and from electron diffraction patterns.

*SEM images acquired with a back-scattered electron detector can also provide information on the elemental composition of the sample, as heavier elements can reflect electrons more efficiently, resulting in higher contrast. 
The higher resolution and additional features of TEM, however, come at the price of requiring a more complex and labour-intensive sample preparation, as specimens need to be thin enough (less than a few $\mu \mathrm{m}$ ) to allow the electron beam to penetrate the sample and reach the detector to form an image.

Both the instruments available at the facilities of this university are also equipped with energy-dispersive X-ray (EDX) detectors, which can provide spatiallyresolved information on the elemental composition of samples. Such information can be obtained in the form of bulk spectra, point or line analysis, or full twodimensional elemental concentration maps. The technique, however, is somewhat limited by low sensitivities for certain elements, overlapping X-ray emission peaks, lower spatial resolution, and sample-related issues such as drifting and degradation/melting from prolonged exposure to a focused electron beam.

\subsubsection{X-ray diffraction (XRD)}

Another fundamental characterisation technique for nanomaterials fabrication is $X$-ray diffraction. This technique relies on the property of materials to diffract incident $X$-rays at specific angles, based on the spatial arrangement of their atoms, according to Bragg's Law (Equation 3.1):

$$
n \lambda=2 d \sin \theta \quad \Longleftrightarrow \quad 2 d=\frac{n \lambda}{\sin \theta}
$$

In the above equation, $n$ is an integer number, $\lambda$ is the wavelength of the incident $\mathrm{X}$-rays, $d$ is the spacing between lattice planes of a given material, and $\theta$ is the incident angle of the X-ray beam. In materials science, powder XRD is routinely used to assess and quantify the type of crystal phases present in the sample. This is possible because each crystal structure has a specific set of diffraction peaks (also called a diffractogram), with consistent diffraction angles and relative intensities. Therefore, the crystal phase of a newly fabricated material can be determined by comparing its measured diffractogram with either a simulated profile, based on the predicted crystal structure, or an known diffractogram from a database such as the International Centre for Diffraction Data (ICDD). It is worth noting that, due to the way most commercial powder X-ray diffractometers are designed, diffraction data is usually reported in relation to the double of the Bragg angle, $2 \theta$. 
Another valuable piece of information that can be obtained from XRD is the degree of crystallinity and/or amorphousness of materials. This is possible because there is an inverse correlation between the line broadening of diffraction peaks and the mean size of crystal domains in the sample. A practical approximation of the physical principles behind this phenomenon can be found in the DebyeScherrer equation:

$$
\tau=\frac{K \lambda}{\beta \cos \theta}
$$

where $\tau$ is the mean size of crystalline domains, $K$ is a dimensionless number close to unity, related to the shape of the crystal domain, $\beta$ is the line breadth at half the maximum intensity in radians, and $\theta$ is the Bragg angle. Based on this equation, if a material is nanocrystalline or partially amorphous, very broad peaks should be expected to be observed in the diffractogram. Vice versa, if the material is highly crystalline, a series of sharp diffraction peaks should be observed.

\subsubsection{Fourier transform infrared (FT-IR) spectroscopy}

Similarly to the aforementioned characterisation techniques, FT-IR can be useful in materials characterisation to determine composition. This technique is generally used to study the absorption of electromagnetic radiation of a sample in the mid-infrared range (approximately between 4000 and $400 \mathrm{~cm}^{-1}$ ), which is associated with the excitation of vibrational energy states. This technique is especially useful when organic compounds are involved in the preparation of materials, as FT-IR can be used to determine the presence of organic species by looking for the signature vibrational transitions produced by the specific functional groups in the compound. In addition, many inorganic compounds also possess infrared-active vibrational modes, and therefore FT-IR spectroscopy can also serve as a further confirmation of the presence of a specific material in a sample.

For this project, a FT-IR spectrometer operating in attenuated total reflectance (ATR) was used: this method was particularly useful for electrospun nanofibre samples, as it allowed the material to be characterised directly, without any additional sample preparation. 


\section{Raman spectroscopy}

Raman spectroscopy is a technique which is also used to observe vibrational modes in materials. However, this method relies on a completely different principle from FT-IR: it is based on the inelastic scattering of a monochromatic light source by the analyte, shifting the energy state of the system to a higher (Stokes Raman scattering) or lower (Anti-Stokes Raman scattering) vibrational energy level. Because of this, different selection rules apply to this technique, and different vibrational modes are therefore detectable with this method, which are not observed in normal infrared spectroscopy.

One particularly useful application of Raman spectroscopy is the characterisation of carbon nanomaterials. These materials display two prominent Raman-active bands, one centred at $1350 \mathrm{~cm}^{-1}$ and one centred at $1580 \mathrm{~cm}^{-1}$, commonly referred to as the D and $\mathrm{G}$ band, respectively. The $\mathrm{D}$ band is associated with the presence of defects in graphitic carbon, such as $s p^{3}$-hybridised atoms and graphite edges. On the other hand, the $\mathrm{G}$ band is relative to $s p^{2}$-hybridised carbon. Therefore, by observing the shape and intensity ratio between these two bands, an estimate on the crystalline quality and defect content of the material can be made. ${ }^{15,16}$

\subsubsection{Thermogravimetric analysis (TGA)}

This technique consists in accurately measuring the change in mass of a sample placed inside a furnace with the temperature increasing at a steady rate. This provides information on the thermal stability of the sample at different temperatures, and can therefore have many applications in materials characterisation. For instance, it can be used to quantify the content of a certain component in material by examining the amount of mass lost at a temperature in which such component is known to decompose. Additionally, by understanding the temperatures at which the thermal decomposition steps take place in the material, one can tailor any heat treatments required to prepare such material in a way that optimises the selective elimination of materials that are not desired in the final product, such as the carrier polymer in inorganic electrospun nanofibres. Furthermore, the experiment can be run either under a flow of air or an inert gas, which provides additional information on the type of thermal decompositions taking place. 


\subsubsection{Brunauer-Emmett-Teller (BET) surface area measurement}

The BET method is the most commonly used technique to estimate the specific surface area of solids. The theory behind this method is based on an extension of the Langmuir theory, which models the physical adsorption of a gas as a molecular monolayer on the surface of the material at isothermal conditions. The measurement usually consists in the acquisition of an adsorption-desorption isotherm curve, which allows to measure the amount of absorbed gas via a continuous flow method. Because nitrogen is the most commonly used gas used, BET experiments are usually conducted at a temperature of $77 \mathrm{~K}$, which is the boiling point of $\mathrm{N}_{2}$. Although different gases can be used, they have a different boiling point and adsorption cross section, which will affect the specific surface area value measured. One important consideration is that this technique is dependent on many variables involving both the instrumental conditions (i.e. the type of gaseous adsorbate) and the characteristic of the sample (such as the type of pores present in the material): therefore, an absolute value of specific surface area cannot be defined, and the values obtained by this technique must only be considered as a reasonable estimate. ${ }^{17,18}$

\subsubsection{X-ray photoelectron spectroscopy (XPS)}

XPS is arguably one of the most important techniques for the characterisation of inorganic materials as it is used to identify the elemental composition and chemical state of a sample. XPS uses a monochromatic X-ray source to extract electrons from the atoms lying mainly in the superficial portion of a sample $(<10 \mathrm{~nm})$, through the photoelectric effect. The electrons escaping the material have kinetic energies which are directly related to their binding energies, corresponding to the electronic configuration of the relative atoms. More importantly, XPS allows to obtain information relative to the local bonding environment of a species: this is possible because factors such as oxidation state, neighbouring atoms, and bonding hybridisation can shift the binding energies of photoeletron peaks. Such chemical shifts can either be calculated theoretically, or determined experimentally using known materials for reference. ${ }^{19}$

The number of electrons detected in an XPS spectrum is directly related to the amount of the element present in the sample, accounting for a relative sensitivity 
factor (RSF), which is characteristic for every element. Only electrons that escape the sample can be effectively detected; emitted photoelectrons can be trapped in the material through a series of mechanisms, which are exponentially more prominent as the sample depth increases. Therefore, the signal detected in XPS is exponentially surface-weighted. However, most commercial instruments are also equipped with an ion gun, which can be used for etching the sample, allowing for depth profiling measurements.

Because accurate electron detection requires a long travel path before reaching the detector, XPS instruments are operated under ultra-high vacuum $\left(10^{-9}\right.$ torr $)$ to minimise signal loss due to collision of electrons with gas molecules. This also limits the use of the technique to non-volatile samples.

\subsection{Battery fabrication and testing}

\subsubsection{Electrode fabrication}

In order for nanomaterials to be used as electrodes in AIBs, two possible strategies are possible. If the material is obtained as a free-standing sheet, and its mechanical and electronic properties allow it, then it can be simply cut into an appropriate form factor and used directly in the device. On he other hand, if the material is not conductive enough, too fragile to be used as a self-standing electrode, or obtained in the form of a powder, then it must first be processed into a thin film to be used. The most commonly used procedure for this purpose consists of creating a slurry from the material, which is then cast as a film on a metal current collector substrate.

Preparation of the slurry usually starts with mixing the active material with a binder polymer, which is necessary to allow the film to adhere to the current collector. Because the binder polymer is generally a poor electric conductor, a small quantity of a conductivity enhancer such as carbon black is then also added to the mixture as a conductivity enhancer. N-methyl-2-pyrrolidone (NMP) is then added as a dispersant. Finally, mechanical and/or ultrasonic mixing is performed to produce the slurry. The slurry is then deposited onto the current collector; doc-

tor blading is usually the technique of choice for this step, thanks to its simplicity, ease of use, and scalability. ${ }^{20}$ Finally, a mild heat treatment under vacuum is used 
to evaporate the dispersant and fixate the film on the current collector. An optional step is to use a rolling press or a similar instrument to apply pressure on the electrode, which improves adhesion of the film to the current collector, and compacts the active layer, improving the contact between the active material particles and enhancing the overall conductivity of the electrode. After this step, the fabrication process is finished and the electrode is ready to be used. There are many parameters that can be varied in this process, and the final result can be greatly affected by the conditions adopted in the fabrication phase. It is therefore important to use special care in optimising the conditions of this process.

\subsubsection{Prototype battery devices}

Many different cell designs can be used to build a prototype battery for testing the performance of electrodes and electrolytes. For this PhD project, "Swagelok-type" cells were the design of choice. This type of cell takes its name from the manufacturing brand of pipe fittings, which are used as a casing for the cell. In a typical Swagelok-type cell design, a three-piece, screw-capped straight union pipe fitting is used as the body of the device and two metal rods are fitted at the opposite ends of the joint to serve as terminals for the battery. The device is assembled by cutting the active components to fit in the inner diameter of the pipe fitting, and subsequently placing them inside the cell by stacking the cathode, separator (which is soaked in the electrolyte), and anode inside the Swagelok fitting. The metal rods are then inserted in the fitting, and screwed in place (Figure 3.2).

For AIBs, the assembly process usually takes place inside of a nitrogen- or argonfilled glovebox, to avoid contamination from atmospheric oxygen and water. Two rubber O-rings inside the screw caps of the pipe fitting ensure that the internal components of the device are isolated from the outside atmosphere and no electrolyte can leak out of the device. If necessary, Parafilm can also be wrapped around the casing for additional protection. After this step, the cell is taken outside the glovebox, and is ready to be tested.

The modular design of this cell type allows to easily assemble and test devices without the need for sophisticated instrumentation. Furthermore, the devices can be easily disassembled, and the active components can be reclaimed and used for 

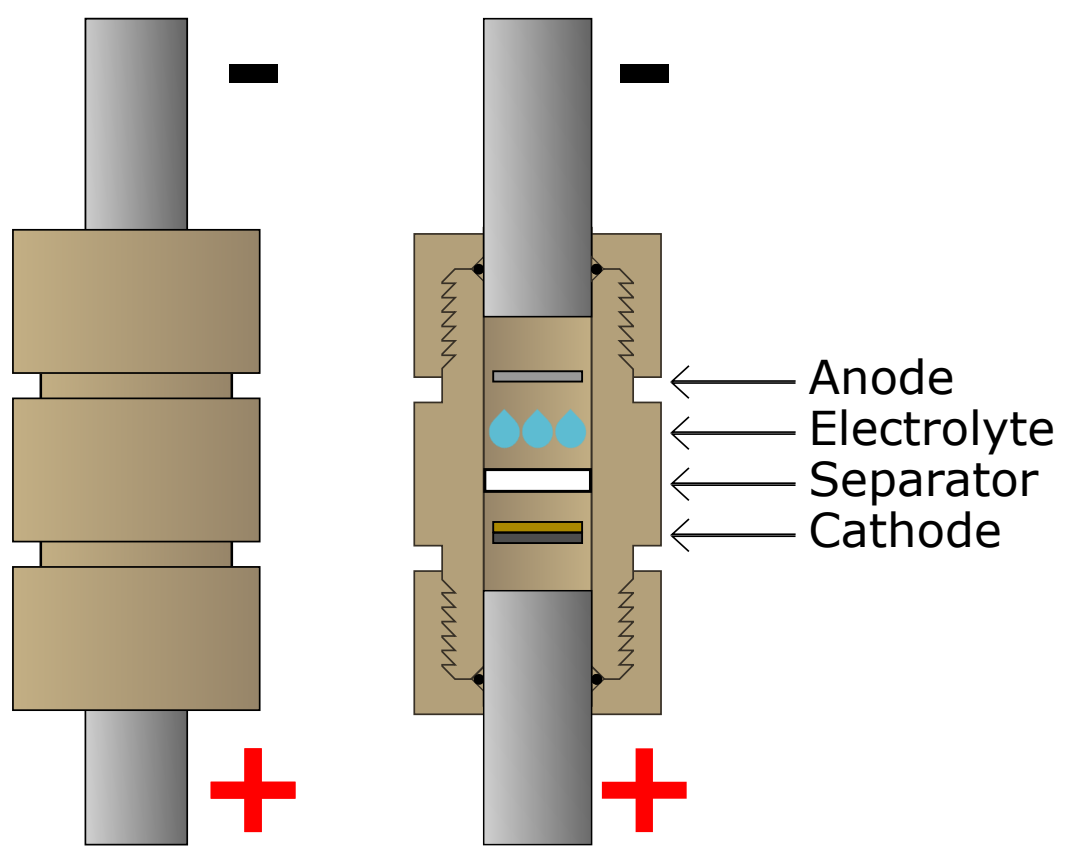

FIGURE 3.2: Schematic illustrating the design and internal construction of a prototype Swagelok-type cell.

ex situ analysis. Finally, unlike other designs such as pouch cells or coin cells, ${ }^{\dagger}$ the casing can be washed and reused indefinitely. For the experiments performed in this thesis, a custom-built three-piece fitting, with a design similar to the commercially available Swagelok union joints, was machined from polyether ether ketone (PEEK) by the mechanical workshop at Victoria University of Wellington. This material was chosen thanks to its chemical inertness and mechanical properties, which allowed it to be fabricated using a lathe. The inner diameter of the casing was chosen to be $12 \mathrm{~mm}$, so that the construction of devices would require smaller quantities of active materials and electrolytes. For the current collector, stainless steel rods were initially chosen. However, experimental evidence revealed that this was not an appropriate choice, as the material tended to react with the electrolyte, thus affecting the performance of the device and invalidating test results. Some literature evidence also confirms that stainless steel is not an adequate current collector. ${ }^{21}$ Therefore, molybdenum rods were instead used as the terminals, as the metal shows no reactivity towards the electrolyte. ${ }^{22}$

${ }^{\dagger}$ These cell designs can generally provide more reliable performance, but require more elaborate preparation methods and dedicated instrumentation. 


\subsubsection{Electrochemical testing}

Once the devices are assembled, a series of electrochemical tests can be performed to assess the performance of the novel materials and electrolytes. It is worth noting that, based on the component of the device that is investigated, the experiment needs to be designed in a way that the result of the tests can be attributed exclusively to such component. For instance, if a new electrode material is fabricated, and its behaviour in the battery electrochemistry wants to be determined, then the remaining components of the device should be chosen among known "benchmark" materials that can afford reliable and easily reproducible results. This way, the number of variables is minimised, and the causal relationships between the test results and the properties of the new material are easier to determine. Furthermore, by using a benchmark material, the results are standardised and easier to compare with other literature reports. For AIBs, it is easy to argue that such materials should be high-purity $\mathrm{Al}$ foil as the anode, graphite (natural or pyrolytic) as the cathode, and a 1:1.3 molar ratio mixture of [EMIm] $\mathrm{Cl}$ and $\mathrm{AlCl}_{3}$ as the electrolyte. In addition, if the performance of a new cathode material is to be tested and quantified, then the device must be constructed in a way such that the quantities of anode and electrolyte are in a large excess, so that the cathode mass is the limiting factor, or "bottleneck" in the performance of the device.

\section{Cyclic voltammetry}

If a novel cathode or electrolyte is fabricated, it is worth studying its electrochemistry by performing a preliminary cyclic voltammetry $(\mathrm{CV})$ experiment. This type of measurement consists of applying a series of sequential increasing and decreasing potential ramps to an electrode and measuring the changes in current. The resulting profile (also known as a cyclic voltammogram) can give information on the redox processes taking place in the device. This is a particularly useful experiment for battery testing, as it can provide some initial information on the presence of reversible electrochemical reactions, which are responsible for the energy storage mechanism of the battery. Furthermore, the presence of capacitive or pseudocapacitive behaviour in the device can be also inferred by observing the overall profile of the voltammogram, and other in-depth experiments are also possible to determine and quantify their contribution to the overall battery chemistry. ${ }^{23}$ 
In a typical CV experiment, two types of current can be observed: faradaic and capacitive current. Faradaic current is related to redox reactions taking at the electrode when a certain potential is applied, and figures as a peak in the voltammogram: an oxidation reaction will give rise to a positive peak, and a reduction will correspond to a negative peak (Figure 3.3 (left)). On the other hand, capacitive current is caused by the adsorption of charged species on the electrode, and the formation of a capacitive double layer; this current manifests itself as a hysteretic curve in the voltammogram (Figure 3.3 (right)). Although the capacitive contribution to the current is often negligible, if the electrode material has a high surface area then such contribution can become more prominent.
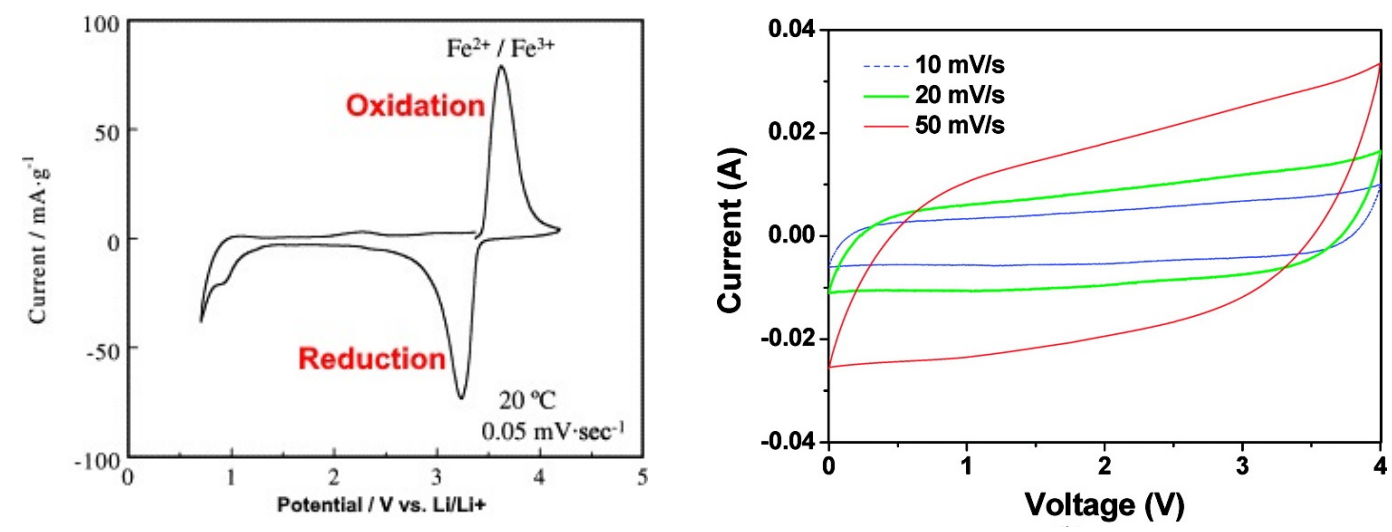

FIGURE 3.3: Cyclical voltammograms showing examples of faradaic (left) and capacitive (right) currents: a $\mathrm{LiFePO}_{4}$ cathode for LIBs (left) from Takahashi $e t$ $a l .{ }^{24}$ and a graphene-based supercapacitor (right) from Liu et al. (Copyright 2010 American Chemical Society). ${ }^{25}$ Adapted with permission from the publications.

Although cyclic voltammetries can be performed on a Swagelok-type cell using a two-electrode configuration, the ideal setup for this experiment is a threeelectrode flooded cell. In this configuration, a larger volume of electrolyte is present, and the electrochemical cell is wired in such a way that the potential is applied to a working electrode (WE) against the reference electrode (RE), but the current flows between the working and counter electrodes (CE). The advantage of using a reference electrode to measure the potential is that it provides a more accurate and reliable reading of the voltage, as an electric current flowing through the electrode would otherwise cause the potential to drift over time. ${ }^{26}$ Furthermore, the large volume of electrolyte allows the dynamics of the electrochemical processes to be controlled by diffusion, which is the ideal regime in which the theory behind this type of experiment is formulated. 
Cyclic voltammograms are usually reported either as the measured current versus the applied voltage or as current density versus voltage, which is found by dividing the measured current by the surface area of the working electrode. For the voltammograms reported in this PhD thesis, it is worth noting that only the macroscopic surface of the electrode was considered to calculate current densities, and specific surface area measurements such as the Brunauer-Emmett-Teller gas adsorption-desorption method are not taken into consideration for such calculation.

\section{Galvanostatic charge-discharge}

The most important test for battery performance is easily galvanostatic cycling. This test consists of applying a constant current to the cell and monitoring the change of voltage over time (also known as a chronopotentiogram). ${ }^{\ddagger}$ If a positive current is applied, the step is considered as a "charging" phase, and the voltage is expected to gradually increase. Vice versa, if a negative (or "discharging") current is applied, the voltage is expected to decrease. As soon as a pre-determined voltage is reached, the current is reversed, and the next step begins. A combination of a charging and a discharging step constitute a full cycle; usually, a series of cycles are performed to determine the performance variation over the subsequent cycles. Depending on the assumed electrochemical reactions taking place in the device, a newly assembled device may be in either a discharged or charged state. Therefore, based on the initial state of the devices, the cycling protocols should be started with either a charging or a discharging step accordingly.

Typically, galvanostatic cycles are conducted between a predetermined upper and lower voltage limit (also called "cut-off" voltages). This window is usually selected in a way which ensures that only the desirable redox processes are taking place in the device, and unwanted irreversible reactions are avoided. This maximises the efficiency of the device and avoids any damage due to unwanted reactions. Although the upper and lower voltage limits can be determined by trial and error or taken from literature values, the most accurate way of determining the ideal

\footnotetext{
¥The phrasing "applying a constant current" is somewhat misleading, as the experiment technically consists in modulating the applied potential so that the current is maintained constant. For the sake of simplicity, this terminology will be briefly used in this section.
} 
voltage range of the device is by performing cyclic voltammetry experiments: irreversible processes can be immediately found by observing whether any oxidation peak does not have a corresponding reduction peak at similar voltage, or vice versa. Furthermore, the stability window of the electrolyte can also be determined by a CV experiment with "inert" WE and CE. For instance, glassy carbon does not exhibit any cathodic behaviour for AIBs, so when it is used as a WE, no faradaic current can be seen. Activity is only observed at extreme potentials, which mark the decomposition of the electrolyte. For the "benchmark" $\mathrm{AlCl}_{3} /[\mathrm{EMIm}] \mathrm{Cl} \mathrm{AIB}$ electrolyte, the useful/nondestructive voltage window is usually considered to be between 0 and $2.5 \mathrm{~V}$ vs Al/ $\mathrm{Al}^{3+}$.

A great deal of information can be obtained from such galvanostatic cycling. First of all, the practical specific capacity of an electrode material can be measured for a charging and discharging step. This is done by multiplying the step time by the applied current, and dividing by the mass of active material in the electrode, according to the following equation:

$$
\mathrm{C}_{\text {step }}=\frac{\mathrm{t}_{\text {step }} \times \mathrm{i}_{\text {step }}}{\mathrm{m}_{\text {electrode }}}
$$

where $C_{\text {step }}$ is the specific capacity of a given step, $t_{\text {step }}$ is the charging or discharging time of that step, $i_{\text {step }}$ is the applied current in that step, and $m_{\text {electrode }}$ is the mass of active material in the electrode. As previously explained, this formula relies on the assumption that the remaining components are present in high excess compared to the electrode, therefore not constituting a "bottleneck" to the overall performance of the device.

Furthermore, if the device works correctly and redox reactions are taking place, the trend of the galvanostatic profiles should display the presence of plateaus in both the charging and discharging step, indicating the presence of faradaic processes. The voltage at which these plateaus appear should roughly reflect the voltage at which the corresponding peaks are observed in the cyclic voltammetry, and therefore serve as a good way of confirming the behaviour of the cell.

By calculating the ratio between the charging and discharging capacities within the same cycle, the coulombic efficiency of that cycle is obtained. This value is commonly reported as a percentage value, therefore the ratio is usually multiplied by 100 . This is an important metric, as it provides an indication of the efficiency of 
the process; furthermore, if this value deviates largely from $100 \%$, it could be an indication of the presence of unwanted side reactions or irreversible electrochemical processes in the device.

By integrating the voltage profile over the specific capacity, the specific energy of the electrode for a given step can also be obtained. Alternatively, this value can also be estimated by multiplying the specific capacity by the average voltage of the step.

In a typical experiment, multiple current rates are used, with several cycles performed at each rate. This is useful to study the capacity retention of the device at higher current rates; in addition, the capacity retention can be monitored over a large number of cycles, which gives indication on the lifetime performance of the device.

\section{Bibliography}

${ }^{1}$ A. S. Aricò, P. Bruce, B. Scrosati, J.-M. Tarascon, and W. van Schalkwijk, "Nanostructured materials for advanced energy conversion and storage devices," Nature Materials, vol. 4, pp. 366-377, May 2005.

${ }^{2}$ T. J. Macdonald, D. D. Tune, M. R. Dewi, C. T. Gibson, J. G. Shapter, and T. Nann, “A TiO2 Nanofiber-Carbon Nanotube-Composite Photoanode for Improved Efficiency in Dye-Sensitized Solar Cells," ChemSusChem, vol. 8, pp. 3396-3400, Oct. 2015.

${ }^{3} \mathrm{D}$. Li and Y. Xia, “Electrospinning of Nanofibers: Reinventing the Wheel?," Advanced Materials, vol. 16, pp. 1151-1170, July 2004.

${ }^{4}$ P. Viswanathamurthi, N. Bhattarai, H. Y. Kim, and D. R. Lee, "Vanadium pentoxide nanofibers by electrospinning," Scripta Materialia, vol. 49, pp. 577-581, Sept. 2003.

${ }^{5}$ S. K. Nataraj, K. S. Yang, and T. M. Aminabhavi, "Polyacrylonitrile-based nanofibers-A stateof-the-art review," Progress in Polymer Science, vol. 37, pp. 487-513, Mar. 2012.

${ }^{6} \mathrm{H}$. Wu, R. Zhang, X. Liu, D. Lin, and W. Pan, "Electrospinning of Fe, Co, and Ni Nanofibers: Synthesis, Assembly, and Magnetic Properties," Chemistry of Materials, vol. 19, pp. 3506-3511, July 2007.

${ }^{7}$ N. C. Hildebrandt, J. Soldat, and R. Marschall, “Layered Perovskite Nanofibers via Electrospinning for Overall Water Splitting," Small, vol. 11, pp. 2051-2057, May 2015.

${ }^{8}$ L. Persano, A. Camposeo, C. Tekmen, and D. Pisignano, “Industrial Upscaling of Electrospinning and Applications of Polymer Nanofibers: A Review," Macromolecular Materials and Engineering, vol. 298, pp. 504-520, May 2013.

${ }^{9}$ H. Jiang, Y. Hu, Y. Li, P. Zhao, K. Zhu, and W. Chen, "A facile technique to prepare biodegradable coaxial electrospun nanofibers for controlled release of bioactive agents," Journal of Controlled Release, vol. 108, pp. 237-243, Nov. 2005. 
${ }^{10}$ D. Li and Y. Xia, "Direct Fabrication of Composite and Ceramic Hollow Nanofibers by Electrospinning," Nano Letters, vol. 4, pp. 933-938, May 2004.

${ }^{11}$ X. Lu, C. Wang, and Y. Wei, "One-Dimensional Composite Nanomaterials: Synthesis by Electrospinning and Their Applications," Small, vol. 5, pp. 2349-2370, Nov. 2009.

12 T. Zhai, H. Liu, H. Li, X. Fang, M. Liao, L. Li, H. Zhou, Y. Koide, Y. Bando, and D. Golberg, "Centimeter-Long V2O5 Nanowires: From Synthesis to Field-Emission, Electrochemical, Electrical Transport, and Photoconductive Properties," Advanced Materials, vol. 22, pp. 2547-2552, June 2010.

${ }^{13}$ M. R. Parida, C. Vijayan, C. S. Rout, C. S. S. Sandeep, R. Philip, and P. C. Deshmukh, "Room Temperature Ferromagnetism and Optical Limiting in V2O5 Nanoflowers Synthesized by a Novel Method," The Journal of Physical Chemistry C, vol. 115, pp. 112-117, Jan. 2011.

${ }^{14}$ J. Mu, J. Wang, J. Hao, P. Cao, S. Zhao, W. Zeng, B. Miao, and S. Xu, "Hydrothermal synthesis and electrochemical properties of V2O5 nanomaterials with different dimensions," Ceramics International, vol. 41, pp. 12626-12632, Dec. 2015.

${ }^{15}$ A. C. Ferrari, "Raman spectroscopy of graphene and graphite: Disorder, electron-phonon coupling, doping and nonadiabatic effects," Exploring graphene, vol. 143, pp. 47-57, July 2007.

${ }^{16}$ L. Malard, M. Pimenta, G. Dresselhaus, and M. Dresselhaus, "Raman spectroscopy in graphene," Physics Reports, vol. 473, pp. 51-87, Apr. 2009.

${ }^{17}$ S. Brunauer, P. H. Emmett, and E. Teller, "Adsorption of Gases in Multimolecular Layers," Journal of the American Chemical Society, vol. 60, pp. 309-319, Feb. 1938.

${ }^{18}$ D. A. H. Hanaor, M. Ghadiri, W. Chrzanowski, and Y. Gan, "Scalable Surface Area Characterization by Electrokinetic Analysis of Complex Anion Adsorption," Langmuir, vol. 30, pp. 1514315152, Dec. 2014.

${ }^{19}$ J. F. Moulder, W. F. Stickle, P. E. Sobol, and K. D. Bomben, Handbook of X Ray Photoelectron Spectroscopy. Eden Prairie, Minn: Physical Electronics, Feb. 1993.

${ }^{20}$ A. Berni, M. Mennig, and H. Schmidt, "Doctor Blade," in Sol-Gel Technologies for Glass Producers and Users (M. A. Aegerter and M. Mennig, eds.), pp. 89-92, Boston, MA: Springer US, 2004.

${ }^{21}$ L. D. Reed and E. Menke, "The Roles of V2O5 and Stainless Steel in Rechargeable Al-Ion Batteries," Journal of the Electrochemical Society, vol. 160, no. 6, pp. A915-A917, 2013.

${ }^{22}$ Y. Oh, G. Lee, and Y. Tak, "Stability of Metallic Current Collectors in Acidic Ionic Liquid for Rechargeable Aluminum-Ion Batteries," ChemElectroChem, vol. 5, pp. 3348-3352, Nov. 2018.

${ }^{23}$ H.-S. Kim, J. B. Cook, H. Lin, J. S. Ko, S. H. Tolbert, V. Ozolins, and B. Dunn, “Oxygen vacancies enhance pseudocapacitive charge storage properties of $\mathrm{MoO}(3-\mathrm{x})$, " Nature Materials, vol. 16, pp. 454-460, Apr. 2017.

${ }^{24}$ M. Takahashi, S.-i. Tobishima, K. Takei, and Y. Sakurai, "Reaction behavior of LiFePO4 as a cathode material for rechargeable lithium batteries," Solid State Ionics, vol. 148, pp. 283-289, June 2002.

${ }^{25}$ C. Liu, Z. Yu, D. Neff, A. Zhamu, and B. Z. Jang, "Graphene-Based Supercapacitor with an Ultrahigh Energy Density," Nano Letters, vol. 10, pp. 4863-4868, Dec. 2010.

${ }^{26}$ N. Elgrishi, K. J. Rountree, B. D. McCarthy, E. S. Rountree, T. T. Eisenhart, and J. L. Dempsey, "A Practical Beginner's Guide to Cyclic Voltammetry," Journal of Chemical Education, vol. 95, pp. 197-206, Feb. 2018. 
Chapter 4

Cathode materials: $\mathrm{V}_{2} \mathrm{O}_{5}$ nanofibres 



\subsection{Preface}

The first item undertaken as part of this project was studying vanadium oxide nanofibres (NFs) as a cathode material for AIBs.

Vanadium $(\mathrm{V})$ oxide $\left(\mathrm{V}_{2} \mathrm{O}_{5}\right)$ is a well-known inorganic material with many applications in various fields of research, including catalysis and optics. ${ }^{1,2}$ One of the most prominent applications, however, is its use as an electrode material for Li-ion and other emerging battery technologies. ${ }^{3-5}$ Notably, the first paper presenting a fully functional AIB, published by Jayaprakash et al., ${ }^{6}$ used a $\mathrm{V}_{2} \mathrm{O}_{5}$ nanowirebased cathode. The authors claimed a remarkably high specific capacity of about $300 \mathrm{mAh} \mathrm{g}^{-1}$ for this cathode, and a lifespan of 20 cycles. This paper was the main inspiration for undertaking this project, as the battery performance reported in this paper showed very promising results with much room for improvement. It was initially hypothesised to achieve improved performance by using a different type of $\mathrm{V}_{2} \mathrm{O}_{5}$ nanomaterial as cathode. Electrospun $\mathrm{V}_{2} \mathrm{O}_{5}$ NFs were chosen to be used as the alternative cathode material candidate. This choice was mainly due to the relatively low complexity of the fabrication process (see also Section 3.2.1), and to the pre-existing expertise of the technique within our research group. ${ }^{7}$ The material itself is not novel: there are, in fact, many reports on the preparation of $\mathrm{V}_{2} \mathrm{O}_{5}$ NFs in the literature, often geared towards energy-related applications. ${ }^{8-14}$ At the time this project was designed, however, no publication describing the use of this material in AIBs was present. We therefore decided to undertake this research path and attempt using $\mathrm{V}_{2} \mathrm{O}_{5} \mathrm{NFs}$ as a cathode for AIBs.

\subsection{Nanofibre fabrication methods}

\subsubsection{Unsuccessful attempts}

The synthesis of $\mathrm{V}_{2} \mathrm{O}_{5}$ NFs by electrospinning generally involves preparing a solution containing a carrier polymer and a vanadium precursor. The solution is then electrospun to obtain hybrid vanadium/polymeric nanofibres. A calcination step to a temperature of $400-500{ }^{\circ} \mathrm{C}$ in air is then used to decompose and remove the carrier polymer, and convert the fibres to pure vanadium oxide. Based on the large amount of published works on the subject, this step was initially expected 
to be trivial. Unfortunately, most attempts at replicating previous literature processes have been either unsuccessful or underwhelming.

The first procedure that was attempted claimed these fibres could be produced from a solution of vanadyl oxalate and polyvinylpyrrolidone (PVP) in a $\mathrm{H}_{2} \mathrm{O} / \mathrm{N}$, N'-dimethylformamide (DMF) mixture as the precursor solution. ${ }^{14}$ Following the procedure, the solution was readily prepared and electrospun with the same parameters used in the publication, but the resulting material had a vastly different morphology from the one reported in the publication. SEM imaging revealed that only very thin nanofibres with numerous bead-shaped defects were obtained, as opposed to the expected smooth, 200-300 nm diameters fibres claimed by the authors of the paper. Furthermore, after performing the same heat treatment for the calcination step used by the authors, the fibrous morphology of the material was completely lost, leading to a bulk powder (Figure 4.1).
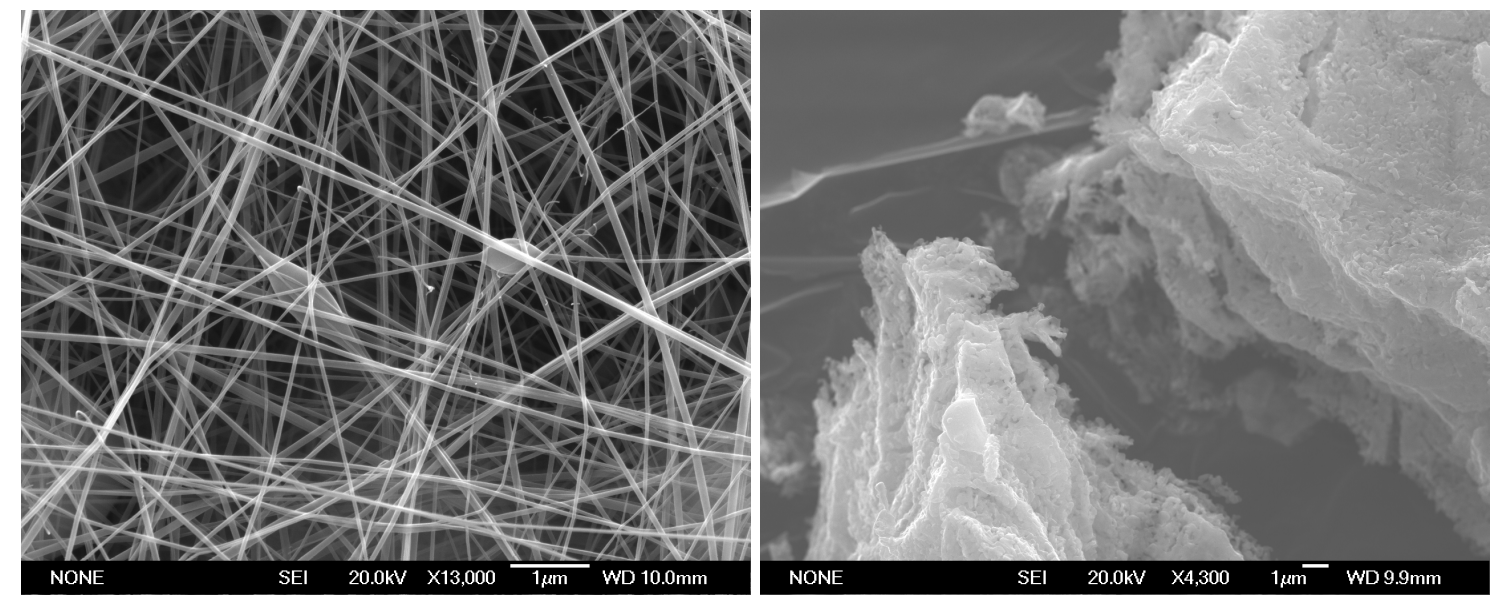

FIGURE 4.1: (Left) SEM micrograph of the precursor nanofibres prepared according to the procedure described by Li et al. ${ }^{14}$ (Right) SEM micrograph of the same material after undergoing the reported calcination step.

Similarly underwhelming results were also obtained when trying to replicate other publications, which used alternative procedures for the fabrication of $\mathrm{V}_{2} \mathrm{O}_{5}$ NFs. ${ }^{8,9,12}$ The literature on the topic describes a large variety of methods, using different precursor solutions, electrospinning parameters, and calcination methods. The main difference between the initial and subsequent protocols that were followed was the use of different vanadium precursors for the electrospinning 
solution: compounds such as vanadyl acetylacetonate $\left(\mathrm{VO}(\mathrm{acac}){ }_{2}\right){ }_{,}^{8}$ peroxovanadates, ${ }^{9}$ and vanadium oxytriethoxide ${ }^{12}$ were used in these new attempts. Unfortunately, no valid nanomaterial could be obtained from these procedures (see also Figure A.1).

Because following literature precisely did not yield acceptable results, a certain degree of parameter tweaking and optimisation was attempted. Solution concentrations and electrospinning parameters were modified on a trial-and-error basis, leading to some improvements in the resulting material morphology. Using this method, a somewhat acceptable result was eventually obtained after modifying the synthetic procedure used in a publication by Wang et al. ${ }^{11}$ Briefly, $\mathrm{VO}(\mathrm{acac})_{2}$ and PVP were dissolved in a mixture of ethanol and DMF to form a viscous precursor solution, which was electrospun using the same conditions reported on the aforementioned paper. Smooth, defect-free precursor nanofibres, with diameters ranging between 200 and $500 \mathrm{~nm}$, were obtained. After the calcination process, however, the material became extremely fragile and brittle; SEM images confirmed that although the fibrous morphology was mostly retained, the process converted the smooth nanofibres into hollow tubular structures that were far shorter in length (Figure 4.2). Despite looking notably better than previous attempts, this material was deemed not ideal for the desired application, as it appeared that much of the fibrous structure was lost in the calcination process, and it would likely disintegrate completely in the subsequent electrode fabrication processes required to use the material in batteries.
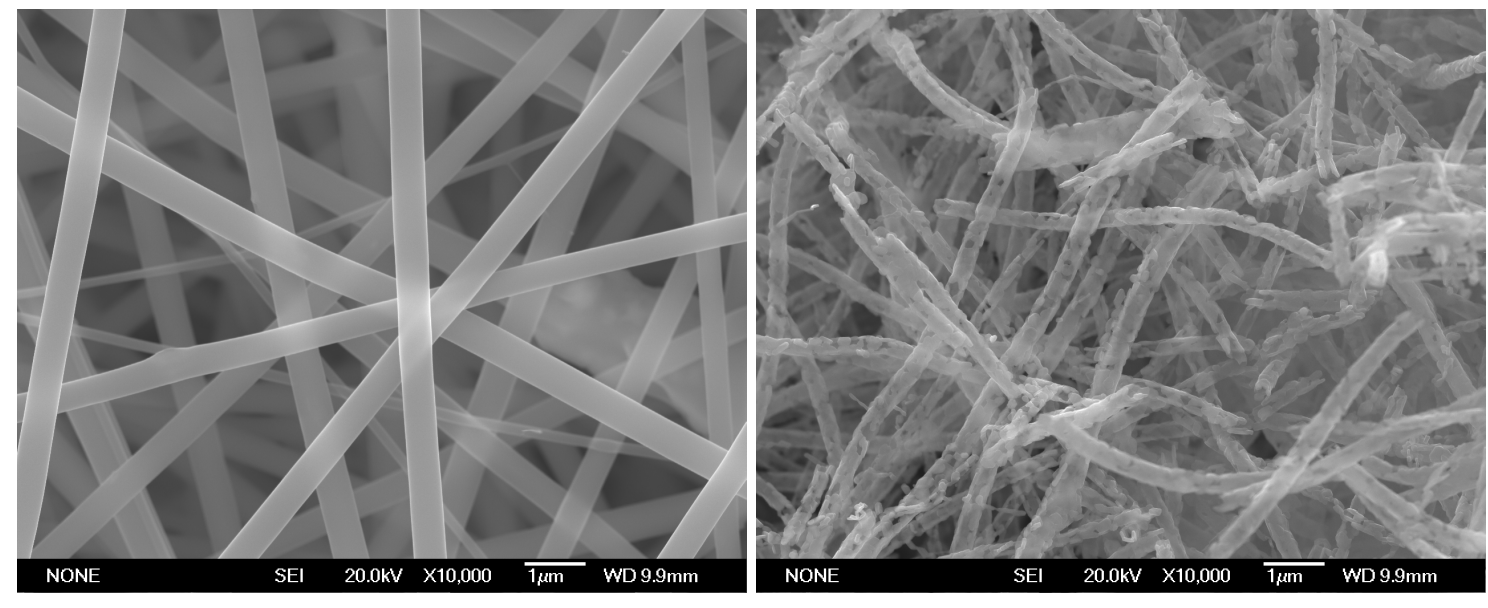

FIGURE 4.2: (Left) SEM micrograph of the precursor nanofibres prepared by adapting the procedure described by Wang et al. ${ }^{11}$ (Right) SEM micrograph of nanofibres after undergoing the reported calcination step. 
The failure to obtain acceptable results from literature procedures begs the question of why it is so difficult to replicate pre-established protocols for the fabrication $\mathrm{V}_{2} \mathrm{O}_{5}$ NFs. This was believed to be mainly ascribed to two factors:

1. There is a certain variance within the results due to the different electrospinning setups used by different groups. Such setups are often house-built and could therefore yield less reproducible results.

2. The vast majority of publications describing the preparation of electrospun $\mathrm{V}_{2} \mathrm{O}_{5} \mathrm{NFs}$ do not report the environmental conditions (i.e. temperature and humidity) under which the electrospinning process was performed. This can have a definite impact on the final morphology of the nanofibres, as ambient temperature and humidity affect the dielectric constant of air and the rate of solvent evaporation, considerably modifying the conditions, and can therefore resulting in a material with a vastly different morphology. ${ }^{15}$

\subsubsection{A novel fabrication method}

The disappointing results obtained by trying to reproduce literature procedures led to the decision of developing an entirely new protocol to fabricate $\mathrm{V}_{2} \mathrm{O}_{5} \mathrm{NFs}$ from scratch. In order to do so, all the parameters for the process needed to be re-evaluated, starting from the precursor solution.

The most important decision to be made for the development of a new protocol for the fabrication of $\mathrm{V}_{2} \mathrm{O}_{5}$ NFs was arguably the choice of vanadium precursor. Such decision was based on the results of the previous experiments: when using vanadium oxytriethoxide as the precursor in the electrospinning solution, ${ }^{12}$ the obtained material displayed a reasonably good fibrous morphology that could also be retained after the calcination step (see also Figures A.1 and A.2). However, the electrospinning phase itself proved to be quite challenging, as the nozzle tended get clogged shortly after starting to extrude the solution, interrupting the process. This was most likely due to the high reactivity of the vanadium oxyalkoxide precursor with atmospheric moisture. As soon as the precursor solution exited the needle and came into contact with the atmosphere, the vanadium oxytriethoxide would immediately react with the water molecules in the air to undergo a hydrolysis reaction and forming a gel which gradually hardened. The remaining solution would therefore get blocked by this gel and could no longer 
flow through the nozzle, bringing the process to a halt. Based on this observation, a possible solution solution would be to to find a vanadium-containing precursor with similar characteristics, but lower reactivity. It is known that one of the best strategies to slow down the sol-gel process of metal alkoxides is to use compounds with longer, more sterically encumbered alkoxide groups. ${ }^{16}$ Therefore, vanadium oxytriisopropoxide (VOTIP) was chosen to use as the inorganic precursor. This proved to be a good decision, as VOTIP-containing precursor solutions could be easily electrospun continuously, without the undesirable gel formation.

Following the selection of an appropriate vanadium precursor, the remaining parameters of the synthetic protocol were notably less complex to decide: only a few iterative steps in the optimisation of the solution concentration and the instrumental conditions were required, and the protocol for the preparation of $\mathrm{V}_{2} \mathrm{O}_{5}$ NFs was finally developed. The final calcined product appears to be composed of individual crystal grains of about $50-80 \mathrm{~nm}$ in size, fused together to form long fibres, with a high aspect ratio and an oblong, ribbon-like cross section. Diameters of the nanofibres ranged roughly between 500 and $800 \mathrm{~nm}$ (Figures 4.3 and A.3).
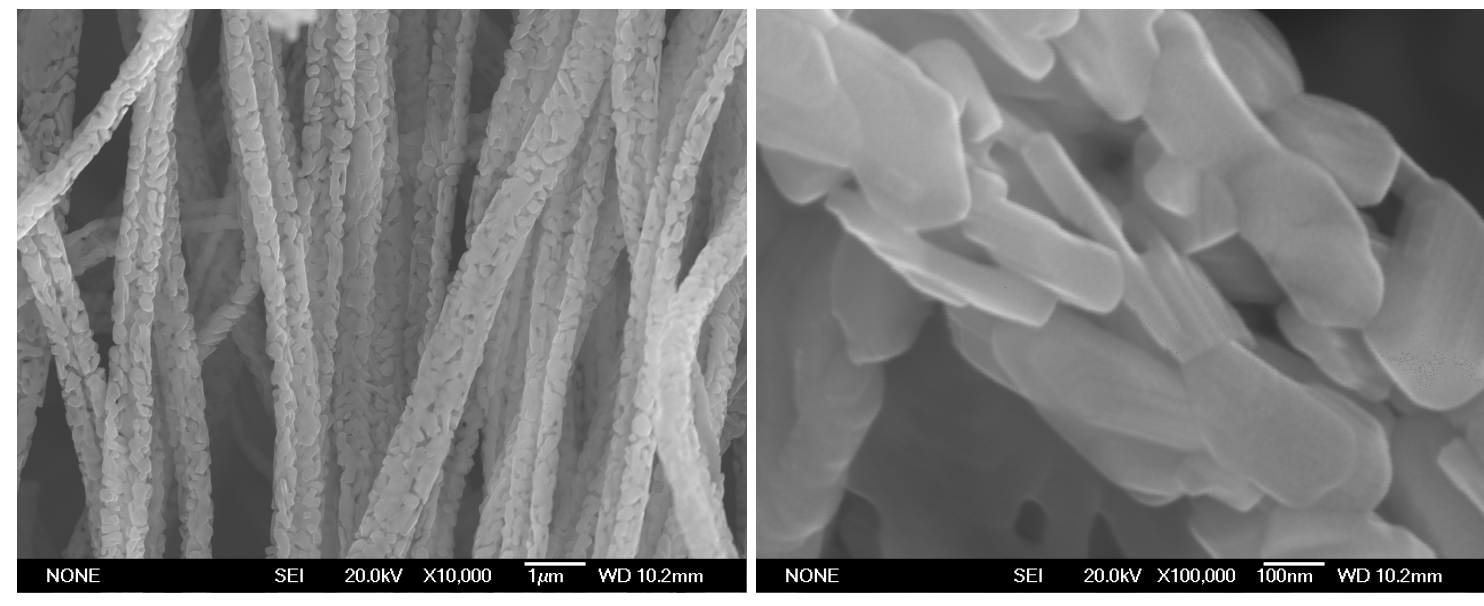

FIGURE 4.3: (Left) SEM micrograph of the $\mathrm{V}_{2} \mathrm{O}_{5}$ NFs obtained with the novel fabrication method. (Right) High-magnification SEM micrograph displaying the hierarchical nanostructure of the fibres.

In order to confirm the complete removal of the carrier polymer and assess the crystalline phase of the product, FT-IR spectroscopy and XRD were performed on the material. The FT-IR spectra show that the polymer is clearly present in the nanofibres prior to the calcination treatment, as it can be inferred by the presence of a large quantity of peaks in the spectrum that are ascribable to the vibrational 


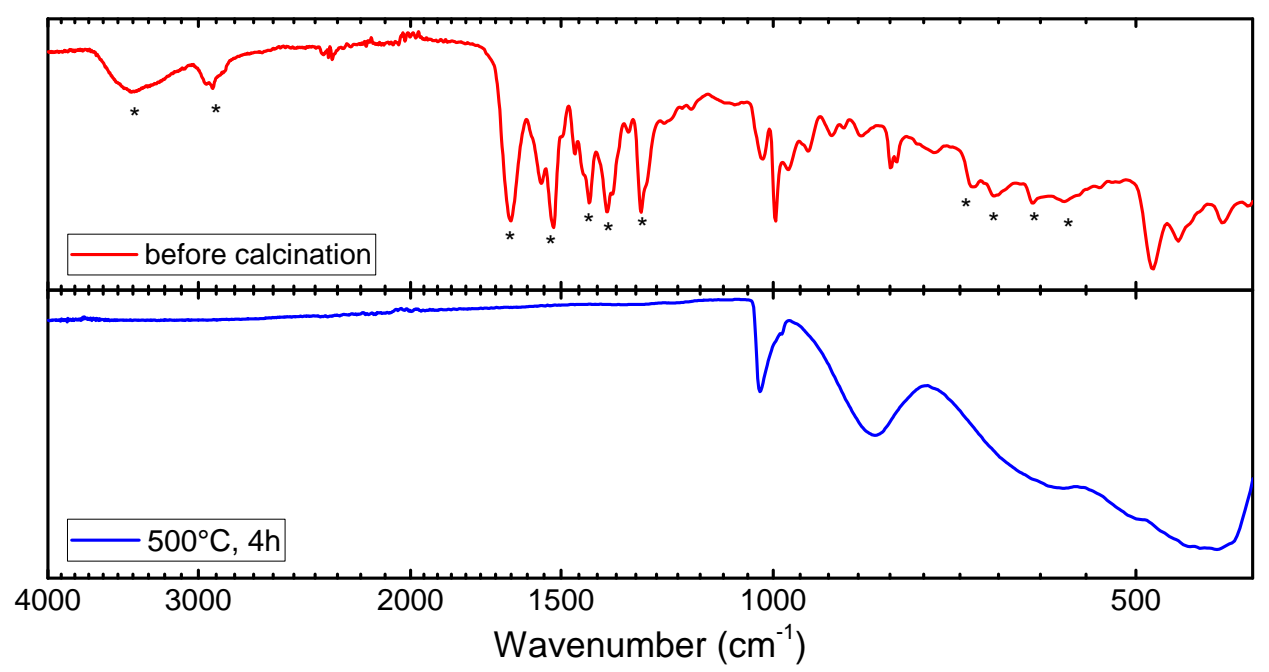

FIGURE 4.4: FT-IR spectra of the $\mathrm{V}_{2} \mathrm{O}_{5}$ nanofibres before (top) and after (bottom) the calcination step. The peaks marked with * are imputable to PVP. The remaining peaks in the top spectrum are likely ascribable to the partially hydrolysed vanadium precursor. ${ }^{17}$

modes of PVP. However, after the heat treatment, no peaks relative to the carrier polymer can be identified, indicating its complete removal from the nanofibres (Figure 4.4).

X-ray diffraction was also performed on the material. The diffractogram of the nanofibres presents a series of peaks that can be unequivocally attributed to orthorhombic vanadium (V) oxide; the peaks are well matched with a diffractogram from the International Centre for Diffraction Data (ICDD) database for $\mathrm{V}_{2} \mathrm{O}_{5}$, confirming that the desired crystal phase was obtained with high purity. Furthermore, the sharp diffraction peaks indicate the high crystallinity of the product (Figure 4.5).

Based on the morphology, crystallinity, and purity of the obtained $\mathrm{V}_{2} \mathrm{O}_{5} \mathrm{NFs}$, this material was deemed as satisfying for its intended purpose. Therefore the following step of the project, consisting of the fabrication of devices using $\mathrm{V}_{2} \mathrm{O}_{5}$ NFs as cathode, was undertaken. 


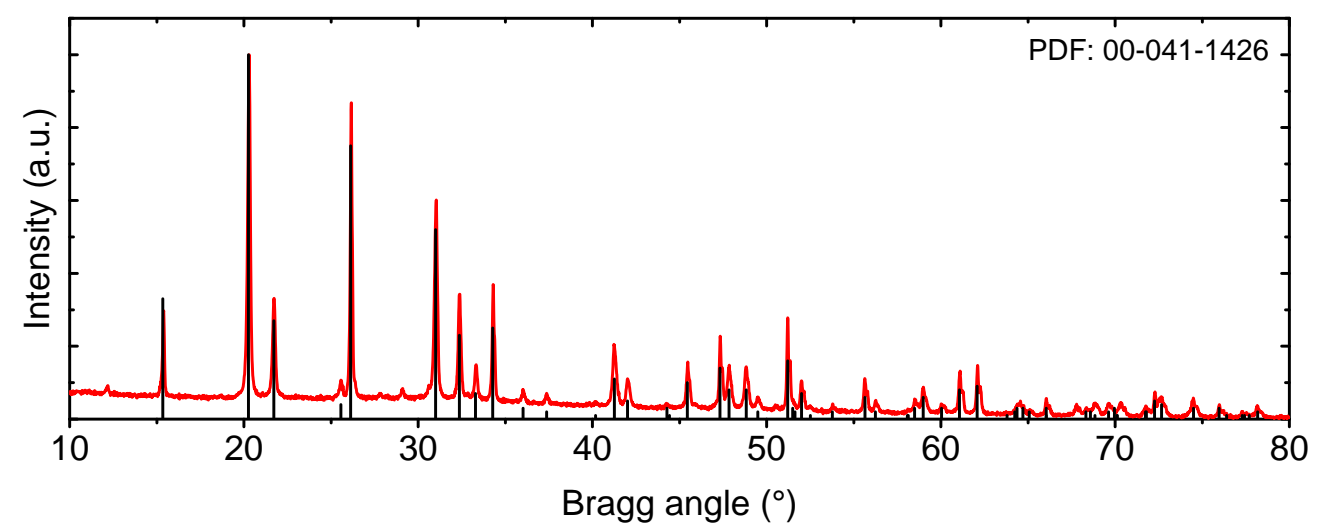

FIGURE 4.5: X-ray diffractogram of the $\mathrm{V}_{2} \mathrm{O}_{5}$ nanofibres, overlaid with one of the ICDD patterns for orthorombic $\mathrm{V}_{2} \mathrm{O}_{5}$.

\subsection{Device fabrication and testing}

\subsubsection{Electrode and cell fabrication}

The construction of a prototype device requires the $\mathrm{V}_{2} \mathrm{O}_{5}$ NFs to be processed into an electrode. Despite the notable improvements in the overall structure of the nanofibres, the mechanical fragility of the material was still such that its use as a self-standing electrode was not feasible: after the calcination step, the fibres were very brittle and tend to crumble into a powder very easily. Therefore, the material needed to be manipulated and cast into a thin film.

The first step of electrode fabrication therefore consisted of processing the nanofibres into a slurry. Although polyvinylidene fluoride (PVDF) is usually the most commonly used binder polymer for electrodes, previous literature had expressed some concerns regarding its chemical reactivity with the electrolyte. ${ }^{18}$ For this reason, polytetrafluoroethylene (PTFE) was instead used as the binder. Because stainless steel was also reported to be incompatible with the electrolyte ${ }^{19}$ nickel foil was initially used as the current collector. However, it was observed that nickel is not a valid current collector metal, as it was also affected by corrosion from the electrolyte. Ultimately, Mo foil was chosen as the current collector, as it did not show any sign of reaction with the electrolyte.

Following electrode fabrication, the prototype devices were assembled for electrochemical testing. Swagelok-type cells in a two-electrode configuration (see 
also Section 3.4.2) were used, with the assembly taking place in an nitrogen-filled glovebox to avoid atmospheric contamination. Once assembled and taken outside the glovebox, the devices exhibited an open circuit voltage of $\sim 1.7-1.8 \mathrm{~V}$, which is consistent with those reported by previous literature. ${ }^{6}$ Furthermore, when shortcircuited, a current flow of a few hundred $\mu \mathrm{A}$ could be measured: this indicates that the battery is likely in a "charged" state, and a spontaneous electrochemical process takes place when the circuit is closed. These observation confirmed that the devices were indeed working as intended; therefore, detailed electrochemical testing was then performed.

\subsubsection{Electrochemical tests}

A simple galvanostatic charging-discharging sequence was used to test the devices. 20 discharge-charge cycles in the voltage range between $2.2 \mathrm{~V}$ and 0.02 $\mathrm{V}$ were performed on the cell, using a current density of $125 \mathrm{mAh} \mathrm{g}^{-1}$. The same cycling rate reported in the work by Jayaprakash et al. ${ }^{6}$ was used, as a way to compare the performance of the devices fabricated using $\mathrm{V}_{2} \mathrm{O}_{5}$ to the ones reported in the paper. Although the aforementioned publication used an upper voltage limit of $2.45 \mathrm{~V}$, it was found that more consistent results could be obtained by using lower voltage limits (see also Figure A.4). In addition, the same test was also performed on devices made using commercial, non-nanostructured $\mathrm{V}_{2} \mathrm{O}_{5}$. Typical results of such test are shown below (Figure 4.6).

A few important observation can be made from these graphs:

- The discharge profile shows a wide plateau at approximately $0.5 \mathrm{~V}$, while the charging curve displays two plateaus: one at about $1.1 \mathrm{~V}$ and one beyond $2 \mathrm{~V}$. This shape is somewhat similar to other charge-discharge profiles reported for $\mathrm{V}_{2} \mathrm{O}_{5}$ cathodes in the literature. ${ }^{6,18}$

- The specific capacity observed in these tests is significantly lower than what was reported by the authors of the paper by Jayaprakash et al. ${ }^{6}$

- The capacity decreases dramatically after each cycle, going from $63 \mathrm{mAh} \mathrm{g}^{-1}$ in the first cycle, to about $19 \mathrm{mAh} \mathrm{g}^{-1}$ in the $20^{\text {th }}$ cycle, roughly corresponding to a 70\% capacity loss. This decay is steeper in the first 8-10 cycles, and tends to stabilise in the remaining cycles. The decrease also correlates to a 

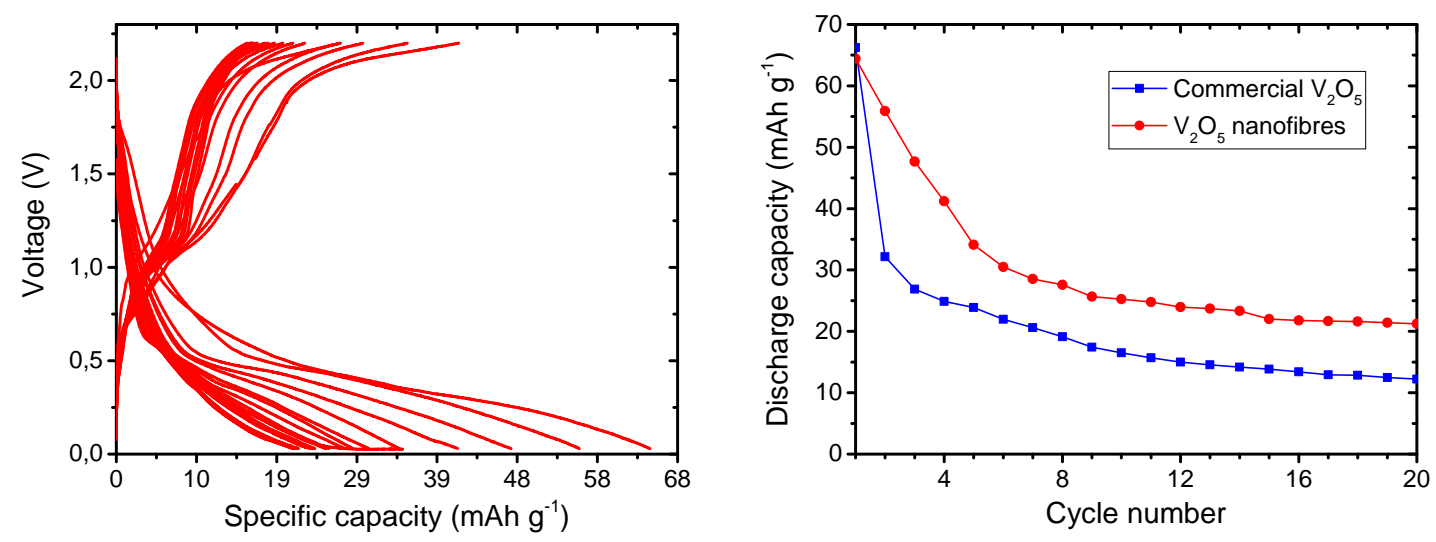

FIGURE 4.6: (Left) Galvanostatic cycling profiles of a Swagelok-type cell built using $\mathrm{V}_{2} \mathrm{O}_{5}$ NFs as cathode. The voltage reported in this plot is the cell potential. (Right) Comparison between the cycling performances of an AIB device built using commercial $\mathrm{V}_{2} \mathrm{O}_{5}$ and one built using $\mathrm{V}_{2} \mathrm{O}_{5}$ nanofibres. The upper voltage cutoff was set to $2.2 \mathrm{~V}$ for both materials.

change in the shape of the discharge profile, with the plateaus becoming less defined with every subsequent cycle.

- The specific capacity for the charge sequence is significantly lower than the one for the corresponding discharge cycle, indicating poor coulombic efficiency. This is also somewhat different from the aforementioned report by Jayaprakash et al. ${ }^{6}$

The following explanation is proposed to explain the discrepancies between the results obtained for $\mathrm{V}_{2} \mathrm{O}_{5}$ NFs and previous literature work. Regarding the lower specific capacity obtained in our experiment, a certain difference in performance can be expected, as these experiments were performed in the initial phase of the $\mathrm{PhD}$ project. Without any previous experience in device fabrication and testing, its expected that a more experienced scientist may have had a more inherently fine-tuned process and obtained better results. Regardless, much of the literature on $\mathrm{V}_{2} \mathrm{O}_{5}$ cathodes for AIBs, including very recent works, show a large variation in the reported specific capacities. Typical results include discharge capacities reported as low as $45 \mathrm{mAh} \mathrm{g}^{-1}$ for the first cycle, even for the very same type of nanostructure used in the original publication by Jayaprakash et al. ${ }^{6,18,20,21}$ Therefore, it is not completely unlikely that the first reports ${ }^{6}$ of a specific capacity of 300 $\mathrm{mAh} \mathrm{g}^{-1}$ might have been somewhat over-representative.

The rapid decay of the specific capacity and poor coulombic efficiency could both 
be justified by a poor reversibility of the intercalation mechanism. $\mathrm{Al}^{3+}$ ions are inserted into the cathode material during discharge, however the high charge density of the ion is likely to cause a strong interaction with the crystal lattice of $\mathrm{V}_{2} \mathrm{O}_{5}$ and could make the deintercalation process less favourable. This would therefore correspond to a reduced specific capacity in the charging phase and a loss of discharge capacity in the subsequent cycle. This hypothesis was later verified in a publication by Gu et al. $2^{21}$ the authors reported that the intercalation of $\mathrm{Al}^{3+}$ ions can also cause the disruption of the crystal structure of $\mathrm{V}_{2} \mathrm{O}_{5}$ and the subsequent trapping of the intercalated ions, leading to a loss in capacity and efficiency.

Although the result of the galvanostatic charge-discharge tests were not as positive as initially predicted, an important finding was still made: when the same cycling protocol was applied to devices built using commercially available, nonnanostructured $\mathrm{V}_{2} \mathrm{O}_{5}$, the discharging capacity would decrease even more rapidly, and stabilise to a lower bottom value than the one relative to the devices made with $\mathrm{V}_{2} \mathrm{O}_{5} \mathrm{NFs}$ (see also Figure 4.3.2 (right)). This can be interpreted as a preliminary indication of the advantage of using nanostructured cathodes in AIBs.

\subsection{Conclusions}

In summary, a novel protocol for the fabrication of $\mathrm{V}_{2} \mathrm{O}_{5}$ nanofibres was successfully developed, and the material was implemented as cathode in the fabrication of prototype AIBs. The results of the electrochemical tests performed on these devices, however, proved to be somewhat underwhelming, as the performance reported in the relevant literature could not be matched, let alone surpassed. Nevertheless, the experiments described in this chapter still serve as a proof-ofconcept of the benefits of using nanostructured cathode materials over bulk materials. Based on this result, the research project was continued by seeking other nanostructured materials to be used as cathodes for AIBs.

\subsection{Experimental section}

All the chemicals mentioned in this section were purchased from Sigma-Aldrich and used without any additional treatment, unless specified otherwise. Al foil 
was purchased from MTI Corp.

SEM micrographs were acquired with a JEOL-JSM6500F field emission scanning electron microscope, using an accelerating voltage of $20 \mathrm{kV}$. In order to enhance imaging contrast, samples were sputter-coated with a $10 \mathrm{~nm}$ Pt layer.

TEM micrographs were acquired with a JEOL-JEM2100F transmission electron microscope, operating at an accelerating voltage of $200 \mathrm{kV}$. Samples were prepared by dispersing the samples in ethanol via ultrasonication and drop casting onto a lacey carbon grid.

FT-IR spectra were acquired using a BRUKER ALPHA II FT-IR spectrometer using an attenuated total reflectance (ATR) module, in a wavenumber range between 4000 and $400 \mathrm{~cm}^{-1}$.

X-ray diffractograms were acquired using a PANalytical X'pert PRO XRD diffractometer operating in reflection mode, using a $\mathrm{Cu} \mathrm{K} \alpha$ X-ray source, in the Bragg angle range between 10 and 80 degrees. Samples were prepared by lightly grinding and dispersing the materials on a zero-background sample holder.

Galvanostatic charge-discharge experiments were performed using a NEWARE BTS CT-4008-5V10mA-164 (MTI Corp.) battery analyser system.

\section{Fabrication of $\mathrm{V}_{2} \mathrm{O}_{5} \mathrm{NFs}$}

A precursor solution was prepared by adding $0.8 \mathrm{~mL}$ of VOTIP and $0.4 \mathrm{~g}$ of PVP (average $\mathrm{M}_{w}: 1,300,000$ ) to $6 \mathrm{~mL}$ of isopropyl alcohol under magnetic stirring. The solution was then loaded into a plastic syringe with a stainless steel, 18-gauge, blunt tip needle. The electrospinning process was then performed using a voltage of $15 \mathrm{kV}$, a flow rate of $1 \mathrm{~mL} /$ hour, and a tip-to-collector distance of $13 \mathrm{~cm}$. A rotating drum collector was used in the process to optimise the form factor of the nanofibre mats. The resulting fibres were calcined in air using a muffle furnace; the temperature programme consisted in heating at a rate of $5{ }^{\circ} \mathrm{C} /$ minute until reaching $500{ }^{\circ} \mathrm{C}$, holding the temperature for 2 hours, and then cooling naturally to room temperature. After the heat treatment, the nanofibres were obtained in roughly $85 \%$ yield with respect to the vanadium content in the precursor solution. 


\section{Electrolyte preparation}

[EMIm]Cl and $\mathrm{AlCl}_{3}$ were baked at $100{ }^{\circ} \mathrm{C}$ in a vacuum oven for 48 hours to remove its water content, and immediately brought inside a nitrogen-filled glovebox with the $\mathrm{O}_{2}$ and $\mathrm{H}_{2} \mathrm{O}$ levels kept below $1 \mathrm{ppm}$. The electrolyte was prepared by gradually adding 1.3 equivalents of anhydrous $\mathrm{AlCl}_{3}$ to 1 equivalent [EMIm] Cl under magnetic stirring inside the aforementioned glovebox. An exothermic reaction takes place, after which a brown, slightly viscous liquid is formed.

\section{Device construction}

Electrode slurries were prepared by combining either the $\mathrm{V}_{2} \mathrm{O}_{5} \mathrm{NFs}$ or commercial $\mathrm{V}_{2} \mathrm{O}_{5}$ powder with PTFE binder and carbon black in a 8:1:1 mass ratio, and adding about $400 \mu \mathrm{L} / \mathrm{mg}$ of NMP. The mixture was first degassed using an ultrasonic bath, and then homogenised using a magnetic stirrer for at least 24 hours. In order to prepare the cathodes, the slurries were doctor bladed on Mo foil, which was then heated overnight at $120^{\circ} \mathrm{C}$ in a vacuum oven to evaporate the NMP dispersant. The electrodes were then cut into $11 \mathrm{~mm}$ diameter discs using a hole punch to be used in the devices.

Prototype batteries were assembled using a custom-built Swagelok-type cell consisting of a cylindrical PEEK casing with an inner diameter of $12 \mathrm{~mm}$ and two Mo rods as the current collectors (see also Section 3.4.2). All the components of the device were first baked at $100{ }^{\circ} \mathrm{C}$ in a vacuum oven for at least 2 hours to remove any residual adsorbed water, then immediately transferred inside a nitrogen-filled glovebox with the $\mathrm{O}_{2}$ and $\mathrm{H}_{2} \mathrm{O}$ levels kept below $1 \mathrm{ppm}$. One of the previously prepared $\mathrm{V}_{2} \mathrm{O}_{5}$-coated discs was used as cathode, and $11 \mathrm{~mm}$ diameter, high purity $\mathrm{Al}$ foil discs were used as the anode. Glass microfibre (GF/D) discs with 12 $\mathrm{mm}$ diameters, soaked in approximately $300 \mu \mathrm{L}$ of electrolyte, were used as separators. The devices were then wrapped with Parafilm as an additional moisture barrier and taken outside the glovebox for electrochemical testing. 


\section{Bibliography}

${ }^{1}$ G. C. Bond and S. F. Tahir, "Vanadium oxide monolayer catalysts Preparation, characterization and catalytic activity," Applied Catalysis, vol. 71, pp. 1-31, Apr. 1991.

${ }^{2}$ H. Jerominek, F. Picard, and D. Vincent, "Vanadium oxide films for optical switching and detection," Optical Engineering, vol. 32, pp. 2092-2100, Sept. 1993.

${ }^{3}$ X. Liu, J. Zeng, H. Yang, K. Zhou, and D. Pan, "V2O5-Based nanomaterials: synthesis and their applications," RSC Advances, vol. 8, pp. 4014-4031, Jan. 2018.

${ }^{4}$ S. Tepavcevic, H. Xiong, V. R. Stamenkovic, X. Zuo, M. Balasubramanian, V. B. Prakapenka, C. S. Johnson, and T. Rajh, "Nanostructured Bilayered Vanadium Oxide Electrodes for Rechargeable Sodium-Ion Batteries," ACS Nano, vol. 6, pp. 530-538, Jan. 2012.

${ }^{5}$ E. Uchaker, Y. Z. Zheng, S. Li, S. L. Candelaria, S. Hu, and G. Z. Cao, “Better than crystalline: amorphous vanadium oxide for sodium-ion batteries," Journal of Materials Chemistry A, vol. 2, no. 43, pp. 18208-18214, 2014.

${ }^{6}$ N. Jayaprakash, S. K. Das, and L. A. Archer, "The rechargeable aluminum-ion battery," Chemical Communications, vol. 47, no. 47, pp. 12610-12612, 2011.

${ }^{7}$ T. J. Macdonald, D. D. Tune, M. R. Dewi, C. T. Gibson, J. G. Shapter, and T. Nann, “A TiO2 Nanofiber-Carbon Nanotube-Composite Photoanode for Improved Efficiency in Dye-Sensitized Solar Cells," ChemSusChem, vol. 8, pp. 3396-3400, Oct. 2015.

${ }^{8}$ G. Wee, H. Z. Soh, Y. L. Cheah, S. G. Mhaisalkar, and M. Srinivasan, "Synthesis and electrochemical properties of electrospun V2O5 nanofibers as supercapacitor electrodes," Journal of Materials Chemistry, vol. 20, pp. 6720-6725, Aug. 2010.

${ }^{9}$ D. Yu, C. Chen, S. Xie, Y. Liu, K. Park, X. Zhou, Q. Zhang, J. Li, and G. Cao, “Mesoporous vanadium pentoxide nanofibers with significantly enhanced Li-ion storage properties by electrospinning," Energy \& Environmental Science, vol. 4, pp. 858-861, Mar. 2011.

${ }^{10}$ Y. L. Cheah, N. Gupta, S. S. Pramana, V. Aravindan, G. Wee, and M. Srinivasan, "Morphology, structure and electrochemical properties of single phase electrospun vanadium pentoxide nanofibers for lithium ion batteries," Journal of Power Sources, vol. 196, pp. 6465-6472, Aug. 2011.

${ }^{11}$ H.-G. Wang, D.-L. Ma, Y. Huang, and X.-B. Zhang, "Electrospun V2O5 Nanostructures with Controllable Morphology as High-Performance Cathode Materials for Lithium-Ion Batteries," Chemistry - A European Journal, vol. 18, pp. 8987-8993, July 2012.

${ }^{12}$ V. Modafferi, S. Trocino, A. Donato, G. Panzera, and G. Neri, "Electrospun V2O5 composite fibers: Synthesis, characterization and ammonia sensing properties," Thin Solid Films, vol. 548, pp. 689-694, Dec. 2013.

${ }^{13}$ B. Yan, X. Li, Z. Bai, M. Li, L. Dong, D. Xiong, and D. Li, “Superior lithium storage performance of hierarchical porous vanadium pentoxide nanofibers for lithium ion battery cathodes," Journal of Alloys and Compounds, vol. 634, pp. 50-57, June 2015.

${ }^{14}$ Z. Li, G. Liu, M. Guo, L.-X. Ding, S. Wang, and H. Wang, “Electrospun porous vanadium pentoxide nanotubes as a high-performance cathode material for lithium-ion batteries," Electrochimica Acta, vol. 173, pp. 131-138, Aug. 2015.

${ }^{15}$ Q. Liu, J. Zhu, L. Zhang, and Y. Qiu, "Recent advances in energy materials by electrospinning," Renewable E Sustainable Energy Reviews, vol. 81, pp. 1825-1858, Jan. 2018.

${ }^{16}$ J. Livage and C. Sanchez, "Sol-gel chemistry," Journal of Non-Crystalline Solids, vol. 145, pp. 11-19, Jan. 1992. 
${ }^{17}$ N. W. . E. K. S. . I. K. T. a. T. T. . R. K. T. a. J. H. K. H. National Institute of Advanced Industrial Science and Technology. ; NMR, M. Yanagisawa and O. Yamamoto ; MS, Spectral database for organic Compounds : SDBS. Tsukuba, Ibaraki, Japan : National Institute of Advanced Industrial Science and Technology, 1999-, 1999.

${ }^{18}$ H. Wang, Y. Bai, S. Chen, X. Luo, C. Wu, F. Wu, J. Lu, and K. Amine, “Binder-Free V2O5 Cathode for Greener Rechargeable Aluminum Battery," ACS Applied Materials \& Interfaces, vol. 7, pp. 8084, Jan. 2015.

${ }^{19}$ L. D. Reed and E. Menke, “The Roles of V2O5 and Stainless Steel in Rechargeable Al-Ion Batteries," Journal of the Electrochemical Society, vol. 160, no. 6, pp. A915-A917, 2013.

${ }^{20} \mathrm{H}$. Wang, S. Gu, Y. Bai, S. Chen, N. Zhu, C. Wu, and F. Wu, "Anion-effects on electrochemical properties of ionic liquid electrolytes for rechargeable aluminum batteries," Journal of Materials Chemistry A, vol. 3, pp. 22677-22686, Nov. 2015.

${ }^{21}$ S. Gu, H. Wang, C. Wu, Y. Bai, H. Li, and F. Wu, "Confirming reversible Al3+ storage mechanism through intercalation of $\mathrm{Al} 3+$ into V2O5 nanowires in a rechargeable aluminum battery," Energy Storage Materials, vol. 6, pp. 9-17, Jan. 2017. 
Chapter 5

Cathode materials: carbon nanofibres 



\subsection{Preface}

The second material investigated as part of this research project is electrospun carbon nanofibres (CNFs). The reason for picking this material comes, once again, from previous literature: a letter to the Nature journal. Published by Lin et al. in $2015,{ }^{1}$ the work presented an "ultrafast" aluminium-ion battery, using a "graphitic foam" cathode (Figure 5.1), which exhibited remarkable charging/discharge rates of up to $5000 \mathrm{~mA} \mathrm{~g}^{-1}$, while maintaining an operating voltage of roughly $2 \mathrm{~V}$, and a specific capacity of $\sim 60 \mathrm{mAh} \mathrm{g}^{-1}$. Such values correspond to a power density of up to $3000 \mathrm{~W} \mathrm{~kg}^{-1}$, a value on par with modern supercapacitors, and an energy density of $\sim 40 \mathrm{Wh} \mathrm{kg}^{-1}$, which is comparable to Ni-MH or lead-acid batteries. This impressive result indicates that nanostructured carbon materials can be a very appealing candidate for AIB cathodes; furthermore, not many literature reports of graphitic materials in AIBs were reported at the time this project was started. ${ }^{2}$ Therefore, the investigation of the use of nanocarbons in AIB cathodes was a very appealing research opportunity.

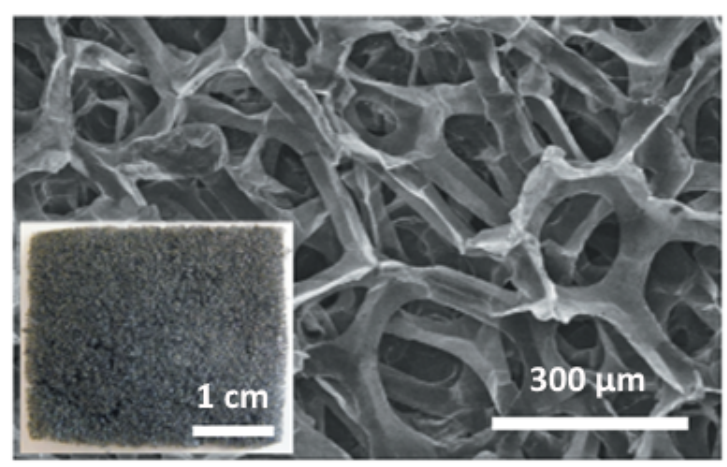

FIGURE 5.1: SEM micrograph of the graphitic foam used as cathode for AIBs by Lin et al. Inset: digital camera image of the material (adapted with permission from the publication) ${ }^{1}$

CNFs are extremely versatile materials, which can be synthesised easily by electrospinning and carbonising an appropriate precursor polymer. They are conductive, flexible, and can be readily functionalised. ${ }^{3}$ Therefore, it was hypothesised that using CNFs as a cathode for AIBs would present several advantageous features. First, thanks to their good mechanical properties, they could be used as "self-standing" electrodes without requiring the use of any additional substrates or current collectors. Furthermore, the degree of crystallinity of the nanofibres could be tuned from highly graphitic to completely amorphous, depending on 
the temperature used in the carbonisation process. Similarly, conductivity of the nanofibres can also be tuned. ${ }^{4}$ Lastly, they can be further chemically or physically modified to add additional functional groups or modify other properties, such as surface area or hydrophilicity. ${ }^{5}$ The following chapter describes the preparation of electrospun CNFs, their performance as electrodes for AIBs, and the efforts to improve them by increasing the degree of crystallinity of the material.

\subsection{Polyacrylonitrile-based nanofibres}

\subsubsection{Material preparation}

Typically, the most common way to prepare CNFs is to first electrospin a precursor polymer solution to obtain a free-standing mat of polymeric nanofibres. Polyacrylonitrile (PAN) is the most commonly used polymer for such purpose, as it has high carbon yield (greater than 50\%), and the ability to retain its nanomorphology during heat treatments. Next, in order to fixate the fibrous structure of the material, a stabilisation process consisting of heating the fibres at $200-300{ }^{\circ} \mathrm{C}$ in air is performed. This treatment causes the partial degradation of PAN to an intermediate with a ladder polymer-like molecular structure. ${ }^{6}$ (Figure 5.2)

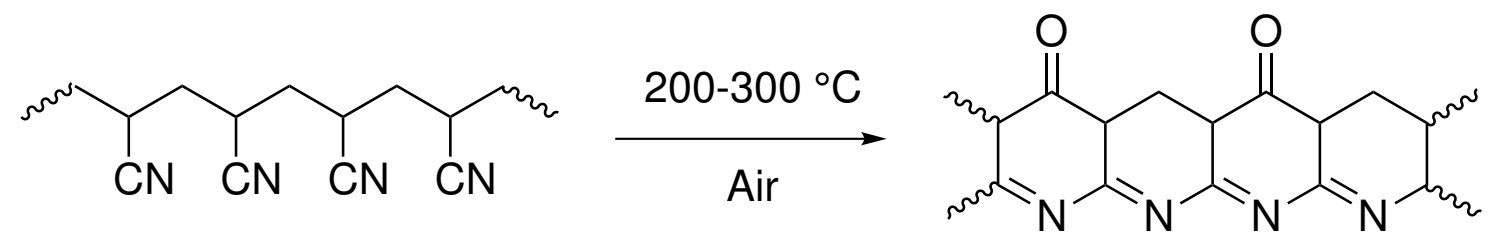

FIGURE 5.2: Diagram illustrating one of the possible mechanisms in which PAN can degrade into a ladder structure during the stabilisation treatment.

Finally, the carbonisation/graphitisation step is performed to convert the polymer to pure carbon through a pyrolysis reaction. The process is performed either under vacuum or an inert atmosphere, using temperatures of at least $600{ }^{\circ} \mathrm{C}$. The degree of graphitisation is correlated to the temperature of the process: a temperature of at least $1500-1600{ }^{\circ} \mathrm{C}$ is generally required for initial effects, and the best results are obtained at $>2500-2800{ }^{\circ} \mathrm{C} .{ }^{7,8}$ It is worth noting that some reports also use two separate steps for the high temperature treatment: First, the stabilised fibres are heated to an intermediate temperature between 1000 and 1500 
${ }^{\circ} \mathrm{C}$ (often called the "carbonisation" step), and then the final heating step at 2000$3000{ }^{\circ} \mathrm{C}$ (sometimes called the "graphitisation" step) is performed. ${ }^{9}$ Because of this, the "carbonisation/graphitisation" terminology used in the literature can be somewhat ambiguous, as it can either refer to a one-step or a two-step high temperature treatment process.

The preparation of precursor nanofibres was found to be relatively flawless: PAN was easily electrospun from a DMF solution into smooth nanofibres of about 300$400 \mathrm{~nm}$ diameters, as shown in Figure 5.3 (left). The free-standing mats were then stabilised by heating them in air at $220{ }^{\circ} \mathrm{C}$ for two hours. The material turned from colourless to dark brown (see also Figure A.6), suggesting its successful conversion to the aforementioned ladder structure. The increased conjugation in the polymer following the pyrolysis reaction likely leads to a narrowed band gap with increased UV-Vis light absorption. The mechanical properties remained mostly unchanged, as the mats retained their great flexibility. Finally, the nanofibres were carbonised at high temperature under an inert atmosphere. Based on the results reported in the literature, it was assumed that in order to work as a high performance cathode, the CNFs would need to have as high a degree of graphitisation as possible. As explained earlier in this section, the best results for graphitisation are achieved when a temperature of at least $1500-1600{ }^{\circ} \mathrm{C}$ is reached. Unfortunately, the equipment available did not allow any heat treatment at a temperature higher than $1400^{\circ} \mathrm{C}$. Nevertheless, two different calcination temperatures were tried, 900 and $1400{ }^{\circ} \mathrm{C}$, resulting in two samples: CNF-900 and CNF-1400.
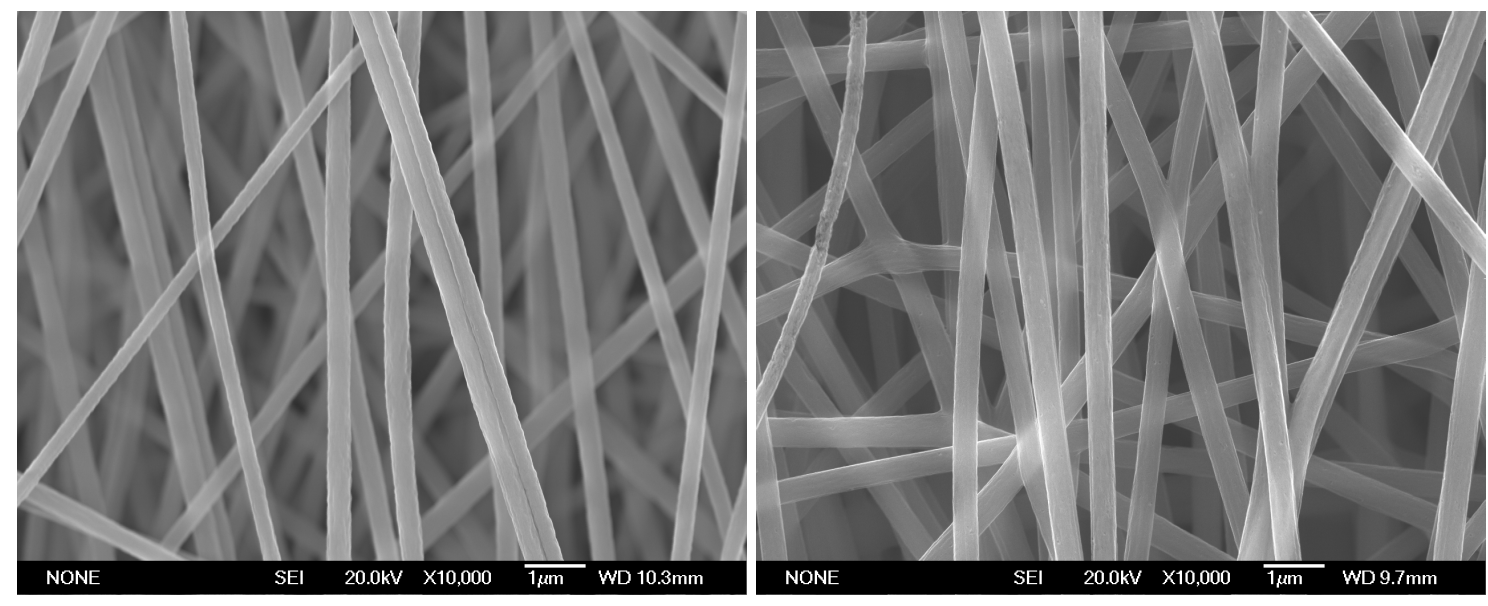

FIGURE 5.3: (Left) SEM micrograph of the precursor PAN nanofibres. (Right) SEM micrograph of the carbon nanofibres after the stabilisation and carbonisation treatment $\left(\right.$ at $\left.1400{ }^{\circ} \mathrm{C}\right)$. 
It is also worth noting that initial attempts at fabricating CNFs were also made using PVP as the precursor polymer. ${ }^{10}$ This strategy was however abandoned almost immediately, due to the extremely low carbon yield of PVP, and the disruption of its nanofibrous morphology after heat treatments (see also Figure A.5).

After the carbonisation process, the material appears as a black sheet; during this final step, the nanofibre mats tend to slightly shrink and stiffen, but still retaining a certain degree of flexibility (Figure A.6). On the nanoscale, the nanofibre diameters tend to decrease slightly, and their surface becomes less smooth and regular, with the presence of a few cavities and pinholes (see also Figure 5.3 (right)). The different carbonisation temperatures applied did not have a great impact on the nanomorphology of the nanofibres, with CNF-1400 appearing only slightly more porous and irregular than CNF-900 (see also Figure A.7).

EDX spectroscopy was also performed on CNF-1400 to gather information on the elemental composition of the material (Figure 5.4). The spectrum shows that carbon is largely the predominant element in the material, with a small peak relative to silicon, which is likely imputable to impurities or dust contamination in the SEM sample. A very weak oxygen $K_{a}$ peak is also seen, which is imputable to either defects in the carbon material (the stabilisation step in air could add oxygen moieties to the material, as seen in Figure 5.2), or dust contamination (in the form of silicon dioxide).

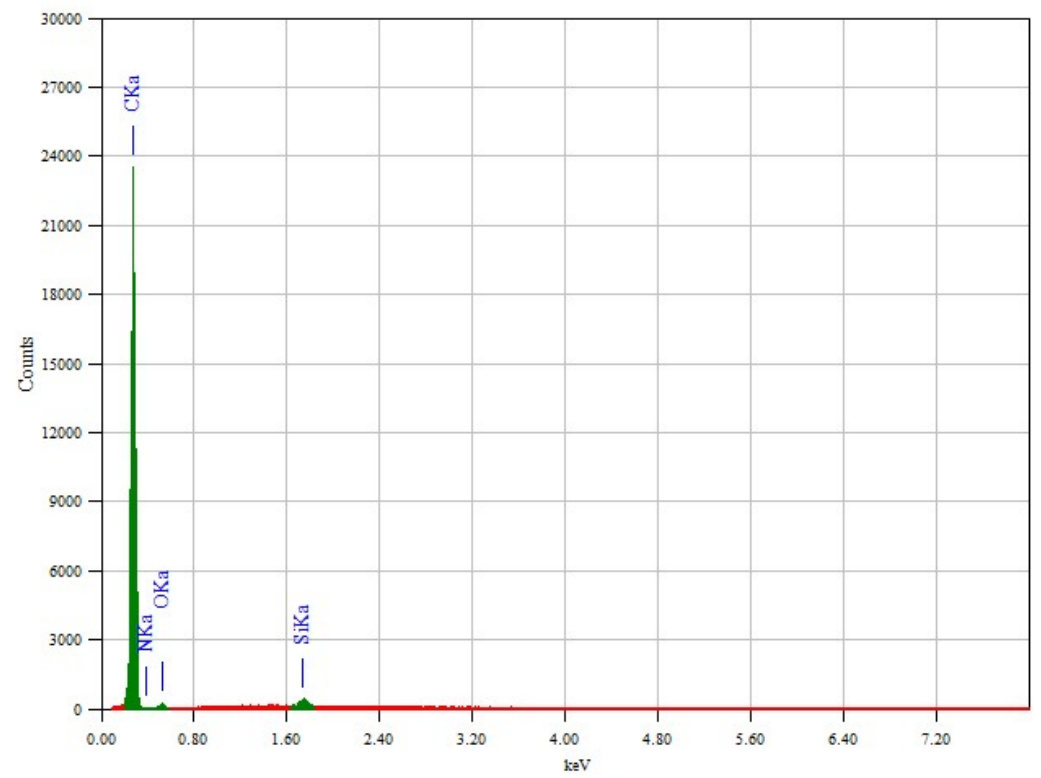

FIGURE 5.4: EDX spectrum of carbon nanofibres (carbonised at $1400{ }^{\circ} \mathrm{C}$ ). 


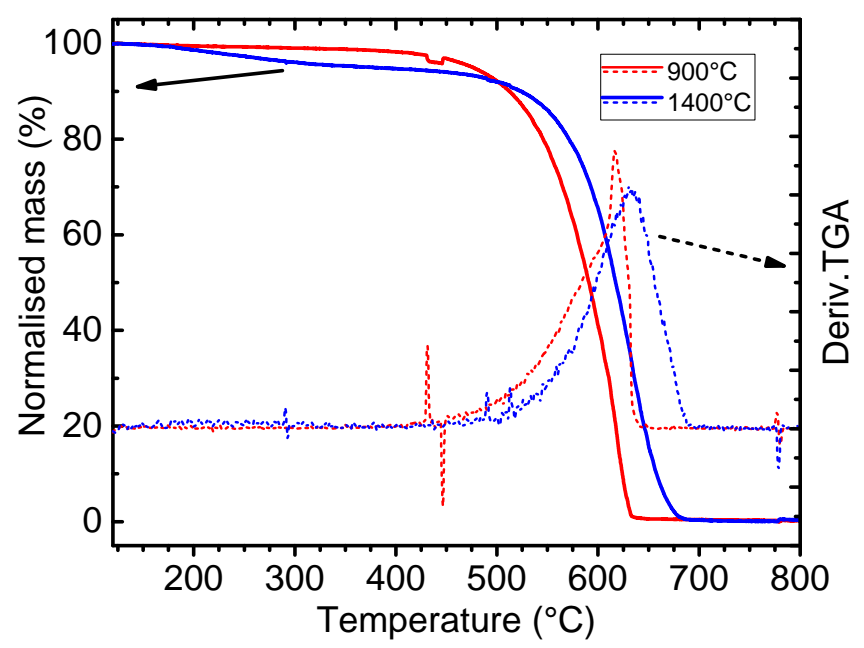

FIGURE 5.5: Thermogravimetric analysis (in air atmosphere) of the two carbon nanofibre samples.

Thermogravimetric analysis (TGA) in air was also performed on both CNF samples. Both appear fairly stable in air until about $600{ }^{\circ} \mathrm{C}$, with CNF-1400 decomposing at a temperature about $30^{\circ} \mathrm{C}$ higher than CNF-900. This result matches the prediction that a higher annealing temperature would lead to a more thermally stable material (Figure 5.5).

A Brunauer-Emmett-Teller (BET) surface area measurement was also performed on CNF-1400, yielding a relatively high surface area of about $47 \mathrm{~m}^{2} \mathrm{~g}^{-1}$.

While handling the samples, it was observed that CNF-900 is more flexible than CNF-1400. This was hypothesised to be due to a different degree of crystallinity in the fibres, and XRD measurements were therefore performed to verify the hypothesis. The diffractograms (Figure 5.6) predictably show only one very broad peak around $25^{\circ}$, which falls within the range of the (002) peak of graphite. The width of this peak, however, suggests that both samples are still mostly made of amorphous or semi-graphitic carbon, with the peak relative to CNF-1400 appearing only marginally narrower than CNF-900. This result was deemed not ideal for the intended purpose of our material, as it was hypothesised that a material with such low degree of crystallinity would likely not perform well as a cathode for AIBs. 


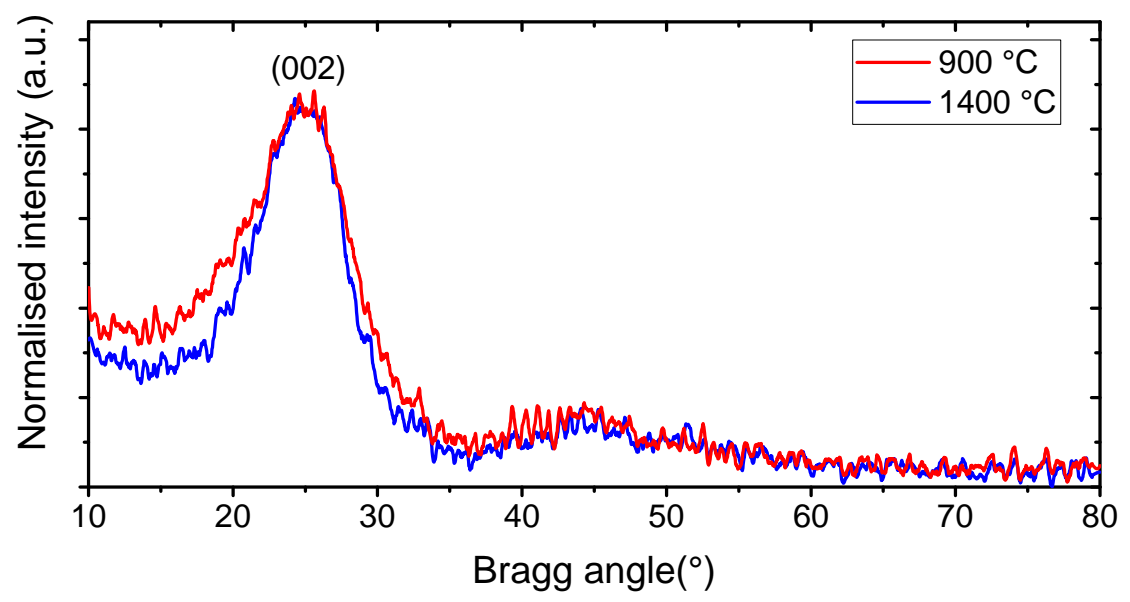

FIGURE 5.6: X-ray diffractograms of the two carbon nanofibre samples.

\subsubsection{Electrochemical tests}

Although the material presented non-ideal features for its intended purpose, an attempt to use CNFs as a cathodes for an AIB was made. This time, the assembly process proved to be relatively simple, as the mechanical properties of the material allowed it to be used as a self-standing electrode.As no additional processing was necessary, the CNF mats were simply cut into appropriately sized discs and used in the assembly of Swagelok-type cells. CNF-1400 was examined primarily due to its slightly higher graphitic character.

\section{Evidence for a solid-electrolyte interphase}

A galvanostatic test was initially performed on the cell. Assuming that the devices would work in a similar fashion as AIBs using graphitic cathodes (see also Section 2.4.2), the cycling programme was designed with a charging sequence first. For this specific test, a relatively medium rate of $100 \mathrm{~mA} \mathrm{~g}^{-1}$ was used, and a voltage range of 0.3 to $2.45 \mathrm{~V}$, which is the same potential range used in most publications on AIBs employing carbonaceous cathodes. This assumption, however, proved to be incorrect: it can be seen from Figure 5.7 (left) that the first charging step reaches a steady voltage plateau below the expected the cutoff voltage around $2.25 \mathrm{~V}$, which continues almost indefinitely. The exact voltage at which such plateau appears is somewhat variable between experiments and becomes progressively higher with the increase of the current rates, as shown by Figure 5.7 

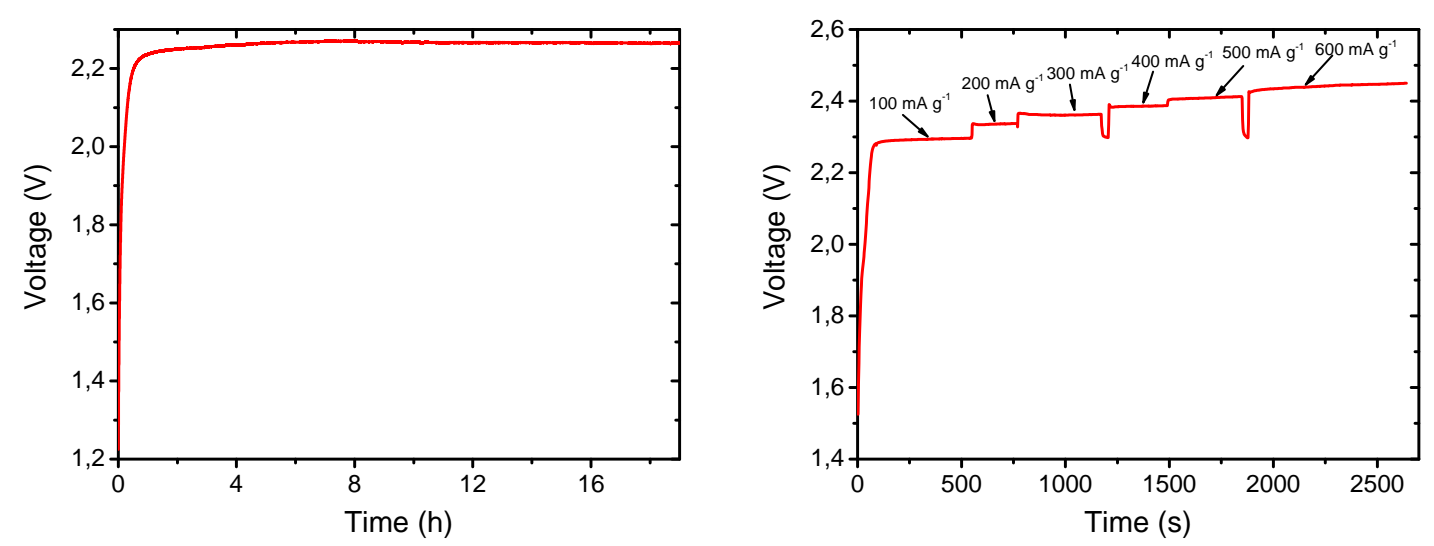

FIGURE 5.7: (Left) Typical galvanostatic charge profile (at $100 \mathrm{~mA} \mathrm{~g}^{-1}$ ) of a battery built using CNFs (carbonised at $1400^{\circ} \mathrm{C}$ ) as cathode. (Right) Charging profile of a battery built using CNFs (carbonised at $1400^{\circ} \mathrm{C}$ ) as cathode, at increasingly

high current rates. The voltage reported in these plots is the cell potential.

(right). Only at the fast rate of $600 \mathrm{~mA} \mathrm{~g}^{-1}$ does the voltage start to gradually increase until it reaches the pre-established cutoff of 2.45 V.This phenomenon likely indicates the presence of unwanted processes taking place in the device. One possible hypothesis could be that the material somehow promotes the degradation of the electrolyte at a lower voltage compared to graphitic materials. This in turn could cause the generation of insoluble side products, leading to the formation of a solid-electrolyte interphase (SEI). This is an unexpected result, as there are no detailed reports of the phenomenon in the literature.

In order to further investigate the phenomenon, one cell was disassembled after a long galvanostatic charging step (100 $\mathrm{mA} \mathrm{g}^{-1}$ for about 10 hours). The cathode was washed with water and ethanol to remove any residual electrolyte, and then SEM imaging was used to inspect the morphology of the material (Figure 5.8). The images show that the fibres are coated by a lumpy film, which could potentially consist of the decomposition products of the electrolyte. SEM-EDX analysis indicates that the material contains a significant amount of aluminium and chlorine (Figure 5.9 (top)), which appears to be mostly located in the newly formed film, as demonstrated by the elemental maps in Figure 5.9 (bottom). This finding is in good agreement with the hypothesis of the formation of an SEI at the cathode. It is worth noting that besides the the aforementioned water and ethanol, such film could also not be removed from the cathode through washing with a variety of protic, polar, and non-polar solvents. It therefore unlikely that 

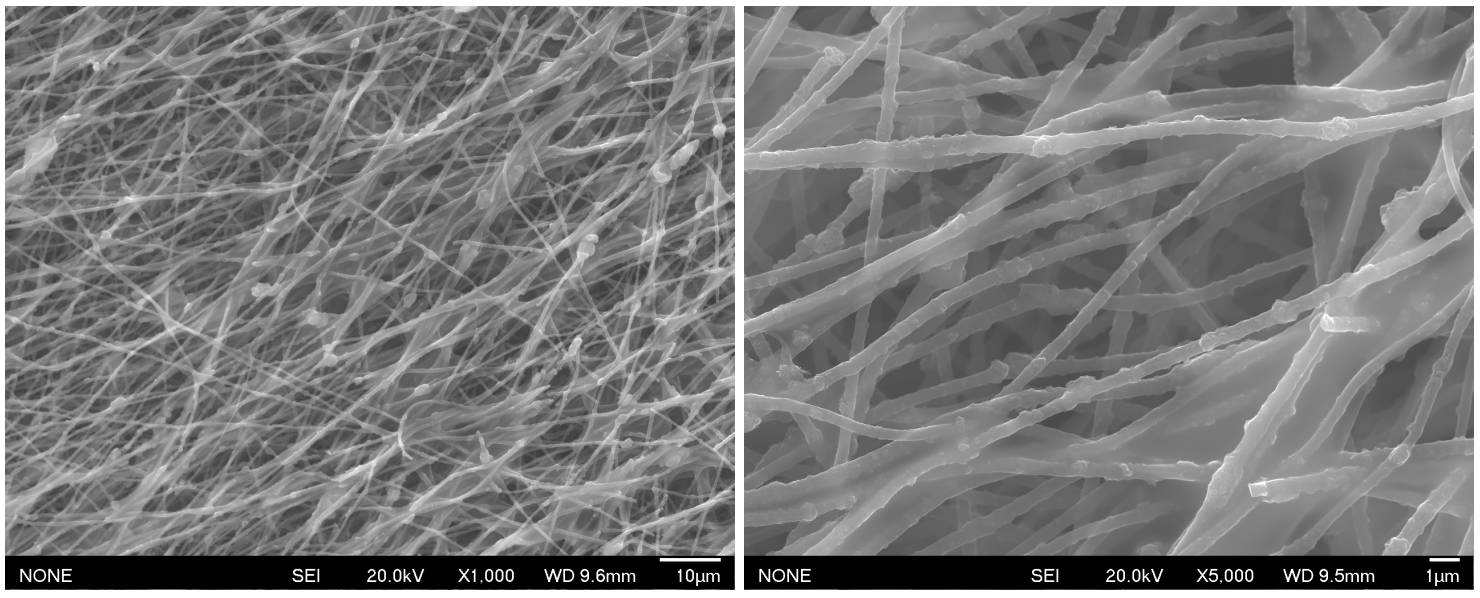

FIGURE 5.8: SEM micrographs of carbon nanofibres after charging at $100 \mathrm{~mA} \mathrm{~g}^{-1}$ for 10 hours.

this film is merely composed of residual [EMIm] $\mathrm{Cl} / \mathrm{AlCl}_{3}$ electrolyte, but instead it is probably caused by the decomposition of the species making up the ionic liquid. Furthermore, a control test was also performed by wetting a pristine CNF sheet in the electrolyte and rinsing with ethanol: SEM imaging revealed that no SEI-like film could be detected in the sample.

The formation of SEI has been confirmed to exist for graphitic materials when used as anodes for lithium-ion batteries. In these systems, the phenomenon takes place during the first charging of the device, when $\mathrm{Li}^{+}$ions are intercalated in the graphite anode. ${ }^{11}$ In the AIB system on the other hand, the intercalation mechanism follows an opposite pattern, as the negatively-charged $\mathrm{AlCl}_{4}^{-}$ions are inserted in the cathode during the charging phase (see also Equation 2.3a). Therefore, it is not unreasonable to speculate that the formation of an SEI on graphite would primarily happen during the first intercalation event, which corresponds to the first charging phase in both battery chemistries, regardless of the formal role of the carbonaceous electrode. For AIBs, this assumption is also supported by a possible voltage overlap between the intercalation reaction and the oxidation of the electrolyte. Figure 5.10 shows a typical cyclic voltammetry of an AIB using a pyrolytic graphite paper cathode.* A sharp increase in current can be observed above $2.2 \mathrm{~V}$. This indicates that the decomposition of the electrolyte could be in

*Pyrolytic graphite paper is a commonly used cathode material for AIBs. Its mechanical properties allow it to be directly cut and used as a self-standing electrode. Thanks to this, it has become a reliable "benchmark" electrode for AIB performance testing and comparison. 

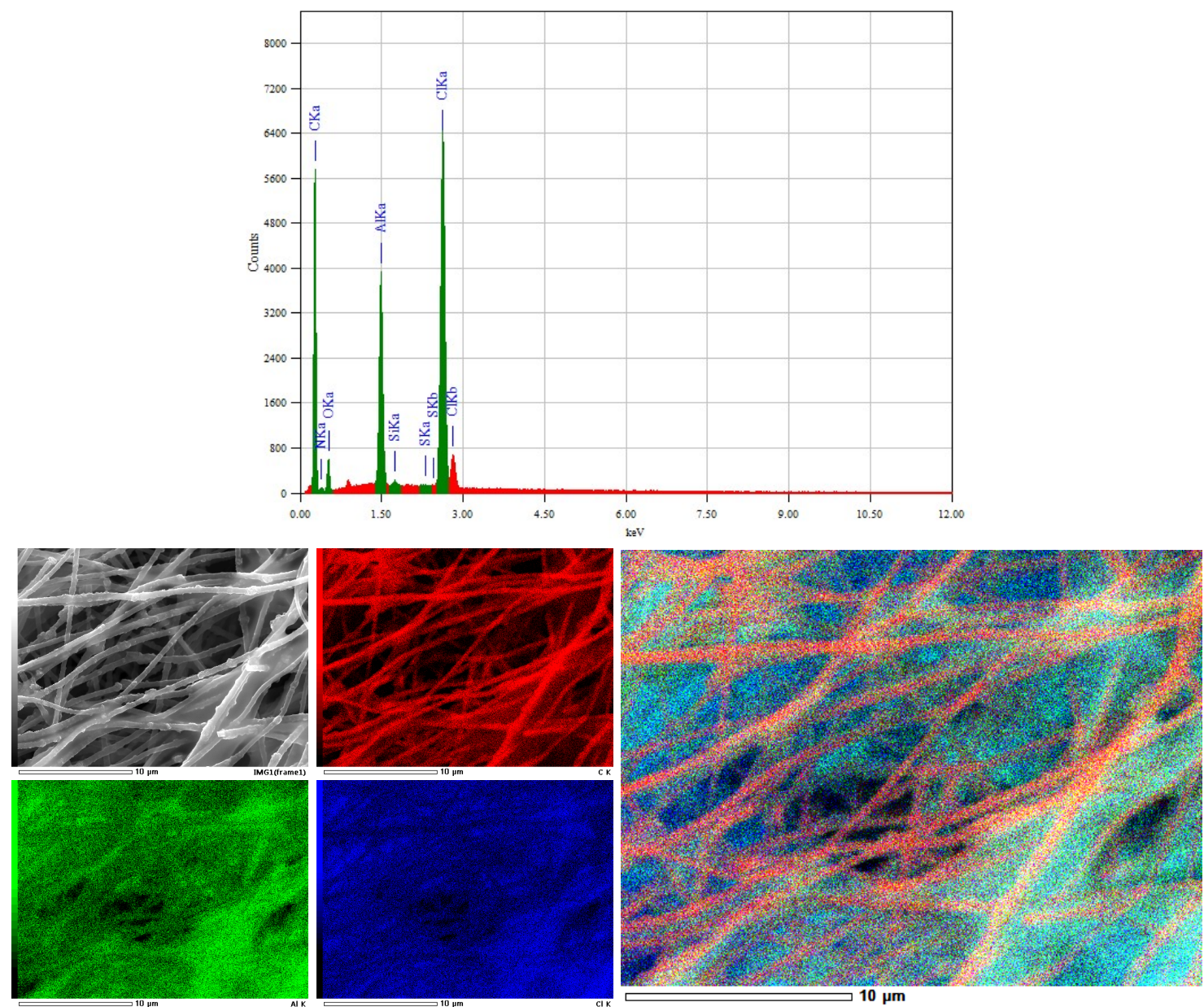

FIGURE 5.9: SEM-EDX data of carbon nanofibres after charging at $100 \mathrm{~mA} \mathrm{~g}^{-1}$ for 10 hours. (Top level) EDX spectrum of the sample. (Bottom level) SEM-EDX elemental maps: SEM image (top left), carbon (red), aluminium (green), chlorine (blue) maps, and overlay image (right).

part taking place at the cathode during the charging phase, even at potentials lower than the conventional cutoff value of $2.45 \mathrm{~V}$. In fact, although such voltage value has been adopted as the upper voltage limit in most publications using graphitic cathodes, it was initially determined empirically by Lin et al. ${ }^{1}$ for the "graphitic foam" cathode. The assumptions made for graphitic foam, however, might not be valid for any type of carbonaceous material, due to the difference in their physical and chemical properties, which could impact the potentials in which the intercalation and decomposition reactions take place. 


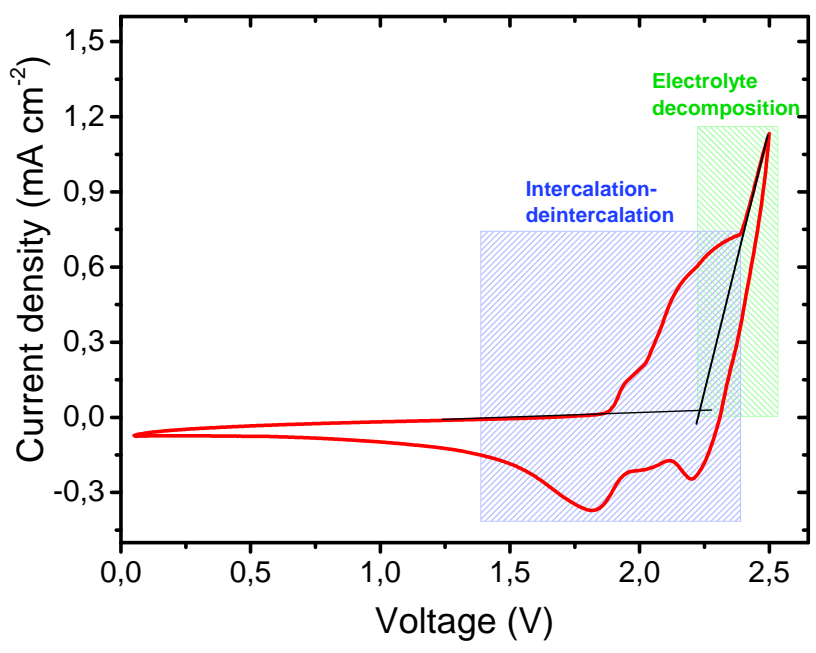

FIGURE 5.10: Cyclic voltammogram (third cycle, $10 \mathrm{mV} \mathrm{s}^{-1}$ ) of a Swagelok-type cell built using pyrolytic graphite paper as cathode, showing the possible voltage overlap between the intercalation reactions and the decomposition of the electrolyte. The current density is referred to the projected cathode area; the voltage reported in this plot is the cell potential.

Another observation from previous literature could also be linked to this potential overlap between the two reactions: when pyrolytic graphite paper is used as a cathode in AIBs, a considerably low coulombic efficiency is obtained in the first charge/discharge cycle. ${ }^{12,13}$ Such behaviour was also observed in tests performed in this thesis (Figure A.8). The previous publications reporting this phenomenon have attributed it to a decrease in the porosity of the electrode, caused by the volumetric expansion of the graphitic lattice in the first cycle. Although such explanation is well argued, the results of the aforementioned experiments suggest that the decomposition of the electrolyte and formation of SEI might also be contributing to the phenomenon. The same lumpy film observed in CNFs indeed also appears to be present in pyrolytic graphite paper cathodes after galvanostatic cycling, as demonstrated by the SEM figure and EDX spectrum in Figure A.9. One possible explanation for the phenomenon could be that a small quantity of surface defects are present in pyrolytic graphite paper, which could act as reaction centres and cause the premature oxidation of the electrolyte. Such unwanted process would therefore be responsible for the loss in efficiency during the first cycle, as part of the specific capacity obtained in the first charging step could be 
caused by the irreversible decomposition of the electrolyte species. In the subsequent cycles, the efficiency is recovered thanks to the newly-formed SEI layer, coating the graphite particles and shielding the surface defects. This hypothesis is also consistent with the fact that some graphitic materials in the literature show low coulombic efficiencies in the first cycle, ${ }^{13,14}$ while others do not display such behaviour. ${ }^{15,16}$ Because natural graphites are usually purchased in the form of a powder, they need to processed into a film and deposited onto a current collector by using something like PVDF as binder polymer. The carbon particles are therefore covered by a thin polymer layer, which could potentially shield the surface defects from entering in direct contact with the electrolyte, preventing its degradation and the formation of SEI. Pyrolytic graphite paper, on the other hand, is mostly used as a free-standing electrode; its surface is therefore exposed to the electrolyte more prominently, causing the degradation reaction.

This hypothesis is also further supported by a few key details in recent related publications. First, a paper by Stadie et al. describes the use of zeolite-templated carbon, a material with a reported extremely high surface area of $3510 \mathrm{~m}^{2} \mathrm{~g}^{-1}$, as a cathode for AIBs. ${ }^{17}$ Although the authors claim that no SEI formation was observed, all the galvanostatic cycling and cyclic voltammetry data reported in their work uses a maximum voltage of $2.2 \mathrm{~V}$, as opposed to the more conventionally used value of 2.4-2.45 V (see also Figure 2.3). Previous experiments (Figure 5.7) have shown that the process of SEI formation does indeed take place at values above $2.2 \mathrm{~V}$; therefore, it would not be unreasonable to speculate that zeolitetemplated carbon could show a similar type of behaviour at higher charging voltages. Furthermore, the cathode was shown to work primarily on a capacitive, surface adsorption-based mechanism, with negligible intercalation of $\mathrm{AlCl}_{4}^{-}$ions. XRD data from the publication revealed that the material possessed a low degree of graphitic character, which is similar to what was observed for CNFs. It is therefore possible that such low crystallinity is also the main cause of the reduction of the voltage window. Such hypothesis is also supported by the fact that amorphous or semi-graphitic carbons generally have a high amount of surface defects such as oxygen-containing functional groups (carbonyl, hydroxyl, carboxyl), non $s p^{2}$-hybridised carbon sites, and graphitic edges: such defects could possibly act as "catalytic sites", promoting the electrolyte degradation reaction. ${ }^{18,19}$ In 
addition, the high surface area of the material could further exacerbate this process by drastically increasing the density of reaction centres in the material. This is similar to what happens in the LIB system, where high surface areas in carbonaceous electrodes are correlated to thicker SEI films. ${ }^{20}$ More interesting information can be found in the original publication on the "ultrafast" AIB by Lin et al. ${ }^{1}$ Although this work uses high surface area graphitic foam as a cathode, a well-defined intercalation-based process with high coulombic efficiency could be observed in the material, excluding the likelihood of the formation of SEI. This behaviour can be explained by observing two factors: first, according to the literature method used to fabricate the graphitic foam cathode ${ }^{21}$ the material should possess a surface area roughly between 300 and $850 \mathrm{~m}^{2} \mathrm{~g}^{-1}$. Although this value is still considerably high, it is significantly lower to what reported by the aforementioned work by Stadie et al. Furthermore, XRD and Raman spectroscopy data from the paper show that the material is highly graphitic, with virtually no amorphous carbon or defects present. It is worth noting, however, that the graphitic foam cathode showed slightly worse coulombic efficiencies at lower current rates: therefore, it is not excluded that some similar undesired processes might be happening at higher charging voltages for such material, albeit in a less dramatic way. On the other hand, although CNFs appear to have much lower surface areas than zeolite templated carbons and graphitic foams (albeit still higher than bulk materials), they are composed entirely of amorphous or semi-graphitic carbon, as suggested by XRD (Figure 5.6) and Raman spectroscopy data (Figure A.10). These observations therefore suggest that the presence of amorphous carbon in the material could potentially be responsible for the early onset of electrolyte degradation and the formation of SEI. CNFs likely have the same surface defects mentioned in the case of zeolite templated carbon, ${ }^{18,19}$ which could act as active sites for the electrolyte degradation reaction. In addition, the moderately high surface area of the material could amplify the process by increasing the density of reaction centres in the material. It is unclear at this stage, however, what is the exact mechanism promoting the decomposition of the electrolyte; and further studies would be needed to bring more clarity on the subject.

X-ray photoelectron spectroscopy (XPS) was used to study the surface composition of CNFs both before and after the long charging step leading to SEI formation. It can be seen from Figure 5.11a that in the pristine CNF sample, the $\mathrm{C} 1 \mathrm{~s}$ narrow 


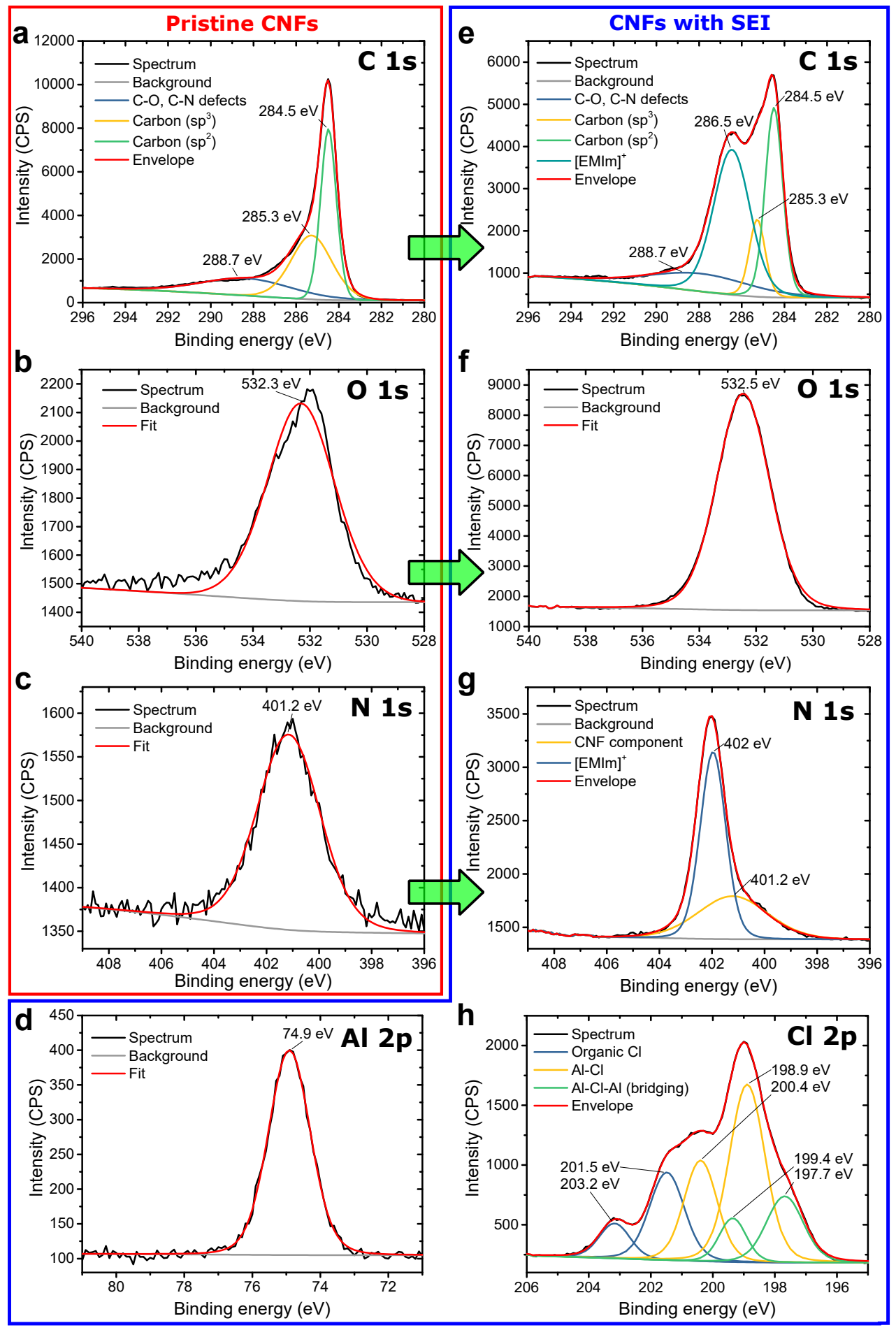

FIGURE 5.11: Deconvoluted XPS narrow scans of selected spectral regions for a CNF sample before (a-c) and after (d-h) charging at $100 \mathrm{~mA} \mathrm{~g}^{-1}$ for 10 hours: $\mathrm{C}$ 1s (a,e), O 1s (b,f), N 1s (c,g), Al 2p (d) and Cl 2p (h). 
scan shows a peak with highly asymmetrical line shape, indicating the presence of multiple chemical environments. The photoelectron spectrum was deconvoluted using a multiple peak fit, revealing the possible presence of three main components. The most intense peak, with a maximum at $284.5 \mathrm{eV}$, can be associated with bulk $s p^{2}$ carbon, likely the main component of the nanofibres. The second peak, with a maximum at $285.3 \mathrm{eV}$, is imputable to the presence of irregular or defective $s p^{3}$ carbon, consistent with our observations from XRD and Raman experiments. The positions of these two peaks are also in good agreement with other reports of similar carbon materials reported in the literature. ${ }^{22,23}$ Finally, a less intense and notably broader peak, centred at $288.7 \mathrm{eV}$, is also found. This peak is likely ascribable to the presence of carbon atoms bound to different, more electronegative elements such as oxygen and nitrogen. Previous literature indicates that this component is associated with the presence of functional groups such as hydroxyl, ether, nitrile, carbonyl, carboxyl or ester. ${ }^{24,25}$ The presence of heteroatomic defects is also supported by the narrow scans for the $\mathrm{O} 1 \mathrm{~s}$ and $\mathrm{N}$ 1s regions in Figure $5.11 \mathrm{~b}$ and Figure 5.11c, respectively. A fairly symmetrical peak in the binding energy range corresponding to the reported values for the aforementioned functional groups can be found in each of the two regions. ${ }^{24-26}$ XPS data therefore provides additional evidence of the presence of heteroatomic defects in the material, which could potentially be responsible for unwanted side reactions with the chloroaluminate species in the electrolyte, causing the formation of the SEI film. XPS was also performed to the charged CNF sample, to further study the composition of the SEI, and tentatively identify any new compounds created by electrolyte decomposition. The $\mathrm{C} 1 \mathrm{~s}$ narrow scan in Figure 5.11e shows that, in addition the same type of carbon environments observed for pristine CNFs (as indicated by the presence of peaks matching the positions of the peaks found in the pristine CNF sample, with comparable linewidth), a new prominent peak can be found in the deconvoluted spectrum with maximum at $286.5 \mathrm{eV}$. This matches well with the binding energies reported for the carbon atoms in alkyl-imidazolium cations, ${ }^{27,} 28$ suggesting that either the $[\mathrm{EMIm}]^{+}$ion or some of its derivatives are present in the film. This hypothesis is further supported by the N 1s narrow scan in Figure $5.11 \mathrm{~g}$, as a new intense peak centred at $402 \mathrm{eV}$ is found together with the previously existing one. Such a value is also in good agreement with literature reports of $\mathrm{N} 1 \mathrm{~s}$ 
photoelectron spectra for alkyl-imidazolium compounds. ${ }^{27,29}$ Interestingly, similar photoelectron spectra were acquired for charged graphite samples by a recent publication, ${ }^{12}$ but a different interpretation was proposed by the authors: the $\mathrm{C} 1 \mathrm{~s}$ peak at $286.5 \mathrm{eV}$ was attributed to either oxidised graphite (due to anion intercalation), or partial co-intercalation of [EMIm] ${ }^{+}$cation in the crystal lattice. However, given that the chemical information provided by XPS is highly surface-weighted, the hypothesis that such peaks are relative to species present on the surface of the sample (i.e. the SEI film) is more likely. Looking at the $\mathrm{O} 1$ s spectrum (Figure 5.11f), no particular new features are found. The intensity of the peak, however, is notably increased, suggesting the presence of new compounds, likely emerging from reactions involving atmospheric oxygen. Due to the large variety of plausible chemical species responsible for such peak, a clear interpretation of this phenomenon is not easy. A possible explanation is the presence of decomposition products deriving from the chloroaluminate ions, such as aluminium oxides or hydroxides in the samples. ${ }^{30,31}$ This hypothesis is also somewhat supported by the presence of a peak in the charged $\mathrm{Al} 2 \mathrm{p}$ spectrum. Centred at $74.9 \mathrm{eV}$ (Figure $5.11 \mathrm{~g}$ ), some possible compounds matching this range of binding energies include $\mathrm{AlCl}_{3}$, aluminium oxides, and aluminium hydroxides, which are consistent with the aforementioned hypothesis. ${ }^{30,31}$ Another very important piece of information can be deduced from the $\mathrm{Cl} 2 \mathrm{p}$ spectrum in Figure 5.11h: the highly irregular shape of the peak suggests the presence of multiple chemical states for chlorine. A curve fitting study reveals that the peak can be deconvoluted into three different chemical environments, as shown by the presence of three pairs of spin-orbit split peaks. These feature area ratios of approximately 1:2 and an energy splitting of about $1.6 \mathrm{eV}$, which is the expected lineshape of a single $\mathrm{Cl} 2 \mathrm{p}$ XPS environment. ${ }^{24}$ While the two environments situated at lower energies could be attributed to the $\mathrm{Cl}$ atoms in either terminal or bridging positions of the chloroaluminate ions, ${ }^{24,30}$ the higher energy doublet falls in the typical range of organic (alkyl- or acyl-) chlorides. This indicates that one of the possible electrolyte degradation mechanisms could involve the formation of carbon-chloride bonds between the chloroaluminate ions and either CNFs or the $[\mathrm{EMIm}]^{+}$cation.

Based on this data, it is therefore likely that both chloroaluminate species and their decomposition products are also present in the SEI film. A previous publication reports that that, when high potentials $\left(>2.5 \mathrm{~V} \mathrm{vs} \mathrm{Al}^{3+} / \mathrm{Al}\right)$ are applied to 
chloroaluminate electrolytes, the main mechanism of degradation is the oxidation of $\mathrm{AlCl}_{4}^{-}$ions generating chlorine gas, according to Equation 5.1:32

$$
4 \mathrm{AlCl}_{4}^{-} \longrightarrow 2 \mathrm{Al}_{2} \mathrm{Cl}_{7}^{-}+2 \mathrm{e}^{-}+\mathrm{Cl}_{2}(\mathrm{~g}) \uparrow
$$

If this reaction were to take place in our system, no new compounds would be generated at high potentials, so would not contribute to the formation of a solid film on the electrode. It is worth noting, however, that such equation refers to the oxidation of those species using a metallic tungsten electrode, with no organics or reactive functional groups present. Conversely, numerous such functional groups are potentially present in CNFs. Furthermore, because the chloroaluminate species present in the electrolyte (especially $\mathrm{Al}_{2} \mathrm{Cl}_{7}^{-}$) are known to exhibit Lewis acid behaviour, ${ }^{33,34}$ it is not unreasonable to speculate that they could form adducts with the functional groups of CNFs, which can serve as Lewis bases. Such adducts could further react by means of the oxidising potential applied to the electrode, giving rise to new, insoluble compounds and contributing to the formation of the SEI film. Therefore, the oxide and hydroxide compounds detected in the XPS spectrum could either be directly formed by reactions between the chloroaluminate ions and the surface functionalities of CNFs during the galvanostatic charging process, or as a further reaction of intermediate species present in the SEI film with ambient atmosphere during temporary handling of the samples outside the glovebox. Similarly, the presence of XPS peaks ascribable to imidazolium ions or its derivatives could be justified by two possible hypotheses: 1) residual [EMIm] ${ }^{+}$cations trapped in the SEI film, or 2) decomposition of such ions during the charging process; such reactions could be caused by one or more of the aforementioned factors, i.e. the presence of Lewis acids/bases and relative adducts, oxidising potentials applied to the cathode, and later exposure of the SEI film to atmospheric oxygen and water.

In order to better elucidate the electrochemical behaviour of a CNF material as a cathode in AIBs, a cyclic voltammetry experiment was performed in the voltage range of 0.05 to $2.5 \mathrm{~V}$, using a voltage ramp of $10 \mathrm{mV} \mathrm{s}^{-1}$ (Figure 5.12).

The voltammogram shows relatively weak oxidation and reduction peaks, emerging on top of a hysteretic baseline. A few key observation can be made from the voltammogram: 


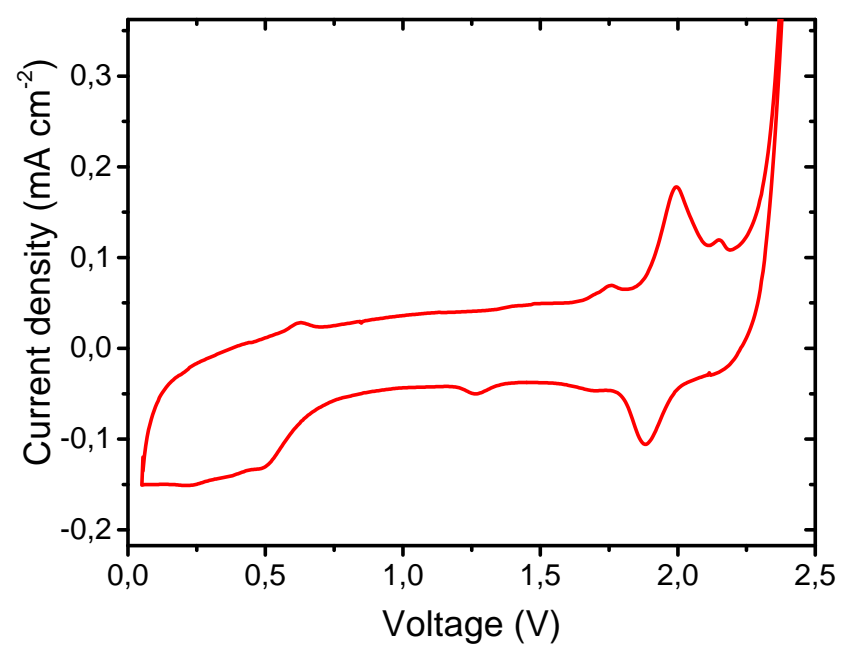

FIGURE 5.12: Cyclic voltammogram (third cycle) of a Swagelok-type cell built using CNFs (carbonised at $1400{ }^{\circ} \mathrm{C}$ ) as cathode. The current density is referred to the projected cathode area; the voltage reported in this plot is the cell potential.

- A sudden increase in current above $2.2 \mathrm{~V}$ can be observed. This coincides with the steady voltage plateau observed in the initial charging experiment (Figure 5.7), and very likely corresponds to the bulk oxidation of the electrolyte leading to the SEI formation. This early onset of electrolyte oxidation is notably different to the one observed when using more graphitic, lower surface area carbons such as pyrolytic graphite paper (see also Figures 5.10 and A.11).

It is also worth noting that such increase in current becomes notably less sharp after a long galvanostatic charging step is performed on the device, as observed in Figure A.12. This is consistent with the hypothesis of the SEI film shielding the surface defects, and thus inhibiting the further decomposition of the electrolyte, resulting in an expansion of the useful voltage window.

- A series of oxidation and reduction peaks can be observed between 1.7 and $2.2 \mathrm{~V}$. These are somewhat similar to what is observed in graphitic materials (see also Figure A.11), and are usually associated with the intercalation and deintercalation of $\mathrm{AlCl}_{4}^{-}$ions. ${ }^{16,35-37}$ Although these peaks are not directly overlapping with the current increase associated with the electrolyte decomposition, they fall in a narrow voltage range, which is close to the upper limit 
of the electrolyte stability window.

After a long galvanostatic charging step is performed on the device (Figure A.12), the intensity of these peaks is notably decreased. This is a somewhat unexpected result, but a possible explanation could be that the intercalation reaction could be now hindered by the newly formed SEI, or by a structural rearrangement of the cathode following the electrochemical process.

- Another set of weak oxidation and reduction peaks can be observed between 0.2 and $0.7 \mathrm{~V}$. These do not correspond with any processes taking place in graphitic materials in AIBs, and therefore are probably specific to a process happening exclusively in this material. It is currently unknown what such process might consist of, but the low intensity of the peaks suggests that it is likely negligible.

- The hysteretic behaviour indicates that the device works primarily through a capacitive or pseudo-capacitive process: ${ }^{38,39}$ in other words, the primary mechanism of energy storage is likely not the intercalation of $\mathrm{AlCl}_{4}^{-}$ions into the material, but it probably involves the mere adsorption of the ions on the surface of the electrode. This result fits well with the characteristics of our material: the low graphitic character of CNFs would not easily allow the reversible intercalation of ions, but its high surface area could favour the adsorption of a large quantity of ions on the electrode surface, making the capacitive behaviour the main process in the device.

Finally, in order to verify that the the high surface area of CNFs is a factor promoting the early onset of electrolyte degradation and the formation of SEI, a control experiment was performed. A monolithic, low-surface area carbon "film" (Figure A.13) was fabricated by drop-casting a PAN solution onto a glass surface, evaporating the solvent, and performing the same heat treatments used to prepare CNFs. Through this fabrication process, the material should be composed of the same type of amorphous or semi-graphitic carbon as CNFs, i.e. the surface of the material should also present the same type of defects, but its monolithic structure would correlate to a much lower surface area compared to the nanofibrous morphology of CNFs. After fabricating the carbon film, a device was assembled using the material as cathode, and electrochemical testing was performed. Figure 5.13 (left) shows that in the galvanostatic charge-discharge test performed at $100 \mathrm{~mA}$ $\mathrm{g}^{-1}$, the same current rate as the initial test on CNFs (Figure 5.7), the charging 
phase reaches the expected upper voltage limit very quickly, without any distinguishable plateau. Even at a much lower charging rate of $10 \mathrm{~mA} \mathrm{~g}^{-1}$, the charging step reaches the target voltage after a reasonable charging capacity. Furthermore, it can be seen from the cyclic voltammogram in Figure 5.13 (right) that the increase in current after $2.3 \mathrm{~V}$ is much less pronounced than the one observed for CNFs (Figure 5.12). These results therefore suggest that the carbon film does not promote the early onset of electrolyte degradation and formation of SEI, and a high surface area is also necessary in combination with the surface defects for the process to take place more prominently.

\section{Galvanostatic cycling of CNFs}

In light of the findings described in the previous section, new galvanostatic charge-discharge tests were performed, using an adjusted upper voltage cutoff. Figure 5.14 (left) shows typical charge-discharge profiles at different current rates. The plot shows that the charge and discharge curves follow a relatively featureless trend, with only the cycle performed at the slowest rate of $14 \mathrm{~mA} \mathrm{~g}^{-1}$ showing very weakly-defined plateaus at about 1.7 and $1 \mathrm{~V}$ for the discharge curve, and around $2 \mathrm{~V}$ for the charge curve. The best discharge performance was also obtained by cycling the device at that rate, yielding a specific capacity of about
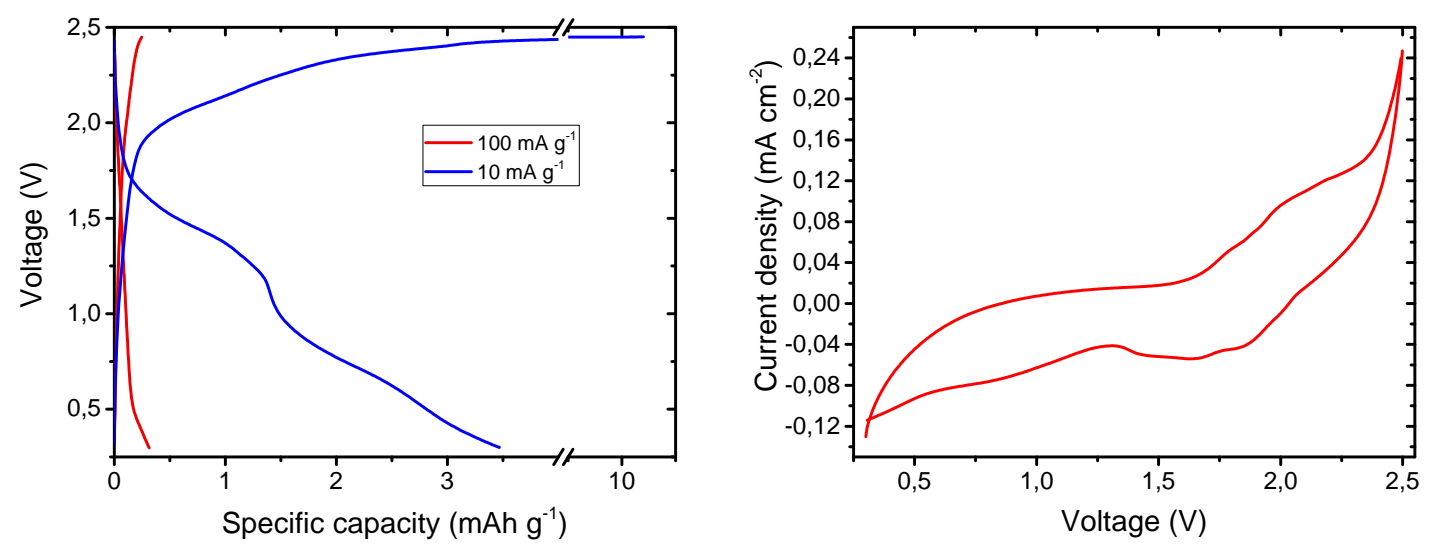

FIGURE 5.13: Electrochemical tests performed on a Swagelok-type cell built using a low-surface area carbon film (carbonised at $1400{ }^{\circ} \mathrm{C}$ ) as cathode: galvanostatic charge-discharge profiles (first cycle) (left) and cyclic voltammetry (third cycle) (right). The current density is referred to the projected cathode area; the voltage reported in these plots is the cell potential. 
$46 \mathrm{mAh} \mathrm{g}^{-1}$ relative to the first cycle. For comparison, the devices developed by Lin et al. ${ }^{1}$ achieved a stable discharge capacity of about $60 \mathrm{mAh} \mathrm{g}^{-1}$, and the best reports of devices using graphene or graphite-based cathodes claim a maximum specific capacity of 120-130 $\mathrm{mAh} \mathrm{g}^{-1} \cdot{ }^{35,40}$ This large gap in performance further confirms the material mostly operates via a capacitive behaviour. The two plateaus observed in the discharge curve are vaguely similar to those observed for graphitic materials, and therefore could potentially be linked to a small contribution from the intercalation and deintercalation of $\mathrm{AlCl}_{4}^{-}$ions into the $\mathrm{CNF}$ cathode. This assumption, however, is purely speculative, and further research is needed to confirm this hypothesis. At higher rates, the capacity decreases quite dramatically, and the plateaus disappear completely, suggesting a completely capacitive mechanism. The low overall specific capacity and poor capacity retention at higher rates of the material suggest that CNFs are not suitable for intercalation-based electrochemical energy storage, but works mainly on a capacitive or pseudo-capacitive mechanism.

It can also be seen from Figure 5.14 (right) that the coulombic efficiency is generally poor when the device is cycled at $14 \mathrm{~mA} \mathrm{~g}^{-1}$ but reaches $100 \%$ at higher current rates. This likely due to the electrolyte decomposition process partially taking place within the voltage range while cycling at a low rate, whereas a higher cycling current could shift the process to a higher potential past the voltage cutoff,
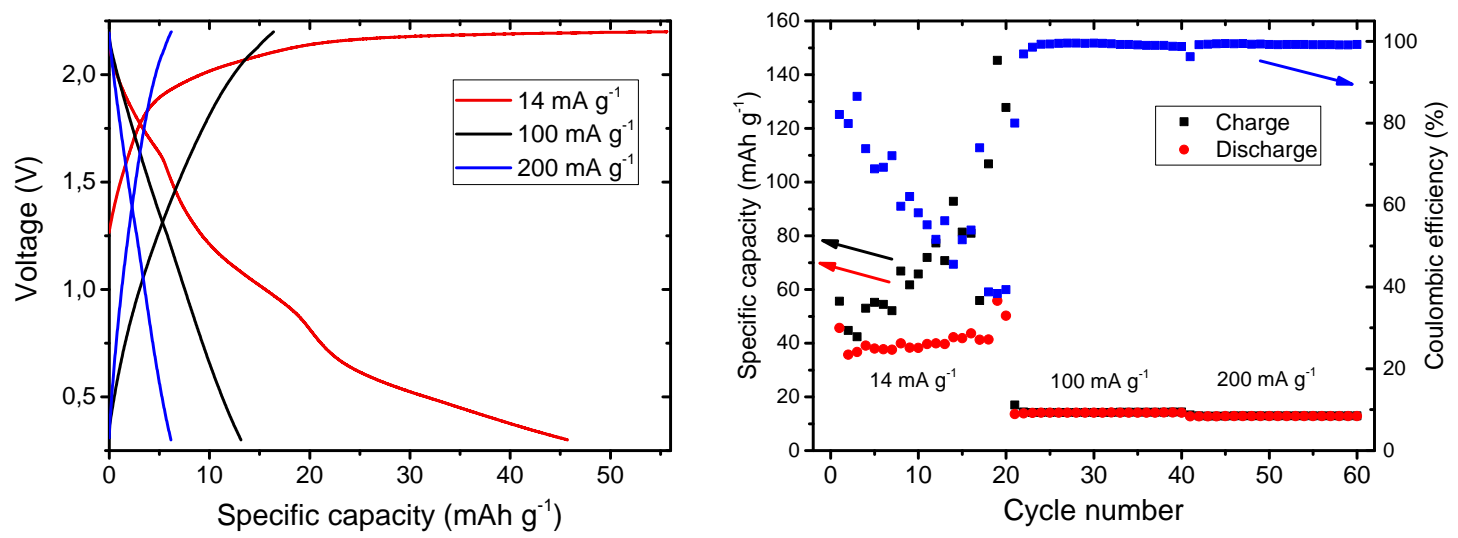

FIGURE 5.14: (Left) Typical galvanostatic charge-discharge profiles (First cycle) at various current rates of a battery built using CNFs (carbonised at $1400{ }^{\circ} \mathrm{C}$ ) as cathode. The voltage reported in this plot is the cell potential. (Right) Specific capacities and coulombic efficiencies of a typical galvanostatic charge-discharge test of a battery built using CNFs (carbonised at $1400^{\circ} \mathrm{C}$ ) as cathode. 
increasing the coulombic efficiency. Therefore, in order to maximise coulombic efficiency, the upper voltage cutoff would need to be optimised at each rate, in such way that the charging step is terminated before the voltage where the irreversible processes begin. However, this would likely have a negative repercussion on the specific discharge capacities, as the desired reversible processes ${ }^{\dagger}$ could also be partially suppressed. This is suggested by the difference in the shape of the discharge profiles between low and high current rates. The absence of plateaus in the $100 \mathrm{~mA} \mathrm{~g}^{-1}$ and $200 \mathrm{~mA} \mathrm{~g}^{-1}$ profiles in Figure 5.14 (left) suggest that at those rates, the process is likely purely capacitive. This is also supported by the proximity of the "intercalation" peaks in the 1.7-2.2 $\mathrm{V}$ range to that of electrolyte decomposition in the CV in Figure 5.12. An alternative solution to this problem would be to utilise a capacity-based cutoff instead of voltage-based; this way, a reasonable coulombic efficiency could be achieved at any given rate, without the need to sacrifice the increase in discharge capacity obtained by a slightly longer charging step. However, in order to optimise such protocol, the practical specific capacity of the cathode must be known with a high degree of accuracy.

This change in coulombic efficiency at different rates has been also reported by other publications, ${ }^{1}$ and can also be observed in the cycling data at "ultrafast" rates in Figure 5.15: in the first 100 cycles, performed at $1000 \mathrm{~mA} \mathrm{~g}^{-1}$, it can be seen that by reducing the upper cutoff from 2.45 to $2.44 \mathrm{~V}$, the coulombic efficiency increases by about $10 \%$. However, the specific discharge capacity also decreases by roughly $1 \mathrm{mAh} \mathrm{g}^{-1}$, corresponding to a roughly $8 \%$ loss. As the cycling rates get higher, the electrolyte decomposition reaction takes place at higher voltages. The voltage range can therefore be expanded, resulting in an increase of both discharge capacity and coulombic efficiency.

Overall, the results of the electrochemical tests described in this section suggest that CNFs mostly work through a capacitive or pseudo-capacitive mechanism, with little to no intercalation process taking place. This is likely due to the poor graphitic character of CNFs, which leads to two main issues:

1. The lack of a well-defined crystal lattice means that the material does not constitute a suitable "host" structure for the intercalation of $\mathrm{AlCl}_{4}^{-}$ions, which therefore are mostly interacting with its surface.

\footnotetext{
${ }^{\dagger}$ i.e. the hypothetically small quantity of intercalation reaction taking place in the cathode,
} contributing to the energy storage mechanism. 

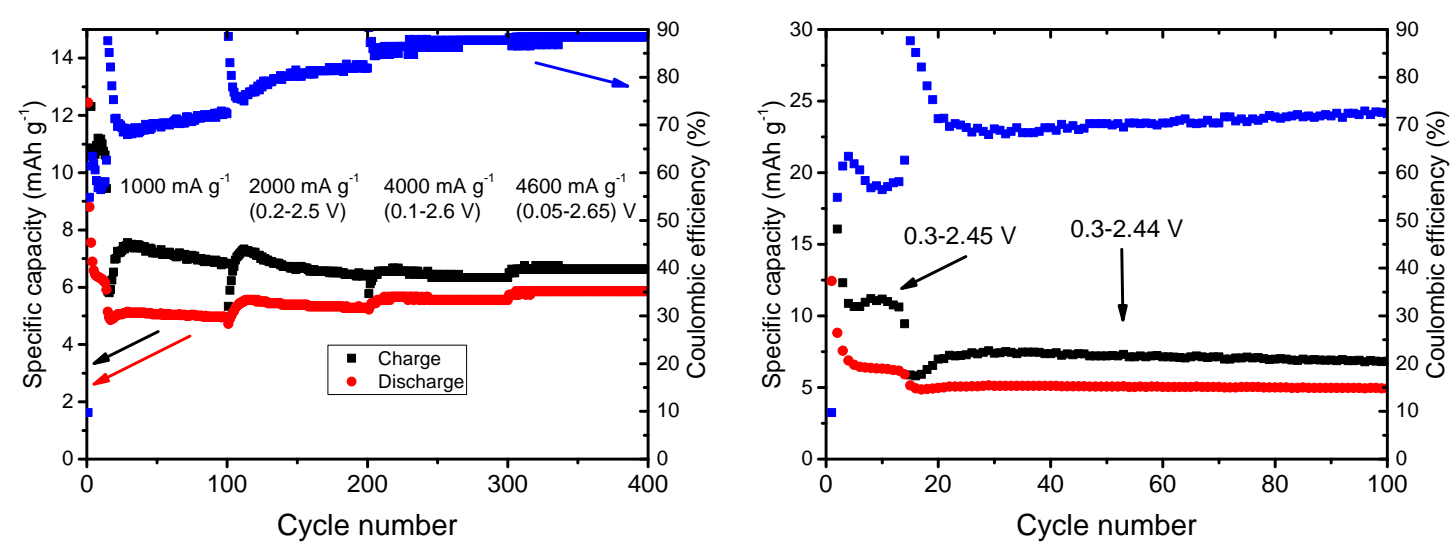

FIGURE 5.15: (Left) Specific capacities and coulombic efficiencies of a typical galvanostatic charge-discharge test at "ultrafast" current rates of a battery built using CNFs (carbonised at $1400^{\circ} \mathrm{C}$ ) as cathode. (Right) Detail of the first 100 cycles (using a rate of $\left.1000 \mathrm{~mA} \mathrm{~g}^{-1}\right)$.

2. The surface defects present in the material, related to the poor graphitic character, are likely causing the decomposition of the electrolyte at charging potentials higher than 2.2-2.3 V. Pushing the cell beyond such voltages promotes the formation of insoluble species, leading to the formation of an SEI. This causes poor coulombic efficiency in the devices, especially in the first cycle, and the inability to charge the battery devices at voltages higher than 2.2-2.3 V, which is where a significant portion of the desired reversible faradaic processes (i.e. intercalation) would likely take place.

\subsection{Enhanced carbon nanofibres}

Although a material showing capacitive behaviour is an interesting finding, it does not correspond to what was initially set as the main goal for this project. The evidence collected from previous experiments suggests that the main issue responsible for the poor performance of the material is the presence of surface defects in the nanofibres, which are caused by their lack of graphitic character. Therefore, the next step in this project was the investigation of various strategies to improve the crystallinity of the material, with the assumption that more graphitic CNFs would allow an intercalation-based energy storage mechanism to take place, leading to a performance improvement. 


\subsubsection{Efforts to improve nanofibre graphitisation}

Earlier in this chapter it was mentioned that the instrumentation available only allowed heat treatments up to $1400{ }^{\circ} \mathrm{C}$. This posed a great limitation to the amount of graphitic character obtainable from PAN-based CNFs. However, a quick literature review reveals that there are strategies to effectively obtain carbon materials with a higher degree of graphitic character at a low carbonisation temperature: the most common consists of conducting the heating process in the presence of a catalyst compound that facilitates the graphitisation of the material. Metals such as $\mathrm{Fe}, \mathrm{Co}$, and $\mathrm{Ni}$ are most commonly used, ${ }^{41-43}$ but more recent reports also explore the possibility of using different materials, such as carbon nanotubes or boric acid for this purpose. ${ }^{44-46}$ Among the strategies reported, metallic catalysts were chosen, because of their ease of use and low cost.

The literature on this topic is quite broad, revealing a large number of different techniques aimed at the graphitisation of carbon from many different precursors, and using different methods for the addition of the catalyst. ${ }^{45,47-50}$ Based on the previous literature review, none of these processes seem to have been applied to electrospun PAN-derived CNFs. Several methods of catalyst addition were therefore tested, to find out which one would yield the best result on the material. Some of the unsuccessful techniques included soaking the precursor fibre mats in a metal salt solution and using a solvothermal synthesis to make the catalyst grow on the surface of the fibres. Eventually, the best methodology for this purpose proved to be possibly the simplest one: adding a readily soluble metallic salt or coordination complex in the electrospinning solution. Two metal precursors, ferrocene and cobalt (II) acetate tetrahydrate, were used in these attempts and were chosen because of their solubility in DMF and their availability in our lab. Although both metals are initially present in an oxidised state, it was assumed that during the high temperature treatment, the presence of reducing carbon in an inert atmosphere would cause the reduction of the metal ions to their zero-valent state and promote the catalytic graphitisation. It is worth noting that although such compounds are being referred to as "catalysts", the addition of the complexes in catalytic quantities (i.e. less than $5-10 \%$ than the mass of PAN in the solution) did not actually provide any significant results. In fact, the concentrations of metal salt needed to be as high as saturation levels (up to 50\% metal salt/PAN mass ratio) to obtain any significant results. Despite the addition of catalyst compounds to 
the precursor solution, the electrospinning process could be conducted relatively easily, as the fibre mats were obtained using the same experimental parameters used for pure PAN nanofibres. The heat treatment was also maintained, with the carbonisation step set to $1400{ }^{\circ} \mathrm{C}$. After the heat treatment, the CNF mats were rinsed in concentrated aqueous hydrochloric acid several times to remove their metal content.

SEM was then performed on the newly fabricated materials to verify their morphology. It can be seen from Figure 5.16 that the nanofibres show the presence of beads and defects; this is likely caused by the change in the components of precursor solution, and further optimisation of the electrospinning parameters

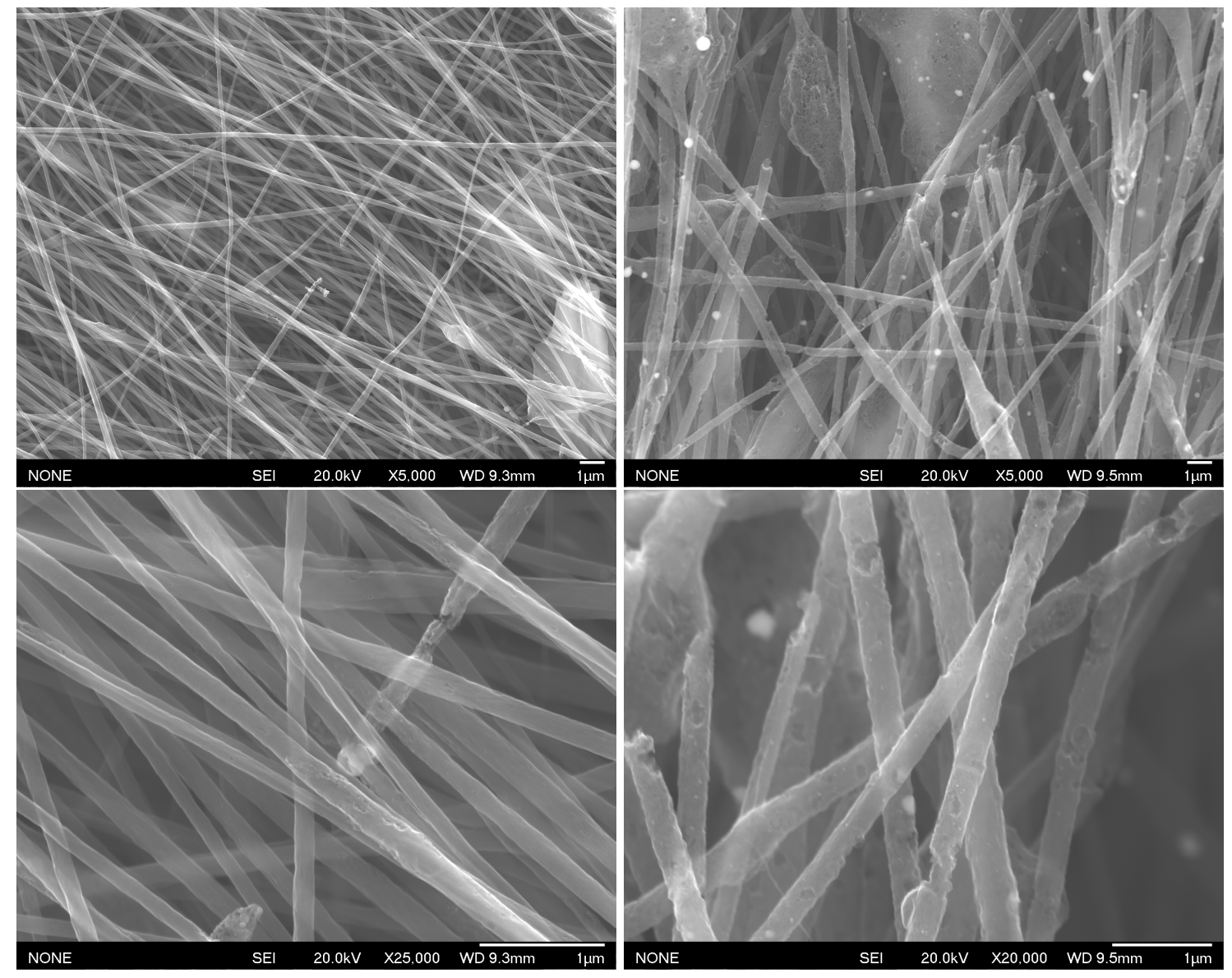

FIGURE 5.16: SEM micrographs at different magnifications (top: $x 5,000$; bottom left: $\times 25,000$; bottom right: $\times 20,000$ ) of carbon nanofibres with enhanced graphitisation through the use of metal catalysts: ferrocene precursor (left) and cobalt acetate precursor (right). 
would be required to remove them. The presence of high-contrast spherical particles is also observed, which are easily identified as residual metal particles. These are embedded in the fibres in such a way that they are not accessible by the acid and therefore could not be removed in the treatment. This is also hinted by the presence of hemispherical cavities in the fibres, which are likely caused by metal particles that used to lie on the surface of the nanofibre and have been etched by the acid treatment. It is also worth noting that the overall concentration of cavities and residual particles is higher in the sample prepared using cobalt acetate than the one prepared using ferrocene. This could possibly indicate that ferrocene is somehow not being properly electrospun in the fibres, and therefore cobalt acetate is a better additive for this purpose, as it allows higher catalyst loading in the fibres.

Figure 5.17 shows the X-ray diffractograms of the CNFs prepared with metal catalysts compared to the fibres prepared from pure PAN nanofibres. It is clear that, with the addition of metal catalysts, the (002) peak becomes notably narrower, and shifts to a slightly higher diffraction angle. This has been demonstrated to correspond to an increase in the size of crystalline domains, and a decrease in the interlayer spacing between the basal planes of graphite, in accordance with Bragg's law (see also Equation 3.1). ${ }^{8}$ It is also worth noting that, when cobalt acetate is used as the catalyst, the diffractogram of the CNFs shows the (002) peak

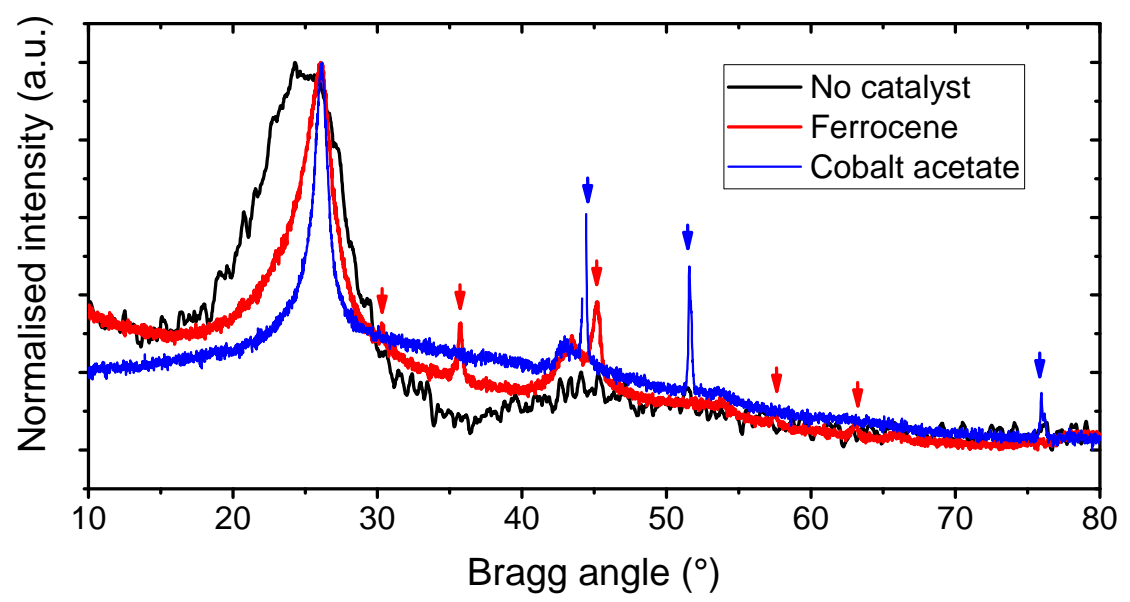

FIGURE 5.17: X-ray diffractograms of CNFs with enhanced graphitisation through the use of metal catalysts. The blue and red marked peaks are relative to residual metallic cobalt and iron oxide impurities in the samples, respectively. 
superimposed on top of a sloped baseline, with a maximum centred around 27 degrees. One possible interpretation of this pattern could be that two distinct phases are present in the material: a highly ordered graphitic phase that generates the sharp (002) peak and an amorphous or turbostratic (a phase of carbon in which the graphene sheets are misaligned, folded, and poorly stacked) phase ${ }^{51}$ that generates the sloped baseline. This morphology could be possibly caused by the coalescence of the catalyst particles, causing localised areas of crystallinity, ${ }^{41}$ as also shown by the SEM pictures in Figure 5.16. Interestingly, this does not seem to be the case for the fibres prepared using the ferrocene catalyst. The diffractogram reveals a sharper (002) peak (albeit broader than the one relative to the cobalt acetate sample), on top of a more traditional-looking baseline. This pattern would likely indicate a material with a single, uniform phase characterised by smaller graphitic domains. This is possibly due to the poor electrospinning performance of the ferrocene-catalysed CNFs, leading to an overall lower concentration of catalyst in the fibres and therefore a lower degree of crystallinity in the final material. Raman spectroscopy was also performed on the materials. Figure 5.18 shows that the addition of metal catalysts causes a reduction in the overall width of the $\mathrm{D}$ and $G$ peaks, located at about 1350 and $1600 \mathrm{~cm}^{-1}$ respectively, indicating an increase in crystallinity of the carbon material. Furthermore, the ratio between such peaks is also decreased, suggesting a reduction of the amount of defects and $s p^{3}$-hybridised carbon atoms. ${ }^{52,53}$ This trend is also consistent with what was observed in the XRD data, as the Raman spectra suggest that cobalt acetate sample shows the greatest enhancement in graphitic character.

The graphitic properties of cobalt acetate-enhancement were also studied using high-resolution TEM (HRTEM). Figure 5.19 shows that the nanofibres are characterised by two different types of domains. In the vicinity of the residual metallic cobalt particles (such as the high-contrast spot in Figures 5.19a and 5.19c), the presence of rod-like domains with a highly ordered crystal structure can be observed (Figures 5.19c, 5.19e). Such areas could plausibly correspond to the enhanced graphitic character, made possible by the inclusion of the metal catalyst. The other region is characterised by circular or curled domains, with an undulated, seemingly less-ordered graphite structure (figures 5.19b, 5.19d), typical of 


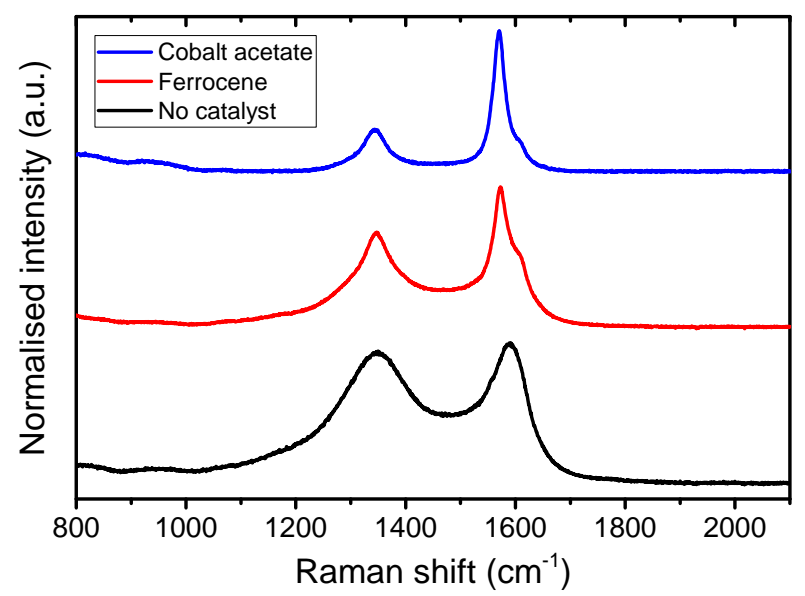

FIGURE 5.18: Raman spectra of CNFs with enhanced graphitisation through the use of metal catalysts.

turbostratic carbon. It is worth noting that neither of these regions are observable in HRTEM images of non-enhanced CNFs, but only uniform regions of disordered carbon can be observed (see also Figure A.14). The presence of two distinct regions with different degrees of crystallinity is consistent with the features observed in the X-ray diffractogram of the material and is also in good agreement with the electron diffraction pattern in Figure 5.19f. The presence of a series of low-resolution diffraction rings can be observed in this pattern, with the narrowest corresponding to the (002) lattice of graphite. Such a low-resolution feature likely corresponds to the diffused areas of turbostratic carbon in the fibres, however, the presence of brighter spots is also observed inside the narrowest ring, which are possibly ascribable to the highly crystalline domains observed in the nanofibres. These brighter spots are unlikely to be related to the cobalt acetate residues, as the diffraction image was acquired from a section of a single nanofibre with no visible metallic cobalt particles present.

Based on the XRD, Raman, and HRTEM data above, it would therefore appear that the addition of an appropriate metal-containing compound in the electrospinning solution can indeed result in an enhancement the graphitic character of CNFs. Of the compounds examined, cobalt acetate appears to be the best candidate for this purpose, as it corresponds to a greater overall enhancement than ferrocene. 


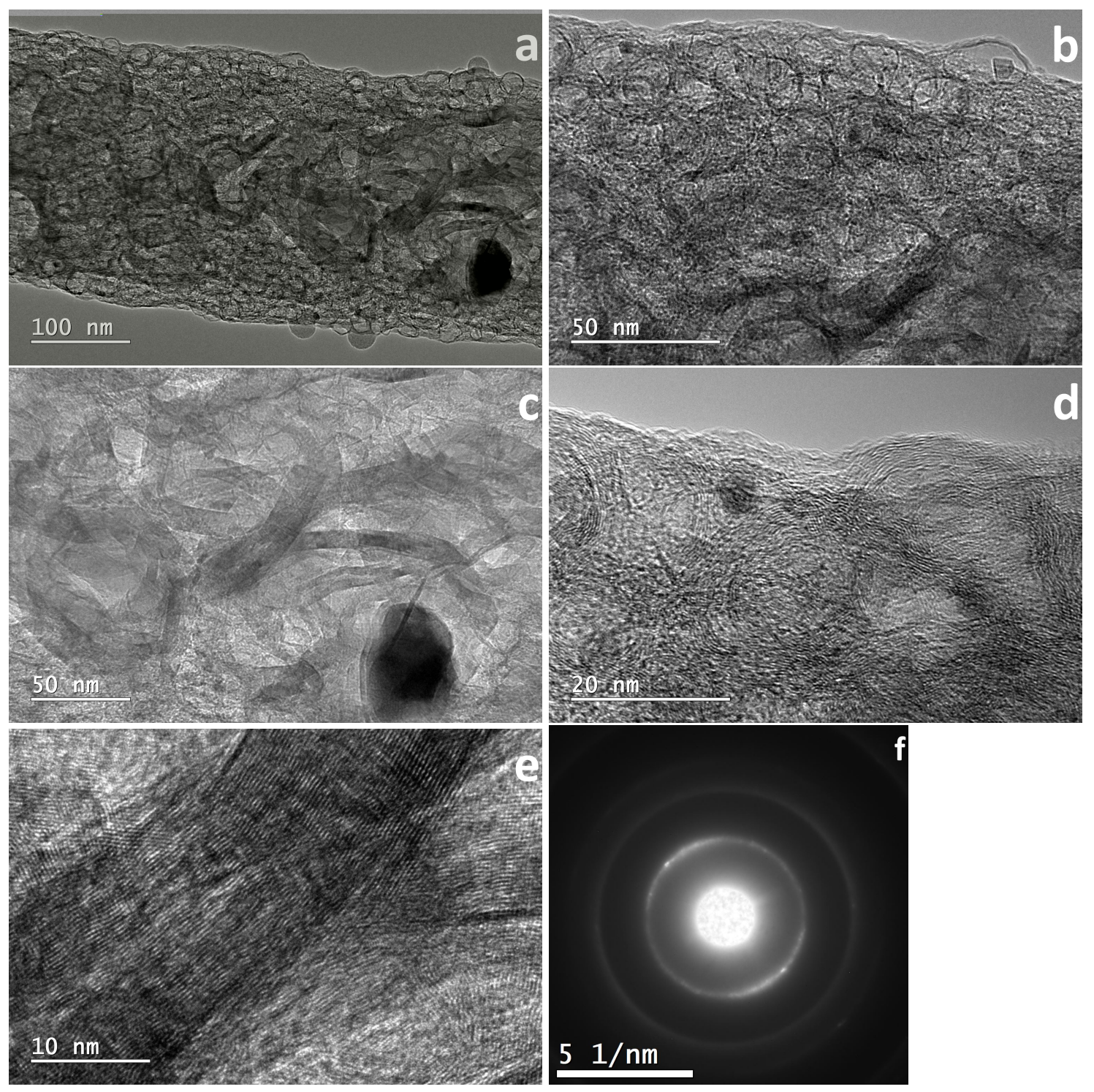

FIGURE 5.19: HRTEM micrographs (a-e) and electron diffraction (f) image of carbon nanofibres prepared using cobalt acetate as metal catalyst precursor.

\subsubsection{Electrochemical tests}

The electrochemical performance of the enhanced materials was tested by assembling devices with the same method described previously. Unfortunately, the enhanced nanofibre mats lost a significant portion of their flexibility and mechanical strength, possibly due to the increased graphitic character and the presence of defects in the fibres left by the metal catalyst particles (as shown in Figure 5.16). This made handling the materials and preparing the devices considerably more 


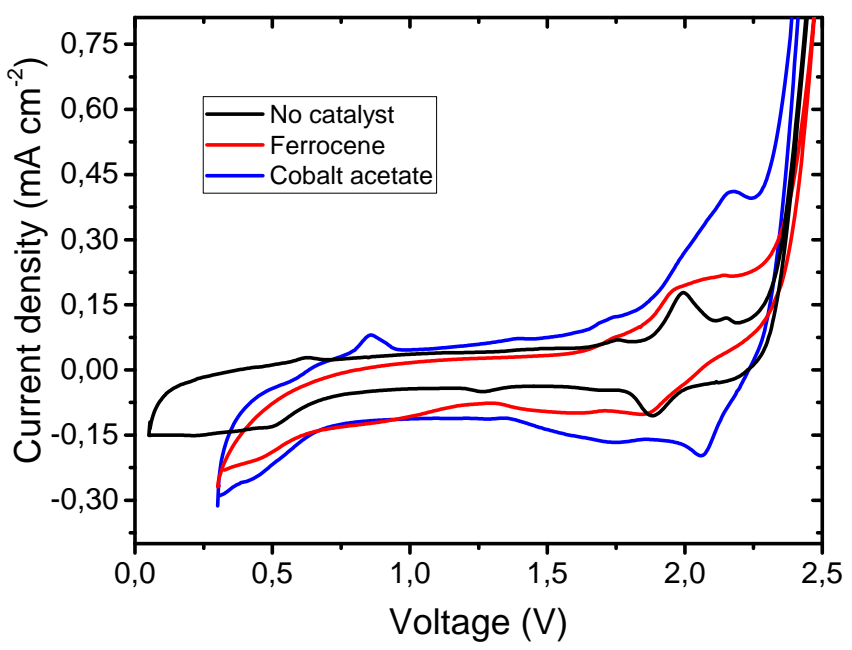

FIGURE 5.20: Cyclic voltammogram (third cycle, $10 \mathrm{mV} \mathrm{s}^{-1}$ ) of Swagelok-type cells built using CNFs, with enhanced graphitisation through the use of metal catalysts, as cathode. The current density is referred to the projected cathode area; the voltage reported in this plot is the cell potential.

challenging; nevertheless, the mats were carefully cut into the appropriately sized cathode discs and used in the devices successfully without further treatments.

Figure 5.20 shows cyclic voltammograms of the devices prepared using the enhanced CNFs as cathodes compared to the fibres prepared from pure PAN nanofibres. It is immediately observed that the current densities are notably higher compared to the previous devices. This could be a consequence of the increased conductivity of the enhanced CNFs. Furthermore, although the hysteretic baseline appears more intense for the enhanced CNFs, the ratio between the intensity of the faradaic and capacitive currents appears to be slightly increased, especially in the case of the sample prepared with cobalt acetate. Similarly to the non-catalysed CNFs, a cluster of oxidation and reduction peaks (albeit less defined) can be observed in the range between 1.7 and $2.3 \mathrm{~V}$ that resemble the ones relative to $\mathrm{AlCl}_{4}^{-}$ intercalation and deintercalation of ions into graphite by previous publications. ${ }^{1,35}$ This could indicate that the slight enhancement in the graphitic character of the nanofibres could allow a larger portion of intercalation-based energy storage. A small oxidation peak centred around about $0.8 \mathrm{~V}$ is also observed in the case of the cobalt acetate CNFs, which could perhaps be related to the oxidation of the residual metallic cobalt particles in the material. Another interesting feature of the enhanced CNF voltammograms is the negative current developing as the voltage 

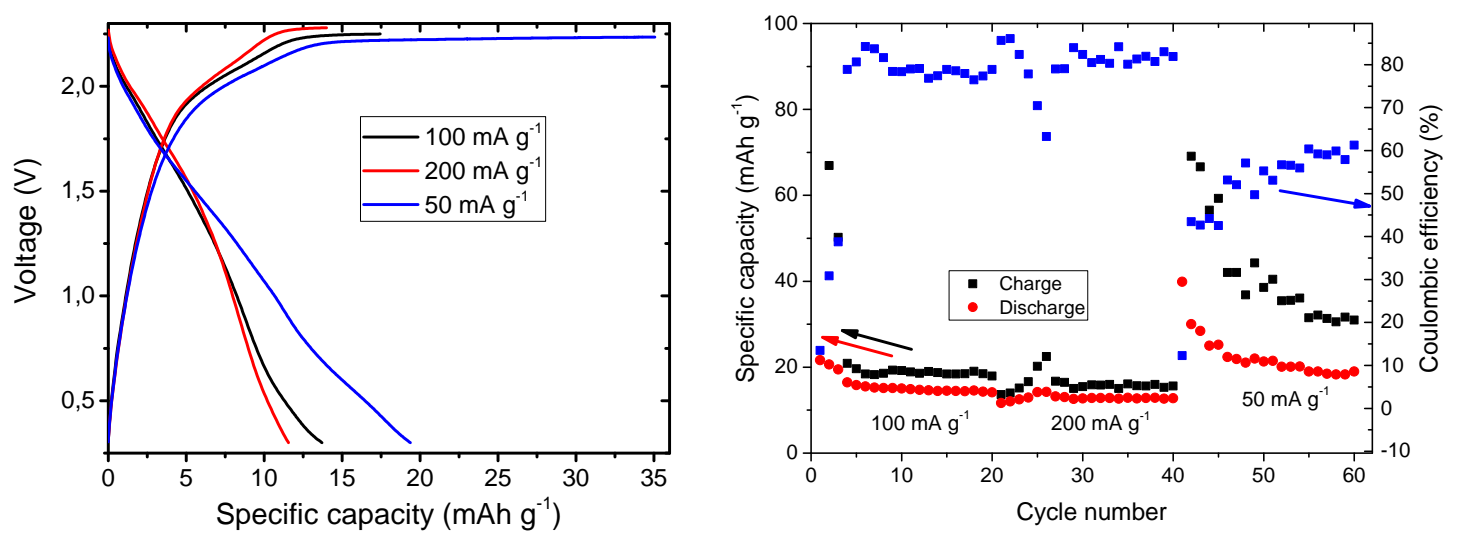

FIGURE 5.21: (Left) Typical galvanostatic charge-discharge profiles (tenth cycle) at various current rates of a Swagelok-type cell built using cobalt acetateenhanced CNFs as cathode. The voltage reported in this plot is the cell potential. (Right) Specific capacities and coulombic efficiencies of a typical galvanostatic charge-discharge test of a Swagelok-type cell built using cobalt acetate-enhanced

CNFs as cathode.

decreases below $0.6 \mathrm{~V}$. This is likely caused by the underpotential deposition of aluminium onto the cathode, ${ }^{54,55}$ which could be promoted by the residual metal particles. Due to this, the voltammograms acquired for the enhanced CNFs had to be acquired with a narrowed voltage window to avoid the plating of aluminium onto the cathode.

Galvanostatic charge-discharge tests were also performed on the devices. Figure 5.21 (left) shows the typical galvanostatic profiles of a device constructed using cobalt acetate-enhanced CNFs. The graph shows that the material yields a behaviour very similar to the non-enhanced CNFs, as no significant discharge plateaus can be observed at any of the measured current rates and specific capacities are low. This indicates that even in this case, no significant intercalation processes take place in the material. A similar behaviour was also observed for the ferrocene-enhanced CNFs (Figure A.15). The early onset of electrolyte decomposition and formation of an SEI as described in Section 5.2.2 is also still present in both materials. Because of this, the upper voltage cutoff needs to be lowered to obtain reasonable coulombic efficiencies. Similarly to the non-enhanced CNFs, the voltage window also needs to be adjusted at each cycling rate to optimise coulombic efficiency and specific discharge capacity. These results suggest that 
the enhancement in graphitic character of the material is still not enough to allow an intercalation-based energy storage mechanism in AIBs, and the material still mainly works through a capacitive or pseudo-capacitive behaviour, leading to poor energy storage performance.

\subsection{Conclusions}

In conclusion, the results outlined in this chapter have demonstrated that CNFs could potentially be used as a nanostructured cathode for AIBs. Their low degree of crystallinity, however, causes them to not withstand any significant intercalation-based energy storage mechanism, and the main energy storage mechanism in the material is limited to capacitive or pseudo-capacitive behaviour, leading to overall poor performance. Furthermore, the unexpected phenomena of electrolyte decomposition at lower voltages and the possible formation of an SEI have been investigated. Experimental evidence from both this project and previous literature reports indicate that this behaviour may be caused by combination of a high surface area and a low degree of crystallinity, which is correlated to the presence of surface defects in the material.

Although the use of metal catalysts can produce an observable enhancement in the graphitic character of the nanofibres, such modification does not appear to be sufficient to yield a noticeable increase in the energy storage performance of the material. This indicates that only highly graphitic materials can work through an intercalation-based energy storage mechanism in AIBs, partially thanks to their low density of surface defects. It is worth noting that even in pre-existing literature reports on "low temperature graphitisation", ${ }^{41-44,46}$ the increase in graphitic character is still modest at temperatures such as the ones accessible by the instrumentation available, and the best results were obtained at temperatures higher than $2000{ }^{\circ} \mathrm{C}$. Therefore, these limitations prohibited the production of a carbon material with the ideal features. The fabrication of highly graphitic CNFs and the study of their performance as cathodes in AIBs, however, could be an interesting research topic to be pursued in the future. 


\subsection{Experimental section}

All the chemicals mentioned in this section were purchased from Sigma-Aldrich and used without any additional treatment, unless otherwise specified. Al foil and pyrolytic graphite paper were purchased from MTI Corp.

SEM micrographs and EDX data were acquired with a JEOL-JSM6500F field emission scanning electron microscope, using an accelerating voltage of $20 \mathrm{kV}$. In order to enhance imaging contrast, polymeric samples were sputter-coated with a $10 \mathrm{~nm}$ Pt layer.

TEM micrographs were acquired with a JEOL-JEM2100F transmission electron microscope, operating at an accelerating voltage of $200 \mathrm{kV}$. Samples were prepared by dispersing the material in ethanol via ultrasonication and drop casting onto a lacey carbon grid.

Thermogravimetric analysis was performed using a Shimadzu TGA-50 thermogravimetric analyser operating under a flow of $50 \mathrm{~mL} / \mathrm{min}$ of either air or nitrogen using the following temperature programme: heating at $10{ }^{\circ} \mathrm{C} /$ minute until $120{ }^{\circ} \mathrm{C}$, hold for 10 minutes to eliminate residual water in the sample, heating at $10^{\circ} \mathrm{C} /$ minute until the final temperature, and hold for another ten minutes.

BET surface area measurements were performed using a Micrometrics FlowSorb II 2300 instrument at a temperature of $77 \mathrm{~K}$ using a $30 \% \mathrm{~N}_{2}$ in He mixture.

$X$-ray diffractograms were acquired using a PANalytical X'pert PRO XRD diffractometer operating in reflection mode, using a $\mathrm{Cu} \mathrm{K} \alpha$ X-ray source, in the Bragg angle range between 10 and 80 degrees. Samples were prepared by lightly grinding the material and depositing on a zero-background sample holder.

Raman spectra were acquired using a LabRAM HR800 Raman spectrometer (Horiba Jobin-Yvon), using an excitation wavelength of $514.5 \mathrm{~nm}$, a grating of $1800 \mathrm{gr} / \mathrm{mm}$, and objective magnification of 100x. The acquisition time was approximately 10 seconds for each spectrum.

XPS spectra were acquired using a Kratos Axis UltraDLD X-ray photoelectron spectrometer (Kratos Analytical, Manchester, UK) equipped with a hemispherical electron energy analyser. Spectra were excited using monochromatic $\mathrm{Al} \mathrm{K}_{\alpha} \mathrm{X}$-rays $(1486.69 \mathrm{eV})$ with the $\mathrm{X}$-ray source operating at $150 \mathrm{~W}$. This instrument illuminates a large area on the surface and then using hybrid magnetic and electrostatic lenses collects photoelectrons from a desired location on the surface. In this case the 
analysis area was a 300 by $700 \mu \mathrm{m}$ spot obtained using the hybrid magnetic and electrostatic lens and the slot aperture. Measurements were carried out in a normal emission geometry. Core level scans were collected with a pass energy of 20 $\mathrm{eV}$. The analysis chamber was at pressures in the $10^{-9}$ torr range throughout the data collection. Data analysis was performed using the CasaXPS software.

Cyclic voltammetry tests were performed using a Metrohm Autolab PGSTAT128N potentiostat operating in a 2-electrode configuration: the cathode terminals of the devices were connected to the working electrode, and the anode terminals were connected to the counter and reference electrode.

Galvanostatic charge-discharge experiments were performed using a NEWARE BTS CT-4008-5V10mA-164 (MTI Corp.) battery analyser system.

For $e x$-situ experiments, the cycled devices were disassembled inside the glovebox, and the cathodes were washed with ethanol to remove any residual electrolyte.

\section{Fabrication of CNFs}

Precursor solutions were prepared by adding $0.8 \mathrm{~mL}$ of PAN (average $\mathrm{M}_{w}$ : $150,000)$ to $10 \mathrm{~mL}$ of DMF under magnetic stirring. For enhanced CNFs, up to 0.8 $\mathrm{g}$ of ferrocene or cobalt acetate tetrahydrate were added to the solution, reaching saturation point. The solutions were then loaded into plastic syringes with a stainless steel, 18-gauge, blunt tip needle. The electrospinning process was then performed, using a voltage of $15 \mathrm{kV}$, a flow rate of $1 \mathrm{~mL} /$ hour, and a tip-to-collector distance of $13 \mathrm{~cm}$. A rotating drum collector was used in the process to optimise the form factor of the nanofibre mats. The resulting fibres were first heat-treated in air using a muffle furnace with the following heating programme: heating at $5{ }^{\circ} \mathrm{C} /$ minute until $220^{\circ} \mathrm{C}$, holding the temperature for 2 hours, and cooling naturally to room temperature. Finally, the fibres were carbonised under a nitrogen atmosphere using a tube furnace with the following heating programme: heating at $4{ }^{\circ} \mathrm{C} /$ minute until $200{ }^{\circ} \mathrm{C}$, hold temperature for 20 minutes, heating at 10 ${ }^{\circ} \mathrm{C} /$ minute until $600{ }^{\circ} \mathrm{C}$, hold temperature for 20 minutes, heating at $5{ }^{\circ} \mathrm{C} /$ minute until $1400{ }^{\circ} \mathrm{C}$, hold temperature for 1 hour, cooling at $5{ }^{\circ} \mathrm{C} /$ minute until $200{ }^{\circ} \mathrm{C}$, and finally cooling naturally to room temperature. 


\section{Fabrication of carbon film}

A precursor solution was prepared by adding $0.8 \mathrm{~mL}$ of PAN (average $\mathrm{M}_{w}$ : $150,000)$ to $10 \mathrm{~mL}$ of DMF under magnetic stirring. Roughly $3 \mathrm{~mL}$ of this solution were then poured into a Petri dish, and the solvent was evaporated in air over 2 hours. The resulting PAN film was first heat-treated in air using a muffle furnace with the following heating programme: heating at $5{ }^{\circ} \mathrm{C} /$ minute until 220 ${ }^{\circ} \mathrm{C}$, holding the temperature for 2 hours, and cooling naturally to room temperature. Finally, the film was carbonised under a nitrogen atmosphere using a tube furnace with the following heating programme: heating at $4{ }^{\circ} \mathrm{C} /$ minute until 200 ${ }^{\circ} \mathrm{C}$, hold temperature for 20 minutes, heating at $10{ }^{\circ} \mathrm{C} /$ minute until $600{ }^{\circ} \mathrm{C}$, hold temperature for 20 minutes, heating at $5{ }^{\circ} \mathrm{C} /$ minute until $1400{ }^{\circ} \mathrm{C}$, hold temperature for 1 hour, cooling at $5{ }^{\circ} \mathrm{C} /$ minute until $200{ }^{\circ} \mathrm{C}$, and finally cooling naturally to room temperature.

\section{Electrolyte preparation}

[EMIm] $\mathrm{Cl}$ and $\mathrm{AlCl}_{3}$ were baked at $100{ }^{\circ} \mathrm{C}$ in a vacuum oven for 48 hours to remove its water content, and immediately brought inside a nitrogen-filled glovebox with the $\mathrm{O}_{2}$ and $\mathrm{H}_{2} \mathrm{O}$ levels kept below $1 \mathrm{ppm}$. The electrolyte was prepared by gradually adding 1.3 equivalents of anhydrous $\mathrm{AlCl}_{3}$ to 1 equivalent [EMIm]Cl under magnetic stirring inside the aforementioned glovebox. An exothermic reaction takes place, after which a brown, slightly viscous liquid is formed.

\section{Device construction}

CNF mats, carbon film, and pyrolytic graphite paper sheets were cut into $11 \mathrm{~mm}$ diameter discs to be used as cathode in the devices. Prototype batteries were then assembled using a custom-built Swagelok-type cell consisting of a cylindrical PEEK casing with an inner diameter of $12 \mathrm{~mm}$ and two Mo rods as the current collectors (see also Section 3.4.2). All the components of the device were first baked at $100{ }^{\circ} \mathrm{C}$ in a vacuum oven for at least 2 hours to remove any residual water, then immediately transferred inside a nitrogen-filled glovebox with the $\mathrm{O}_{2}$ and $\mathrm{H}_{2} \mathrm{O}$ levels kept below $1 \mathrm{ppm}$. One of the previously prepared carbonaceous material discs was used as a cathode, and $11 \mathrm{~mm}$ diameter, high purity $\mathrm{Al}$ foil discs were 
used as the anode. Glass microfibre (GF/D) discs with $12 \mathrm{~mm}$ diameters, soaked in approximately $300 \mu \mathrm{L}$ of electrolyte, were used as separators. The devices were then wrapped with Parafilm as an additional moisture barrier, and taken outside the glovebox for electrochemical testing.

\section{Bibliography}

${ }^{1}$ M.-C. Lin, M. Gong, B. Lu, Y. Wu, D.-Y. Wang, M. Guan, M. Angell, C. Chen, J. Yang, B.-J. Hwang, and H. Dai, "An ultrafast rechargeable aluminium-ion battery," Nature, vol. 520, pp. 324-328, Apr. 2015.

${ }^{2}$ J. V. Rani, V. Kanakaiah, T. Dadmal, M. S. Rao, and S. Bhavanarushi, "Fluorinated Natural Graphite Cathode for Rechargeable Ionic Liquid Based Aluminum-Ion Battery," Journal of The Electrochemical Society, vol. 160, pp. A1781-A1784, Jan. 2013.

${ }^{3}$ S. K. Nataraj, K. S. Yang, and T. M. Aminabhavi, "Polyacrylonitrile-based nanofibers-A stateof-the-art review," Progress in Polymer Science, vol. 37, pp. 487-513, Mar. 2012.

${ }^{4}$ M. Inagaki, Y. Yang, and F. Kang, “Carbon Nanofibers Prepared via Electrospinning," Advanced Materials, vol. 24, pp. 2547-2566, May 2012.

${ }^{5}$ Y. Bai, Z.-H. Huang, Z.-X. Zhang, and F. Kang, "Improvement of the hydrophilicity of electrospun porous carbon nanofibers by grafting phenylsulfonic acid groups," Journal of Colloid and Interface Science, vol. 394, pp. 177-182, Mar. 2013.

${ }^{6}$ M. S. A. Rahaman, A. F. Ismail, and A. Mustafa, "A review of heat treatment on polyacrylonitrile fiber," Polymer Degradation and Stability, vol. 92, pp. 1421-1432, Aug. 2007.

${ }^{7}$ C.-K. Liu, K. Lai, W. Liu, M. Yao, and R.-J. Sun, "Preparation of carbon nanofibres through electrospinning and thermal treatment," Polymer International, vol. 58, no. 12, pp. 1341-1349, 2009.

${ }^{8}$ A. Ramos, I. Cameán, and A. B. García, "Graphitization thermal treatment of carbon nanofibers," Carbon, vol. 59, pp. 2-32, Aug. 2013.

${ }^{9}$ A. Oberlin, "Carbonization and graphitization," Carbon, vol. 22, pp. 521-541, Jan. 1984.

${ }^{10}$ P. Wang, D. Zhang, F. Ma, Y. Ou, Q. N. Chen, S. Xie, and J. Li, “Mesoporous carbon nanofibers with a high surface area electrospun from thermoplastic polyvinylpyrrolidone," Nanoscale, vol. 4, pp. 7199-7204, Oct. 2012.

${ }^{11}$ M. Gauthier, T. J. Carney, A. Grimaud, L. Giordano, N. Pour, H.-H. Chang, D. P. Fenning, S. F. Lux, O. Paschos, C. Bauer, F. Maglia, S. Lupart, P. Lamp, and Y. Shao-Horn, "Electrode-Electrolyte Interface in Li-Ion Batteries: Current Understanding and New Insights," The Journal of Physical Chemistry Letters, vol. 6, pp. 4653-4672, Nov. 2015.

${ }^{12}$ G. Antonio Elia, I. Hasa, G. Greco, T. Diemant, K. Marquardt, K. Hoeppner, R. Jürgen Behm, A. Hoell, S. Passerini, and R. Hahn, "Insights into the reversibility of aluminum graphite batteries," Journal of Materials Chemistry A, vol. 5, no. 20, pp. 9682-9690, 2017.

${ }^{13}$ G. Greco, D. Tatchev, A. Hoell, M. Krumrey, S. Raoux, R. Hahn, and G. Antonio Elia, “Influence of the electrode nano/microstructure on the electrochemical properties of graphite in aluminum batteries," Journal of Materials Chemistry A, vol. 6, no. 45, pp. 22673-22680, 2018. 
${ }^{14}$ G. A. Elia, J.-B. Ducros, D. Sotta, V. Delhorbe, A. Brun, K. Marquardt, and R. Hahn, “Polyacrylonitrile Separator for High-Performance Aluminum Batteries with Improved Interface Stability," ACS Applied Materials \& Interfaces, vol. 9, pp. 38381-38389, Nov. 2017.

${ }^{15}$ D.-Y. Wang, C.-Y. Wei, M.-C. Lin, C.-J. Pan, H.-L. Chou, H.-A. Chen, M. Gong, Y. Wu, C. Yuan, M. Angell, Y.-J. Hsieh, Y.-H. Chen, C.-Y. Wen, C.-W. Chen, B.-J. Hwang, C.-C. Chen, and H. Dai, "Advanced rechargeable aluminium ion battery with a high-quality natural graphite cathode," Nature Communications, vol. 8, p. 14283, Feb. 2017.

${ }^{16}$ K. V. Kravchyk, S. Wang, L. Piveteau, and M. V. Kovalenko, "Efficient Aluminum Chloride-Natural Graphite Battery," Chemistry of Materials, vol. 29, pp. 4484-4492, May 2017.

${ }^{17}$ N. P. Stadie, S. Wang, K. V. Kravchyk, and M. V. Kovalenko, "Zeolite-Templated Carbon as an Ordered Microporous Electrode for Aluminum Batteries," ACS Nano, vol. 11, pp. 1911-1919, Feb. 2017.

${ }^{18}$ D. S. Su, S. Perathoner, and G. Centi, "Nanocarbons for the Development of Advanced Catalysts," Chemical Reviews, vol. 113, pp. 5782-5816, Aug. 2013.

${ }^{19}$ X. Mao, G. C. Rutledge, and T. A. Hatton, "Nanocarbon-based electrochemical systems for sensing, electrocatalysis, and energy storage," Nano Today, vol. 9, pp. 405-432, Aug. 2014.

${ }^{20}$ T. Placke, V. Siozios, R. Schmitz, S. F. Lux, P. Bieker, C. Colle, H. W. Meyer, S. Passerini, and M. Winter, "Influence of graphite surface modifications on the ratio of basal plane to "non-basal plane" surface area and on the anode performance in lithium ion batteries," Journal of Power Sources, vol. 200, pp. 83-91, Feb. 2012.

${ }^{21}$ Z. Chen, W. Ren, L. Gao, B. Liu, S. Pei, and H.-M. Cheng, "Three-dimensional flexible and conductive interconnected graphene networks grown by chemical vapour deposition," Nature Materials, vol. 10, p. 424, Apr. 2011.

${ }^{22}$ P. Mérel, M. Tabbal, M. Chaker, S. Moisa, and J. Margot, “Direct evaluation of the sp3 content in diamond-like-carbon films by XPS," Applied Surface Science, vol. 136, pp. 105-110, Oct. 1998.

${ }^{23}$ J. Filik, P. May, S. Pearce, R. Wild, and K. Hallam, "XPS and laser Raman analysis of hydrogenated amorphous carbon films," 13th European Conference on Diamond, Diamond-Like Materials, Carbon Nanotubes, Nitrides and Silicon Carbide, vol. 12, pp. 974-978, Mar. 2003.

${ }^{24}$ J. F. Moulder, W. F. Stickle, P. E. Sobol, and K. D. Bomben, Handbook of X Ray Photoelectron Spectroscopy. Eden Prairie, Minn: Physical Electronics, Feb. 1993.

${ }^{25}$ J.-H. Zhou, Z.-J. Sui, J. Zhu, P. Li, D. Chen, Y.-C. Dai, and W.-K. Yuan, “Characterization of surface oxygen complexes on carbon nanofibers by TPD, XPS and FT-IR," Carbon, vol. 45, pp. 785796, Apr. 2007.

${ }^{26}$ G. Beamson and D. Briggs, High Resolution XPS of Organic Polymers: The Scienta ESCA300 Database. Chichester England ; New York: Wiley, 1st edition ed., Nov. 1992.

${ }^{27}$ S. Caporali, U. Bardi, and A. Lavacchi, "X-ray photoelectron spectroscopy and low energy ion scattering studies on 1-buthyl-3-methyl-imidazolium bis(trifluoromethane) sulfonimide," Journal of Electron Spectroscopy and Related Phenomena, vol. 151, pp. 4-8, Mar. 2006.

${ }^{28}$ V. Lockett, R. Sedev, C. Bassell, and J. Ralston, "Angle-resolved X-ray photoelectron spectroscopy of the surface of imidazolium ionic liquids," Physical Chemistry Chemical Physics, vol. 10, no. 9, pp. 1330-1335, 2008.

${ }^{29}$ D. Weingarth, I. Czekaj, Z. Fei, A. Foelske-Schmitz, P. J. Dyson, A. Wokaun, and R. Kötz, “Electrochemical Stability of Imidazolium Based Ionic Liquids Containing Cyano Groups in the Anion: A Cyclic Voltammetry, XPS and DFT Study," Journal of The Electrochemical Society, vol. 159, pp. H611-H615, Jan. 2012. 
${ }^{30}$ C. Powell, “X-ray Photoelectron Spectroscopy Database XPS,Version 4.1, NIST Standard Reference Database 20," 1989. type: dataset.

${ }^{31}$ R. T. Carlin, J. Fuller, W. K. Kuhn, M. J. Lysaght, and P. C. Trulove, “Electrochemistry of roomtemperature chloroaluminate molten salts at graphitic and nongraphitic electrodes," Journal of Applied Electrochemistry, vol. 26, pp. 1147-1160, Nov. 1996.

${ }^{32}$ T. Jiang, M. J. Chollier Brym, G. Dubé, A. Lasia, and G. M. Brisard, “Electrodeposition of aluminium from ionic liquids: Part I-electrodeposition and surface morphology of aluminium from aluminium chloride (AlCl3)-1-ethyl-3-methylimidazolium chloride ([EMIm]Cl) ionic liquids," Surface and Coatings Technology, vol. 201, pp. 1-9, Sept. 2006.

${ }^{33}$ R. Lungwitz and S. Spange, "Determination of Hydrogen-Bond-Accepting and -Donating Abilities of Ionic Liquids with Halogeno Complex Anions by Means of 1H NMR Spectroscopy," ChemPhysChem, vol. 13, pp. 1910-1916, May 2012.

${ }^{34}$ A. W. Taylor, S. Men, C. J. Clarke, and P. Licence, "Acidity and basicity of halometallate-based ionic liquids from X-ray photoelectron spectroscopy," RSC Advances, vol. 3, no. 24, pp. 94369445, 2013.

${ }^{35}$ A. S. Childress, P. Parajuli, J. Zhu, R. Podila, and A. M. Rao, "A Raman spectroscopic study of graphene cathodes in high-performance aluminum ion batteries," Nano Energy, vol. 39, pp. 6976, Sept. 2017.

${ }^{36}$ P. Bhauriyal, A. Mahata, and B. Pathak, "The staging mechanism of AlCl4- intercalation in a graphite electrode for an aluminium-ion battery," Physical Chemistry Chemical Physics, vol. 19, pp. 7980-7989, Mar. 2017.

${ }^{37}$ L. Zhang, L. Chen, H. Luo, X. Zhou, and Z. Liu, “Large-Sized Few-Layer Graphene Enables an Ultrafast and Long-Life Aluminum-Ion Battery," Advanced Energy Materials, vol. 7, p. 1700034, Aug. 2017.

${ }^{38}$ B. E. Conway, "Transition from "Supercapacitor" to "Battery" Behavior in Electrochemical Energy Storage," Journal of The Electrochemical Society, vol. 138, pp. 1539-1548, Jan. 1991.

${ }^{39}$ A. S. Aricò, P. Bruce, B. Scrosati, J.-M. Tarascon, and W. van Schalkwijk, “Nanostructured materials for advanced energy conversion and storage devices," Nature Materials, vol. 4, pp. 366-377, May 2005.

${ }^{40}$ S. Wang, K. Kravchyk, A. Filippin, U. Müller, A. Tiwari, S. Buecheler, M. Bodnarchuk, and M. Kovalenko, "Aluminum Chloride-Graphite Batteries with Flexible Current Collectors Prepared from Earth-Abundant Elements," Advanced Science, vol. 5, no. 4, 2018.

${ }^{41}$ A. Ōya and H. Marsh, "Phenomena of catalytic graphitization," Journal of Materials Science, vol. 17, pp. 309-322, Feb. 1982.

${ }^{42}$ R. Anton, O. Reetz, and A. A. Schmidt, "In Situ TEM Investigation of Processes Catalyzed by Pd-Ni Alloy Particles on Carbon Substrates in the Presence and Absence of Oxygen," Journal of Catalysis, vol. 149, pp. 474-485, Oct. 1994.

${ }^{43}$ O. P. Krivoruchko and V. I. Zaikovskii, "A new phenomenon involving the formation of liquid mobile metal-carbon particles in the low-temperature catalytic graphitisation of amorphous carbon by metallic Fe, Co and Ni," Mendeleev Communications, vol. 8, pp. 97-99, Jan. 1998.

${ }^{44}$ H. G. Chae, M. L. Minus, A. Rasheed, and S. Kumar, "Stabilization and carbonization of gel spun polyacrylonitrile/single wall carbon nanotube composite fibers," Polymer, vol. 48, pp. 37813789, June 2007. 
${ }^{45}$ Y. Wen, Y. Lu, H. Xiao, and X. Qin, "Further investigation on boric acid catalytic graphitization of polyacrylonitrile carbon fibers: Mechanism and mechanical properties," Materials $\mathcal{E}$ Design (1980-2015), vol. 36, pp. 728-734, Apr. 2012.

${ }^{46}$ Y. Zhang, N. Tajaddod, K. Song, and M. L. Minus, "Low temperature graphitization of interphase polyacrylonitrile (PAN)," Carbon, vol. 91, pp. 479-493, Sept. 2015.

${ }^{47}$ M. Sevilla and A. B. Fuertes, "Catalytic graphitization of templated mesoporous carbons," Carbon, vol. 44, pp. 468-474, Mar. 2006.

${ }^{48}$ M. Kruk, K. M. Kohlhaas, B. Dufour, E. B. Celer, M. Jaroniec, K. Matyjaszewski, R. S. Ruoff, and T. Kowalewski, "Partially graphitic, high-surface-area mesoporous carbons from polyacrylonitrile templated by ordered and disordered mesoporous silicas," Microporous and Mesoporous Materials, vol. 102, pp. 178-187, May 2007.

${ }^{49}$ F. Zhang, D. He, S. Ge, and Q. Cai, "Effect of fiber splitting on the catalytic graphitization of electroless Ni-B-coated polyacrylonitrile-based carbon fibers," Surface and Coatings Technology, vol. 203, pp. 99-103, Oct. 2008.

${ }^{50}$ I. K. Moon, J. Lee, R. S. Ruoff, and H. Lee, "Reduced graphene oxide by chemical graphitization," Nature Communications, vol. 1, p. 73, Sept. 2010.

${ }^{51}$ J. J. Kipling, J. N. Sherwood, P. V. Shooter, and N. R. Thompson, "Factors influencing the graphitization of polymer carbons," Carbon, vol. 1, pp. 315-320, Apr. 1964.

${ }^{52}$ A. C. Ferrari, "Raman spectroscopy of graphene and graphite: Disorder, electron-phonon coupling, doping and nonadiabatic effects," Exploring graphene, vol. 143, pp. 47-57, July 2007.

${ }^{53}$ L. Malard, M. Pimenta, G. Dresselhaus, and M. Dresselhaus, "Raman spectroscopy in graphene," Physics Reports, vol. 473, pp. 51-87, Apr. 2009.

${ }^{54}$ D. M. Kolb, M. Przasnyski, and H. Gerischer, "Underpotential deposition of metals and work function differences," Journal of Electroanalytical Chemistry and Interfacial Electrochemistry, vol. 54, pp. 25-38, July 1974.

${ }^{55}$ P. K. Lai and M. Skyllas-Kazacos, "Aluminium deposition and dissolution in aluminium chloride-n-butylpyridinium chloride melts," Electrochimica Acta, vol. 32, pp. 1443-1449, Oct. 1987. 
Chapter 6

Cathode materials: core-sheath $\mathbf{C} / \mathbf{V}_{2} \mathbf{O}_{5}$ nanofibres 



\subsection{Preface}

The following chapter will describe the fabrication of a nanofibrous material consisting of a conductive carbon core and an external layer made of vanadium oxide. This project was undertaken as a means to combine the knowledge gathered from the fabrication of $\mathrm{V}_{2} \mathrm{O}_{5} \mathrm{NFs}$ (see also Chapter 4) and CNFs (see also Chapter 5) to create a novel material for high-performance AIBs. Looking at pre-existing literature, there is only one report which comes close to using a similar concept for the fabrication of a $\mathrm{C} / \mathrm{V}_{2} \mathrm{O}_{5}$ cathode material: the publication by Chiku et al. ${ }^{1}$ describes an amorphous vanadium oxide/carbon compound, which achieved remarkable performance. The rationale behind this project is that the use of CNFs as the core material could present two advantages: 1) the good mechanical properties of CNFs could theoretically allow the material to be used as a self-standing electrode, removing the need for electrode fabrication, and 2) their good conductivity and directionality of the nanofibres would allow efficient charge transport, overall contributing to improved electrode performance. Therefore, $\mathrm{CNFs}$ would effectively serve as a conducting "scaffold", enhancing the performance of $\mathrm{V}_{2} \mathrm{O}_{5}$, which would act as the primary active material.

\subsection{Core-sheath nanofibre fabrication methods}

The fabrication of core-sheath nanofibres can be achieved in a variety of methods, which have an impact on the final morphology of the material. Different strategies were tested with varying degrees of success. The following section will describe the main efforts attempted in this project.

\subsubsection{Coaxial electrospinning}

An initial attempt to fabricate the material was made by using using coaxial electrospinning (see also Section 3.2.1), as it was hypothesised that using this technique would be the most likely to result in an effectively continuous and uniform core-sheath structure. There are, however, a few potential issues linked with this method: primarily, the number of parameters needing to be optimised dramatically increases when two precursor solutions are spun through a single needle 
at the same time. Furthermore, in order to simultaneously create a nanomaterial containing CNFs and vanadium oxide in close proximity, a series of potentially incompatible heat treatments would need to be performed. In particular, the calcination of $\mathrm{V}_{2} \mathrm{O}_{5}$ requires an oxidising atmosphere (such as air) while the carbonisation step needs to be done in an inert environment to prevent the precursor polymer oxidising into carbon dioxide. In addition, the inclusion of a carrier polymer in the "sheath" precursor solution would also likely be necessary for the electrospinning process to work, and its subsequent removal would then need to be performed. Therefore, in order to obtain a material which contains carbon exclusively at the core, it would likely be necessary to perform a heat treatment that can selectively remove the carrier polymer from the sheath section of the fibres, while leaving the core intact. This is definitely a challenging task, as it would require fine tuning the conditions of the heat treatment.

Despite the challenges posed by the technique, it was decided to nevertheless attempt to fabricate core-sheath nanofibres using this method. In designing the experiment, PAN was chosen for the core of the fibres, due to its ease of use, and the previous experience acquired in the fabrication of CNFs using the polymer. For the sheath, a mixture of PVP and $\mathrm{VO}(\mathrm{acac})_{2}$ was used as the precursor solution. Conventional sol-gel precursors such as VOTIP were purposely avoided, to limit further complications due to the hydrolysis process and clogging of the spinneret.

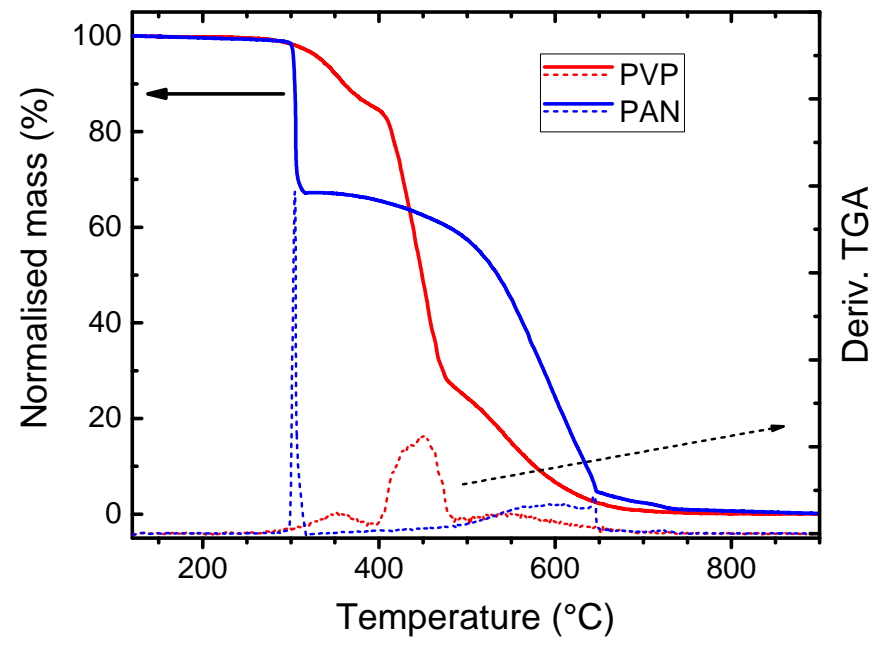

FIGURE 6.1: Comparison of the thermogravimetric profiles (in air atmosphere) of PAN and PVP. 
Poly(methyl methacrylate), PMMA, was initially tested as a carrier polymer for the sheath layer thanks to its lower decomposition temperature. ${ }^{2}$ Unfortunately, no nanofibres could be obtained using this solution, and the idea was therefore discarded. Although PVP is not the best option as a carrier polymer due to its relatively high decomposition temperature in air (as shown by the TGA data in Figure 6.1), previous experiments (briefly mentioned in Section 5.2.1) proved that PVP decomposes almost quantitatively and most of its nanomorphology is disrupted when undergoing the stabilisation and carbonisation steps necessary for the preparation of PAN-based CNFs. Because of this, it was hypothesised that the thermal processes required to convert PAN to pure carbon would at the same time remove the majority of the PVP from the sheath layer.

The electrospinning process proved to be as challenging as predicted, as the concentrations and flow rates of both precursor solutions, along with the other usual operational parameters, needed to be optimised to obtain nanofibres reliably. After a number of attempts, some nanofibres could be finally obtained. The nanomorphology of the best results for this step are displayed in Figure 6.2. The SEM images shows that the nanofibres have a large size distribution, and present numerous bead-shaped defects and agglomeration points. It is unclear whether the core-sheath structure was actually achieved for these nanofibres, as

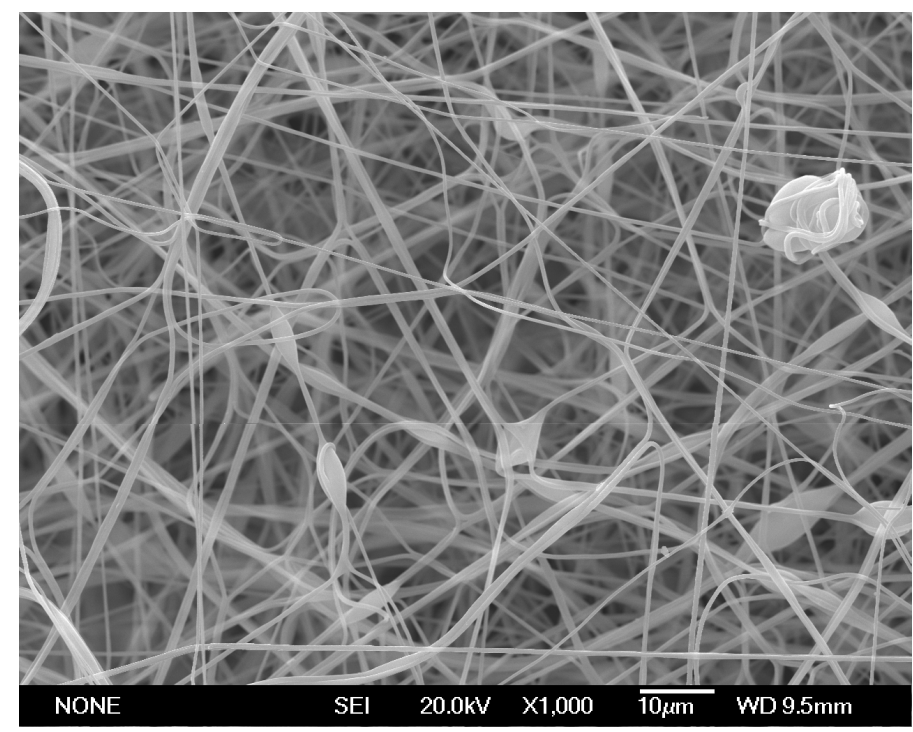

FIGURE 6.2: SEM micrograph of the precursor core-sheath nanofibres prepared using coaxial electrospinning. 
not enough information is provided by the SEM micrograph. Nevertheless, the heat treatments required to convert this material to pure $\mathrm{C}$ and $\mathrm{V}_{2} \mathrm{O}_{5}$ also proved to be quite challenging, as the mats would shrink into a pellet and the nanofibrous morphology would be lost after the stabilisation treatment. This could be caused by a number of reasons such as the melting of any of the precursor components, the poor morphology of the nanofibres, or unwanted side reactions between the precursors. Despite our best attempts, the target material morphology could not be obtained using this technique and new fabrication methods were explored.

\subsubsection{Dip-coated CNF mats}

Due to the many issues encountered using a complex fabrication method such as coaxial electrospinning, a more streamlined approach was attempted based on sol-gel dip-coating. ${ }^{3}$ In this technique, a substrate is dipped in a solution containing the sol-gel precursor. As the substrate gets extracted from the solution and contacts the atmosphere, the thin film of solution coating surface will gradually react with the moisture in the air to form a gel, progressively hardening and forming a solid coat. An annealing step can later also be performed to transform the amorphous coating to a crystalline phase. It was theorised that, although a CNF mat is not a flat substrate, the sol would still be able to coat the fibres uniformly, and form a vanadium-containing sheath that could be later converted to $\mathrm{V}_{2} \mathrm{O}_{5}$ through a thermal annealing step. First, CNF mats were prepared using the same procedure mentioned in Chapter 5 . Then a vanadium solution made of VOTIP, isopropanol, and a small quantity of water was prepared, serving as the sol. The fibres were then manually dipped into the sol, and slowly extracted. This technique proved to be more successful than the previous strategy: SEM imaging revealed that a uniform a thin film of amorphous vanadium oxide coating the nanofibres gel was effectively achieved (Figure 6.3). A certain degree of heterogeneity was observed in the fibres, especially around the edges of the mats, due to the sol accumulating and the solvent evaporating more slowly. This was solved by simply removing such heterogeneous portions from the nanofibre mats.

Following the coating of the CNFs with the vanadium oxide gel, an annealing step was performed to convert the amorphous sheath material to crystalline $\mathrm{V}_{2} \mathrm{O}_{5}$. This 

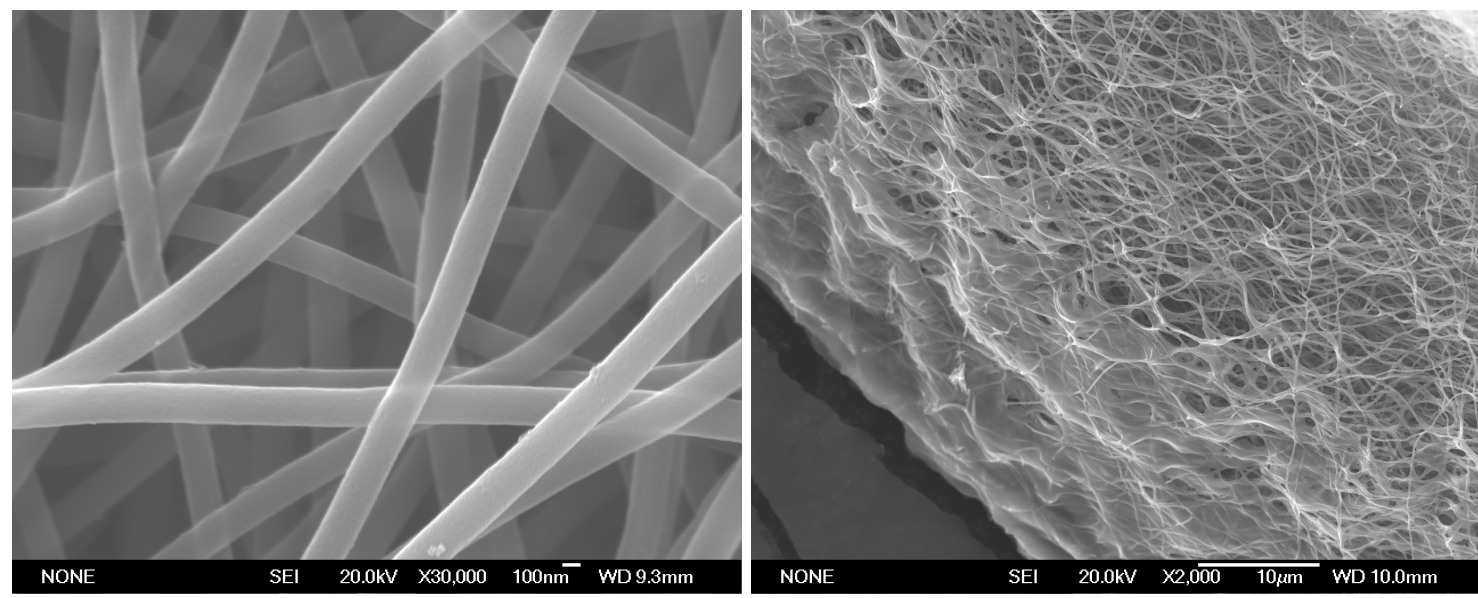

FIGURE 6.3: SEM micrographs of carbon nanofibres which were dip-coated in a vanadium-containing sol to form a $\mathrm{V}_{2} \mathrm{O}_{5}$ sheath layer, showing the difference in coating between the centre (left) and the edges (right) of the fibre mats.

process, however, proved once again to be quite challenging. Initially, the nanofibres were tentatively annealed by heating them to $400{ }^{\circ} \mathrm{C}$ in air, with the reasoning that the vanadium-containing sheath layer would convert to crystalline $\mathrm{V}_{2} \mathrm{O}_{5}$ while the CNFs should not be affected, based on previous TGA measurements (Figure 5.5). After the heat treatment, the material turned a bright yellow colour, likely indicating the successful generation of $\mathrm{V}_{2} \mathrm{O}_{5}$. However, the high brittleness of the sample suggested that the carbon core was likely destroyed in the process. Scanning tunnel electron microscopy-energy dispersive $X$-ray spectroscopy (STEM-EDX) elemental mapping was performed on the sample, confirming that the carbon core was removed during the process, leaving only hollow $\mathrm{V}_{2} \mathrm{O}_{5}$ "nanotubes" in the material (Figure 6.4). This result is unexpected, as the carbon core was thought to remain stable at temperatures as high as $500-550^{\circ} \mathrm{C}$, based on the
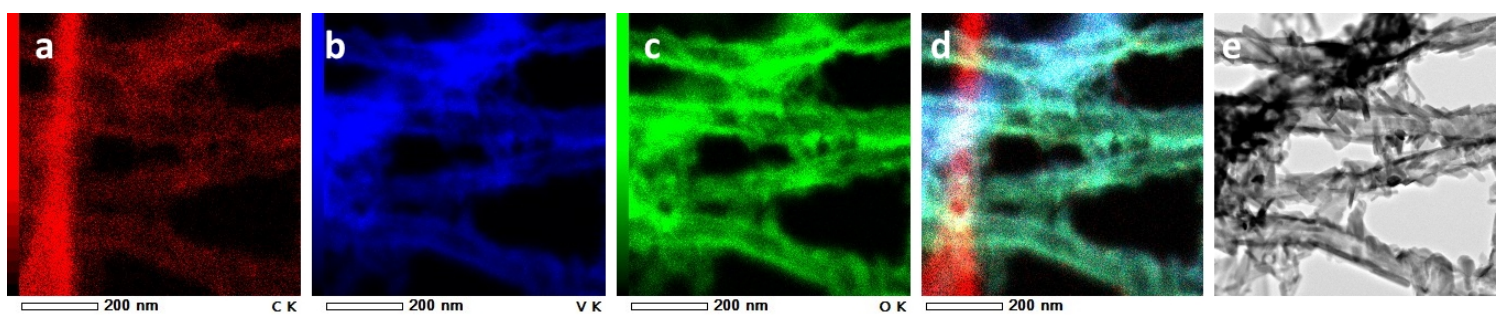

FIGURE 6.4: STEM-EDX elemental maps of the dip-coated carbon nanofibres after annealing in air: carbon (a), vanadium (b), oxygen (c), and overlay (d) maps. (e):

STEM-BF image of the corresponding region. 
previous TGA measurement (Figure 5.5). A possible explanation for this phenomenon could be that the $\mathrm{V}_{2} \mathrm{O}_{5}$ sheath layer acts as a catalyst, promoting the oxidation of CNFs at lower temperatures.

Following the results of the previous tests, a different annealing step was attempted: this time, the dip-coated nanofibres were heated at $400{ }^{\circ} \mathrm{C}$ under an inert $\mathrm{N}_{2}$ atmosphere, to avoid any oxidation process happening to the carbon core. Then, STEM-EDX mapping was performed on the nanofibres to assess the successful formation of a core-sheath structure. It can be seen from Figure 6.5 that vanadium and oxygen are present on the outer layer of the nanofibres, while carbon is still definitely present, and mostly confined to the inner, "core" layer of the fibres. The data therefore suggests that this time the heat treatment did not destroy the carbon core.
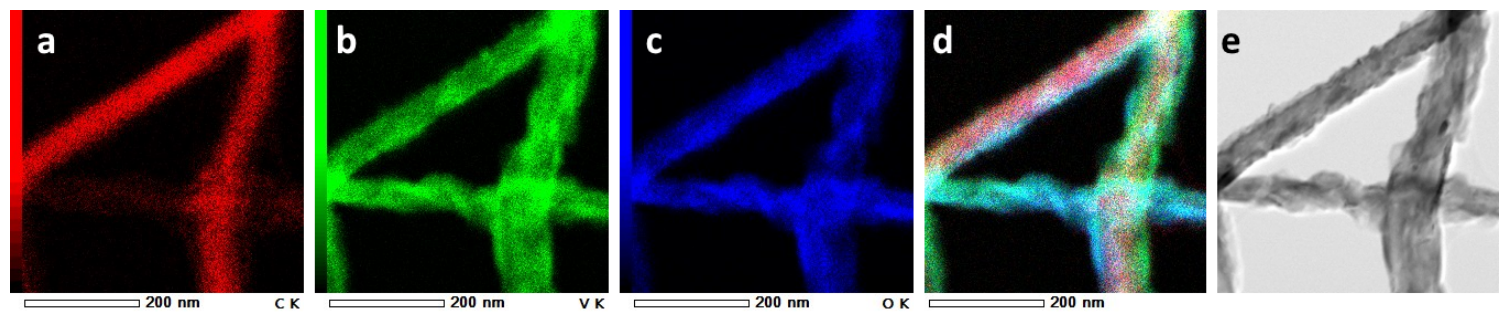

FIGURE 6.5: STEM-EDX elemental maps of the dip-coated carbon nanofibres after annealing under a $\mathrm{N}_{2}$ atmosphere: carbon (a), vanadium (b), oxygen (c), and overlay (d) maps. (e): STEM-BF image of the corresponding region.

$\mathrm{XRD}$ was then performed on the product to elucidate the crystal phase of the product (Figure 6.6). The diffractogram shows the familiar broadened (002) graphite peak, relative to the carbon core, and a series of low-intensity peaks, indicating an overall low degree of crystallinity, with a pattern which does not appear to accurately match any reference file that we could access. The best matching structures were found to belong to vanadium (IV) oxide, $\mathrm{VO}_{2}$.

A possible explanation for this is that during annealing process, the carbonaceous component of the material could have promoted the reduction of vanadium oxide, lowering the oxidation stage of vanadium from $5+$ to $4+$. This hypothesis could also be the reason for the complete removal of the carbonaceous component at such low temperatures in the previous experiment: the carbon core might have been oxidised by the vanadium oxide, which in turn could have been oxidised by the atmospheric oxygen. In this way, the vanadium oxide would de facto act as a catalyst for the oxidation of carbon. Conversely, in an inert atmosphere, $\mathrm{VO}_{2}$ 


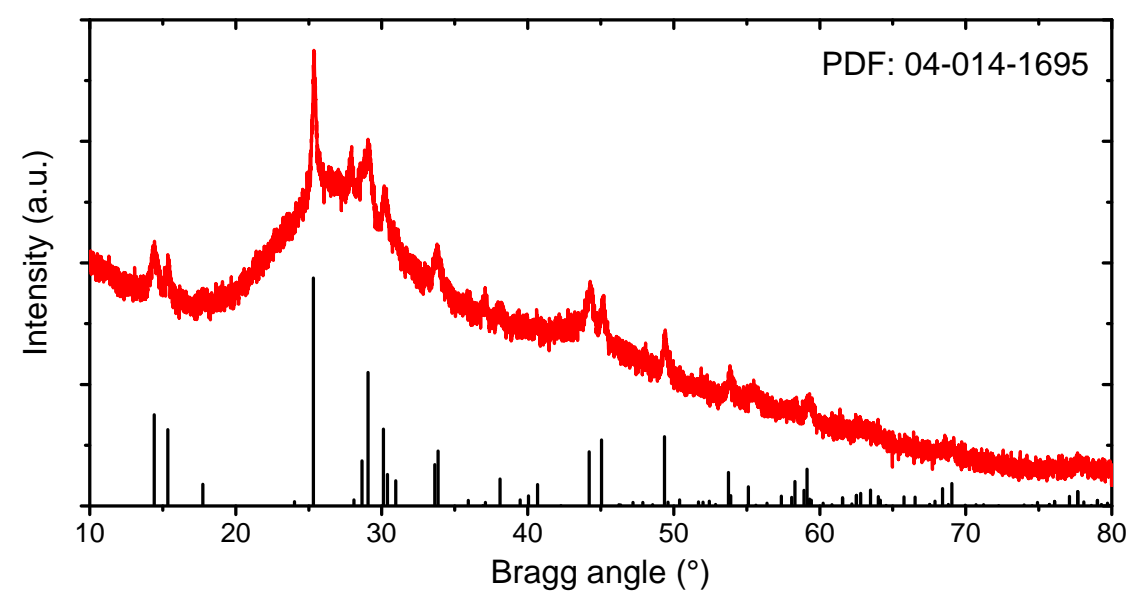

FIGURE 6.6: X-ray diffractograms of the dip-coated carbon nanofibres, annealed under $\mathrm{N}_{2}$ atmosphere, overlaid with the matching ICDD pattern for $\mathrm{VO}_{2}$. The very broad peak centred around $26^{\circ}$ is imputable to the carbon core.

would not be able to convert back to $\mathrm{V}_{2} \mathrm{O}_{5}$, and this catalytic process would not take place. Although a $\mathrm{VO}_{2}$ sheath was not the desired result, previous literature has shown that $\mathrm{VO}_{2}$ also has some activity as a cathode material in AIBs. ${ }^{4}$ Therefore, future studies could be useful to test whether this material could function as well.

\subsubsection{Solvothermal growth on CNFs}

In addition to the aforementioned methods, a third approach was carried out. This was inspired by a work published by Li et al., in which a solvothermal synthesis was used to grow nanostructured $\mathrm{V}_{2} \mathrm{O}_{5}$ on a CNF substrate; such material was then used as electrode for an asymmetrical electrochemical supercapacitor. The authors described the use of relatively straightforward solvothermal and annealing processes that were followed here. First, CNFs were prepared using the previously described (Section 5.2.1) method and then a solution of VOTIP in isopropanol was prepared and put inside an autoclave with a sheet of CNFs. The solvothermal process was then performed by heating the sealed autoclave to 200 ${ }^{\circ} \mathrm{C}$ for 10 hours. After recovering and washing the material with de-ionised water and ethanol, SEM images were acquired to evaluate its nanomorphology after the solvothermal step (Figure 6.7). The images show the presence of nanosheets that are only a few tenths of manometers thick and protruding from the fibres. Such 


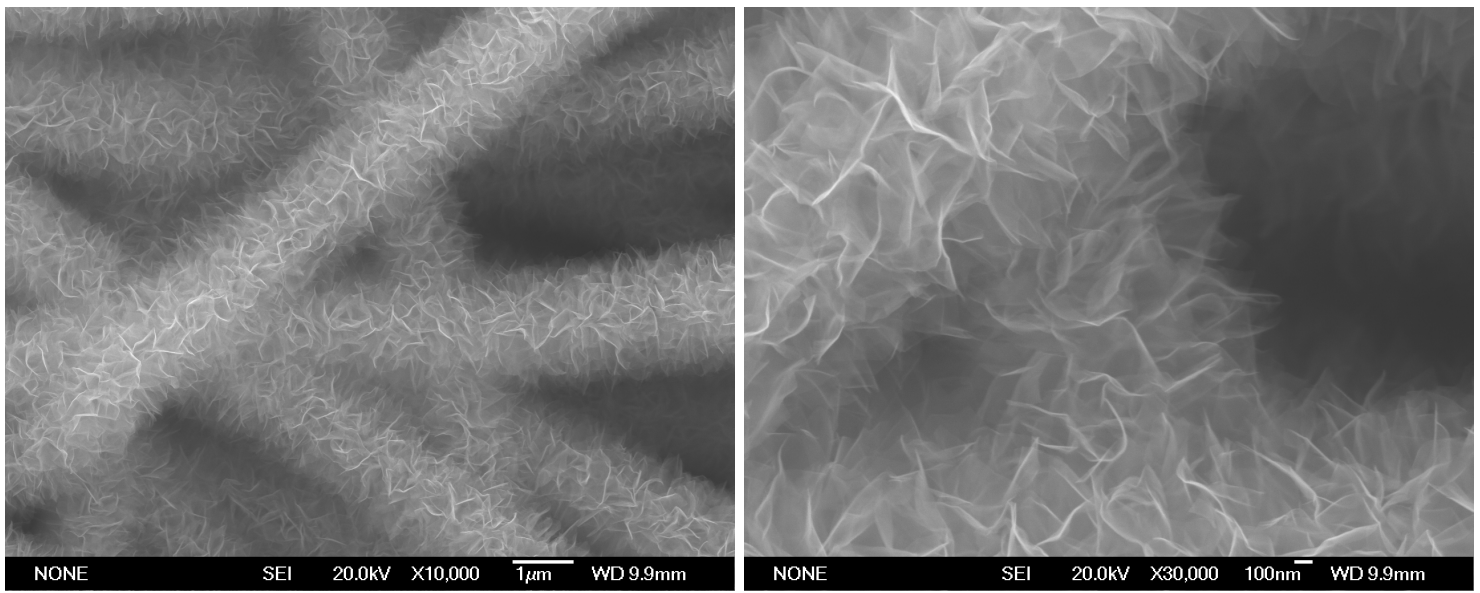

FIGURE 6.7: SEM micrographs of core-sheath nanofibres (prior to the annealing step) which were prepared by solvothermal growth, according to the procedure described by Li et al. ${ }^{5}$

morphology is almost identical to what reported by the authors, indicating that the process was successfully replicated.

Following the solvothermal synthesis, the authors claimed to have used an annealing step at $320{ }^{\circ} \mathrm{C}$ in air, after which the precursor gets converted to polycrystalline $\mathrm{V}_{2} \mathrm{O}_{5}$ while leaving the carbon core unaltered. After replicating the annealing step, however, the material appeared much less flexible than what was claimed by the authors, possibly indicating the destruction of the carbon core. This hypothesis was verified by performing a TGA analysis in air of the sample (Figure 6.8). The thermogram shows that little to no mass is lost in the temperature range between 120 and $650{ }^{\circ} \mathrm{C}$, indicating that most of the carbon content of the material had already been lost in the annealing process. This result was unexpected, as the the preservation of the carbon core was also confirmed by TGA by the authors. ${ }^{5}$ STEM-EDX maps (Figure 6.9) further confirmed this result, showing the absence of a carbon core in the fibres. The nanomorphology of the sheath layer, on the other hand, was not significantly affected by the heat treatment. The only notable difference is the presence of small holes and defects in the nanosheets, possibly caused by the formation of $\mathrm{V}_{2} \mathrm{O}_{5}$ nanocrystals (Figure A.16).

In order to better understand the process, the crystal properties of the material prior to the annealing step were studied by XRD. It can be seen from the data plot in Figure 6.10 that the as-prepared core-sheath NFs already show certain degrees of crystallinity, as demonstrated by the presence of a few weak diffraction 


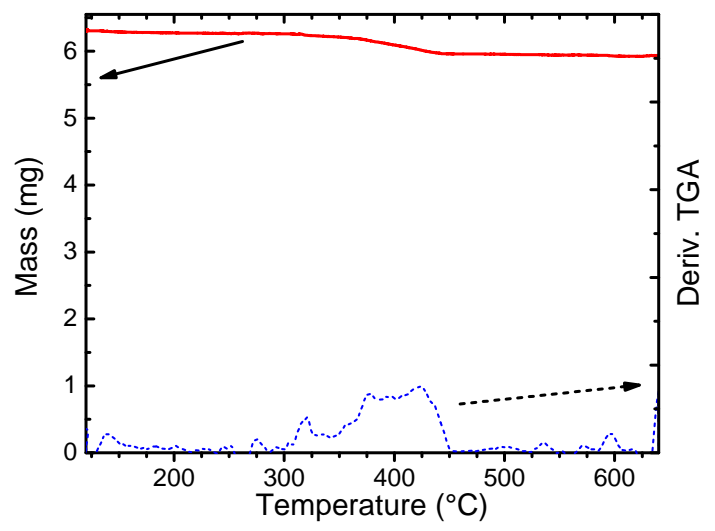

FIGURE 6.8: Thermogravimetric analysis of core-sheath nanofibres, prepared by solvothermal growth, annealed at $320^{\circ} \mathrm{C}$ in air.

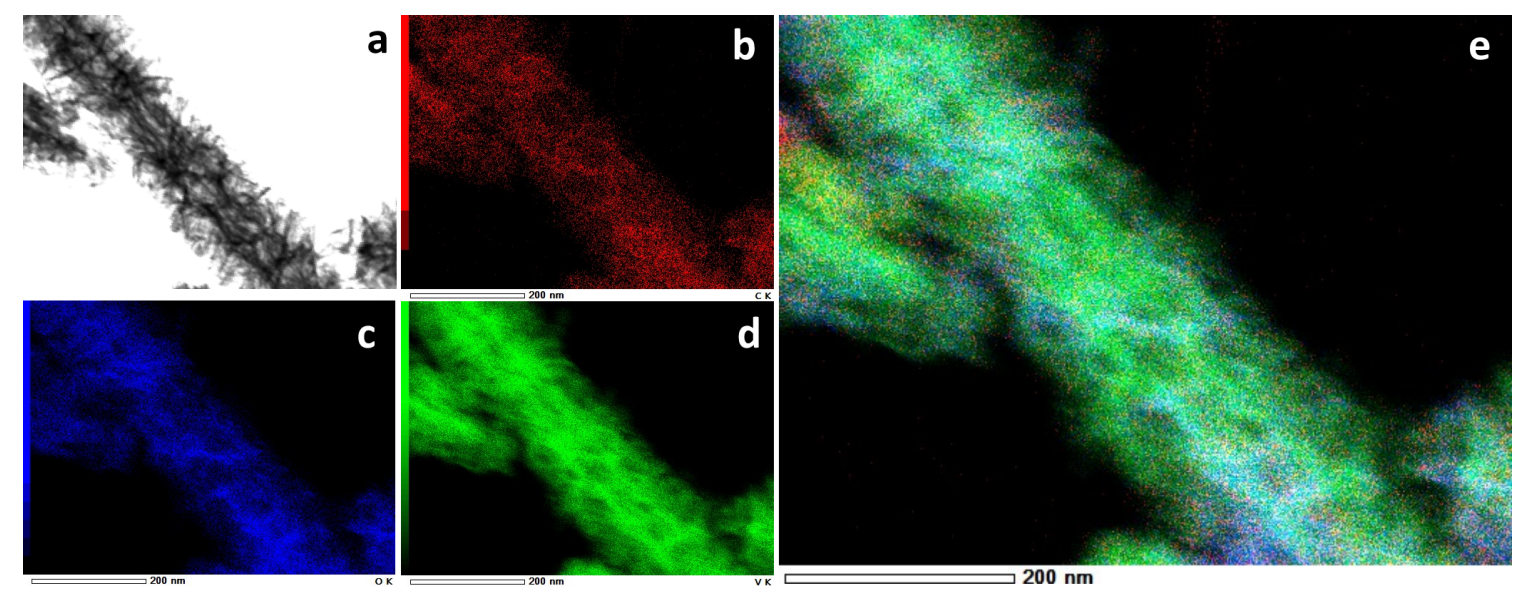

FIGURE 6.9: STEM-EDX elemental maps of core-sheath nanofibres, prepared by solvothermal growth, annealed at $320^{\circ} \mathrm{C}$ in air: STEM-BF image (a), carbon (b), oxygen (c), vanadium (d), and overlay (e) maps.

peaks. Although a suitable database match could not be found for the crystal phase present in the compound, other publications attribute some of the diffraction peaks observed to the hydrated phase of vanadium $(\mathrm{V})$ oxide, $\mathrm{V}_{2} \mathrm{O}_{5} \cdot \mathrm{xH}_{2} \mathrm{O}$. $^{6-9}$ The information gathered from XRD data hinted at the possibility of annealing the material using different conditions: it was hypothesised that a milder, longer annealing period could work in converting the sheath material while leaving the core material intact. Furthermore, using a vacuum heating could perhaps help in removing the water content of the material Therefore, the nanofibres were placed in a vacuum oven and heated at $240{ }^{\circ} \mathrm{C}$. This proved to be a successful strategy, 


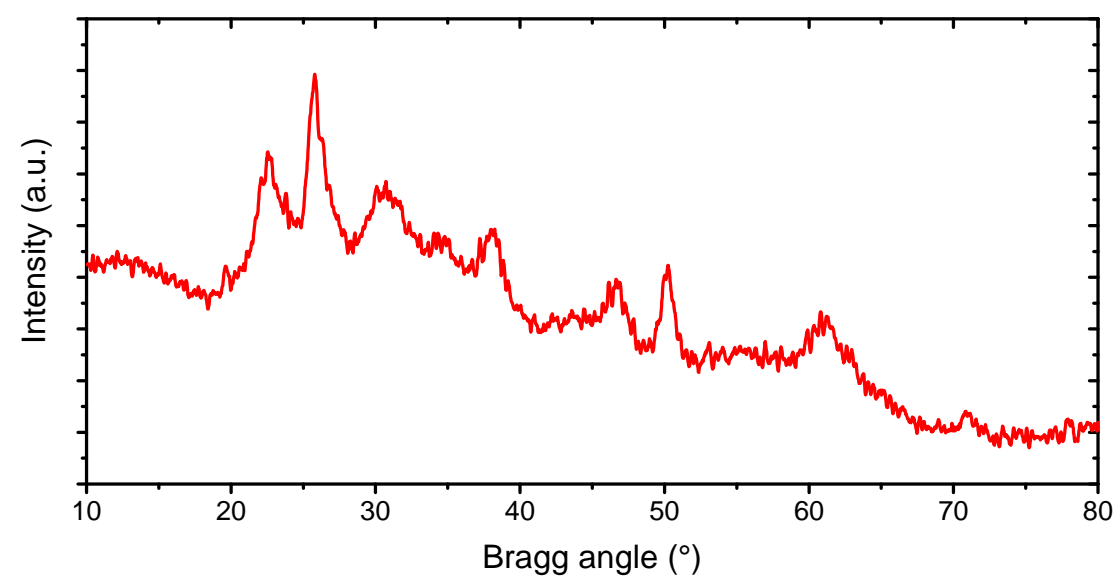

FIGURE 6.10: X-ray diffractogram of core-sheath nanofibres, prepared by solvothermal growth, prior to the annealing step.

as the sheath material was effectively converted to the desired crystal phase. As demonstrated from the XRD data in Figure 6.11, a series of peaks in good agreement with one of the ICDD database patterns for orthorombic $\mathrm{V}_{2} \mathrm{O}_{5}$ can be observed in the diffractogram. In addition, high resolution TEM imaging was performed on the material, showing the polycrystalline character of the nanosheets and the presence of circular cavities roughly 2-3 nm in diameter (Figure 6.12). Finally, STEM-EDX elemental mapping was performed on the sample, confirming the presence of a carbon core. Based on this data, this process was deemed as

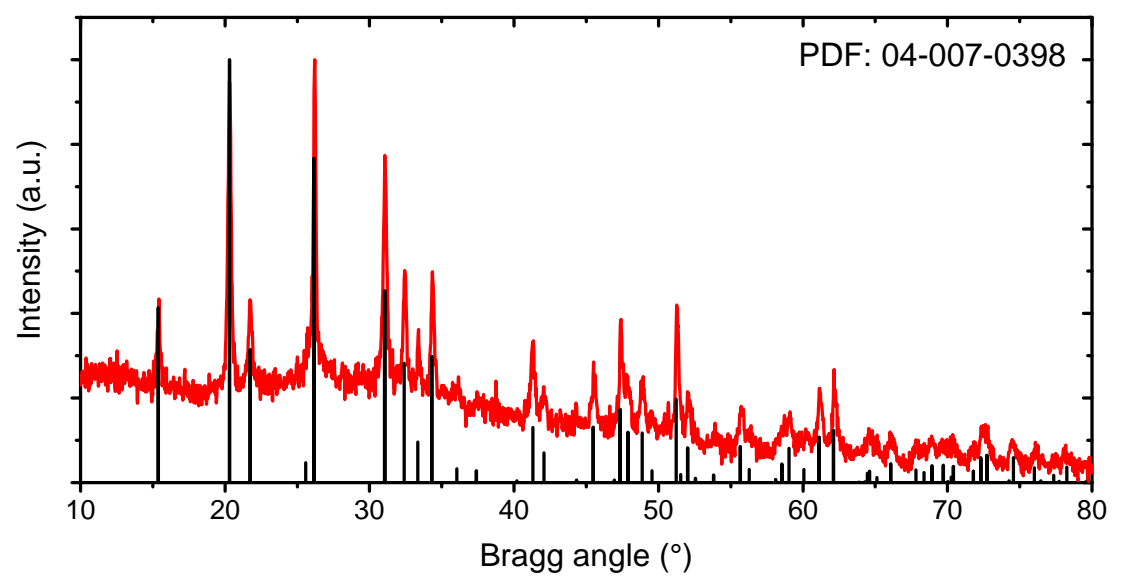

FIGURE 6.11: X-ray diffractogram of core-sheath $\mathrm{C} / \mathrm{V}_{2} \mathrm{O}_{5}$ nanofibres, prepared by solvothermal growth, annealed at $240{ }^{\circ} \mathrm{C}$ under vacuum. 
suitable to obtain the desired material nanomorphology (Figure 6.13). Therefore, it was decided to move on to the next step in the project and test the material as a cathode for AIBs.

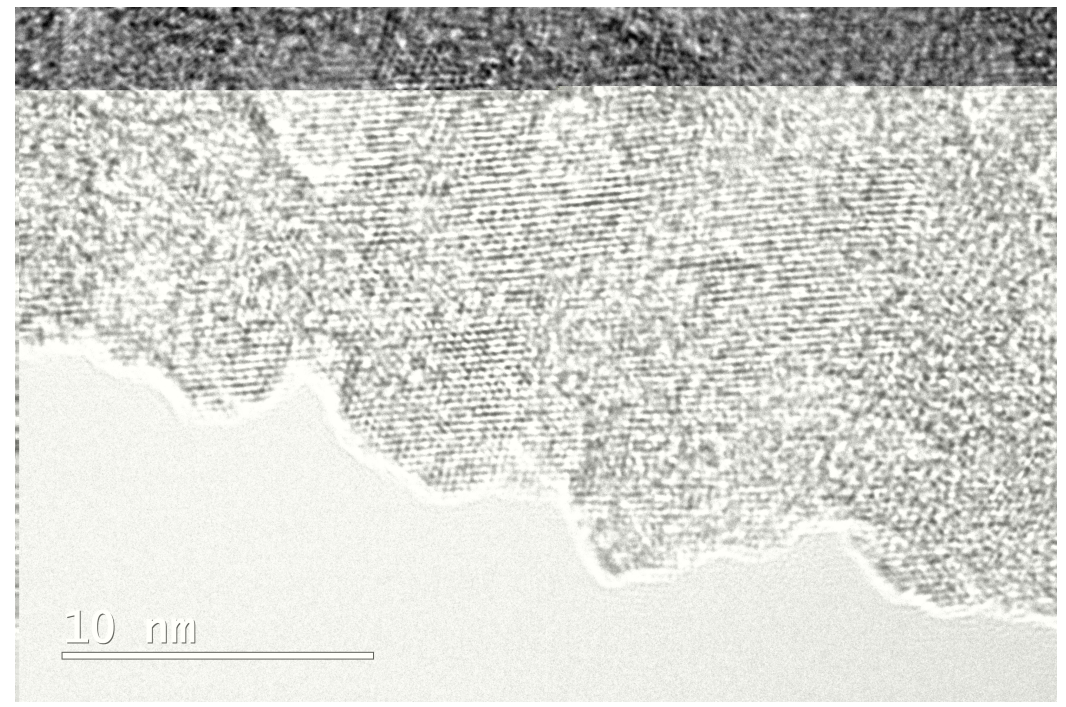

FIGURE 6.12: HRTEM micrograph of core-sheath $\mathrm{C} / \mathrm{V}_{2} \mathrm{O}_{5}$ nanofibres, prepared by solvothermal growth, annealed at $240{ }^{\circ} \mathrm{C}$ under vacuum.
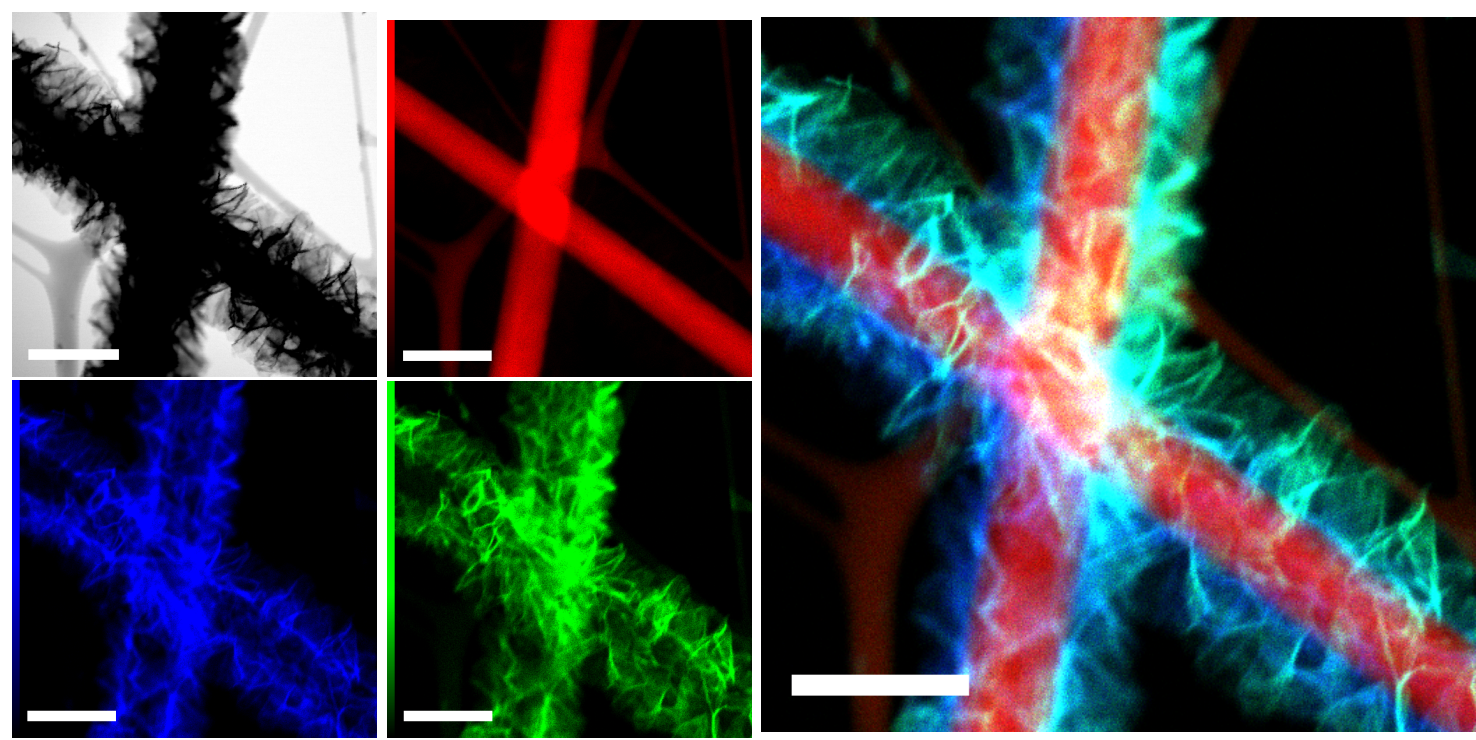

FIGURE 6.13: STEM-EDX elemental maps of core-sheath $\mathrm{C} / \mathrm{V}_{2} \mathrm{O}_{5}$ nanofibres, prepared by solvothermal growth, annealed at $240{ }^{\circ} \mathrm{C}$ under vacuum: STEM-BF image (top left), carbon (red), oxygen (blue), vanadium (green) maps, and overlay image (right). Scale bar: $2 \mu \mathrm{m}$. 


\subsection{Electrochemical tests}

Swagelok-type cells were assembled using the core-sheath NF as the cathode. Thanks to the preservation of the carbon core, the material still retained the good mechanical properties of CNFs and could be directly cut into appropriately shaped discs and used without further treatments. A galvanostatic chargedischarge test was initially performed on the device using a current rate of $100 \mathrm{~mA}$ $\mathrm{g}^{-1}$. Assuming that $\mathrm{V}_{2} \mathrm{O}_{5}$ would predominantly be the active material, the test implemented a cycling protocol between 0.02 and $2.45 \mathrm{~V}$, starting with a discharge sequence.
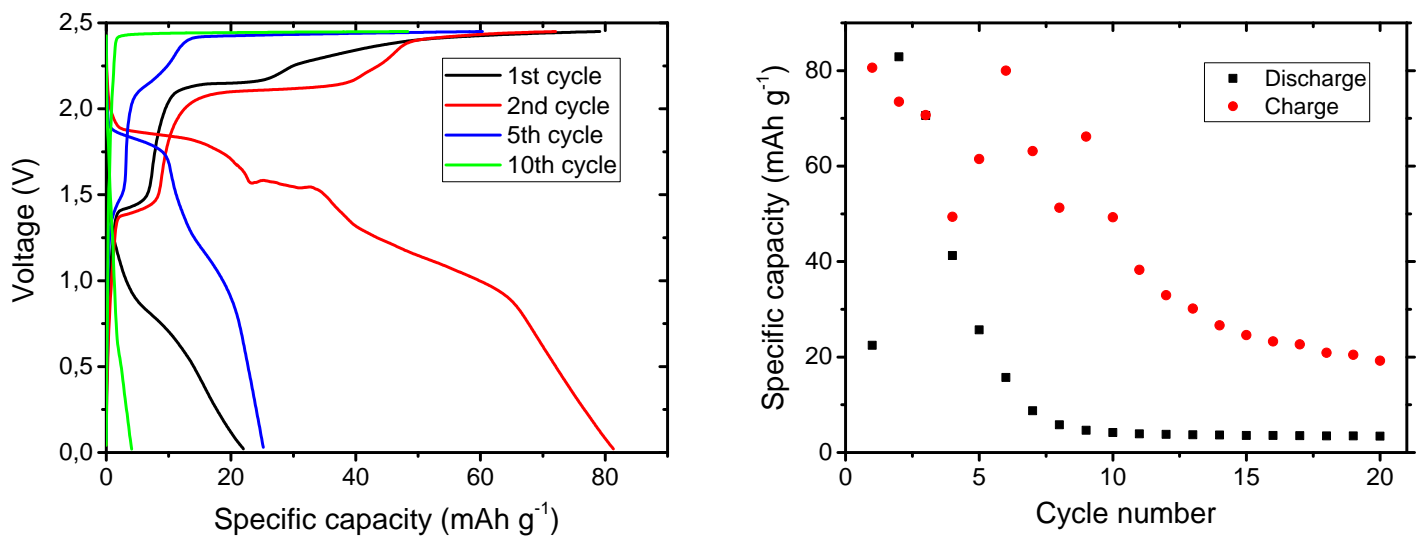

FIGURE 6.14: (Left) Galvanostatic charge-discharge cycle profiles of a Swageloktype cell built using core-sheath $\mathrm{C} / \mathrm{V}_{2} \mathrm{O}_{5}$ nanofibres as cathode. The voltage reported in this plot is the cell potential. (Right) Specific capacities obtained in a typical galvanostatic charge-discharge test of a Swagelok-type cell built using core-sheath $\mathrm{C} / \mathrm{V}_{2} \mathrm{O}_{5}$ nanofibres as cathode.

An interesting behaviour was observed in this experiment: it can be seen from Figure 6.14 (Left) that the first discharge cycle features only a weakly defined plateau at about $0.8 \mathrm{~V}$ and yields an overall modest capacity of about $21 \mathrm{mAh} \mathrm{g}^{-1}$. The following charging sequence, however, reveals a series of sharp features: a short plateau at about $1.4 \mathrm{~V}$ and two longer ones around $2.1 \mathrm{~V}$ and above. The specific capacity relative to the first charge is also considerably longer, at about 80 $\mathrm{mAh} \mathrm{g}^{-1}$. The following discharge sequence then shows yet more new plateaus that weren't present in the first cycle, at 1.8, 1.6, and 1.2 V. The specific capacity yielded is also notably higher, at roughly $81 \mathrm{mAh} \mathrm{g}^{-1}$. This behaviour was completely unexpected, as it strongly differs from what was observed for pure 
$\mathrm{V}_{2} \mathrm{O}_{5}$ nanofibres (Section 4.3.2). In the following cycles, the features in the chargedischarge profiles become more regular, however, the capacity fades quite rapidly and stabilises around $4 \mathrm{mAh} \mathrm{g}^{-1}$ after about 10-11 cycles.

\subsubsection{The hypothesis of a mixed-ion intercalation mechanism}

The behaviour observed in the aforementioned galvanostatic charge-discharge test is quite complex and hard to interpret, but a few considerations can be made. The presence of a much longer charge sequence than the corresponding discharge in the first cycle, accompanied by the appearance of new features, hints at the possibility of multiple processes taking place in the material. Such processes appear to be happening within different voltage ranges: one process below $1.5 \mathrm{~V}$ and the other between 1.5 and $2.45 \mathrm{~V}$. One preliminary interpretation is that the material could undergo a mixed-ion intercalation mechanism. In this case, when the battery operates at lower voltages (roughly below $1.5 \mathrm{~V}$ ), the primary mechanism during the discharge would be the expected insertion of $\mathrm{Al}^{3+}$ ions into $\mathrm{V}_{2} \mathrm{O}_{5}$, as demonstrated by previous reports. ${ }^{10}$ At higher voltages, however, the galvanostatic profile somewhat resembles the one observed for graphitic materials (see also Figure A.8). This would suggest that at higher voltages, a similar process would take place: the intercalation of $\mathrm{AlCl}_{4}^{-}$ions during the charging phase and their deintercalation during the discharge phase. It is unclear at this stage whether $\mathrm{AlCl}_{4}^{-}$intercalation is in fact taking place in the material or whether it could take place in the carbon core or in the $\mathrm{V}_{2} \mathrm{O}_{5}$ sheath. The reason for this uncertainty is based on the results outlined in the previous chapter, were the characteristics of the carbon core appeared to not allow the insertion of $\mathrm{AlCl}_{4}^{-}$ions. Therefore, if such process is in fact taking place, one of two possible consequences would be implied:

1. The intercalation is taking place in the $\mathrm{V}_{2} \mathrm{O}_{5}$ sheath component. This assumption would be in disagreement with previous literature arguing that the intercalation mechanism in $\mathrm{V}_{2} \mathrm{O}_{5}$ only involves $\mathrm{Al}^{3+}$ ions.

2. The intercalation process takes place in the carbon core of the fibres. Therefore, the previous assumptions regarding the ability of CNFs to allow intercalation of $\mathrm{AlCl}_{4}^{-}$(see also Section 5.4) would either be incorrect or at least 
partially incomplete, and the presence of the sheath material could somehow promote the insertion of the chloroaluminate ions in the carbon core.

One observation in favour of this second hypothesis is the fact that during the first cycles, the asymptotic voltage plateau behaviour correlated with the formation of an SEI (as discussed in Section 5.2.2), is not observed. This could indicate that the presence of a $\mathrm{V}_{2} \mathrm{O}_{5}$ sheath somehow inhibits this unwanted process, and therefore allows the intercalation process to take place instead. This is also coherent with the hypothesis that surface defects are responsible for the early onset of electrolyte degradation and formation of SEI. The presence of a $\mathrm{V}_{2} \mathrm{O}_{5}$ sheath could effectively shield such defects, removing them from direct contact with the electrolyte. This is also supported by the fact that after the first few cycles, the discharge capacity decreases to abysmal values and an asymptotic voltage plateau appears: such change in electrochemical behaviour could be caused by the disruption of the $\mathrm{V}_{2} \mathrm{O}_{5}$ sheath and the termination of the shielding effect. Consequently, this would revert the energy storage mechanism to the one observed in pure, non-coated CNFs.

In order to further test the hypothesis of a multiple-ion intercalation mechanism, a second galvanostatic charge-discharge test, using the same voltage range and cycling rate, was performed. This time, however, a protocol starting with a charging step was used: this way, if the $\mathrm{AlCl}_{4}^{-}$intercalation were to take place, then a charging plateau should be detectable in the first charging step, even without performing any previous discharging steps. It can be seen from Figure 6.15 that two charging plateaus indeed take place in the first cycle, which are therefore likely associated with the insertion of $\mathrm{AlCl}_{4}^{-}$. Furthermore, the first discharge sequence yields a discharge capacity of $71 \mathrm{mAh} \mathrm{g}^{-1}$, a value much higher than the one obtained in the test starting with the discharge step (Figure 6.14). This result indicates that a certain degree of charging has taken place in the first step, which adds up to the overall discharge capacity observed in the first cycle.

This test then suggests that when core-sheath $\mathrm{C} / \mathrm{V}_{2} \mathrm{O}_{5}$ nanofibres are used as a cathode in AIBs, the freshly assembled battery device would start in an intermediate state of charge. Therefore either a charging or a discharging step could be first performed in the cycling protocol. Although this test does not provide concrete proof, it indicates that a mixed-ion mechanism is definitely possible for this material: 


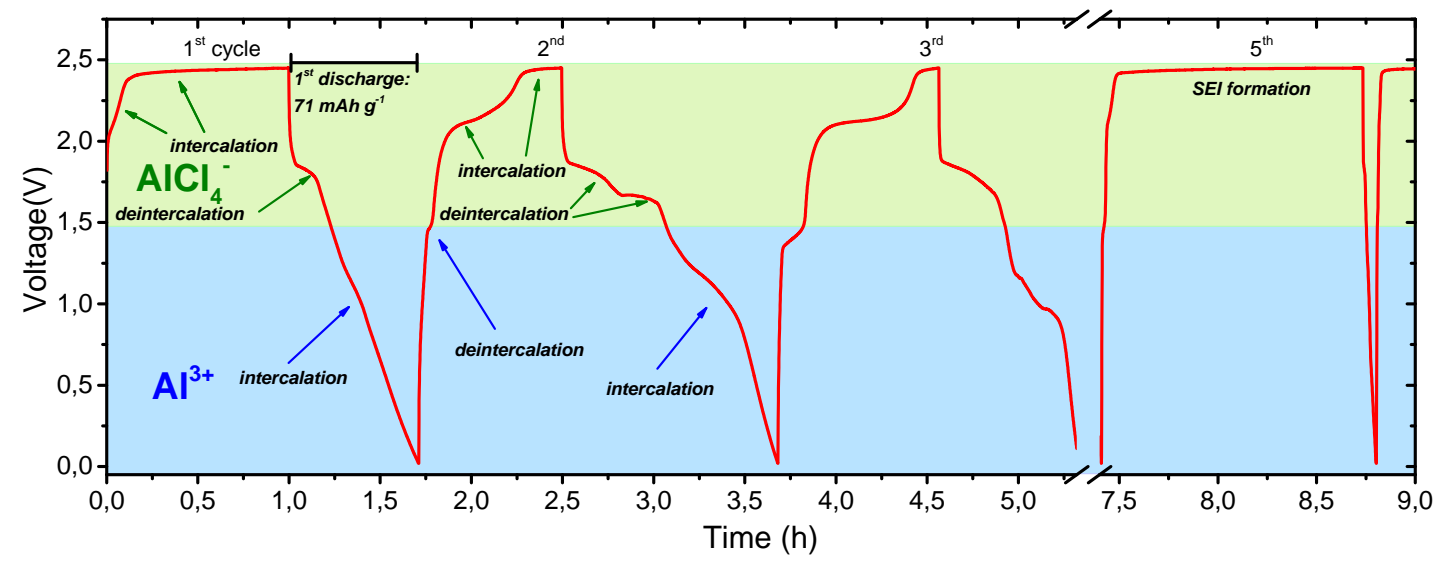

FIGURE 6.15: Galvanostatic cycling (at a cycling rate of $100 \mathrm{~mA} \mathrm{~g}^{-1}$ ), starting with a charge sequence, of a Swagelok-type cell built using core-sheath $C / \mathrm{V}_{2} \mathrm{O}_{5}$ nanofibres as cathode. The voltage reported in this plot is the cell potential.

- If the first step performed on the device is a charging phase, then the first event taking place at the cathode is the intercalation of $\mathrm{AlCl}_{4}^{-}$. In the following discharge step, the deintercalation of $\mathrm{AlCl}_{4}^{-}$then takes place from 2.45 to $1.5 \mathrm{~V}$ and the insertion of $\mathrm{Al}^{3+}$ takes place as the potential decreases below $1.5 \mathrm{~V}$. Finally, $\mathrm{Al}^{3+}$ is deintercalated in the following charge cycle, completing the cycle. This scenario is shown in Figure 6.15.

- If instead the first step performed on the device is a discharging phase, then the first event taking place at the cathode is the intercalation of $\mathrm{Al}^{3+}$. The ions are then then deintercalated in the following discharge step until the $1.5 \mathrm{~V}$ potential is reached, after which the intercalation of $\mathrm{AlCl}_{4}^{-}$takes place. In the next discharge step, $\mathrm{AlCl}_{4}^{-}$are deintercalated from 2.45 to $1.5 \mathrm{~V}$, completing the cycle.

Further evidence for this hypothesis is given by the high discharge capacity values obtained in the first few cycles, which are much higher than the ones obtained both for CNFs and $\mathrm{V}_{2} \mathrm{O}_{5}$ NFs in the previous chapter. A maximum discharge capacity of about $118 \mathrm{mAh} \mathrm{g}^{-1}$, corresponding to a remarkable specific energy of about $156 \mathrm{mWh} \mathrm{g}^{-1}$, was obtained in the second cycle (Figure A.17). Such high values suggest that both the core and the sheath components of the cathode material are likely contributing to the energy storage mechanism. Assuming that both components could individually undergo their respective mechanisms, this 
would imply that both processes could be happening at the same time in a coresheath NF: intercalation of $\mathrm{AlCl}_{4}^{-}$ions into carbon during the charging phase and insertion of $\mathrm{Al}^{3+}$ into $\mathrm{V}_{2} \mathrm{O}_{5}$ during the discharging phase. This is a very interesting feature, as the intercalation of multiple different ionic species correlates to a more efficient utilisation of the chloroaluminate electrolyte. ${ }^{11,12}$

In both the experiments the performance is unfortunately quite unstable, as discharge capacities fade rather quickly. A possible explanation for this could be that the the mechanical stress induced by the intercalation and deintercalation reactions causes the gradual detaching of the $\mathrm{V}_{2} \mathrm{O}_{5}$ sheath. This would cause a loss in discharge capacity and disrupt the synergistic interaction between the core and sheath materials that allowed the multiple-ion mechanism to take place. This is also hinted by the change in shape of the galvanostatic profiles: after the first two or three cycles, the features and plateaus of the charging and discharging sequences start becoming gradually less pronounced, suggesting the disintegration of the cathode. This hypothesis also fits with the previous assumption regarding the possible role of the $\mathrm{V}_{2} \mathrm{O}_{5}$ sheath in inhibiting SEI formation; the removal of the $\mathrm{V}_{2} \mathrm{O}_{5}$ sheath would mean that the surface of CNFs is again exposed to the electrolyte, causing the same type of behaviour explained in the previous chapter (see also Section 5.4). It can be seen that the tenth and fifth cycles in Figures 6.14 and 6.15 , respectively, closely resemble the galvanostatic profiles of a device built using pristine CNFs. A very long plateau is observed near the upper voltage cutoff that once again suggests the formation of an SEI, and is paired with an extremely short discharging sequence without any outstanding features.

Further evidence for the disintegration of the $\mathrm{V}_{2} \mathrm{O}_{5}$ sheath can be seen in the comparison of cyclic voltammograms performed on the device before and after the charging process, in Figure 6.16. The CV performed on the pristine material shows an overall high current intensity, and a series of peaks which loosely match the position of the voltage plateaus in the charge-discharge data, and is therefore imputable to the intercalation of $\mathrm{Al}^{3+}$ and $\mathrm{AlCl}_{4}^{-}$ions. After galvanostatic cycling, however, the current density decreases notably, and the peaks are much less intense relatively to the hysteretic baseline. The sharp increase in current beyond $2.25 \mathrm{~V}$ is also now present, suggesting that the carbon core is now likely exposed and promoting the formation of the SEI. 

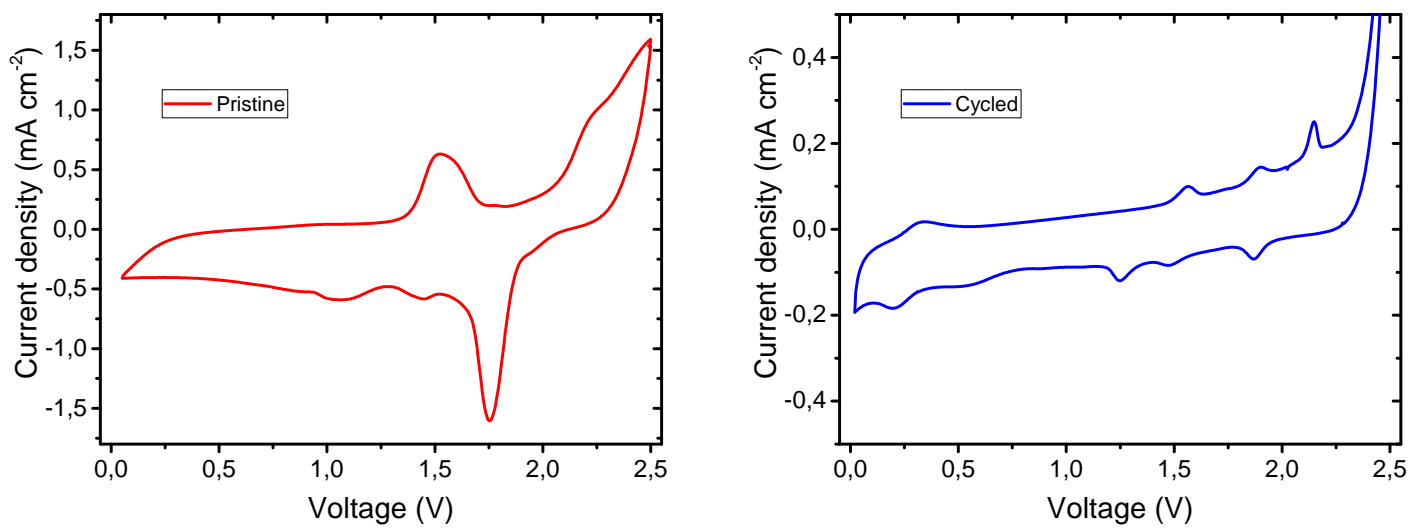

FIGURE 6.16: Cycling voltammograms of a device built using core-sheath $\mathrm{C} / \mathrm{V}_{2} \mathrm{O}_{5}$ nanofibres, before (Left) and after (Right) performing 20 chargedischarge cycles at $100 \mathrm{~mA} \mathrm{~g}^{-1}$. The current densities are referred to the projected cathode area; the voltage reported in these plots is the cell potential.

\subsection{Ex situ studies}

In order to investigate the energy storage process and the possible presence of a mixed-ion mechanism in the material, an ex situ TEM analysis was performed. First, a device using the core-sheath $\mathrm{C} / \mathrm{V}_{2} \mathrm{O}_{5} \mathrm{NFs}$ as cathode was assembled. Then, a galvanostatic charging protocol consisting of a charge, a discharge, and a charge (i.e. one and a half cycles) at a current rate of $100 \mathrm{~mA} \mathrm{~g}^{-1}$ was performed on the battery. Finally, the cell was disassembled and the cathode was observed using TEM and STEM-EDX mapping, to assess the aluminium and chlorine content in the fibres. The presence and spatial location of chlorine in the fibres would be especially revealing, as it could indicate that the $\mathrm{AlCl}_{4}^{-}$ion is involved in the energy storage mechanism, and whether it interacts preferentially with the core or the sheath layer. There are a few reasons for choosing this specific chargingdischarging protocol for the experiment: first of all, imaging the cathode after a limited number of cycles is preferable, due to the probability for the sheath layer to disintegrate. Furthermore, previous tests (Figures 6.14 and 6.15) have shown that the largest specific capacities are obtained in the second cycle of the device. Therefore, it was hypothesised that by disassembling the device in the second cycle, the largest perturbation of the system would be observed, which would ideally correspond the most evident change in elemental composition of the material. Finally, the main purpose of the experiment is to verify the hypothesis that both $\mathrm{Al}^{3+}$ and 

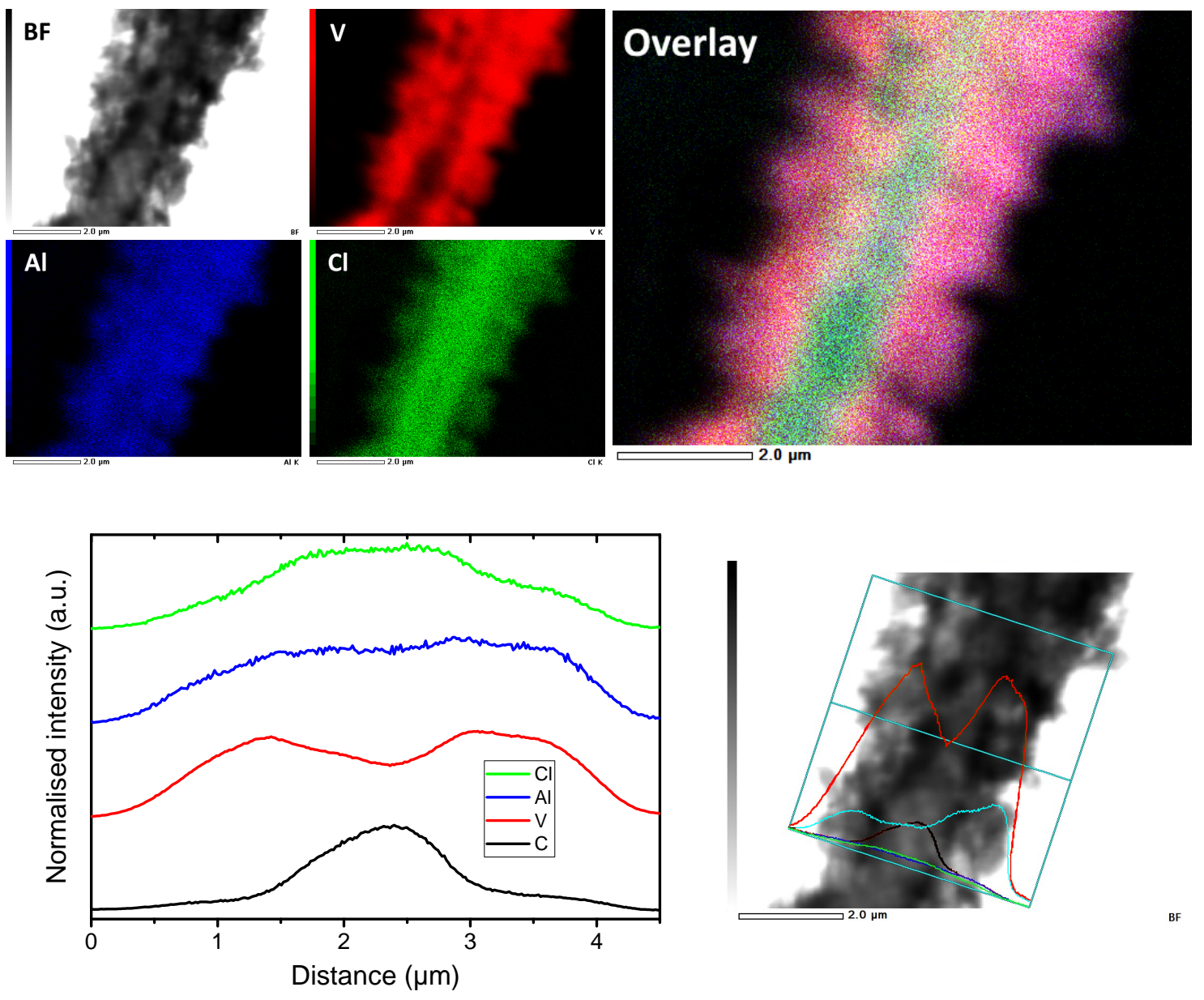

FIGURE 6.17: Ex situ STEM-EDX data of charged-state core-sheath $\mathrm{C} / \mathrm{V}_{2} \mathrm{O}_{5} \mathrm{NF}$. (Top level) Elemental maps: STEM-BF image (top left), vanadium (red), aluminium (blue), chlorine (green) maps, and overlay image (right). (Bottom level) Linear intensity profiles of individual elements across a single fibre (left), and corresponding integration area (right).

$\mathrm{AlCl}_{4}^{-}$are involved in the mechanism. On one hand, the intercalation of $\mathrm{Al}^{3+}$ into $\mathrm{V}_{2} \mathrm{O}_{5}$ has been confirmed by previous reports ${ }^{10}$ to happen during the discharge process. On the other hand, the intercalation of $\mathrm{AlCl}_{4}^{-}$in this material was only hypothesised by its galvanostatic charge-discharge behaviour. Such experiments suggested that if the intercalation $\mathrm{AlCl}_{4}^{-}$were happening at the cathode, it would have to take place during the charging step. Therefore, by disassembling the device and observing the cathode in its charged state, detecting the presence of both $\mathrm{Al}$ and $\mathrm{Cl}$ by STEM-EDX mapping would be a possible confirmation that such process indeed takes place. 
It can be observed from Figure 6.17 that both aluminium and chlorine are present in the nanofibres after the charge-discharge protocol applied. This is a remarkably different result to the one previously reported by Gu et al., ${ }^{10}$ who claimed that only $\mathrm{Al}$ was present in their material. This indicates that core-sheath $\mathrm{C} / \mathrm{V}_{2} \mathrm{O}_{5}$ likely undergoes an energy storage mechanism involving the $\mathrm{AlCl}_{4}^{-}$ion. However, it can be seen that the intensity of the $\mathrm{Cl} \mathrm{K}$ peak is notably more pronounced in the core section of the fibre, where $\mathrm{C}$ is present. On the other hand, its intensity is visibly lower in the $\mathrm{V}_{2} \mathrm{O}_{5}$ sheath. This can be seen as evidence that the $\mathrm{AlCl}_{4}^{-}$ion can effectively intercalate in the carbon core in a similar fashion to graphitic cathode materials, but not into the $\mathrm{V}_{2} \mathrm{O}_{5}$ sheath, which is coherent with the previous reports. ${ }^{10}$

It is also worth noting that the intensity of the $\mathrm{Al}$ peak, on the other hand, is mostly uniform across the entire fibre. Although this result may at first appear contradictory, it is in fact consistent with the previous observations and assumptions. Unsurprisingly, the presence of $\mathrm{Al}$ in the carbon core is justified by the intercalation of the $\mathrm{AlCl}_{4}^{-}$ion during the charging step. On the other hand, the assumed process taking place in the $\mathrm{V}_{2} \mathrm{O}_{5}$ sheath is the insertion of $\mathrm{Al}^{3+}$ ions during the discharge phase. Therefore, the intensity of $\mathrm{Al}$ should be lower than the one observed in the sample, which is in a charged state. However, the high concentration of $\mathrm{Al}$ in the sheath layer can be justified by the entrapment of of $\mathrm{Al}^{3+}$ ions in the $\mathrm{V}_{2} \mathrm{O}_{5}$ layer due to to the poor reversibility of the intercalation-deintercalation process. This is consistent with the results observed for $\mathrm{V}_{2} \mathrm{O}_{5}$ cathodes in Chapter 4 and by earlier literature reports. ${ }^{10,13,14}$

A depth profile study using X-ray photoelectron spectroscopy was also performed on the same cathode from the ex situ TEM study. The $\mathrm{Ar}^{+}$ion beam present in the XPS instrument was used to etch the surface of the sample and remove portions of the material; the approximate etching rate for this instrument based on previous tests on a $\mathrm{Ta}_{2} \mathrm{O}_{5}$ substrate was about $1 \mathrm{~nm}$ per minute. A total of fifteen 10-minute sputtering cycles were run, and a survey spectrum in the binding energy range of 0-600 eV was collected after each cycle. The elemental concentrations for carbon, vanadium, and chlorine were then estimated individually for each of the spectra acquired by integrating the area of their characteristic peaks: 1s for carbon, $2 p$ for vanadium, and $2 p$ for chlorine. Unfortunately, due to a combination of the low sensitivity of the XPS technique for aluminium ${ }^{15}$ and the overlap between its 
most intense (2p) peak and the 3s peak of vanadium, a reasonably accurate depth profile for such element could not be obtained.

While the previous STEM-EDX elemental mapping analysis which focused on a single fibre, the use of this technique should provide a more averaged, "bulk"type information of the elemental composition of the sample. This is because the instrument scans across a larger area compared to that used in the previous TEM experiment, therefore sampling a larger quantity of material. It is also worth noting that although the thickness of the "sheath" layer is several hundreds of $\mathrm{nm}$ and the ion beam would theoretically only remove about $150 \mathrm{~nm}$ of material at best (according to the $\mathrm{Ta}_{2} \mathrm{O}_{5}$ substrate test), such layer of the material is composed of very thin $\mathrm{V}_{2} \mathrm{O}_{5}$ sheets, and a large amount of it is composed of empty space. Furthermore, based on the diameter of the nanofibre observed in the previous TEM study, it would appear that part of the $\mathrm{V}_{2} \mathrm{O}_{5}$ has already been lost as a consequence of the galvanostatic process. Therefore, the validity of this technique in identifying the elemental composition of the "core" layer of the nanofibres was deemed reasonable and worth attempting.

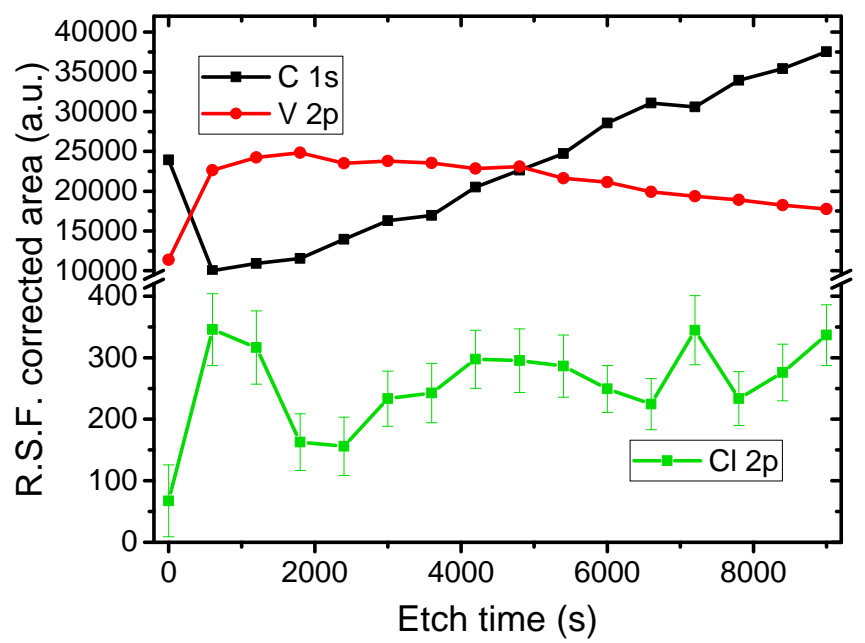

FIGURE 6.18: Ex situ XPS depth profile of charged-state core-sheath $\mathrm{C} / \mathrm{V}_{2} \mathrm{O}_{5} \mathrm{NF}$. The error bars on the $\mathrm{Cl} 2 \mathrm{p}$ data points refer to the standard deviation calculated on the integration area by the CasaXPS software.

The depth profile data is reported in Figure 6.18. It can be seen from the plot that the integrated areas for the C $1 \mathrm{~s}$ and V $2 \mathrm{p}$ peaks follow an ascending and descending trending, respectively. This is expected given the morphology of the sample; furthermore, by correcting the values according to their relative strength 
factor, the intensity of the carbon peak actually surpasses that of the vanadium peak after an etching time of approximately 4800 seconds, suggesting that the core level of the nanofibres could have possibly been reached by the prolonged etching cycles. This assumption is however mostly speculative, as the data obtained from the XPS spectra reflects the composition of an ensemble of nanofibres illuminated by the X-ray source. Furthermore, other factors such as the integration range of the peaks in the spectra could also be influencing the intensities of the peaks, leading to an overestimation or underestimation of the elemental composition. Possibly the most important information that can be observed from the depth profile is the trend of the $\mathrm{Cl} 2 \mathrm{p}$ peak: similarly to carbon, that the area of the $\mathrm{Cl}$ $2 \mathrm{p}$ peak also follows a roughly increasing trend. Although the data points feature quite a significant deviation, this can be seen as further indication that chlorine is present in the carbon core of the fibres, as a consequence of the intercalation of $\mathrm{AlCl}_{4}^{-}$ions during the charging step. This result therefore further supports the hypothesis of a multi-ion intercalation mechanism taking place in the material. It is worth noting that the first three data points relative to the $\mathrm{Cl} 2 \mathrm{p}$ depth profile follow a seemingly contrasting trend compared to the remaining data. Although many factors could be causing these outliers, one possible explanation for this is that a certain amount of electrolyte or SEI layer could still be present on the nanofibres, resulting in a high concentration of chlorine-containing species in the outermost layers of the sample. This is also partially supported by the presence of a trend inversion in the carbon and vanadium concentration for the first data point as well, which could be attributed to the presence of [EMIm] ${ }^{+}$ions or other organic species deriving from the decomposition of the electrolyte. It is unclear, however, how this trend inversion only applies for the first data point in the $\mathrm{C}$ and $\mathrm{V}$ integration areas, and instead applies for the second and third for the $\mathrm{Cl}$ peak. One possible reason could be the presence of adventitious carbon in the outermost layer of the sample, accounting for a boost in the intensity of the carbon peak, but not for that of the chlorine peak.

Finally, ex situ SEM was performed on a cathode after running 20 cycles at 100 $\mathrm{mA} \mathrm{g}^{-1}$ to verify the hypothesis that the cycling process causes the disintegration of the $\mathrm{V}_{2} \mathrm{O}_{5}$ in the material. It can be seen from Figure 6.19 that the vanadium oxide sheath has been completely removed from the fibres, and they can be seen 
to be coated in an SEI film. This finding confirms that the $\mathrm{V}_{2} \mathrm{O}_{5}$ sheath gets disintegrated in the charging-discharging process.

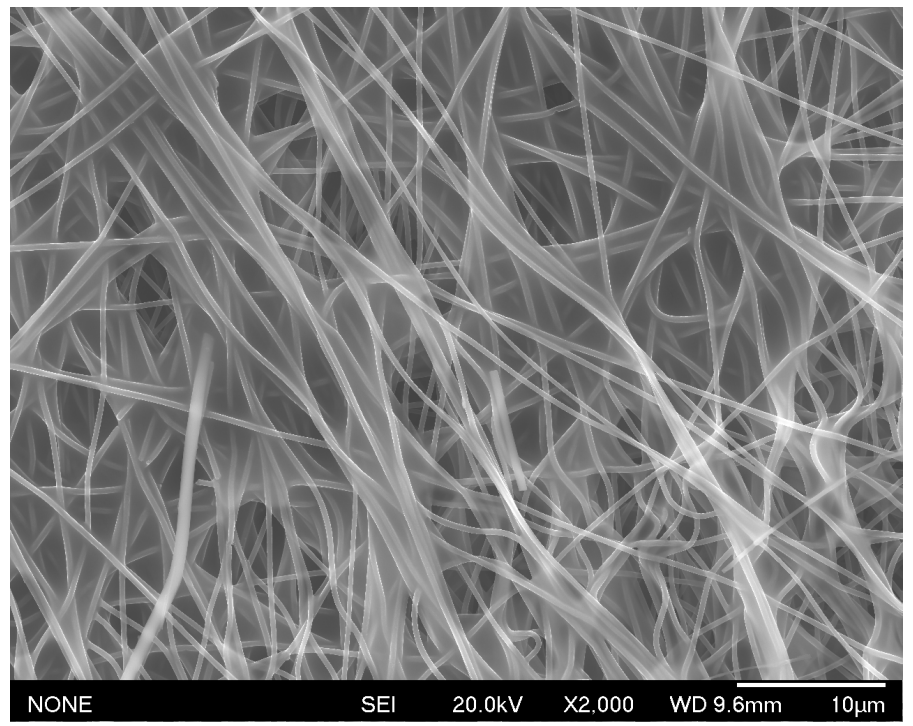

FIGURE 6.19: SEM micrograph of $\mathrm{C} / \mathrm{V}_{2} \mathrm{O}_{5}$ nanofibres after performing 20 chargedischarge cycles at $100 \mathrm{~mA} \mathrm{~g}^{-1}$.

An important consideration that can be made from these results is that, contrary to what previously assumed, the relatively low degree of graphitic character of CNFs (see also Figure 5.6) could be sufficient to allow reversible intercalation of $\mathrm{AlCl}_{4}^{-}$ions. However, this is only made possible by the presence of a sheath layer, which inhibits the early onset of electrolyte degradation and formation of an SEI by shielding the surface of CNFs from direct contact with the electrolyte. This could also be interpreted as further proof that the surface defects of CNFs are the main factor responsible to the early voltage onset of the degradation of the electrolyte, causing the formation of an SEI: the $\mathrm{V}_{2} \mathrm{O}_{5}$ layer suppresses the unwanted process by shielding the surface of CNFs from direct contact with the electrolyte. After a few cycles, however, the sheath layer is removed, exposing the carbon core and causing the disruption of the mixed-ion intercalation mechanism.

\subsection{Conclusions}

In summary, core-sheath $\mathrm{C} / \mathrm{V}_{2} \mathrm{O}_{5}$ nanofibres were successfully fabricated, and their performance in AIBs have been tested. Experimental evidence suggests that a mixed-ion mechanism, involving the "alternating" intercalation of both $\mathrm{Al}^{3+}$ 
and $\mathrm{AlCl}_{4}^{-}$, could be taking place in the material. This could be made possible by $\mathrm{V}_{2} \mathrm{O}_{5}$ layer inhibiting of the SEI formation process that would normally happen in CNF cathodes by limiting the contact between the surface defects of CNFs and the electrolyte. Because of this, the carbon core could allow the insertion of $\mathrm{AlCl}_{4}^{-}$ ions in the same way as graphitic materials do. This is a highly desirable feature, as such mechanism would maximise the energy density of the system by optimising the utilisation of the electrolyte. ${ }^{11,12}$ However, this synergistic behaviour is unfortunately short-lived, as the $\mathrm{V}_{2} \mathrm{O}_{5}$ sheath tends to disintegrate after a few cycles, exposing the carbon core and leading to the formation of SEI and overall poor performance. Perhaps further work could be performed in the future to find a material with a more stable behaviour, which could allow this mixed-ion intercalation mechanism over a large number of cycles.

\subsection{Experimental section}

All the chemicals mentioned in this section were purchased from Sigma-Aldrich and used without any additional treatment, unless differently specified. Al foil was purchased from MTI Corp.

SEM micrographs were acquired with a JEOL-JSM6500F field emission scanning electron microscope, using an accelerating voltage of $20 \mathrm{kV}$. In order to enhance imaging contrast, polymeric samples were sputter-coated with a $10 \mathrm{~nm}$ Pt layer.

TEM micrographs and STEM-EDX elemental maps were acquired with a JEOLJEM2100F transmission electron microscope, using an accelerating voltage of 200 $\mathrm{kV}$. Samples were prepared by dispersing the material in ethanol via ultrasonication and drop casting onto a lacey carbon grid.

For ex situ experiments, the cycled devices were disassembled inside the glovebox, and the cathodes were washed with ethanol to remove any residual electrolyte.

Thermogravimetric analysis was performed using a Shimadzu TGA-50 thermogravimetric analyser operating under a flow of $50 \mathrm{~mL} / \mathrm{min}$ of either air or nitrogen, using the following temperature programme: heating at $10^{\circ} \mathrm{C} /$ minute until $120{ }^{\circ} \mathrm{C}$, hold for 10 minutes to eliminate residual water in the sample, heating at $10^{\circ} \mathrm{C} /$ minute until the final temperature, and hold for another ten minutes 
X-ray diffractograms were acquired using a PANalytical X'pert PRO XRD diffractometer operating in reflection mode, using a $\mathrm{Cu} \mathrm{K} \alpha$ X-ray source, in the Bragg angle range between 10 and 80 degrees. Samples were prepared by lightly grinding the materials and depositing them on a zero-background holder.

XPS spectra were acquired using a Kratos Axis UltraDLD X-ray photoelectron spectrometer (Kratos Analytical, Manchester, UK) equipped with a hemispherical electron energy analyser. Spectra were excited using monochromatic $\mathrm{Al} \mathrm{K} \mathrm{K}_{\alpha}$ X-rays $(1486.69 \mathrm{eV})$ with the X-ray source operating at $150 \mathrm{~W}$. This instrument illuminates a large area on the surface and then using hybrid magnetic and electrostatic lenses collects photoelectrons from a desired location on the surface. In this case the analysis area was a 300 by $700 \mu \mathrm{m}$ spot obtained using the hybrid magnetic and electrostatic lens and the slot aperture. Measurements were carried out in a normal emission geometry. Core level scans were collected with a pass energy of $20 \mathrm{eV}$. The depth profile used a mini-beam III (MBIII) ion gun. A $5 \mathrm{kV} \mathrm{Ar+}$ ion beam was rastered over a $3 \mathrm{~mm} \times 3 \mathrm{~mm}$ area. To avoid etch crater wall effects, the depth profile data was collected using the FOV1 lens and the slot aperture approx. $220 \mu \mathrm{m}$ square (as opposed to the hybrid mode) The analysis chamber was at pressures in the $10^{-9}$ torr range throughout the data collection. Data analysis was performed using the CasaXPS software.

Cyclic voltammetry tests were performed using a Metrohm Autolab PGSTAT128N potentiostat, operating in a 2-electrode configuration: the cathode terminals of the devices were connected to the working electrode, and the anode terminals were connected to the counter and reference electrode.

Galvanostatic charge-discharge experiments were performed using a NEWARE BTS CT-4008-5V10mA-164 (MTI Corp.) battery analyser system.

\section{Fabrication of CNFs}

A precursor solution was prepared by adding $0.8 \mathrm{~mL}$ of PAN (average $\mathrm{M}_{w}$ : $150,000)$ to $10 \mathrm{~mL}$ of DMF under magnetic stirring. The solution was then loaded into a plastic syringe with a stainless steel, 18-gauge, blunt tip needle. The electrospinning process was then performed, using a voltage of $15 \mathrm{kV}$, a flow rate of 1 $\mathrm{mL} /$ hour, and a tip-to-collector distance of $13 \mathrm{~cm}$. A rotating drum collector was 
used in the process, to optimise the form factor of the nanofibre mats. The resulting fibres were first heat-treated in air using a muffle furnace with the following heating programme: heating at $5{ }^{\circ} \mathrm{C} /$ minute until $220^{\circ} \mathrm{C}$, holding the temperature for 2 hours, and cooling naturally to room temperature. Finally, the fibres were carbonised under a nitrogen atmosphere using a tube furnace with the following heating programme: heating at $4{ }^{\circ} \mathrm{C} /$ minute until $200{ }^{\circ} \mathrm{C}$, hold temperature for 20 minutes, heating at $10^{\circ} \mathrm{C} /$ minute until $600{ }^{\circ} \mathrm{C}$, hold temperature for 20 minutes, heating at $5{ }^{\circ} \mathrm{C} /$ minute until $1400{ }^{\circ} \mathrm{C}$, hold temperature for 1 hour, cooling at 5 ${ }^{\circ} \mathrm{C} /$ minute until $200{ }^{\circ} \mathrm{C}$, and finally cooling naturally to room temperature.

\section{Fabrication of core-sheath $\mathrm{C} / \mathrm{V}_{2} \mathrm{O}_{5}$ nanofibres}

Following the literature procedure by Li et al., ${ }^{5}$ a precursor solution was prepared by stirring $200 \mu \mathrm{L}$ of VOTIP into $30 \mathrm{~mL}$ of isopropanol. The solution was then transferred into a stainless steel autoclave with a $50 \mathrm{~mL}$ teflon-lined vessel, and a $3 \mathrm{~cm} \times 3 \mathrm{~cm}$ CNF sheet was then added. The autoclave was then sealed and heated at $200{ }^{\circ} \mathrm{C}$ for 10 hours. Then, the nanofibre sheet was removed from the solution, washed three times with water and ethanol, and dried in air. Finally, the nanofibres were annealed by heating them at $240^{\circ} \mathrm{C}$ overnight in a vacuum oven.

\section{Electrolyte preparation}

[EMIm] Cl and $\mathrm{AlCl}_{3}$ were baked at $100{ }^{\circ} \mathrm{C}$ in a vacuum oven for 48 hours to remove its water content, and immediately brought inside a nitrogen-filled glovebox with the $\mathrm{O}_{2}$ and $\mathrm{H}_{2} \mathrm{O}$ levels kept below $1 \mathrm{ppm}$. The electrolyte was prepared by gradually adding 1.3 equivalents of anhydrous $\mathrm{AlCl}_{3}$ to 1 equivalent [EMIm] Cl inside the aforementioned glovebox. An exothermic reaction takes place, after which a brown, slightly viscous liquid is formed.

\section{Device construction}

Nanofibre mats were cut into $11 \mathrm{~mm}$ diameter discs to be used as cathode in the devices. Prototype batteries were then assembled using a custom-built Swageloktype cell consisting of a cylindrical PEEK casing with an inner diameter of 12 $\mathrm{mm}$ and two Mo rods as the current collectors (see also Section 3.4.2). All the 
components of the device were first baked at $100{ }^{\circ} \mathrm{C}$ in a vacuum oven for at least 2 hours to remove any residual water, then immediately transferred inside a nitrogen-filled glovebox with the $\mathrm{O}_{2}$ and $\mathrm{H}_{2} \mathrm{O}$ levels kept below $1 \mathrm{ppm}$. One of the previously prepared NF discs was used as a cathode, and $11 \mathrm{~mm}$ diameter, high purity $\mathrm{Al}$ foil discs were used as the anode. Glass microfibre (GF/D) discs with $12 \mathrm{~mm}$ diameters, soaked in approximately $300 \mu \mathrm{L}$ of the electrolyte, were used as separators. The devices were then wrapped with Parafilm as an additional moisture barrier, and taken outside the glovebox for electrochemical testing.

\section{Bibliography}

${ }^{1}$ M. Chiku, H. Takeda, S. Matsumura, E. Higuchi, and H. Inoue, "Amorphous Vanadium Oxide/Carbon Composite Positive Electrode for Rechargeable Aluminum Battery," ACS Applied Materials \& Interfaces, vol. 7, pp. 24385-24389, Nov. 2015.

${ }^{2}$ A. Witkowski, A. A. Stec, and T. R. Hull, "Thermal Decomposition of Polymeric Materials," in SFPE Handbook of Fire Protection Engineering (M. J. Hurley, D. Gottuk, J. R. Hall, K. Harada, E. Kuligowski, M. Puchovsky, J. Torero, J. M. Watts, and C. Wieczorek, eds.), pp. 167-254, New York, NY: Springer New York, 2016.

${ }^{3}$ C. J. Brinker, G. C. Frye, A. J. Hurd, and C. S. Ashley, "Fundamentals of sol-gel dip coating," Thin Solid Films, vol. 201, pp. 97-108, June 1991.

${ }^{4}$ W. Wang, B. Jiang, W. Xiong, H. Sun, Z. Lin, L. Hu, J. Tu, J. Hou, H. Zhu, and S. Jiao, “A new cathode material for super-valent battery based on aluminium ion intercalation and deintercalation," Scientific Reports, vol. 3, Nov. 2013.

${ }^{5}$ L. Li, S. Peng, H. B. Wu, L. Yu, S. Madhavi, and X. W. D. Lou, "A Flexible Quasi-Solid-State Asymmetric Electrochemical Capacitor Based on Hierarchical Porous V2O5 Nanosheets on Carbon Nanofibers," Advanced Energy Materials, vol. 5, p. 1500753, Sept. 2015.

${ }^{6}$ Y. Wang and G. Cao, "Synthesis and Enhanced Intercalation Properties of Nanostructured Vanadium Oxides," Chemistry of Materials, vol. 18, pp. 2787-2804, June 2006.

${ }^{7}$ G. Li, S. Pang, L. Jiang, Z. Guo, and Z. Zhang, "Environmentally Friendly Chemical Route to Vanadium Oxide Single-Crystalline Nanobelts as a Cathode Material for Lithium-Ion Batteries," The Journal of Physical Chemistry B, vol. 110, pp. 9383-9386, May 2006.

${ }^{8}$ C. Zhou, L. Mai, Y. Liu, Y. Qi, Y. Dai, and W. Chen, "Synthesis and Field Emission Property of V2O5.nH2O Nanotube Arrays," The Journal of Physical Chemistry C, vol. 111, pp. 8202-8205, June 2007.

${ }^{9}$ Y. Yang, Q. Zhu, A. Jin, and W. Chen, "High capacity and contrast of electrochromic tungstendoped vanadium oxide films," Solid State Ionics, vol. 179, pp. 1250-1255, Sept. 2008.

${ }^{10} \mathrm{~S}$. Gu, H. Wang, C. Wu, Y. Bai, H. Li, and F. Wu, “Confirming reversible Al3+ storage mechanism through intercalation of $\mathrm{Al} 3+$ into $\mathrm{V} 2 \mathrm{O} 5$ nanowires in a rechargeable aluminum battery," Energy Storage Materials, vol. 6, pp. 9-17, Jan. 2017.

${ }^{11}$ D. J. Kim, D.-J. Yoo, M. T. Otley, A. Prokofjevs, C. Pezzato, M. Owczarek, S. J. Lee, J. W. Choi, and J. F. Stoddart, "Rechargeable aluminium organic batteries," Nature Energy, p. 1, Dec. 2018. 
${ }^{12}$ Y. Liang and Y. Yao, "Halfway through," Nature Energy, p. 1, Dec. 2018.

${ }^{13}$ N. Jayaprakash, S. K. Das, and L. A. Archer, "The rechargeable aluminum-ion battery," Chemical Communications, vol. 47, no. 47, pp. 12610-12612, 2011.

${ }^{14}$ H. Wang, Y. Bai, S. Chen, X. Luo, C. Wu, F. Wu, J. Lu, and K. Amine, "Binder-Free V2O5 Cathode for Greener Rechargeable Aluminum Battery," ACS Applied Materials \& Interfaces, vol. 7, pp. 8084, Jan. 2015.

${ }^{15}$ J. F. Moulder, W. F. Stickle, P. E. Sobol, and K. D. Bomben, Handbook of X Ray Photoelectron Spectroscopy. Eden Prairie, Minn: Physical Electronics, Feb. 1993. 

Chapter 7

Cathode materials: layered nanostructured electrode 



\subsection{Preface}

This avenue of research was originally conceived as an alternative route to combine the properties of carbon and vanadium oxide. Given the initial difficulties encountered trying to create a core-sheath material to use as a cathode, a different strategy adopted at the same time. The rationale for this design consists of preparing the nanomaterials separately and then assembling them in a functional structure in a later step. This way, any incompatibilities from the need to perform heat treatments under both oxidising and inert atmospheres are removed. With this reasoning in mind, a new material was conceptualised: a cathode composed of nanostructured $\mathrm{V}_{2} \mathrm{O}_{5}$ with a high surface area supported on a carbon nanofibre bottom layer, which would act as a current collector. Although preliminary experiments (see also Chapter 5) suggested that non-graphitic CNFs would not show any significant activity as a cathode for AIBs, their interesting mechanical properties and conductivity could allow them to work as a nanostructured current collector, onto which an active material could be placed. Therefore, a layer of nanostructured $\mathrm{V}_{2} \mathrm{O}_{5}$ deposited directly on top of a CNF sheet could potentially work as a cathode for AIBs. In addition, another layer consisting of polymeric nanofibres could be directly electrospun on top of the active layer, which could both act as the separator in the battery device and as a means to keep the underlying components in place and preventing from disintegrating or diffusing into the device (Figure 7.1).

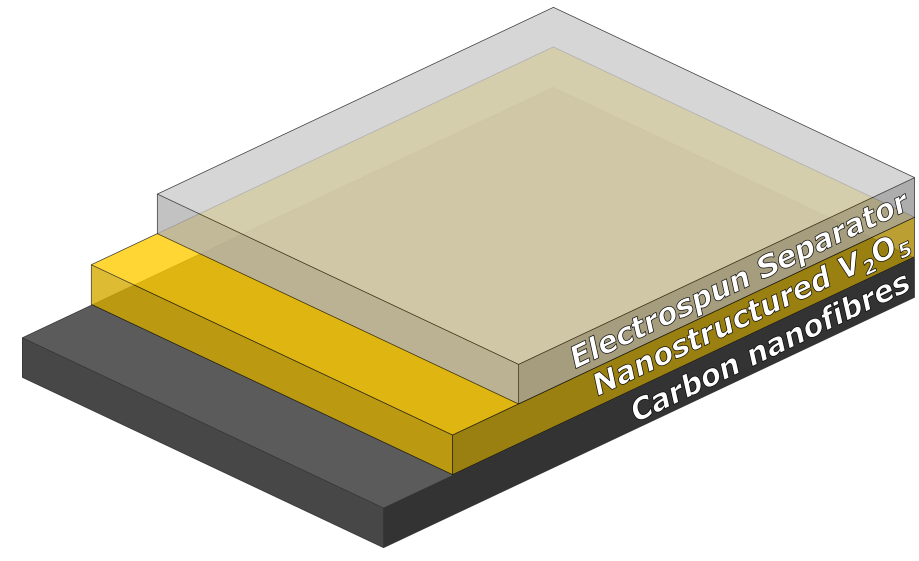

FIGURE 7.1: Schematic representation of the layered nanostructured electrode. 
Furthermore, by using this method, the the three layers can be prepared by individual processes, with minimal interference between them. CNFs can be synthesised using the previously consolidated procedure and then nanostructured $\mathrm{V}_{2} \mathrm{O}_{5}$ can be prepared separately and deposited by a simple drop casting procedure or analogues. Finally, the separator can be directly electrospun onto the resulting material. The material could then, in theory, be used directly in a device without any further treatment.

\subsection{Electrode fabrication}

\subsubsection{Nanostructured $\mathrm{V}_{2} \mathrm{O}_{5}$ layer}

Because CNFs can be easily prepared with our previously established method, the first step in this project consisted of choosing an adequate type of nanostructured $\mathrm{V}_{2} \mathrm{O}_{5}$ to be used as the active material for the electrode. Although a protocol for the fabrication of $\mathrm{V}_{2} \mathrm{O}_{5}$ nanofibres was developed as part of this $\mathrm{PhD}$ (see also Chapter 4), and a number of publications reporting various nanostructured $\mathrm{V}_{2} \mathrm{O}_{5}$ materials are available in recent literature, ${ }^{1}$ it was decided to opt for a different option. Previous attempts to fabricate core-sheath $\mathrm{C} / \mathrm{V}_{2} \mathrm{O}_{5}$ nanofibres according to the procedure described by $\mathrm{Li}$ et al. ${ }^{2}$ (see also Chapter 6), revealed that during the solvothermal process, the $\mathrm{V}_{2} \mathrm{O}_{5} \cdot \mathrm{xH}_{2} \mathrm{O}$ nanosheets would grow not only on the CNF substrate, but also on the walls of the PTFE vessel as the formation of round clusters consisting of crumpled $\mathrm{V}_{2} \mathrm{O}_{5} \cdot \mathrm{xH}_{2} \mathrm{O}$ sheets was observed. It was hypothesised that this kind of nanostructure could be an ideal candidate as the active material for our electrode, thanks to its high surface area and small feature size, which would potentially allow it to reliably deposit on the CNF mat. This could possibly even allow the sheets to partially permeate through the mesoscopic pores of the substrate, leading to a higher contact surface between the current collector and the active material. Therefore, the same solvothermal process described in the previous chapter was then used, but without the addition of a sheet of CNFs inside the reaction vessel. This way, the nanomaterials only grew in the solution and on the walls of the PTFE vessel, and were easily recovered by removal of the solvent via rotary evaporation. The resulting material was then annealed in air at $300{ }^{\circ} \mathrm{C}$ for 1 hour to yield pure $\mathrm{V}_{2} \mathrm{O}_{5}$. The final morphology of the nanomaterial 


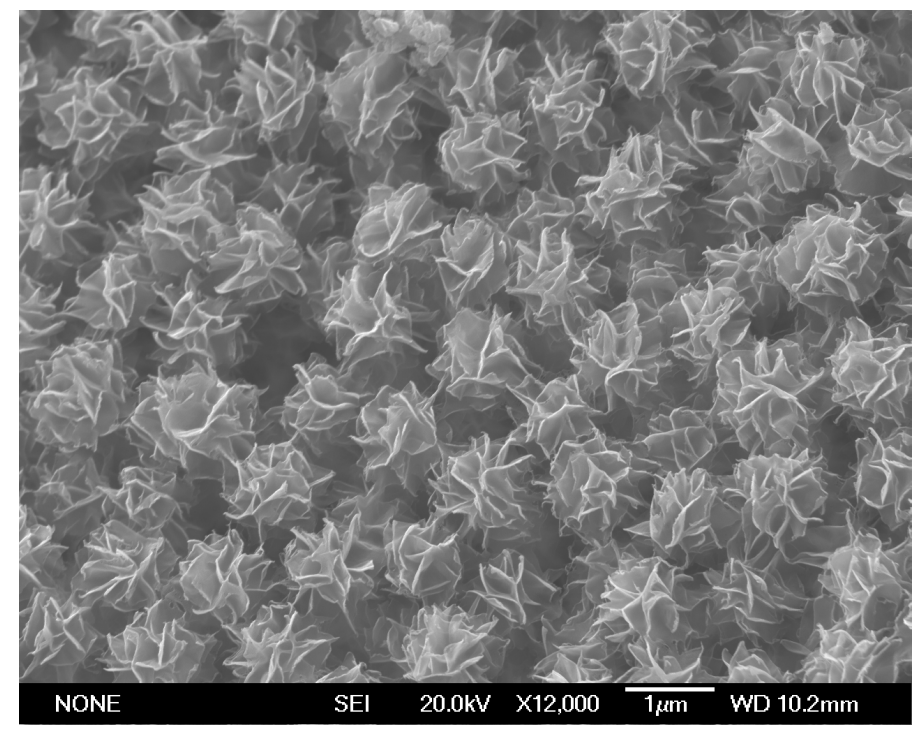

FIGURE 7.2: SEM micrograph of $\mathrm{V}_{2} \mathrm{O}_{5}$ nanoflowers obtained by solvothermal growth.

consists in flower-like round structures of about $1 \mu \mathrm{m}$ diameter, made of individual "sheets", slightly crumpled and fused together (Figure 7.2). Interestingly, similar structures were obtained by another publication, ${ }^{3}$ albeit using a completely different procedure and precursors; the authors used the term "nanoflowers" to describe such morphology. For the sake of simplicity, the same terminology will be used herein.

It can be seen from the SEM image that individual nanoflowers are fused together into larger structures. Therefore, in order to make the material fit for deposition, the nanoflowers were first dispersed in dichloromethane then ultrasonicated for 30 minutes to separate the individual nanoflowers as best as possible. The resulting yellow coloured suspension appeared consistent over a few days, indicating the metastability of the dispersion.

Spin coating was chosen as the deposition technique for the formation of the active $\mathrm{V}_{2} \mathrm{O}_{5}$ nanoflower layer. ${ }^{4}$ The main reason for this choice is that this technique allows for a more uniform coating of the substrate. Furthermore, it was hypothesised that the more conventional doctor blading method would possibly not be a suitable option in this case, as the mechanical properties of the CNF substrate would not allow a reliable casting of the electrode slurry. In addition, the nanofibre sheet could even be damaged by the shearing forces exerted by the blade 

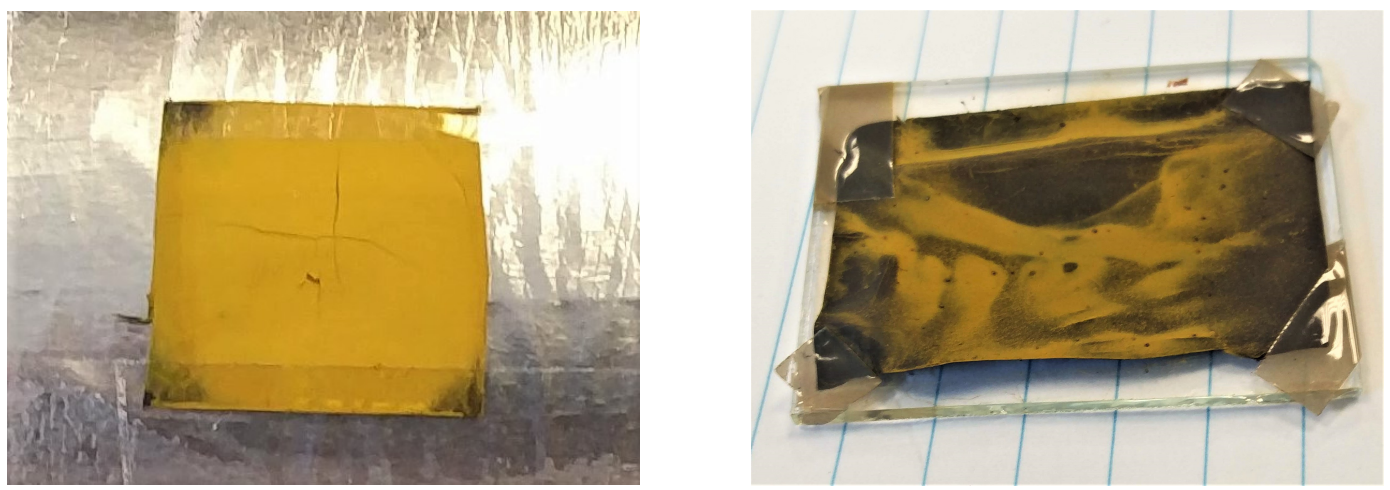

FIGURE 7.3: Digital camera images displaying the issues encountered while depositing $\mathrm{V}_{2} \mathrm{O}_{5}$ nanoflowers on CNFs by spin coating: formation of cracks (left) and uneven deposition (right).

while spreading the film, as the technique is usually performed on more rigid substrates such as metal or glass. The $\mathrm{V}_{2} \mathrm{O}_{5}$ nanoflower suspension was therefore spin-coated onto a small sheet of CNFs to create the active layer of the electrode. Some difficulties were initially encountered; due to the flexibility of CNFs, the substrate tended to pucker into a concave shape as a consequence of the vacuum used to adhere the substrate to the spin coater and relax again to a flat sheet once the vacuum was removed. This led to the formation of cracks in the deposited layer (Figure 7.3 (left)). The issue was solved by attaching the CNF sheet to a glass slide using adhesive tape on the edges of the mat. Another issue that was encountered is the fact that CNF sheets are often inherently slightly uneven and wrinkled. Because of this, the deposited material tended to accumulate in certain areas, and leave other areas uncoated (Figure 7.3 (right)). This problem was somewhat mitigated by optimising the fabrication of CNFs to minimise the formation of wrinkled surfaces, and selecting the flattest portion of the CNF sheet to use in the process. Despite taking such precautions, however, some degree of irregularity in the thickness of the $\mathrm{V}_{2} \mathrm{O}_{5}$ layer can always be expected to be present. Finally, the spin coating conditions (static or dynamic casting, material concentration in the casting solution, casting volume, spinning speed and time) also needed to be optimised to obtain acceptable films. After a few attempts, an acceptable $\mathrm{CNF} / \mathrm{V}_{2} \mathrm{O}_{5}$ composite was finally obtained. 


\subsubsection{Electrospun separator}

For the final step, polymeric nanofibres needed to be electrospun on top of the $\mathrm{V}_{2} \mathrm{O}_{5}$ layer to act as both as a sealing layer and as a separator. This was performed by attaching the $\mathrm{CNF} / \mathrm{V}_{2} \mathrm{O}_{5}$ composite to the rotating drum collector of the electrospinning setup with adhesive tape and electrospinning a polymeric solution for about 3 hours. Polyacrylonitrile was chosen as the polymer to be electrospun, as a recent publication reported the possibility of using the material to fabricate high performance separators. ${ }^{5}$ This step was relatively easy to perform: after the electrospinning process, the electrode was removed from the nanofibre collector using a scalpel blade, and a three-layered structure was readily obtained. SEM imaging was performed on the finished material to confirm its layered structure (Figure 7.4).

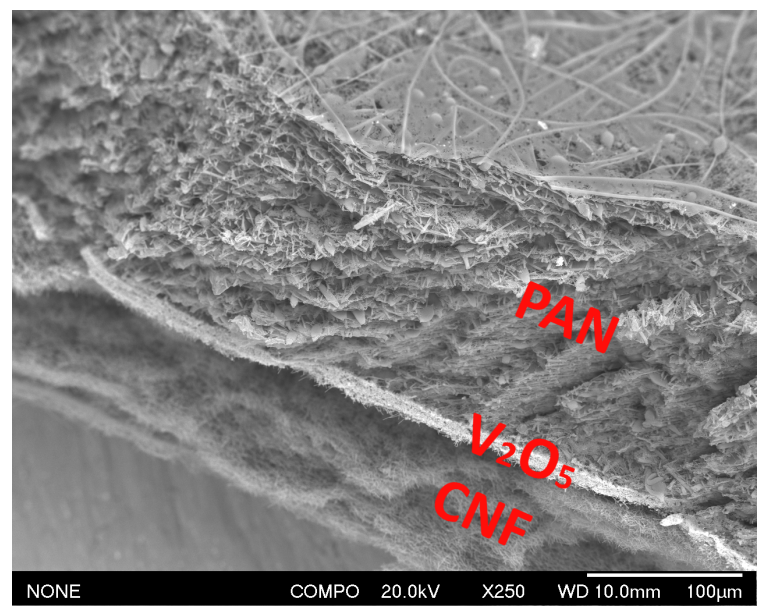

FIGURE 7.4: SEM micrograph (using the back-scattered electron detector) of a cross-section of the finished layered nanostructured electrode.

\subsection{Battery tests}

\subsubsection{Device assembly}

In order to be used in a device, the electrode needed to be cut into appropriately sized discs. This however proved to be very difficult due to the tendency of the layers to separate, rendering the material unusable. The reason for this phenomenon is likely due to the poor adherence of the $\mathrm{V}_{2} \mathrm{O}_{5}$ layer to both the PAN and CNF layers. This can be somewhat seen as a predictable design flaw 
in the material; any layer thicker than a "monolayer" of $\mathrm{V}_{2} \mathrm{O}_{5}$ nanoflowers would likely act as a free-flowing powder, which therefore would not allow the adhesion of the other layers. This could be potentially remedied by using a more conventional approach to the deposition such as processing the $\mathrm{V}_{2} \mathrm{O}_{5}$ nanoflowers with a binder polymer. This modification, however, would then possibly require that the material is cast onto the substrate by doctor blading, as the resulting dispersion would have a very high viscosity, which is unsuitable for spin coating. Therefore, a different strategy was chosen: instead of electrospinning the PAN layer directly on top of the $\mathrm{V}_{2} \mathrm{O}_{5}$ layer, a sheet of PAN nanofibres was independently fabricated. Then, the PAN nanofibre sheet and the $\mathrm{CNF} / \mathrm{V}_{2} \mathrm{O}_{5}$ composite were cut into discs separately, and assembled together during the fabrication of the device. Although this method is more akin to conventional device fabrication, there are still elements of novelty, such as using CNFs as the current collector, and not using any binder polymer or conductivity enhancer in combination with the the active material. Furthermore, this method allowed the PAN separators to be cut into discs with larger diameters than the positive and negative electrodes. This is a more desirable cell design, as it prevents short circuiting between the anode and the cathode more effectively.

\subsubsection{Electrochemical tests}

After assembling the prototype devices, galvanostatic charge-discharge experiments were performed using similar parameters to the ones used for the other $\mathrm{V}_{2} \mathrm{O}_{5}$-based batteries. One important difference between this device and the ones assembled for previous projects is worth noting. The determination of the mass of active material, from which the values of specific capacity and cycling rates are calculated (see also Section 3.4.3), is not easy to measure accurately due to the nature of the fabrication process. To determine the quantity of active material, the absolute mass of $\mathrm{V}_{2} \mathrm{O}_{5}$ on a full-sized $\mathrm{CNF} / \mathrm{V}_{2} \mathrm{O}_{5}$ composite sheet is measured by weighing the CNF mats before and after the spin coating process, and subtracting the respective values. From these measurements, the mass fraction of $\mathrm{V}_{2} \mathrm{O}_{5}$ in the electrode can be determined. After the electrodes are cut into discs, the mass of active material can be calculated by weighing the cut electrode and use the mass fraction to derive the mass of active material. This calculation, however, is made 
under the assumption that the mass distribution of $\mathrm{V}_{2} \mathrm{O}_{5}$ is constant across the surface of the $\mathrm{CNF} / \mathrm{V}_{2} \mathrm{O}_{5}$ composite. Because of the variability of the thickness of the $\mathrm{V}_{2} \mathrm{O}_{5}$ layer, this assumption can often be inaccurate. The performance metrics reported in this chapter should therefore be considered as approximate as they are all derived using the mass of active material.

A protocol of 20 cycles, starting with the discharging phase, was performed on the device. Because this test served mainly as a proof-of-concept, a slow chargedischarge rate of approximately $20 \mathrm{~mA} \mathrm{~g}^{-1}$ was used. Although the first tests were performed in the range of 2.45 to $0.02 \mathrm{~V}$, a large loss of coulombic efficiency was noted, similarly to what was observed for CNF cathodes (see also Section 5.2.2), which is likely due to the formation of a SEI at the interface with the CNF layer. Therefore, in order to maintain an acceptable coulombic efficiency, the upper voltage cutoff was lowered to $2.27 \mathrm{~V}$. Unfortunately, the performance of the device proved to be very unstable, and only ten full charge-discharge cycles could be obtained before the failure of the device. The results of the test are shown in Figure 7.5.
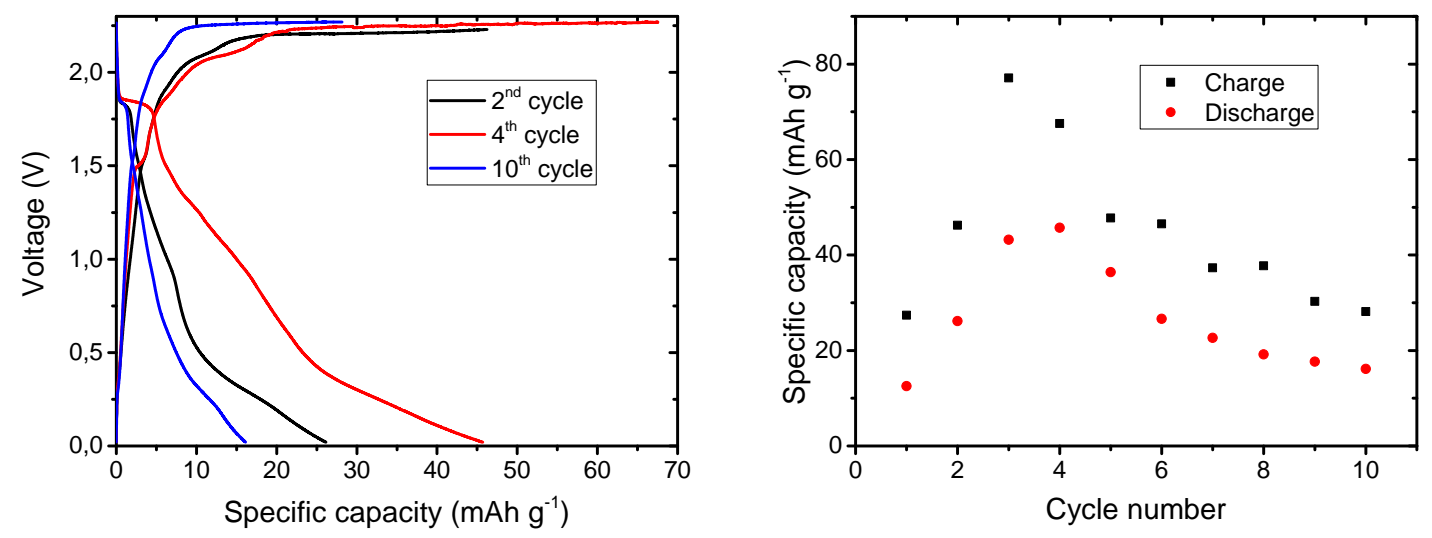

FIGURE 7.5: Galvanostatic charge-discharge data of a Swagelok-type cell built using the layered nanostructured electrode: charge-discharge profiles of the second, fourth, and tenth cycles (left) and specific capacity data (right). The voltage reported in this plot is the cell potential.

It can be seen that the electrode yields a galvanostatic profile that bears some similarities to the ones previously observed for $\mathrm{V}_{2} \mathrm{O}_{5}$-based cathodes (Figure 4.6): one wide plateau when discharging below $0.5 \mathrm{~V}$ and one in the charging profile beyond $2 \mathrm{~V} \cdot 6,7$ Interestingly, some different features can also be seen. Namely, a small discharge plateau at about $1.8 \mathrm{~V}$ is observed and gets more prominent towards the 
fourth cycle before fading in the subsequent cycles. In the charging profile, the presence of very faint plateaus between 1.5 and $2.1 \mathrm{~V}$ can also be observed; these also appear to be most pronounced in the fourth cycle. Furthermore, contrarily to what was observed for the $\mathrm{V}_{2} \mathrm{O}_{5} \mathrm{NF}$ cathode, the charging capacity is higher than the discharging capacity. This is likely due to the participation of the CNF layer in the electrochemical processes, either by exhibiting capacitive/pseudo-capacitive behaviour or promoting the degradation of the electrolyte and the formation of the SEI. It is worth noting that the synergistic effect between the CNFs and $\mathrm{V}_{2} \mathrm{O}_{5}$ in the core-sheath nanofibres (see also Section 6.3.1) is not present in these composite electrodes. This is likely due to the fact that in this material, the surface of the CNFs is not effectively coated by the $\mathrm{V}_{2} \mathrm{O}_{5}$ nanosheets, and is therefore in direct contact with the electrolyte. The decomposition of the electrolyte and formation of SEI is likely still taking place, hindering the intercalation of $\mathrm{AlCl}_{4}^{-}$into the CNFs, similarly to what was observed in Chapter 5.

The test also reveals that both the charging and discharging specific capacities tend to first increase until the third / fourth cycle, and then gradually decrease until the failure of the device. This behaviour is not easily interpreted, but a possible explanation for the high instability of the device could be due to the electrode gradually disintegrating. Because the active material is not effectively anchored but only loosely deposited on the CNF-based current collector, it is quite possible that it would gradually displace, and eventually completely detach from the current collector, leading to an open circuit and the failure of the device.

\subsection{Conclusions}

The tests performed on this material reveal that although a working device can be built using the composite electrode as cathode, no reliable cycling performance can be obtained. In fact, the high fragility of the electrode only allows for a very short device life, as the discharge capacity rapidly decreases due to the disintegration of the material. Furthermore, no significant difference in specific discharge capacity was observed between this electrode and the ones built with conventional methods in Chapter 4. Therefore, it can be concluded that the electrode structure and fabrication methods described in this chapter are not ideal for the intended application, and simpler electrode fabrication techniques such as those 
used in Chapter 4, or even the nanostructure and methods described in Chapter 6 are preferable, thanks to their lower complexity, and better overall performance in AIB devices.

\subsection{Experimental section}

All the chemicals mentioned in this section were purchased from Sigma-Aldrich and used without any additional treatment, unless differently specified. Al foil was purchased from MTI Corp.

SEM micrographs were acquired with a JEOL-JSM6500F field emission scanning electron microscope, using an accelerating voltage of $20 \mathrm{kV}$. In order to enhance imaging contrast, samples were sputter-coated with a $10 \mathrm{~nm}$ Pt layer.

Galvanostatic charge-discharge experiments were performed using a NEWARE BTS CT-4008-5V10mA-164 (MTI Corp.) battery analyser system.

\section{Fabrication of CNFs}

A precursor solution was prepared by adding $0.8 \mathrm{~mL}$ of PAN (average $\mathrm{M}_{w}$ : $150,000)$ to $10 \mathrm{~mL}$ of DMF under magnetic stirring. The solution was then loaded into a plastic syringe with a stainless steel, 18-gauge, blunt tip needle. The electrospinning process was then performed, using a voltage of $15 \mathrm{kV}$, a flow rate of 1 $\mathrm{mL} /$ hour, and a tip-to-collector distance of $13 \mathrm{~cm}$. A rotating drum collector was used in the process, to optimise the form factor of the nanofibre mats. The resulting fibres were first heat-treated in air using a muffle furnace with the following heating programme: heating at $5{ }^{\circ} \mathrm{C} /$ minute until $220^{\circ} \mathrm{C}$, holding the temperature for 2 hours, and cooling naturally to room temperature. Finally, the fibres were carbonised under a nitrogen atmosphere using a tube furnace with the following heating programme: heating at $4{ }^{\circ} \mathrm{C} /$ minute until $200{ }^{\circ} \mathrm{C}$, hold temperature for 20 minutes, heating at $10{ }^{\circ} \mathrm{C} /$ minute until $600{ }^{\circ} \mathrm{C}$, hold temperature for 20 minutes, heating at $5{ }^{\circ} \mathrm{C} /$ minute until $1400{ }^{\circ} \mathrm{C}$, hold temperature for 1 hour, cooling at 5 ${ }^{\circ} \mathrm{C} /$ minute until $200{ }^{\circ} \mathrm{C}$, and finally cooling naturally to room temperature. 


\section{Fabrication of $\mathrm{V}_{2} \mathrm{O}_{5}$ nanoflowers}

A solution was prepared by stirring $200 \mu \mathrm{L}$ of VOTIP into $30 \mathrm{~mL}$ of isopropanol. This was then transferred into a stainless steel autoclave with a $50 \mathrm{~mL}$ teflonlined vessel, which was then sealed and heated at $200{ }^{\circ} \mathrm{C}$ for 10 hours. After the heat treatment, the solution and the resulting dark precipitate were transferred into a round-bottom flask, and the solvent was removed by rotary evaporation. The precipitate was then washed with de-ionised water to remove any unreacted vanadium precursor, and dried in air. Finally, product was annealed in air, using a muffle furnace, performing the following heating programme: heating at 5 ${ }^{\circ} \mathrm{C} /$ minute until $300{ }^{\circ} \mathrm{C}$, hold temperature for 1 hour, and natural cooling.

\section{Fabrication of $\mathrm{C}-\mathrm{V}_{2} \mathrm{O}_{5}$ composite}

Approximately $200 \mathrm{mg}$ of $\mathrm{V}_{2} \mathrm{O}_{5}$ nanoflowers were dispersed in $10 \mathrm{~mL}$ of DCM using tip ultrasonication for approximately 30 minutes. A $3 \mathrm{~cm} \times 3 \mathrm{~cm} C N F$ sheet was attached to a glass slide using adhesive tape, which was then placed on a spin coater. The substrate was first washed with water and ethanol, then the active $\mathrm{V}_{2} \mathrm{O}_{5}$ layer was fabricated by depositing roughly $3 \mathrm{~mL}$ of the dichloromethanedispersed $\mathrm{V}_{2} \mathrm{O}_{5}$ nanoflowers drop-wise while, spinning the substrate at $800 \mathrm{rpm}$. The drop-wise coating was performed over a 20 second period, after which the substrate was left spinning at $800 \mathrm{rpm}$ for an additional 40 seconds to allow full evaporation of the solvent.

\section{Fabrication of PAN separator}

A precursor solution was prepared by adding $0.8 \mathrm{~mL}$ of PAN (average $\mathrm{M}_{w}$ : $150,000)$ to $10 \mathrm{~mL}$ of DMF under magnetic stirring. The solution was then loaded into a plastic syringe with a stainless steel, 18-gauge, blunt tip needle. The electrospinning process was then performed, using a voltage of $15 \mathrm{kV}$, a flow rate of 1 $\mathrm{mL} /$ hour, and a tip-to-collector distance of $13 \mathrm{~cm}$. A rotating drum collector was used in the process. The resulting nanofibre sheets were removed from the rotating drum collector, cut into $12 \mathrm{~mm}$ discs and used as separators in the devices. 


\section{Electrolyte preparation}

[EMIm]Cl and $\mathrm{AlCl}_{3}$ were baked at $100{ }^{\circ} \mathrm{C}$ in a vacuum oven for 48 hours to remove its water content, and immediately brought inside a nitrogen-filled glovebox with the $\mathrm{O}_{2}$ and $\mathrm{H}_{2} \mathrm{O}$ levels kept below $1 \mathrm{ppm}$. The electrolyte was prepared by gradually adding 1.3 equivalents of anhydrous $\mathrm{AlCl}_{3}$ to 1 equivalent [EMIm] Cl inside the aforementioned glovebox under magnetic stirring. An exothermic reaction took place, after which a brown, slightly viscous liquid is formed.

\section{Device construction}

$\mathrm{CNF} / \mathrm{V}_{2} \mathrm{O}_{5}$ composites were cut into $11 \mathrm{~mm}$ diameter discs to be used as cathode in the devices. Prototype batteries were then assembled using a custom-built Swagelok-type cell consisting of a cylindrical PEEK casing with an inner diameter of $12 \mathrm{~mm}$ and two Mo rods as the current collectors (see also Section 3.4.2). All the components of the device were first baked at $100{ }^{\circ} \mathrm{C}$ in a vacuum oven for at least 2 hours to remove any residual water, then immediately transferred inside a nitrogen-filled glovebox with the $\mathrm{O}_{2}$ and $\mathrm{H}_{2} \mathrm{O}$ levels kept below $1 \mathrm{ppm}$. One of the previously prepared $\mathrm{CNF} / \mathrm{V}_{2} \mathrm{O}_{5}$ composite discs was used as a cathode, and $11 \mathrm{~mm}$ diameter, high purity $\mathrm{Al}$ foil discs were used as the anode. PAN nanofibre discs with $12 \mathrm{~mm}$ diameters, soaked in approximately $300 \mu \mathrm{L}$ of the electrolyte, were used as separators. The devices were then wrapped with Parafilm as an additional moisture barrier, and taken outside the glovebox for electrochemical testing. 


\section{Bibliography}

${ }^{1}$ X. Liu, J. Zeng, H. Yang, K. Zhou, and D. Pan, "V2O5-Based nanomaterials: synthesis and their applications," RSC Advances, vol. 8, pp. 4014-4031, Jan. 2018.

${ }^{2}$ L. Li, S. Peng, H. B. Wu, L. Yu, S. Madhavi, and X. W. D. Lou, "A Flexible Quasi-Solid-State Asymmetric Electrochemical Capacitor Based on Hierarchical Porous V2O5 Nanosheets on Carbon Nanofibers," Advanced Energy Materials, vol. 5, p. 1500753, Sept. 2015.

${ }^{3}$ J. Mu, J. Wang, J. Hao, P. Cao, S. Zhao, W. Zeng, B. Miao, and S. Xu, "Hydrothermal synthesis and electrochemical properties of V2O5 nanomaterials with different dimensions," Ceramics International, vol. 41, pp. 12626-12632, Dec. 2015.

${ }^{4}$ D. B. Hall, P. Underhill, and J. M. Torkelson, "Spin coating of thin and ultrathin polymer films," Polymer Engineering \& Science, vol. 38, no. 12, pp. 2039-2045, 1998.

${ }^{5}$ G. A. Elia, J.-B. Ducros, D. Sotta, V. Delhorbe, A. Brun, K. Marquardt, and R. Hahn, "Polyacrylonitrile Separator for High-Performance Aluminum Batteries with Improved Interface Stability," ACS Applied Materials \& Interfaces, vol. 9, pp. 38381-38389, Nov. 2017.

${ }^{6}$ N. Jayaprakash, S. K. Das, and L. A. Archer, "The rechargeable aluminum-ion battery," Chemical Communications, vol. 47, no. 47, pp. 12610-12612, 2011.

${ }^{7}$ H. Wang, Y. Bai, S. Chen, X. Luo, C. Wu, F. Wu, J. Lu, and K. Amine, “Binder-Free V2O5 Cathode for Greener Rechargeable Aluminum Battery," ACS Applied Materials \& Interfaces, vol. 7, pp. 8084, Jan. 2015. 
Chapter 8

Novel electrolytes: amides/A1Cl 3 eutectic mixtures 



\subsection{Preface}

State-of-the-art AIBs currently rely on an electrolyte composed of a mixture of aluminium trichloride $\left(\mathrm{AlCl}_{3}\right)$ and 1-ethyl-3-methylimidazolium chloride ([EMIm] $\mathrm{Cl}$ ), an expensive, low-melting point organic salt. When these two components are mixed in a $\mathrm{AlCl}_{3}:[\mathrm{EMIm}] \mathrm{Cl}$ molar ratio greater than one, the resulting mixture is a room temperature ionic liquid with Lewis acid characteristics. This electrolyte enables the reversible electrodeposition of metallic aluminium on the anode, ${ }^{1}$ and the intercalation of either $\mathrm{AlCl}^{4-}$ or $\mathrm{Al}^{3+}$ ions in the cathode., ${ }^{2,3}$ Because all the other components of an AIB can be produced inexpensively from earth-abundant elements such as carbon, aluminium, and chlorine, the high cost of [EMIm] Cl is arguably one of the biggest drawbacks to the entire technology of AIBs, as in its current stage, the electrolyte easily constitutes its most expensive component. Therefore, in order to make AIBs more appealing and competitive with currently commercially available battery technologies, it is very important to find a low-cost alternative to [EMIm] $\mathrm{Cl}$ to be used in the electrolyte. In recent years, a number of inexpensive compounds have been proposed as an replacement for [EMIm] $\mathrm{Cl}$, with the aim to reduce the overall production costs of AIBs. These compounds include dipropylsulfone/toluene, ${ }^{4}$ urea, ${ }^{5}$ and inorganic salts such as $\mathrm{NaCl} .{ }^{6} \mathrm{Most}$ of the suggested alternatives, however, present critical issues such as requiring harsh working conditions (i.e. temperatures above $100^{\circ} \mathrm{C}$ ), or resulting in inferior performance. Therefore, the search for a suitable low-cost electrolyte for AIBs is still a very timely issue, which must be thoroughly addressed as a fundamental step towards the commercialisation of the technology.

Parts of this chapter were conducted in collaboration with Stuart Oliver, an undergraduate student at this university undertaking a short research project, and Nicolas Bertrand, an undergrad student from the SIGMA Clermont university in Aubière (France), who performed part of the work reported below as part of an internship at this university. Nicolas Bertrand's contribution consisted in the preparation of the acetamide-based electrolytes and their testing in a three-electrode cell configuration; Stuart Oliver's contribution consisted of testing some of the the diluting solvents for the acetamide-based electrolyte and part of the electrochemical tests in Swagelok-type cells. Part of the research described in this chapter (Sections 
8.2.2, 8.2.3, 8.2.4, and Figure A.19) was also published in a Chemical Communications article, released in September $2018 .^{7}$

\subsection{Acetamide-based electrolytes}

\subsubsection{The search for alternative compounds}

This project started with a literature research for compounds that were known to form eutectic mixtures with $\mathrm{AlCl}_{3}$, which is a fundamental prerequisite for them to be used as electrolytes for AIBs. Several publications were found, reporting the ability of certain organic compounds to form liquid mixtures at room temperature when mixed with $\mathrm{AlCl}_{3}$. In addition, some of these compounds were reported to successfully allow the reversible electrodeposition of aluminium on metallic substrates. ${ }^{8-11}$ Therefore, some of the compounds reported by these papers were tested to verify whether they could work as electrolytes in AIBs. It was quickly found that, for this purpose, the most replicable and promising results were those reported in a publication by $\mathrm{Li}$ et al. ${ }^{11}$ The authors have demonstrated that the mixing acetamide (AcAm) with an excess of $\mathrm{AlCl}_{3}$ gives rise to an ionic liquid that can reversibly electroplate aluminium on a copper substrate. Similarly to what was observed in other $\mathrm{AlCl}_{3}$-organic eutectic mixtures reported in previous works, ${ }^{10,12}$ the electrolyte was reported to likely contain anionic species such as $\mathrm{AlCl}_{4}^{-}$and $\mathrm{Al}_{2} \mathrm{Cl}_{7}^{-}$, and complexed aluminium species such as $\left[\mathrm{AlCl}_{2}(\mathrm{AcAm})\right]^{+}$as cations (Figure 8.1). The aforementioned paper proves that the ionic liquid can effectively electrodeposit aluminium metal under negative potentials, however, no studies had been performed on the possibility of using this eutectic mixture as an electrode in AIBs. Therefore, the ability of the eutectic $\mathrm{AcAm} / \mathrm{AlCl}_{3}$ ionic liquid to effectively substitute the conventional [EMIm]Cl-based electrolytes in AIBs was tested.

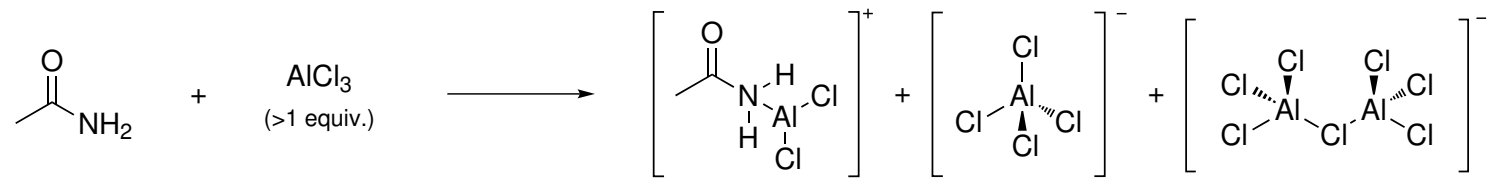

FIGURE 8.1: Diagram illustrating the reaction between acetamide and $\mathrm{AlCl}_{3}$, causing the formation of an ionic liquid. 


\subsubsection{Acetamide: preliminary tests}

A series of ionic liquids were prepared by gradually adding $\mathrm{AlCl}_{3}$ to anhydrous AcAm in a nitrogen-filled glovebox. It could be observed that the addition caused an exothermic reaction, eventually resulting in the formation of a pale-yellow liquid with moderate viscosity. Because the reaction begins in the solid state (AcAm and $\mathrm{AlCl}_{3}$ are solid at room temperature), obtaining a homogeneous reaction occasionally proved to be challenging, as some of the unreacted $\mathrm{AlCl}_{3}$ would get stuck on the walls of the reaction vessel. To counteract this, after the exothermic reaction subsided, the reaction was stirred at $120{ }^{\circ} \mathrm{C}$ until no solid residue could be observed. In order to test the effect of the concentration of $\mathrm{AlCl}_{3}$, three different electrolytes with $\mathrm{AlCl}_{3} / \mathrm{AcAm}$ molar ratios $(r)$ of 1.1, 1.3, and 1.5, were prepared. Higher $r$ values could not be achieved, as 1.5 appeared to be the saturation point and no more $\mathrm{AlCl}_{3}$ could dissolve or react with the electrolyte.

To test their viability as battery electrolytes, the ionic liquids were transferred into a sealed, nitrogen-filled three-electrode electrochemical cell using Schlenk line techniques. For the preliminary tests, a flooded three-electrode setup was chosen to be used, as it is better suited for cyclic voltammetry experiments and gives more reliable voltage values. For all the experiments performed in this setup, two sheets of high-purity aluminium foil were used as the counter and pseudoreference electrode. To ensure that the electroplating mechanism works on an $\mathrm{Al}$ electrode the same way it was demonstrated by $\mathrm{Li}$ et al. ${ }^{11}$ on a $\mathrm{Cu}$ substrate, cyclic voltammetry experiments were first performed using aluminium foil as the working electrode (Figure 8.2).

The voltammograms show a behaviour that is very similar to what was previously reported for the reversible electroplating of aluminium from [EMIm] Cl/ $\mathrm{AlCl}_{3}$ mixtures ${ }^{1}$ or other organic-inorganic electrolytes. ${ }^{8,13}$ First, as the voltage decreases below $0 \mathrm{~V}$, an increasingly negative current develops, imputable to the bulk reduction of $\mathrm{Al}_{2} \mathrm{Cl}_{7}^{-}$and deposition of $\mathrm{Al}$ metal onto the electrode. Then, a positive current was observed, with a plateau at about $0.85 \mathrm{~V}$, corresponding to the stripping of the deposited $\mathrm{Al}$ metal. The equation for the underlying electrochemical process is assumed to be the following:

$$
4 \mathrm{Al}_{2} \mathrm{Cl}_{7}^{-}+3 \mathrm{e}^{-} \rightleftharpoons 7 \mathrm{AlCl}_{4}^{-}+\mathrm{Al}
$$




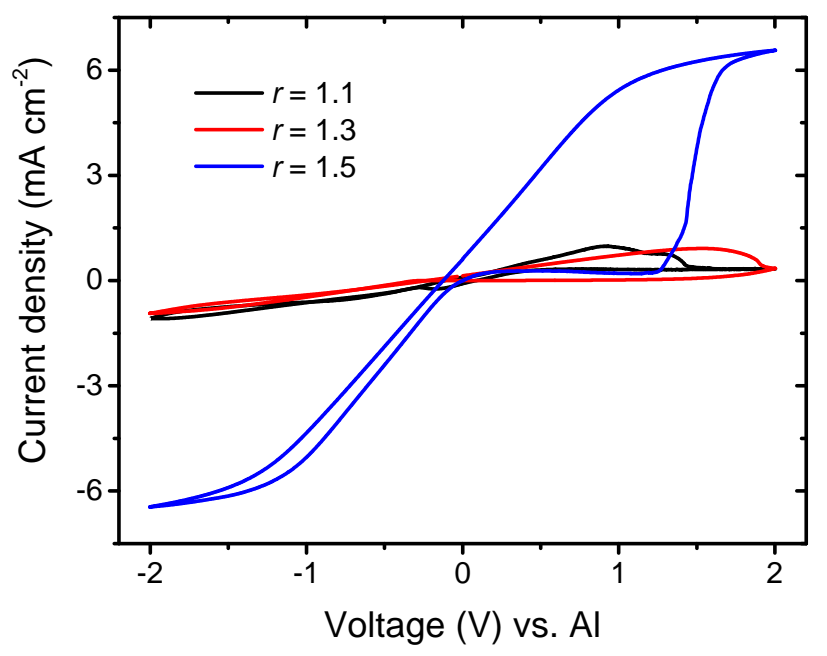

FIGURE 8.2: Cyclic voltammograms (third cycle, using a flooded three-electrode cell) of $\mathrm{AcAm} / \mathrm{AlCl}_{3}$ electrolytes with different $r$ values, using $\mathrm{Al}$ foil as the working electrode, showing the electroplating of $\mathrm{Al}$ metal. The current density is referred to the projected area of the working electrode; the voltage is measured against an Al foil pseudo-reference electrode. Adapted by permission of The Royal Society of Chemistry. ${ }^{7}$

It is worth noting that, as the $r$ value of the electrolyte increases, the concentration of $\mathrm{Al}_{2} \mathrm{Cl}_{7}^{-}$ions must also increase, as a result of the added $\mathrm{AlCl}_{3}$ forming a complex with the existing $\mathrm{AlCl}_{4}^{-}$ions: ${ }^{14}$

$$
\mathrm{AlCl}_{3}+\mathrm{AlCl}_{4}^{-} \rightleftharpoons 4 \mathrm{Al}_{2} \mathrm{Cl}_{7}^{-}
$$

This mechanism can therefore explain why the electrolyte with $r=1.5$ shows much a much higher current density than the other electrolytes: its higher concentration of $\mathrm{Al}_{2} \mathrm{Cl}_{7}^{-}$ions causes a higher electrodeposition rate, resulting in higher faradaic currents at the electrode.

Previous sections of this thesis described how graphitic and graphene-based materials are perhaps the most promising cathode materials for AIBs (see also Sections 2.4.2 and 5.1). Because of this, the behaviour of our electrolytes in the presence of a graphitic material was also investigated. Pyrolytic graphite paper in particular has been previously established as a good benchmark material for electrolyte performance testing thanks to its availability, low cost and ease of use. $., 6,15$ Therefore, the ability of the $\mathrm{AcAm} / \mathrm{AlCl}_{3}$ electrolytes to allow intercalation of 


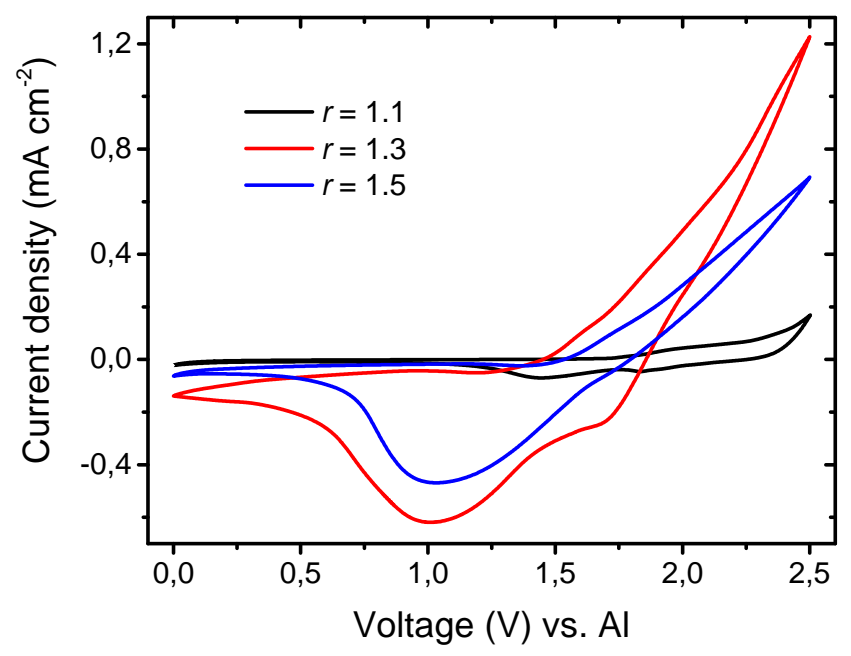

FIGURE 8.3: Cyclic voltammograms (third cycle, using a flooded three-electrode cell) of AcAm $/ \mathrm{AlCl}_{3}$ electrolytes with different $r$ values, using pyrolytic graphite paper as the working electrode, showing the intercalation of $\mathrm{AlCl}_{4}^{-}$ions. The current density is referred to the projected area of the working electrode; the voltage is measured against an Al foil pseudo-reference electrode. Adapted by permission of The Royal Society of Chemistry. ${ }^{7}$

$\mathrm{AlCl}_{4}^{-}$ions into a graphitic carbon cathode was then probed by performing a series of cyclic voltammetry experiments using pyrolytic graphite paper as the working electrode (Figure 8.3).

The plots show an easily recognisable pattern, ${ }^{6,16,17}$ which can be interpreted in the following way: the increase in current above $1.4 \mathrm{~V}$ can be ascribed to the insertion of $\mathrm{AlCl}_{4}^{-}$ions into the graphitic lattice, and the reduction peaks in the 0.6-2 $\mathrm{V}$ range correspond to the consecutive deintercalation of the chloroaluminate ions. This is in good agreement with the staging intercalation-deintercalation mechanism that has been proposed and demonstrated for graphitic and graphene cathodes in previous works, ${ }^{2,17-19}$ according to the following equation:

$$
\mathrm{xC}+\mathrm{AlCl}_{4}^{-} \rightleftharpoons \mathrm{C}_{\mathrm{x}}\left(\mathrm{AlCl}_{4}\right)+\mathrm{e}^{-}
$$

It can be seen from the plots that increasing the $r$ value from 1.1 to 1.3 leads to a sharp increase in both the cathodic and anodic peaks in the voltammogram, as well as a shift to lower potentials. This trend can be attributed to the very notable difference in viscosity between the two electrolytes, as the electrolyte with $r=1.3$ 
is visibly less viscous. This translates into increased ion mobility, which helps in reducing the overpotential barrier for the intercalation and deintercalation reactions and increasing the reaction rate, leading to a higher current intensity. This increase in current is partially reversed when further increasing $r$ to 1.5. It is not clear why this trend inversion happens, but a possible explanation might be due to the change in the anionic composition of the ionic liquid: as more and more $\mathrm{AlCl}_{3}$ gets dissolved, more $\mathrm{AlCl}_{4}^{-}$ions are complexed to form $\mathrm{Al}_{2} \mathrm{Cl}_{7}^{-}$ions. As described by Equation 8.3, $\mathrm{AlCl}_{4}^{-}$ions are the ones partaking in the cathode intercalation reaction. Therefore, as their availability decreases, the rate of intercalation and deintercalation reactions will likely also decrease, leading to a lower faradaic current.

A series of galvanostatic charge-discharge experiments were then performed on the electrolytes, using the previously described cell design, to test their behaviour in a more battery-specific process. In order to compare our results with other tests performed in the literature, the experiments were performed within the voltage window of $0.3-2.45 \mathrm{~V}$, using a relatively slow cycling rate of $83 \mathrm{~mA} \mathrm{~g}^{-1}$. The results of such tests are shown in Figure 8.4. It is worth noting that, even in this case,

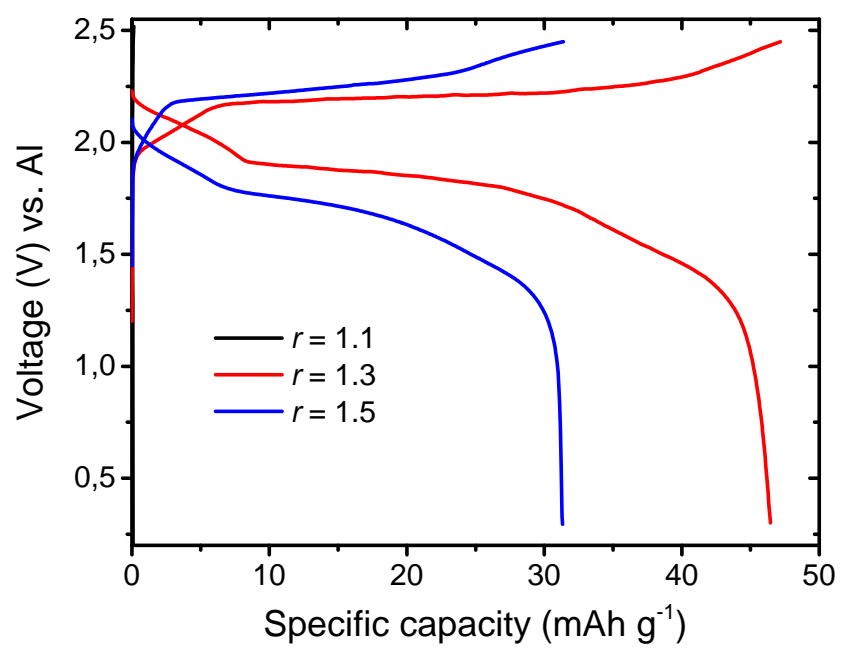

FIGURE 8.4: Typical galvanostatic charge-discharge cycles (tenth cycle, using a flooded three-electrode cell) of $\mathrm{AcAm} / \mathrm{AlCl}_{3}$ electrolytes with different $r$ values, using pyrolytic graphite paper as the working electrode. The voltage is measured against an Al foil pseudo-reference electrode. Adapted by permission of The Royal Society of Chemistry. ${ }^{7}$ 
the first cycle of such tests is always characterised by a significantly longer charging phase (see also Figure A.18), corresponding to a low coulombic efficiency. This phenomenon has been reported previously for pyrolytic graphite paper, ${ }_{1}^{15,20,21}$ and evidence suggests that it is caused by the formation of a solid-electrolyte interphase (see also Section 5.2.2). The data shows well-defined voltage plateaus for the charge and discharge profiles, which are very similar to the ones reported for $[\mathrm{EMIm}] \mathrm{Cl} / \mathrm{AlCl}_{3}$-based electrolytes in previous publications. ${ }^{2,15}$ The same explanation described previously for the cyclic voltammetry results could also justify the trend obtained for the galvanostatic charge-discharge profiles: electrolytes with $r=1.3$ performed slightly better than the electrolyte with $r=1.5$, as demonstrated by the narrower voltage gap between the charge and discharge plateau, and the higher specific capacity achieved. This is possibly due to the higher concentration of the $\mathrm{AlCl}_{4}^{-}$species participating in the intercalation reaction. Both electrolytes perform significantly better than the one with $r=1.1$, which achieved a negligible discharge capacity of less than $1 \mathrm{mAh} \mathrm{g}^{-1}$. This is again likely due to the high viscosity of the liquid, corresponding to low ion mobilities, which could cause an increase in the internal resistance in the device, and a very slow rate of intercalation of $\mathrm{AlCl}_{4}^{-}$ions. The maximum discharge capacity value obtained for the $\mathrm{AcAm} / \mathrm{AlCl}_{3}$ electrolytes is $46 \mathrm{mAh} \mathrm{g}{ }^{-1}$, which was achieved by the $r=1.3 \mathrm{sam}-$ ple. This value is notably lower than previously reported by existing publications for pyrolytic graphite paper, ${ }^{15,16}$ which is also likely due to the higher viscosity of our new electrolytes compared to the conventional [EMIm] $\mathrm{Cl} / \mathrm{AlCl}_{3}$-based systems. On the other hand, the $\mathrm{AcAm} / \mathrm{AlCl}_{3}$ electrolytes show very high coulombic efficiencies, indicating the absence of unwanted reactions in the system.

\subsubsection{Diluted electrolytes}

The previous experiments highlighted an interesting observation: reducing the viscosity of the electrolytes is the key to achieve an improvement in the performance of the devices. Because of this, the possibility of diluting the electrolytes with an appropriate solvent was investigated. Due to the high polarity and reactivity of the electrolytes, finding a suitable solvent to use as dilutant was not a trivial task: several solvents with various chemical structures and degrees of polarity were tested, such as acetonitrile, chloroform, NMP, DMF, toluene, propylene 
carbonate, tetrahydrofuran, and dimethyl sulfoxide. Ultimately, dichloromethane (DCM) seemed to perform best by having great miscibility with the electrolyte and no apparent reactivity. The electrolytes were therefore diluted with increasing volume fractions of DCM, and the effects of the dilution were tested by conducing more galvanostatic charge-discharge tests, using the same conditions described above.

It can be seen from Figure 8.5 (left) that even the addition of only a 10\% volume fraction of DCM to the electrolyte can greatly enhance the discharge capacity from 46 to $67 \mathrm{mAh} \mathrm{g}^{-1}$. Further dilution to a $30 \%$ volume fraction of DCM can increase this value up to $77 \mathrm{mAh} \mathrm{g}^{-1}$, which is consistent with what other authors have found to be the maximum capacity for pyrolytic graphite paper. ${ }^{15,16}$ The voltage gap between the charge and discharge plateaus is also reduced significantly, indicating lower internal resistance. The profiles also show better defined sections where the different stages of intercalation and deintercalation take place, ${ }^{22}$ indicating an overall improvement in the performance of the device. Similar enhancements can also be observed in cyclic voltammetry data (Figure A.19). The coulombic efficiency of the process, however, tends to decrease with the increase of the dilution fraction. This is due to DCM taking part in side reactions during
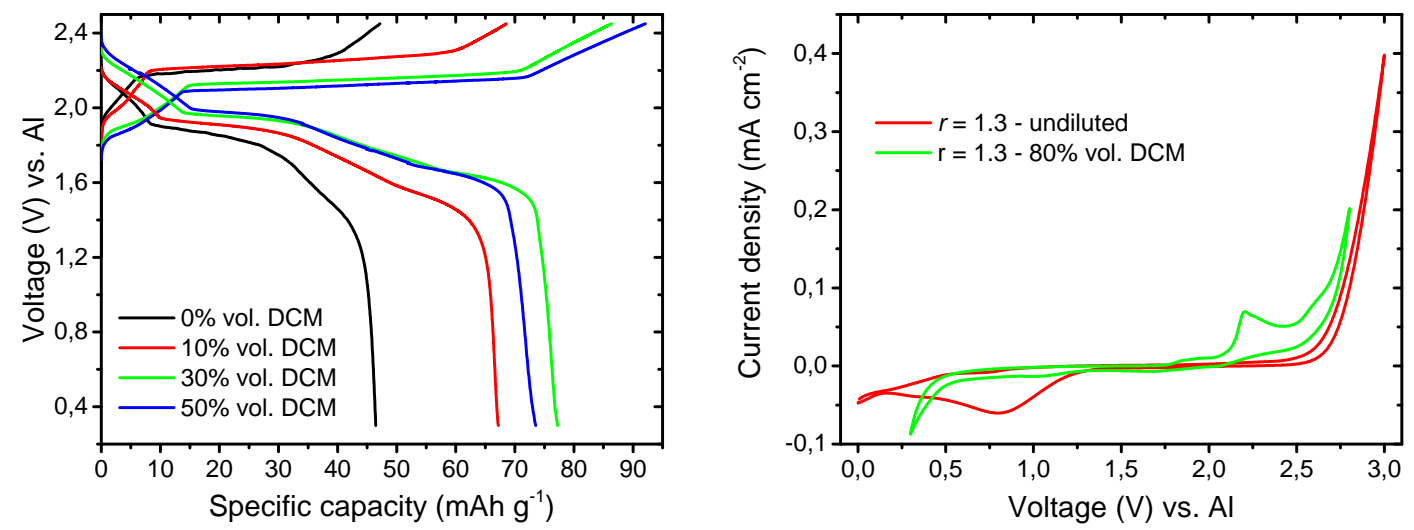

FIGURE 8.5: (Left) Typical galvanostatic charge-discharge cycles (tenth cycle, using flooded three-electrode cells) of AcAm/ $\mathrm{AlCl}_{3}$ electrolytes, with $r=1.3$, diluted with increasing quantities of DCM. (Right) Cyclic voltammograms of pure and DCM-diluted AcAm/ $\mathrm{AlCl}_{3}$ electrolytes in a three-electrode cell, using a glassy carbon working electrode. The current density is referred to the projected cathode area; voltages measured against an $\mathrm{Al}$ foil pseudo-reference electrode.

Adapted by permission of The Royal Society of Chemistry. ${ }^{7}$ 
the charge phase.

This hypothesis was confirmed by performing cyclic voltammetries of the electrolytes using a glassy carbon working electrode: unlike graphite, the material is mostly inert in the electrolyte environment and does not allow intercalation of $\mathrm{AlCl}_{4}^{-}$. Therefore, the stability window of the electrolyte should be precisely assessed by using this electrode, as the only faradaic currents detectable should be imputable to the decomposition reactions of the electrolyte. It can be seen from Figure 8.5 (right) that an irreversible oxidation peak around $2.2 \mathrm{~V}$ is present in the $\mathrm{CV}$ for the diluted electrolyte. Furthermore, the onset of bulk electrolyte decomposition is found at lower voltages compared to the undiluted electrolyte. Overall, the trends described in this section and the conclusions derived from the dilution data above also holds true for the electrolytes with $r=1.5$, as demonstrated briefly in Figure 8.6.

Based on the data displayed thus far, it would appear that adding between 10 and $30 \%$ volume of DCM to the electrolytes would be the optimal value to increase the specific capacity of the device, without excessively sacrificing its coulombic efficiency.
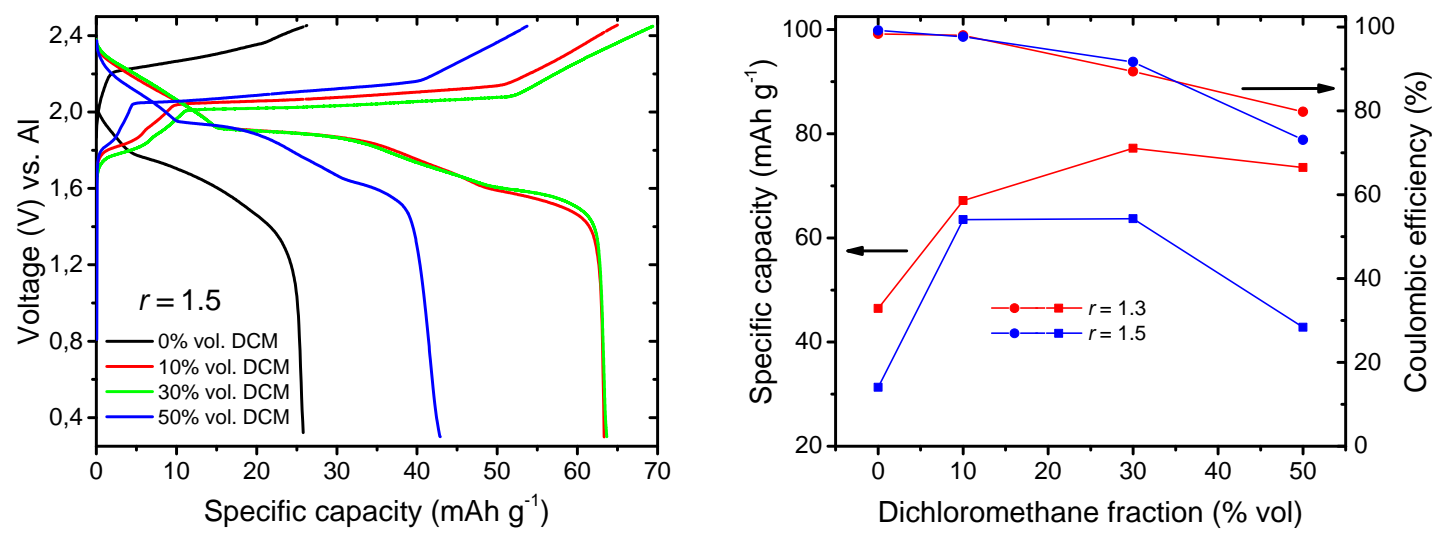

FIGURE 8.6: (Left) Typical galvanostatic charge-discharge profiles (tenth cycle, using flooded three-electrode cells) of AcAm $/ \mathrm{AlCl}_{3}$ electrolytes, with $r=1.5$, diluted with increasing quantities of DCM. The voltage is measured against an Al foil pseudo-reference electrode. (Right) Specific discharge capacities (square) and coulombic efficiencies (dot) of $\mathrm{AcAm} / \mathrm{AlCl}_{3}$ ionic liquids with different $r$ values, diluted with increasing quantities of DCM. Adapted by permission of The Royal Society of Chemistry. ${ }^{7}$ 


\subsubsection{Investigating "ultrafast" cycling rates}

As previously discussed (Section 5.1) one of the most attractive features of AIBs is their ability to withstand "ultrafast" charging and discharging capabilities without losing any significant specific capacity. ${ }^{16,23}$ It is therefore very important to ensure that any alternative electrolyte developed for AIBs still allows such ultrafast rate features without impacting device performance negatively. To test this, more galvanostatic cycling tests were run using different current rates on devices implementing the electrolytes, and compared the results with the performance of the standard [EMIm]Cl-based electrolyte. The results are reported in Figure 8.7.

The graph shows that at the relatively slow rate of $83 \mathrm{~mA} \mathrm{~g}^{-1}$, the discharging specific capacity of the AcAm-based electrolytes is comparable with the literature standard. If the the cycling current is raised to $208 \mathrm{~mA} \mathrm{~g}^{-1}$, a predictable loss of specific capacity takes places for all the electrolytes tested, with the $30 \%$ diluted $\mathrm{AcAm} / \mathrm{AlCl}_{3}$ electrolyte still performing very close to the literature standard. Further increasing the current to $416 \mathrm{~mA} \mathrm{~g}^{-1}$ leads to the performance gap between the novel electrolytes and the literature standard to widen considerably. At this rate, the $50 \%$ diluted electrolyte is the one closest to the literature standard electrolyte, yielding a specific discharge capacity of about $20 \mathrm{mAh} \mathrm{g}^{-1}$, compared to a capacity of about $30 \mathrm{mAh} \mathrm{g}^{-1}$ obtained by the [EMIm]Cl electrolyte. At the fastest

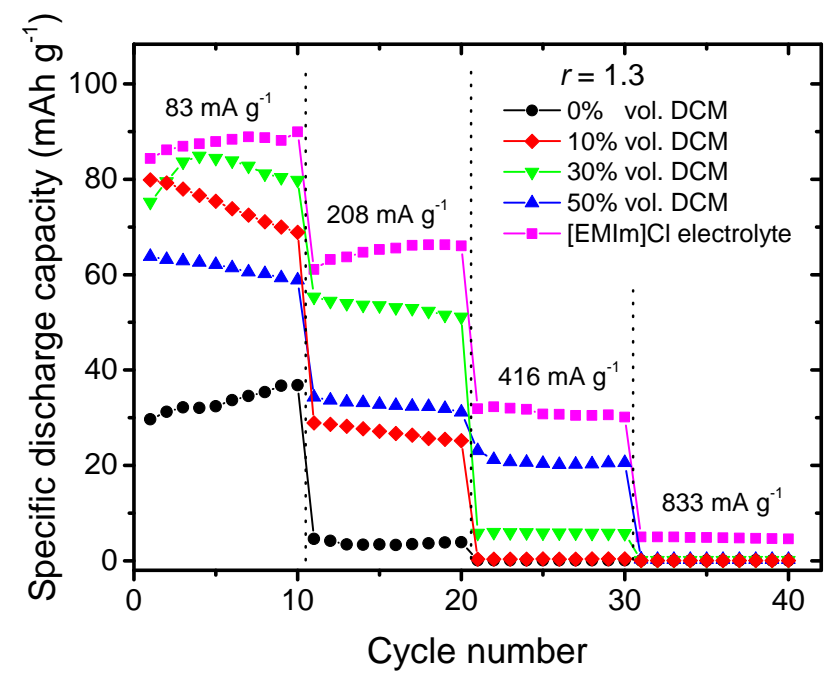

FIGURE 8.7: Galvanostatic cycling (using flooded three-electrode cells) at different currents of the $\mathrm{AcAm} / \mathrm{AlCl}_{3}$ electrolytes, compared with the [EMIm] $\mathrm{Cl}$ electrolyte. Adapted by permission of The Royal Society of Chemistry. ${ }^{7}$ 
charging rate tested of $833 \mathrm{~mA} \mathrm{~g}^{-1}$, no electrolyte could provide a discharge capacity above $10 \mathrm{mAh} \mathrm{g}^{-1}$. Although the use of the AcAm/DCM-based electrolytes appears to cause a loss in specific discharge capacity at higher current rates, it is worth noting that even the devices built using the [EMIm]Cl-based electrolytes, which is what had been used for ultrafast devices in previous works, ${ }^{16}$ suffer a consistent loss in capacity when the charging/discharging rate is increased to higher currents. This information suggests that most of the "ultrafast" features of AIBs are imputable to the nanostructure of the "graphitic foam"-type cathodes used in previous publications, and the electrolyte has little impact on the rate capabilities of the device. Therefore, there is no evidence that the AcAm-based electrolytes would hinder the performance of an ultrafast AIB.

\subsubsection{Swagelok-type cell tests}

After seeing the promising results of the $\mathrm{AcAm} / \mathrm{AlCl}_{3}$ electrolytes in a threeelectrode cell setup, their performance was also tested in a Swagelok-type cell. The devices were assembled using a pyrolytic graphite disc as the cathode and $\mathrm{Al}$ foil as the anode. First, two devices were built using the undiluted $r=1.3$ and $r=$ 1.5 electrolytes. Then, galvanostatic cycling was performed on the devices in the same voltage range as previous experiments using a rate of $50 \mathrm{~mA} \mathrm{~g}^{-1}$. Typical profiles for the charging and discharging profiles are shown in Figure 8.8.

The charging and discharging profiles appear somewhat similar to the ones obtained from the tests performed on the three-electrode setup except for a few key differences:

- The charging and discharging plateaus are less defined and fall in a broader voltage range.

- The "kinks" in the curves, related to the different stages of graphite intercalation, are less defined.

- The electrolyte with $r=1.5$ yields a higher specific capacity than its $r=1.3$ counterpart.

Such behaviour could be explained by the differences in cell design compared to the previous experiments. First of all, the use of a two-electrode configuration for the electrochemical cell (i.e. using the anode as both counter and reference electrode) means that the voltage values are not as reliable, leading to a loss in 


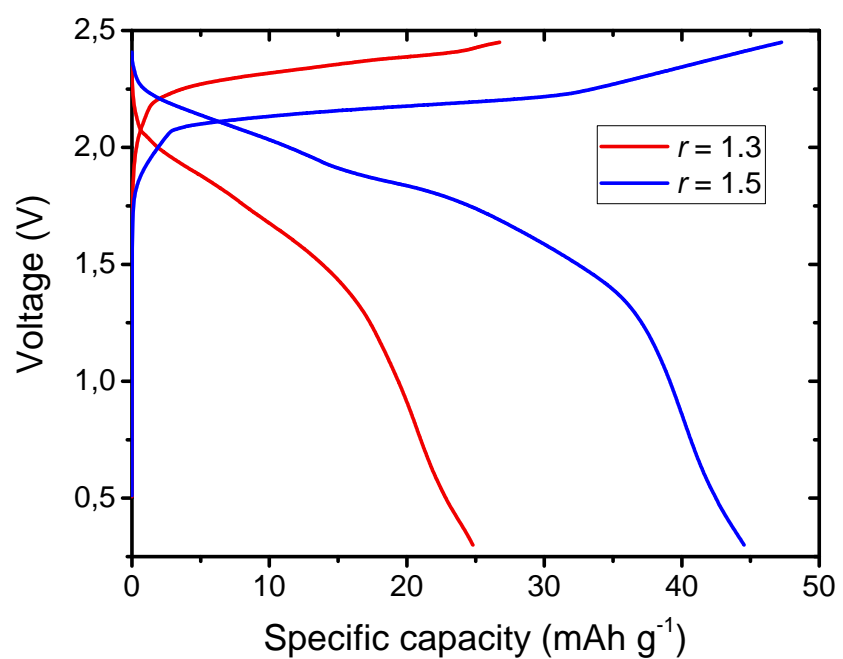

FIGURE 8.8: Typical galvanostatic charge-discharge profile (tenth cycle) of Swagelok-type cells built using $\mathrm{AcAm} / \mathrm{AlCl}_{3}$ electrolytes, with different $r$ ratios.

The voltage reported in this plot is the cell potential.

the accuracy of the measurements. Furthermore, factors such as the ohmic drop, limiting currents, and concentration overpotentials are heavily influenced by the geometry of the electrochemical cell. In particular, as the reactive species in the electrolyte are consumed or supplied at both electrodes, there is going to be a transition potential in which the concentration of reactive species reaches zero or saturation: this transition depends on cell geometry, as well as the electrolyte composition and viscosity. ${ }^{24}$ Regardless of the individual factors contributing to these changes, the data suggests that the reason for the $r=1.5$ electrolyte being the best performer in the case of Swagelok-type cells is that in this cell architecture, electrolyte viscosity becomes the predominant driving force in the performance of the device. This would therefore prevail over the concentration of $\mathrm{AlCl}_{4}^{-}$ions, which constituted the limiting factor in a flooded 3-electrode cell setup. An alternative explanation is that, when using the Swagelok-type cell setup, the electrodeposition of aluminium at the anode (Equation 8.1) becomes the "rate-determining" process in the cell; therefore, the higher concentration of $\mathrm{Al}_{2} \mathrm{Cl}_{7}^{-}$in the $r=1.5$ electrolyte would lead to a better overall performance in the device. At this stage, it is unclear what the actual reason for this trend inversion is; more in-depth tests such, as Tafel analysis to determine the rate-determining step, or electrochemical impedance spectroscopy to discern individual cell components contributing to its 
internal resistance, could help elucidate its causes.

Galvanostatic cycling experiments were then performed on the diluted electrolytes. Figure 8.9 shows a typical profile for a device built using the $r=1.3$ AcAm $/ \mathrm{AlCl}_{3}$ electrolyte, diluted with a 30\% volume fraction of DCM, which was among the best-performing electrolytes in the previously used cell setup. It can be seen from the plot that although the galvanostatic profiles look more defined than the ones relative to the undiluted electrolyte, the other positive effects given by dilution of the electrolyte in a three-electrode setup appear to be completely negated when a prototype battery device is used, as the specific discharge capacity is comparable to the one relative to the undiluted electrolyte. Furthermore, the specific charging capacity is much higher than discharge capacity, corresponding to a very low coulombic efficiency of about $40 \%$. This loss of coulombic efficiency is present in all the $r$ ratios of electrolytes, and is more dramatic with increasing volumes of DCM added. It is also worth noting that, unlike what was observed for undiluted electrolytes, such efficiency loss is persistent throughout all the galvanostatic cycles run on the device and does not subside after the first cycle (Figure A.20). Therefore, this effect is unlikely to be related to the formation of SEI described in Section 5.2.2, but is instead imputable to the side-reactions and decomposition of

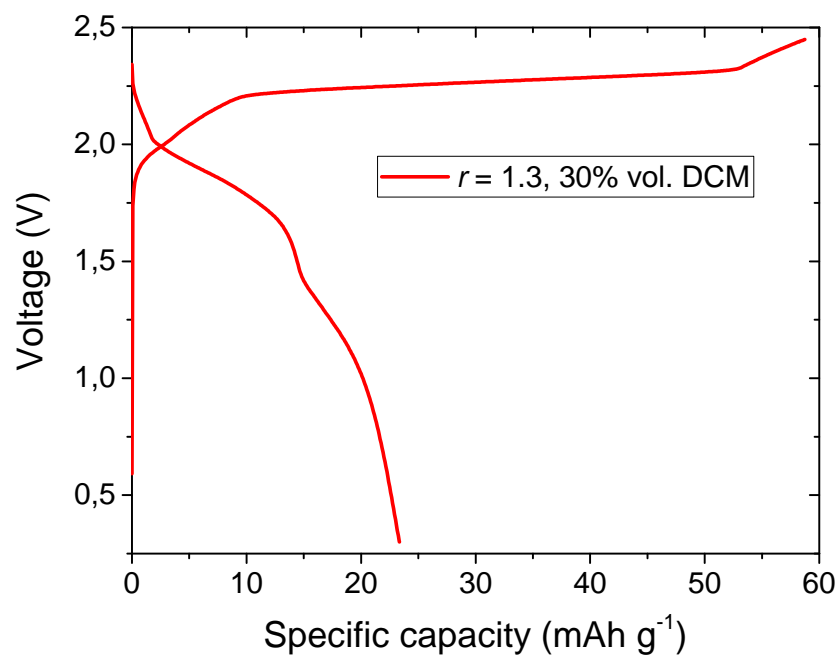

FIGURE 8.9: Typical galvanostatic charge-discharge profile (tenth cycle) of a Swagelok-type cell built using the $r=1.3 \mathrm{AcAm} / \mathrm{AlCl}_{3}$ electrolytes, diluted with a $30 \%$ volume fraction of DCM. The voltage reported in this plot is the cell potential. 
dichloromethane. This result could also be an effect of the different cell design, which could favour such undesirable side reactions.

The results of the cycling tests indicate that dilution with dichloromethane is not a suitable strategy for increasing device performance, and therefore, a different strategy needs to be adopted.

\subsection{Other amide/ $\mathrm{AlCl}_{3}$ eutectic mixtures}

\subsubsection{Alternative amides as electrolytes}

Because of the poor performance of DCM-diluted electrolytes in Swagelok-type cells, a different methodology needed to be investigated to improve the performance of our novel electrolytes. Looking into the literature, it appears that other amide compounds can form eutectic mixtures with $\mathrm{AlCl}_{3} .5,25-27$ It was therefore decided to test whether other analogue compounds could work as battery electrolyte, and perhaps yield better performance.

A number of simple amides were initially surveyed by testing their ability to form room temperature liquids when mixed with an excess of $\mathrm{AlCl}_{3}$. The compounds examined are $\mathrm{N}, \mathrm{N}^{\prime}$-dimethylacetamide (DMAc), N-methylacetamide (NMAc), DMF, and propionamide (PrAm) (Figure 8.10). These compounds were selected because of their low cost, simple molecular structure, and similarity with acetamide.
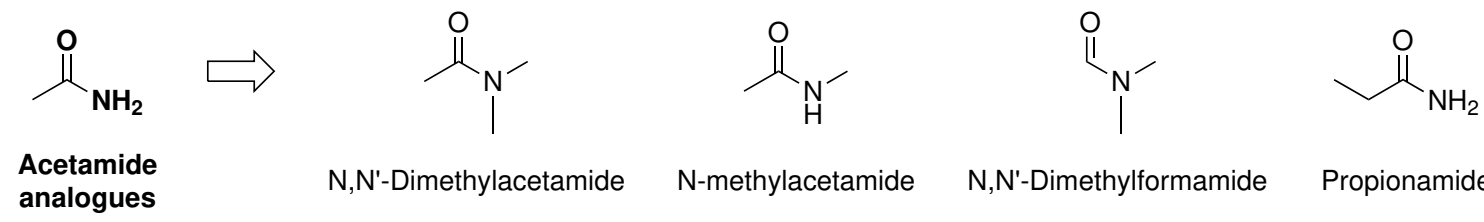

N,N'-Dimethylacetamide

$\mathrm{N}$-methylacetamide

$\mathrm{N}, \mathrm{N}$-'Dimethylformamide

Propionamide

FIGURE 8.10: Diagram showing the molecular structure of the acetamide analogues tested to be used as electrolytes.

In order to verify their ability to form eutectic mixtures, 1.3 equivalents of $\mathrm{AlCl}_{3}$ were mixed to to 1 equivalent of each amide under magnetic stirring and, if necessary, heated to $120^{\circ} \mathrm{C}$ to complete dissolution. Of the compounds tested, PrAm and NMAc could form a room temperature ionic liquid with $\mathrm{AlCl}_{3}$, and DMF and DMAc produced a solid. This was somewhat expected in the case of DMF, as the compound manifested a similar behaviour when used in small quantities as a 


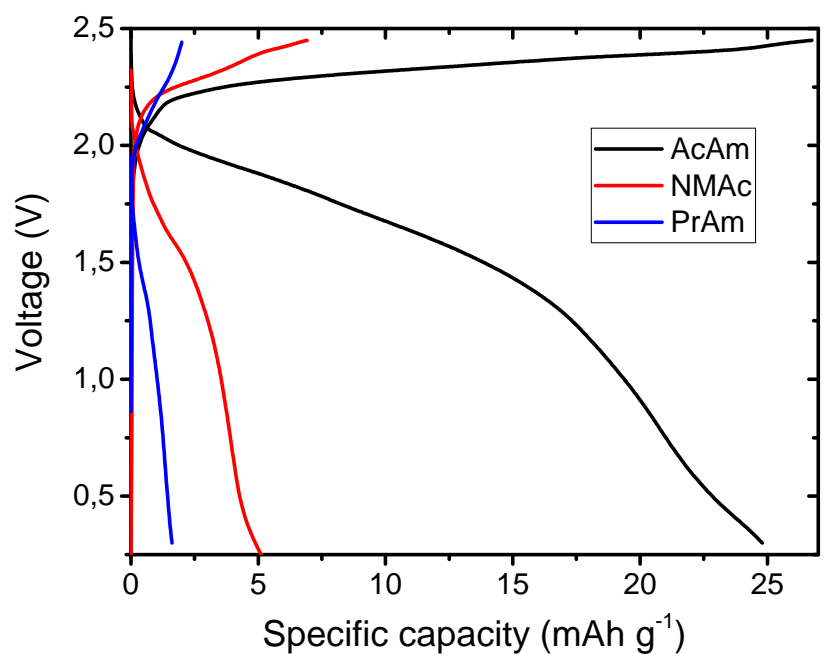

FIGURE 8.11: Typical galvanostatic charge-discharge profiles (tenth cycle, using a current rate of $50 \mathrm{~mA} \mathrm{~g}^{-1}$ ) of Swagelok-type cells built using alternative amidebased electrolytes, with an $r$ ratio of 1.3 , compared to the $\mathrm{AcAm} / \mathrm{AlCl}_{3}$ electrolyte.

The voltage reported in this plot is the cell potential.

diluent to the AcAm/ $\mathrm{AlCl}_{3}$ electrolytes. DMAc, on the other hand, formed a liquid at high temperatures $\left(>80^{\circ} \mathrm{C}\right)$, but was solid at room temperature. Although NMAc and PrAm could successfully yield room-temperature liquids, their viscosity appeared higher than the AcAm-based electrolytes.

The cycling performance of the newly obtained NMAc- and PrAm-based electrolytes was therefore compared to their $\mathrm{AcAm} / \mathrm{AlCl}_{3}$ counterpart by assembling devices using these electrolytes and performing a series of galvanostatic cycling tests using a current rate of $50 \mathrm{~mA} \mathrm{~g}^{-1}$ (Figure 8.11). The graph shows that although the new electrolytes can effectively yield a somewhat functional chargedischarge profile, their performance is rather poor, as NMAc and PrAm could only achieve a discharge capacity of 5 and $2 \mathrm{mAh} \mathrm{g}^{-1}$ for their tenth cycle, respectively, compared to the much larger capacity of about $25 \mathrm{mAh} \mathrm{g}{ }^{-1}$ of the $\mathrm{AcAm} / \mathrm{AlCl}_{3}$ electrolyte. This result is aligned with the higher viscosity we observed in the newly prepared samples. Therefore, although NMAc and PrAm were proven to work similarly to acetamide in the formation of electrolytes for AIBs, their performance is quite disappointing. 


\subsubsection{Mixed amide electrolytes}

In light of the previously described experiments, it was hypothesised that the high viscosity in the electrolytes, responsible for the underwhelming performance of the devices, is mainly owed to the formation of strong ionic pairs between the chloroaluminate ions and the $\mathrm{AlCl}_{2}$-amide cations. Generally speaking, the viscosity of a fluid can be reduced by decreasing the strength of the intermolecular interactions taking place between its components. In an ionic liquid, the most predominant intermolecular interaction is likely the ionic pair interaction, in which particles with opposite charge tend to lie in close proximity with each other, forming dimeric structures, or in more extreme cases, even clusters of molecules. ${ }^{28,29} \mathrm{It}$ was previously observed that dilution of the electrolytes with solvents is a valid method to enhance the performance of the electrolyte by reducing its viscosity, as the addition of solvent molecules to the mixtures would likely disrupt the strong ionic pair interactions present in the liquid. Such method however proved to be quite tricky, due side reactions taking place either during the addition of the diluting compounds to the ionic liquids (i.e. the diluting compound reacting with the species in the ionic liquid), or during the electrochemical processes (i.e. the diluting compound decomposing in the voltage range used for battery operation).

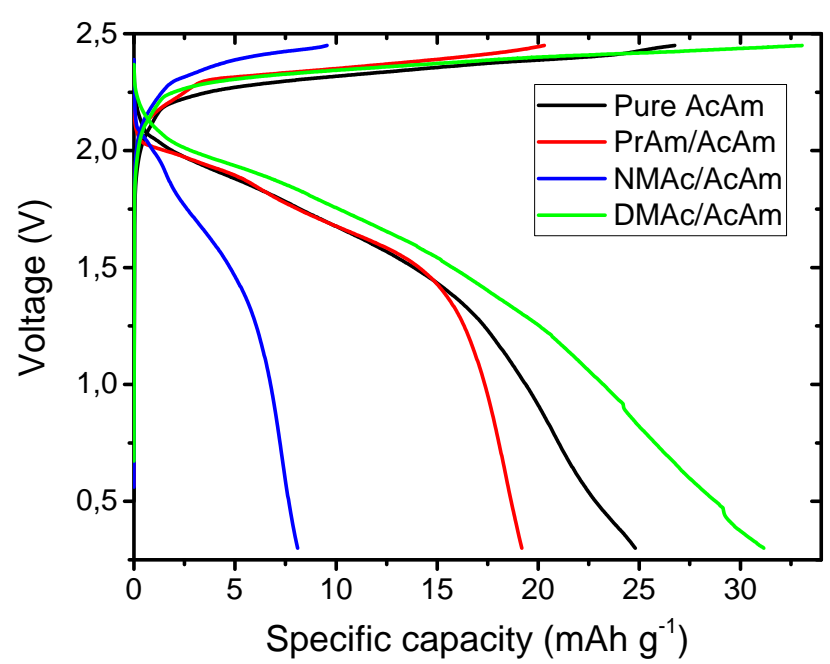

FIGURE 8.12: Typical galvanostatic charge-discharge profiles (tenth cycle, using a current rate of $50 \mathrm{~mA} \mathrm{~g}^{-1}$ ) of Swagelok-type cells built using mixed amide-based electrolytes, with an $r$ ratio of 1.3, compared to the AcAm/ $\mathrm{AlCl}_{3}$ electrolyte. The voltage reported in this plot is the cell potential. 
An alternative method to reduce the viscosity could consist of using mixtures of amides in the preparation of the electrolytes. This way, the resulting heterogeneity of the ionic species would add a degree of disorder in the mixture, possibly weakening the ionic pair interactions in the system, and effectively lowering the viscosity of the electrolyte. Furthermore, by using amides known to work individually, the chance of unwanted side reactions should be reasonably minimised. This strategy was initially tested by adding 1.3 molar equivalents of $\mathrm{AlCl}_{3}$ to mixtures made of 0.5 equivalents of AcAm and 0.5 equivalents of another amide. When using these ratios, all the tested mixtures, except for the one using DMF as the second amide, could effectively form room temperature ionic liquids. Devices were then assembled using these electrolytes and galvanostatic cycling tests were performed on the devices, using the same conditions as previous experiments (Figure 8.12). It can be observed from the graph that the mixed electrolytes achieved different levels of performance. Specifically, the NMAc/AcAm mixture performed notably worse than pure AcAm while the PrAm/AcAm mixture performed comparably to the pure AcAm-based electrolyte and the DMAc/AcAm mixture achieved slightly higher discharge capacity values. This result somewhat confirms the initial hypothesis that adding disorder at the molecular level to the system by using a mixture of amides can result in an improvement in the performance. This is likely due to the fact that by using amide pairs instead of single amides the intermolecular interactions in the liquid are weakened, resulting in a reduction of its viscosity.

In order to further test this hypothesis, an additional electrolyte using PrAm and AcAm in 70/30 a molar ratio was prepared while maintaining $r$ at 1.3. The comparison between the galvanostatic cycling data of this electrolyte and the one relative to the other PrAm- and AcAm-containing mixtures is displayed in Figure 8.13. It can be seen how the $70 / 30$ ratio electrolyte vastly outperforms both its pure counterparts and the 50/50 ratio, achieving a specific discharge capacity of over $40 \mathrm{mAh} \mathrm{g}^{-1}$. This is an interesting result, as it indicates the presence of a "sweet spot" in the PrAm/AcAm ratio in which the performance of the device is maximised. This could be due to the composition of the electrolyte reaching an optimal state in which the ionic mobility is maximised. Further studies would need to be performed to shed light on this phenomenon.

Finally, a selection of the best-performing mixed amide electrolytes was prepared 


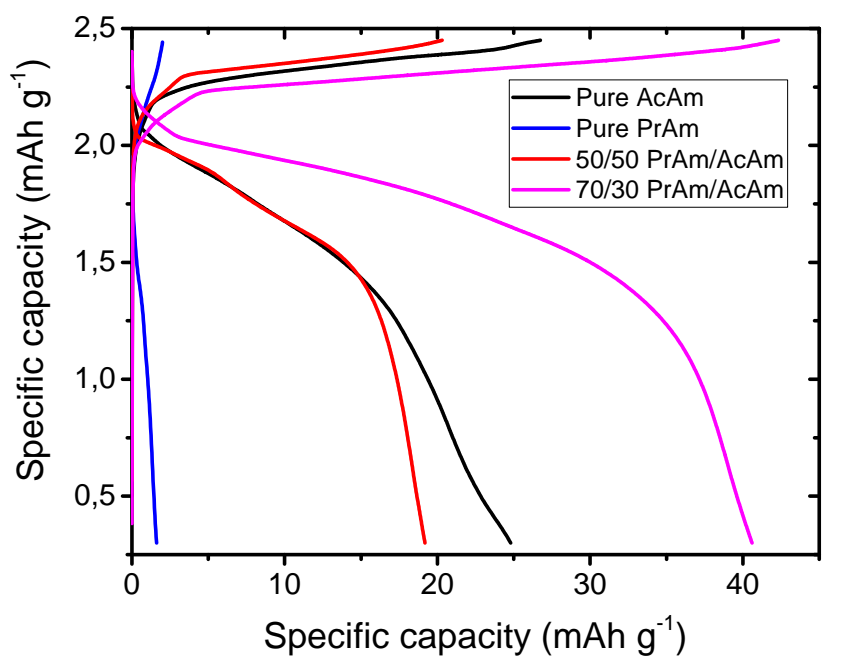

FIGURE 8.13: Typical galvanostatic charge-discharge profiles (tenth cycle, using a current rate of $50 \mathrm{~mA} \mathrm{~g}^{-1}$ ) of Swagelok-type cells built using mixed PrAm/AcAm electrolytes, with an $r$ ratio of 1.3. The voltage reported in this plot is the cell potential.

again using a $1.5 r$ ratio, to verify whether the higher $\mathrm{AlCl}_{3}$ content improves the performance of those electrolytes as well. The results of the galvanostatic cycling tests performed using such electrolytes are presented in Figure 8.14. The graph shows that the $r=1.5$ electrolytes perform notably better than their $r=1.3$ counterparts: the higher molar ratio of $\mathrm{AlCl}_{3}$ increases the discharge capacity of the 50/50 DMAc/AcAm electrolyte from 31 to $45 \mathrm{mAh} \mathrm{g}^{-1}$, and that of the 70/30 PrAm/AcAm electrolyte from 41 to almost $60 \mathrm{mAh} \mathrm{g}^{-1}$, corresponding to a notable improvement of about $50 \%$ in both cases. In can be also seen from the graph that the performance of the best-performing electrolyte is almost approaching the one of the state-of-the-art [EMIm]Cl-based electrolyte. Furthermore, the 70/30 PrAm/AcAm electrolyte electrolyte also shows good stability and cycling life in extended charge-discharge tests, as demonstrated in Figure A.21. This is a very exciting result, as it indicates that amides could definitely constitute a viable lowcost alternative to expensive organic salts such as [EMIm] Cl. 


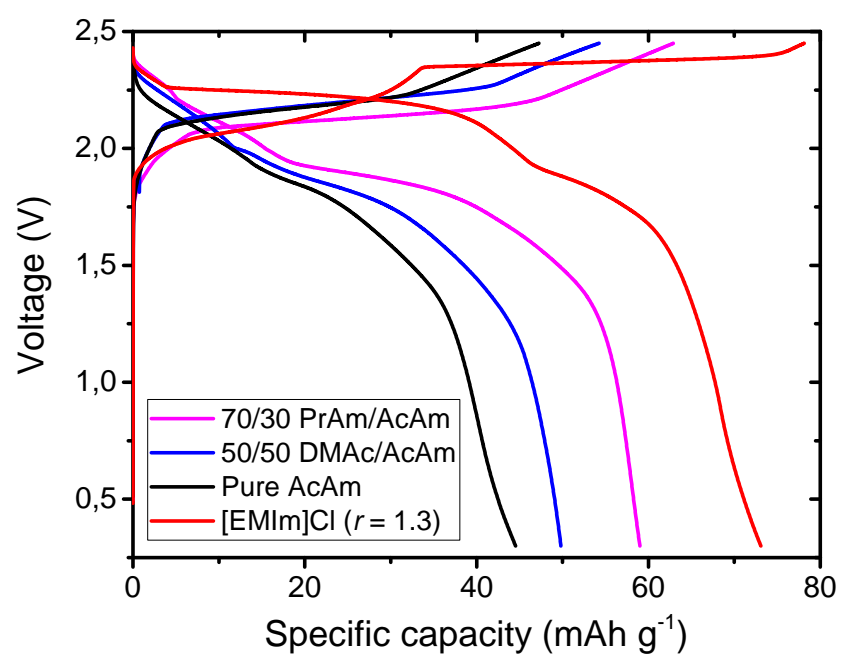

FIGURE 8.14: Typical galvanostatic charge-discharge profiles (tenth cycle, using a current rate of $50 \mathrm{~mA} \mathrm{~g}^{-1}$ ) of Swagelok-type cells built using mixed amide electrolytes, with an $r$ ratio of 1.5, compared with the literature standard [EMIm]Clbased electrolyte. The voltage reported in this plot is the cell potential.

\subsection{Conclusions}

In summary, the work presented in this chapter successfully demonstrates the ability of amide $/ \mathrm{AlCl}_{3}$ mixtures to work as electrolytes for aluminium ion batteries. The large price difference between simple amides and [EMIm] Cl could allow such electrolytes to bring a considerable reduction in the production costs of commercial AIB devices. ${ }^{*}$ Furthermore, this work has shown that diluting the electrolytes with a small quantity of of an appropriate solvent could bring a significant increase in the performance of the device thanks to a substantial reduction of the viscosity of the liquids. The search for a compatible dilutant, however, has proven to be quite challenging due to the high reactivity of the eutectic mixture. Finally, it was also demonstrated that using a mixture of amides can further bring improvement in the performance by adding an element of molecular disorder in the electrolytes. There are still a few issues that require further investigation, such as the behaviour of the electrolytes at high charging and discharging rates as well as more exhaustive studies on the optimal amide ratios. In addition, the search for other replacement compounds can be continued, to be used either as diluting

*The cost per $\mathrm{kg}$ of acetamide is $282 \mathrm{NZ} \$$, compared to $687 \mathrm{NZ} \$ / \mathrm{kg}$ for [EMIm]Cl (SigmaAldrich, September 2018 data). 
agents to the existing systems, or other as new eutectic-forming compounds altogether. All these matters constitute interesting scientific questions, which could be studied in future research.

\subsection{Experimental section}

Acetamide, [EMIm] Cl, $\mathrm{AlCl}_{3}$, dichloromethane, acetonitrile, chloroform, NMP, DMF, toluene, propylene carbonate, tetrahydrofuran, dimethyl sulfoxide, DMAc, NMAc, and PrAm were purchased from Sigma-Aldrich. Molybdenum foil, Al foil and pyrolytic graphite paper were purchased from MTI Corp. Acetamide, [EMIm]Cl, $\mathrm{AlCl}_{3}, \mathrm{NMAc}$, and PrAm were dried by heating at $100{ }^{\circ} \mathrm{C}$ in a vacuum oven for 48 hours. Dichloromethane, acetonitrile, chloroform, NMP, DMF, toluene, propylene carbonate, tetrahydrofuran, dimethyl sulfoxide and DMAc were dried over activated molecular sieves ( $3 \AA$ ) for 48 hours. All the other materials were used as received.

Cyclic voltammetry and galvanostatic charge-discharge experiments were either conducted in a glass cell using a three-electrode configuration, or a Swageloktype cell using a two-electrode configuration. Cyclic voltammetry experiments were run using a potentiostat (Metrohm Autolab PGSTAT128N) using a voltage ramp of $80 \mathrm{mV} \mathrm{s}^{-1}$.

Galvanostatic charge-discharge experiments were performed using a battery analyser system (NEWARE BTS CT-4008-5V10mA-164, MTI Corp.), in the range of 0.3 - $2.45 \mathrm{~V}$, using a rate of $83 \mathrm{~mA} \mathrm{~g}^{-1}$ unless otherwise specified.

\section{Electrolytes preparation}

The previously dried chemicals were transferred into a nitrogen-filled glove box with the $\mathrm{O}_{2}$ and $\mathrm{H}_{2} \mathrm{O}$ levels kept below 1 ppm. Briefly, the electrolytes were synthesised by gradually adding $\mathrm{AlCl}_{3}$ (either 1.1, 1.3 or 1.5 equivalents, depending on the desired molar $r$ ratio) to one equivalent of either [EMIm] Cl, AcAm, DMAc, NMAc, PrAm, or a binary mixture of the aforementioned compounds, under magnetic stirring at room temperature. An exothermic reaction took place, resulting in the formation of viscous liquids. Any residual $\mathrm{AlCl}_{3}$ was further reacted by heating the mixtures to $120^{\circ} \mathrm{C}$ over a hotplate for 1 hour. Dilutions of the electrolytes 
with solvents were performed by directly adding the desired volume fraction to the electrolytes under magnetic stirring. This step was also performed inside the glovebox.

\section{Electrochemical cells assembly}

For measurements in the three-electrode configuration, a sealed, glass electrochemical cell was used, consisting of a glass conical vessel, and a plastic top piece with an O-ring joint. The counter and pseudo-reference electrode consisted of two small sheets of aluminium foil, and the working electrode was either a third sheet of $\mathrm{Al}$ foil or pyrolytic graphite paper. The electrodes were connected via alligator clips to custom-built electrode shafts inserted into the cell and sealed with O-rings. Due to the brittleness of pyrolytic graphite paper, the sheet was sandwiched between two small molybdenum foil sheets, to prevent the alligator clips from damaging it (Figure 8.15). A glassy carbon disk electrode was used as the working electrode to obtain the cyclic voltammograms in Figure 8.5 (right). In order to fill the cell with electrolyte, the sealed vessel was connected to a Schlenk line, and

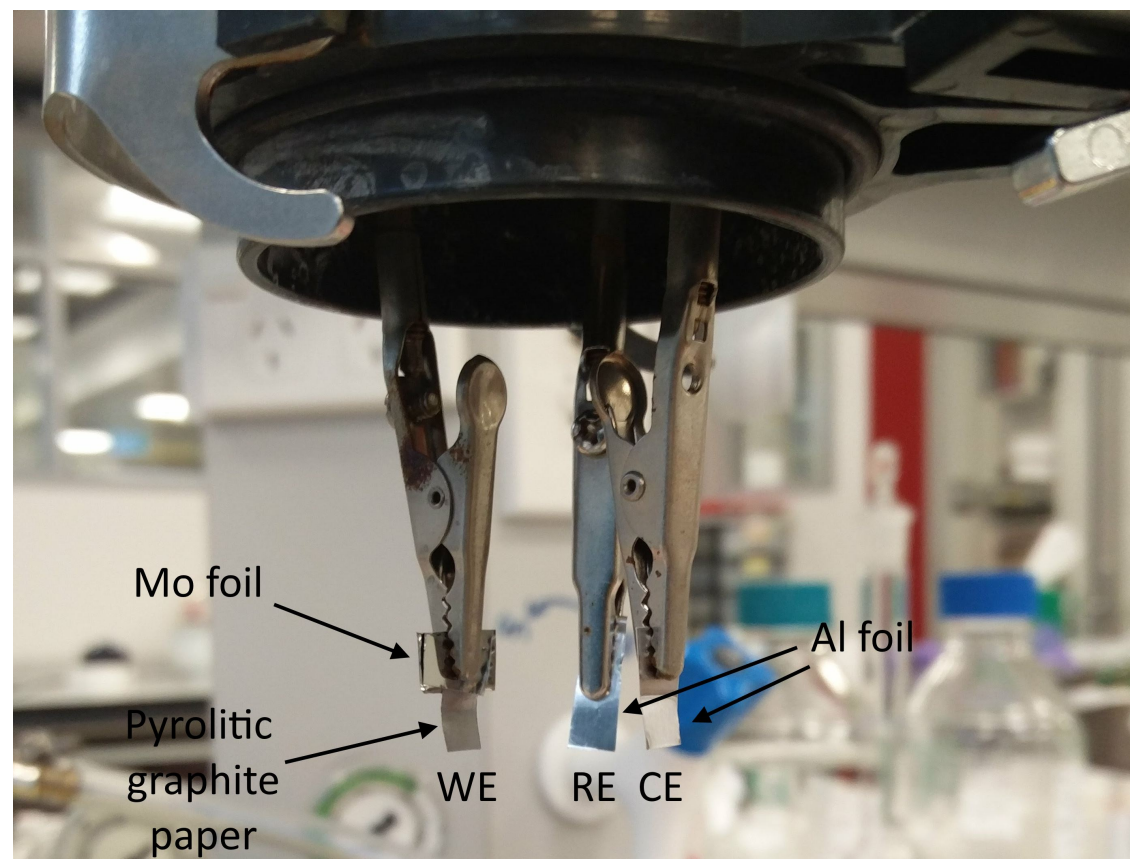

FIGURE 8.15: Digital camera image of a partially assembled three-electrode cell setup, used to perform some of the electrochemical tests described in this chapter. Adapted by permission of The Royal Society of Chemistry. 
was flushed with nitrogen by performing three vacuum- $\mathrm{N}_{2}$ cycles. Then, approximately $3 \mathrm{~mL}$ of one of the electrolytes were taken out of the glovebox in a sealed vial with a rubber septum cap and immediately transferred into the electrochemical cell using a syringe. The as-prepared cell was then used for the electrochemical tests.

For measurements in the two-electrode configuration, prototype batteries were assembled using a custom-built Swagelok-type cell consisting of a cylindrical PEEK casing with an inner diameter of $12 \mathrm{~mm}$ and two Mo rods as the current collectors (see also Section 3.4.2). All the components of the device were first baked at 100 ${ }^{\circ} \mathrm{C}$ in a vacuum oven for at least 2 hours to remove any residual water, then immediately transferred inside a nitrogen-filled glovebox with the $\mathrm{O}_{2}$ and $\mathrm{H}_{2} \mathrm{O}$ levels kept below $1 \mathrm{ppm}$. Pyrolytic graphite paper sheets were cut into $11 \mathrm{~mm}$ diameter discs to be used as a cathode. High purity Al foil discs (11 mm diameter) were used as the anode. Glass microfibre (GF/D) discs with $12 \mathrm{~mm}$ diameters, soaked in approximately $300 \mu \mathrm{L}$ of the electrolyte, were used as separators. The devices were then wrapped with Parafilm as an additional moisture barrier, and taken outside the glovebox for electrochemical testing.

\section{Bibliography}

${ }^{1}$ T. Jiang, M. J. Chollier Brym, G. Dubé, A. Lasia, and G. M. Brisard, “Electrodeposition of aluminium from ionic liquids: Part I-electrodeposition and surface morphology of aluminium from aluminium chloride ( $\mathrm{AlCl}$ )-1-ethyl-3-methylimidazolium chloride ([EMIm]Cl) ionic liquids," Surface and Coatings Technology, vol. 201, pp. 1-9, Sept. 2006.

${ }^{2}$ K. V. Kravchyk, S. Wang, L. Piveteau, and M. V. Kovalenko, "Efficient Aluminum Chloride-Natural Graphite Battery," Chemistry of Materials, vol. 29, pp. 4484-4492, May 2017.

${ }^{3} \mathrm{~S}$. Gu, H. Wang, C. Wu, Y. Bai, H. Li, and F. Wu, "Confirming reversible Al3+ storage mechanism through intercalation of $\mathrm{Al} 3+$ into $\mathrm{V} 2 \mathrm{O} 5$ nanowires in a rechargeable aluminum battery," Energy Storage Materials, vol. 6, pp. 9-17, Jan. 2017.

${ }^{4}$ M. Chiku, H. Takeda, S. Matsumura, E. Higuchi, and H. Inoue, “Amorphous Vanadium Oxide/Carbon Composite Positive Electrode for Rechargeable Aluminum Battery," ACS Applied Materials E Interfaces, vol. 7, pp. 24385-24389, Nov. 2015.

${ }^{5}$ H. Jiao, C. Wang, J. Tu, D. Tian, and S. Jiao, “A rechargeable Al-ion battery: Al/molten AlCl 3 -urea/graphite," Chemical Communications, vol. 53, no. 15, pp. 2331-2334, 2017.

${ }^{6}$ Y. Song, S. Jiao, J. Tu, J. Wang, Y. Liu, H. Jiao, X. Mao, Z. Guo, and D. J. Fray, “A long-life rechargeable Al ion battery based on molten salts," Journal of Materials Chemistry A, vol. 5, pp. 1282-1291, Jan. 2017. 
${ }^{7}$ N. Canever, N. Bertrand, and T. Nann, "Acetamide: a low-cost alternative to alkyl imidazolium chlorides for aluminium-ion batteries," Chemical Communications, vol. 54, no. 83, pp. 1172511728, 2018.

${ }^{8}$ A. P. Abbott, C. A. Eardley, N. R. S. Farley, G. A. Griffith, and A. Pratt, “Electrodeposition of aluminium and aluminium/platinum alloys from $\mathrm{AlCl}$ /benzyltrimethylammonium chloride room temperature ionic liquids," Journal of Applied Electrochemistry, vol. 31, pp. 1345-1350, Dec. 2001.

${ }^{9}$ A. Kitada, K. Nakamura, K. Fukami, and K. Murase, " $\mathrm{AlCl}_{3}$-dissolved Diglyme as Electrolyte for Room-Temperature Aluminum Electrodeposition," Electrochemistry, vol. 82, no. 11, pp. 946-948, 2014.

${ }^{10}$ A. P. Abbott, R. C. Harris, Y.-T. Hsieh, K. S. Ryder, and I.-W. Sun, "Aluminium electrodeposition under ambient conditions," Physical Chemistry Chemical Physics, vol. 16, pp. 14675-14681, June 2014.

${ }^{11}$ M. Li, B. Gao, C. Liu, W. Chen, Z. Shi, X. Hu, and Z. Wang, "Electrodeposition of aluminum from AlCl3/acetamide eutectic solvent," Electrochimica Acta, vol. 180, pp. 811-814, Oct. 2015.

${ }^{12}$ Y. Fang, K. Yoshii, X. Jiang, X. G. Sun, T. Tsuda, N. Mehio, and S. Dai, "An AlCl3 based ionic liquid with a neutral substituted pyridine ligand for electrochemical deposition of aluminum," Electrochimica Acta, vol. 160, pp. 82-88, Apr. 2015.

${ }^{13}$ H. M. A. Abood, A. P. Abbott, A. D. Ballantyne, and K. S. Ryder, "Do all ionic liquids need organic cations? Characterisation of [AlCl2-nAmide]+AlCl4- and comparison with imidazolium based systems," Chemical Communications, vol. 47, pp. 3523-3525, Mar. 2011.

${ }^{14}$ P. K. Lai and M. Skyllas-Kazacos, "Electrodeposition of aluminium in aluminium chloride/1methyl-3-ethylimidazolium chloride," Journal of Electroanalytical Chemistry and Interfacial Electrochemistry, vol. 248, pp. 431-440, July 1988.

${ }^{15}$ G. Antonio Elia, I. Hasa, G. Greco, T. Diemant, K. Marquardt, K. Hoeppner, R. Jürgen Behm, A. Hoell, S. Passerini, and R. Hahn, "Insights into the reversibility of aluminum graphite batteries," Journal of Materials Chemistry A, vol. 5, no. 20, pp. 9682-9690, 2017.

${ }^{16}$ M.-C. Lin, M. Gong, B. Lu, Y. Wu, D.-Y. Wang, M. Guan, M. Angell, C. Chen, J. Yang, B.-J. Hwang, and H. Dai, "An ultrafast rechargeable aluminium-ion battery," Nature, vol. 520, pp. 324-328, Apr. 2015.

${ }^{17}$ A. S. Childress, P. Parajuli, J. Zhu, R. Podila, and A. M. Rao, "A Raman spectroscopic study of graphene cathodes in high-performance aluminum ion batteries," Nano Energy, vol. 39, pp. 6976, Sept. 2017.

${ }^{18}$ P. Bhauriyal, A. Mahata, and B. Pathak, "The staging mechanism of AlCl4- intercalation in a graphite electrode for an aluminium-ion battery," Physical Chemistry Chemical Physics, vol. 19, pp. 7980-7989, Mar. 2017.

${ }^{19}$ L. Zhang, L. Chen, H. Luo, X. Zhou, and Z. Liu, "Large-Sized Few-Layer Graphene Enables an Ultrafast and Long-Life Aluminum-Ion Battery," Advanced Energy Materials, vol. 7, p. 1700034, Aug. 2017.

${ }^{20}$ G. Greco, D. Tatchev, A. Hoell, M. Krumrey, S. Raoux, R. Hahn, and G. Antonio Elia, “Influence of the electrode nano/microstructure on the electrochemical properties of graphite in aluminum batteries," Journal of Materials Chemistry A, vol. 6, no. 45, pp. 22673-22680, 2018.

${ }^{21}$ S. Wang, S. Jiao, W.-L. Song, H.-S. Chen, J. Tu, D. Tian, H. Jiao, C. Fu, and D.-N. Fang, “A novel dual-graphite aluminum-ion battery," Energy Storage Materials, vol. 12, pp. 119-127, 2018. 
${ }^{22}$ D.-Y. Wang, C.-Y. Wei, M.-C. Lin, C.-J. Pan, H.-L. Chou, H.-A. Chen, M. Gong, Y. Wu, C. Yuan, M. Angell, Y.-J. Hsieh, Y.-H. Chen, C.-Y. Wen, C.-W. Chen, B.-J. Hwang, C.-C. Chen, and H. Dai, "Advanced rechargeable aluminium ion battery with a high-quality natural graphite cathode," Nature Communications, vol. 8, p. 14283, Feb. 2017.

${ }^{23}$ H. Chen, H. Xu, S. Wang, T. Huang, J. Xi, S. Cai, F. Guo, Z. Xu, W. Gao, and C. Gao, “Ultrafast all-climate aluminum-graphene battery with quarter-million cycle life," Science Advances, vol. 3, p. eaao7233, Dec. 2017.

${ }^{24}$ J. Newman and K. E. Thomas-Alyea, Electrochemical Systems. John Wiley \& Sons, third ed., Nov. 2012.

${ }^{25}$ A. Boisset, S. Menne, J. Jacquemin, A. Balducci, and M. Anouti, “Deep eutectic solvents based on $\mathrm{N}$-methylacetamide and a lithium salt as suitable electrolytes for lithium-ion batteries," Physical Chemistry Chemical Physics, vol. 15, pp. 20054-20063, Nov. 2013.

${ }^{26}$ A. Endo, M. Miyake, and T. Hirato, "Electrodeposition of Aluminum from 1,3-Dimethyl-2Imidazolidinone/ AlCl3 baths," Electrochimica Acta, vol. 137, pp. 470-475, Aug. 2014.

${ }^{27}$ M. Li, B. Gao, C. Liu, W. Chen, Z. Wang, Z. Shi, and X. Hu, "AlCl3/amide ionic liquids for electrodeposition of aluminum," Journal of Solid State Electrochemistry, vol. 21, pp. 469-476, Feb. 2017.

${ }^{28}$ E. W. Castner, J. F. Wishart, and H. Shirota, "Intermolecular Dynamics, Interactions, and Solvation in Ionic Liquids," Accounts of Chemical Research, vol. 40, pp. 1217-1227, Nov. 2007.

${ }^{29}$ S. Zahn, F. Uhlig, J. Thar, C. Spickermann, and B. Kirchner, "Intermolecular Forces in an Ionic Liquid ([Mmim][Cl]) versus Those in a Typical Salt $(\mathrm{NaCl}), "$ Angewandte Chemie International Edition, vol. 47, no. 19, pp. 3639-3641, 2008. 
Chapter 9

Conclusions and outlook 



\subsection{Conclusions}

In this PhD work, the application of nanostructured materials as cathode materials for aluminium-ion batteries, and the use of low-cost organic compounds as an alternative to the expensive organic chloride salts in AIBs electrolytes, have been investigated. A brief summary of the most interesting new findings is outlined in this section.

\subsubsection{Nanostructured cathodes}

Although none of the nanomaterials fabricated during this project have produced a significant breakthrough in battery performance, some interesting discoveries have been made through their study:

- Although the performance of $\mathrm{V}_{2} \mathrm{O}_{5}$ nanofibres was somewhat underwhelming, the material provided a useful preliminary demonstration that nanomaterials can indeed yield better electrochemical performance than the corresponding bulk materials. However, some discrepancies with previous literature reports have been highlighted in the experiments performed, as the specific discharge capacities obtained in the galvanostatic cycling tests performed are notably lower than those reported by their authors.

- Due to instrumental limitations, only semi-graphitic carbon nanofibres could be fabricated and tested. Such materials, if used "as-prepared", have been shown to work mainly through a capacitive or pseudo-capacitive energy storage mechanism, with no significant intercalation or deintercalation processes taking place. This initially led to the conclusion that only highly graphitic materials could allow the insertion of $\mathrm{AlCl}_{4}^{-}$ions.

- Experimental evidence has also revealed that the low graphitic character, correlated with a high quantity of surface defects, and the high surface area of CNF can cause a reduction of the useful electrolyte voltage window by promoting the decomposition of the electrolyte at lower potentials, which in turn causes the formation of a solid-electrolyte interphase. Therefore, if a carbon material were to be used as a cathode for AIBs, it must also be highly graphitic to allow an efficient intercalation-based energy storage mechanism and avoid the unwanted SEI formation. 
- Core-sheath $\mathrm{C} / \mathrm{V}_{2} \mathrm{O}_{5}$ nanofibres show an interesting behaviour when used as cathodes for AIBs, indicating the presence of a mixed-ion intercalation mechanism made possible by the synergistic effects between the core and sheath layers. This effect is short-lived, however, due to the rapid degradation of the sheath material.

- The synergistic effect observed for the core-sheath $\mathrm{C} / \mathrm{V}_{2} \mathrm{O}_{5}$ nanofibres appears to not take place in the case of a layered electrode structure due to the poor adhesion of the $\mathrm{V}_{2} \mathrm{O}_{5}$ active material to the CNF-based current collector. This also caused the electrode to yield overall poor, unreliable performance.

\subsubsection{Alternative electrolytes}

A series of low-cost alternatives to alkyl-imidazolium chlorides was successfully found in acetamide and a few other simple amide analogues. Unlike other proposed alternative electrolytes, the amide- $\mathrm{AlCl}_{3}$ mixture can offer good performance at room temperature, and could therefore constitute a valid alternative to the state-of-the-art [EMIm] $\mathrm{Cl}-\mathrm{AlCl}_{3}$ electrolyte. Dilution with appropriate solvents was found to help increase the performance of the electrolytes by reducing their viscosities. However, finding an appropriate solvent can be a challenging task, due to the possibility of unwanted reactions with the active electrolyte species and reduction of the usable electrochemical window. Another strategy to reduce the viscosity of the electrolytes is to add an element of disorder by using mixture of amides instead of pure compounds: this could potentially result in less viscous liquids thanks to the formation of weaker ionic pairs.

\subsection{Future work}

The research findings outlined in this project could be further expanded with future research:

- The poor performance and irreversibility of the intercalation reaction in $\mathrm{V}_{2} \mathrm{O}_{5}$ could be studied to find ways to mitigate such issues and bring the performance of the material closer to its theoretical value and allow longer device lives. 
- The performance of highly graphitic CNF as cathode could be studied to confirm that only graphitic carbons can effectively allow intercalation and deintercalation of $\mathrm{AlCl}_{4}^{-}$ions. This would require using higher carbonisation temperatures through instrumentation available at other universities or research institutes.

- The phenomenon of SEI formation in AIBs needs to be studied in further depth: characterisation of the film could be useful to identify its composition and its impact on battery performance, and in-depth materials characterisation would also help to identify what factors are exactly the cause for its formation.

- The rapid degradation of the $\mathrm{V}_{2} \mathrm{O}_{5}$ layer in core-sheath nanofibres could be studied to find ways of slowing down the process while maintaining the synergistic effect of the material for a longer number of cycles. Alternatively, new material morphologies, or different materials altogether, could also be studied to find a cathode that allows a multiple-ion intercalation mechanism in a similar fashion, but with higher stability.

- The physical properties of amide/ $\mathrm{AlCl}_{3}$ systems need to be further investigated. Key measurements such as conductivity and viscosity need to be performed, and characterisation of the chemical species in the mixture could help obtaining a better understanding of the systems. Additional studies on solvent dilution of the electrolytes could also be performed to investigate the mechanisms behind the performance enhancements. 

Appendix A

Supplementary data 


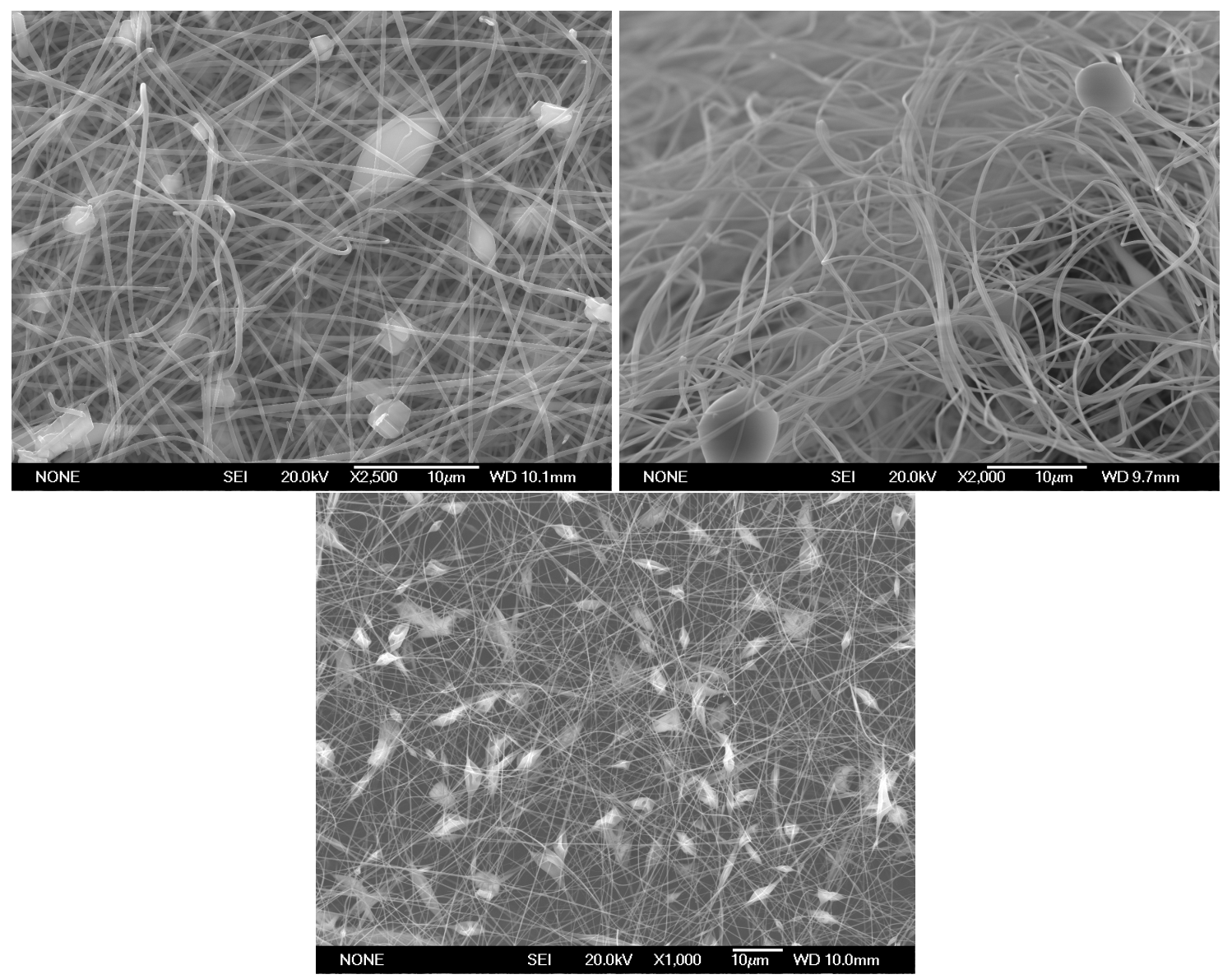

FIGURE A.1: SEM micrographs of failed attempts at electrospinning vanadium precursor/PVP nanofibres using different precursor compounds: vanadyl acetylacetonate (top left), peroxovanadates (top right), and vanadium oxytriethoxide (bottom). 

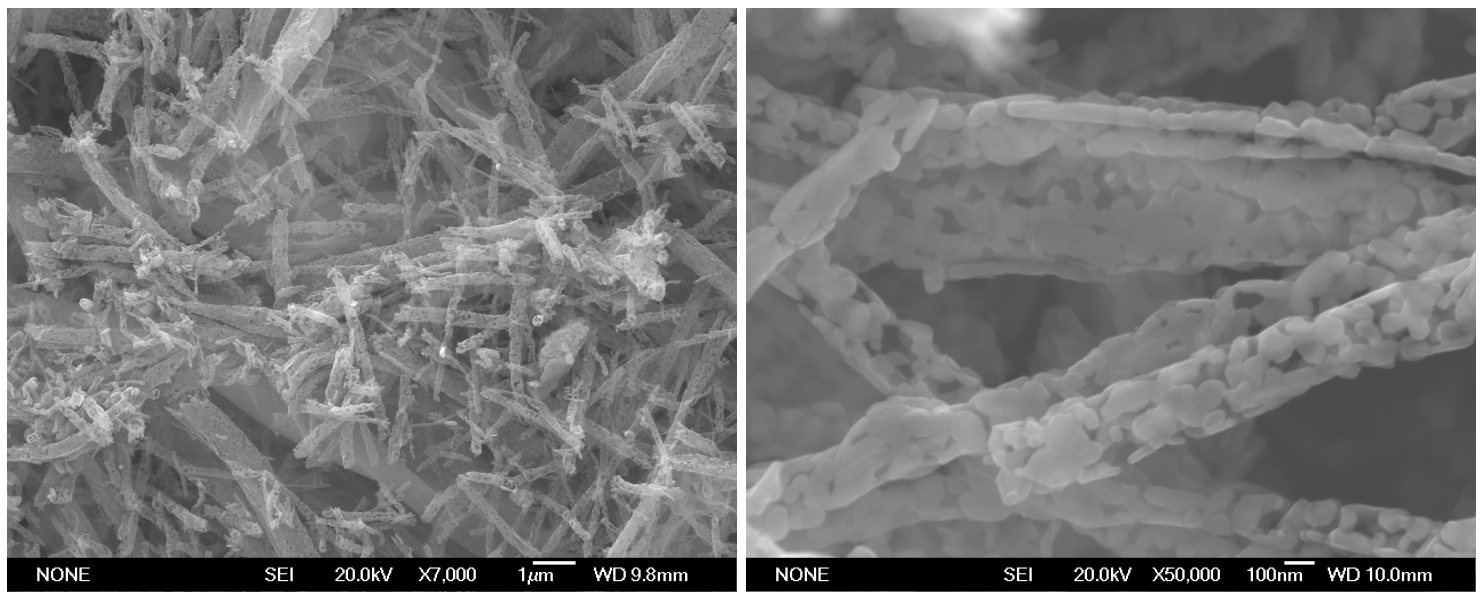

FIGURE A.2: SEM micrographs of electrospun $\mathrm{V}_{2} \mathrm{O}_{5}$ nanofibres prepared using vanadium oxytriethoxide as precursor.

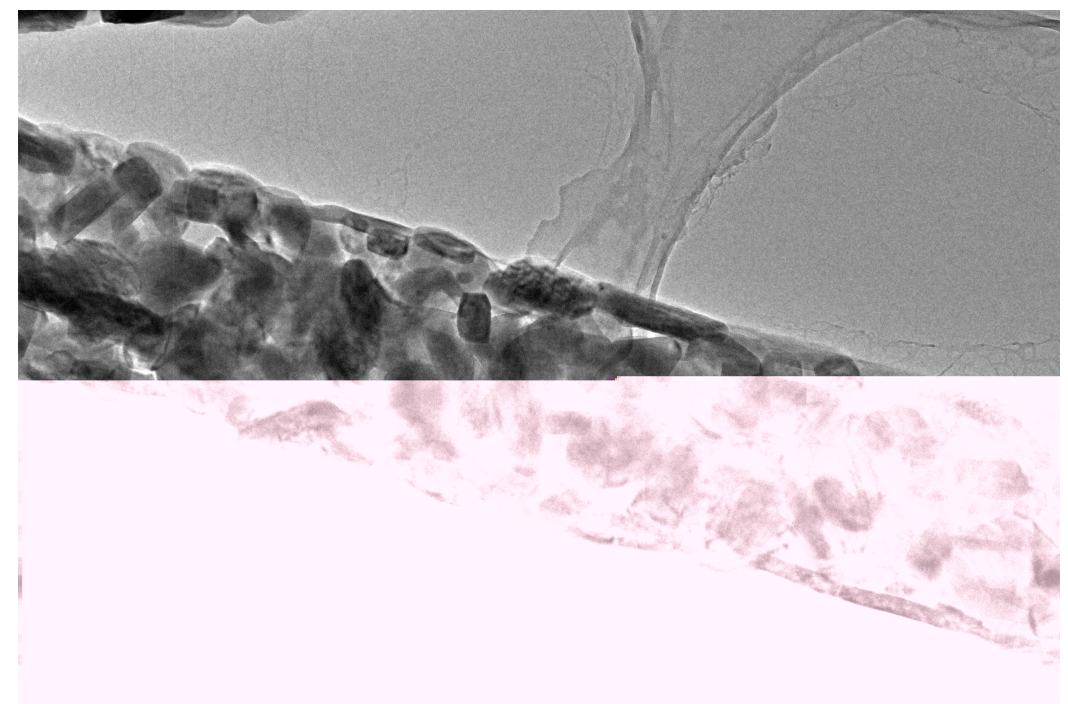

FIGURE A.3: TEM micrograph of a single $\mathrm{V}_{2} \mathrm{O}_{5}$ nanofibre. 

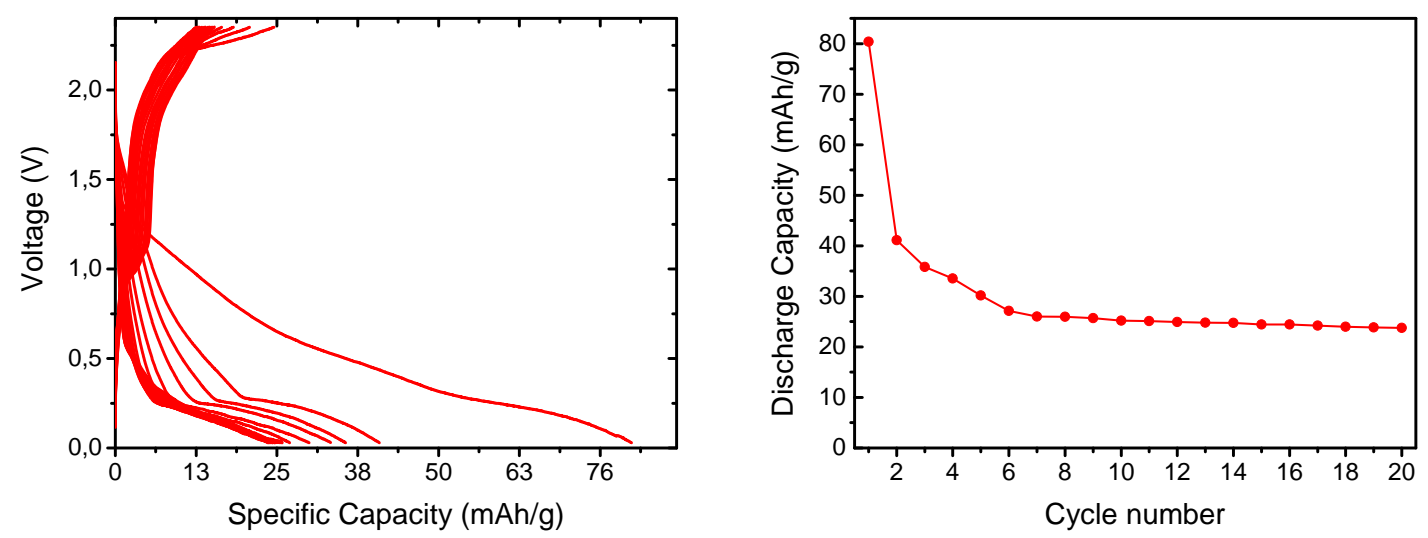

FIGURE A.4: (Left) Galvanostatic cycling profiles of a Swagelok-type cell built using $\mathrm{V}_{2} \mathrm{O}_{5}$ NFs as cathode, using an upper voltage cutoff of $2.35 \mathrm{~V}$. The voltage reported in this plot is the cell potential. (Right) Specific discharge capacities obtained by the galvanostatic charge-discharge experiment.
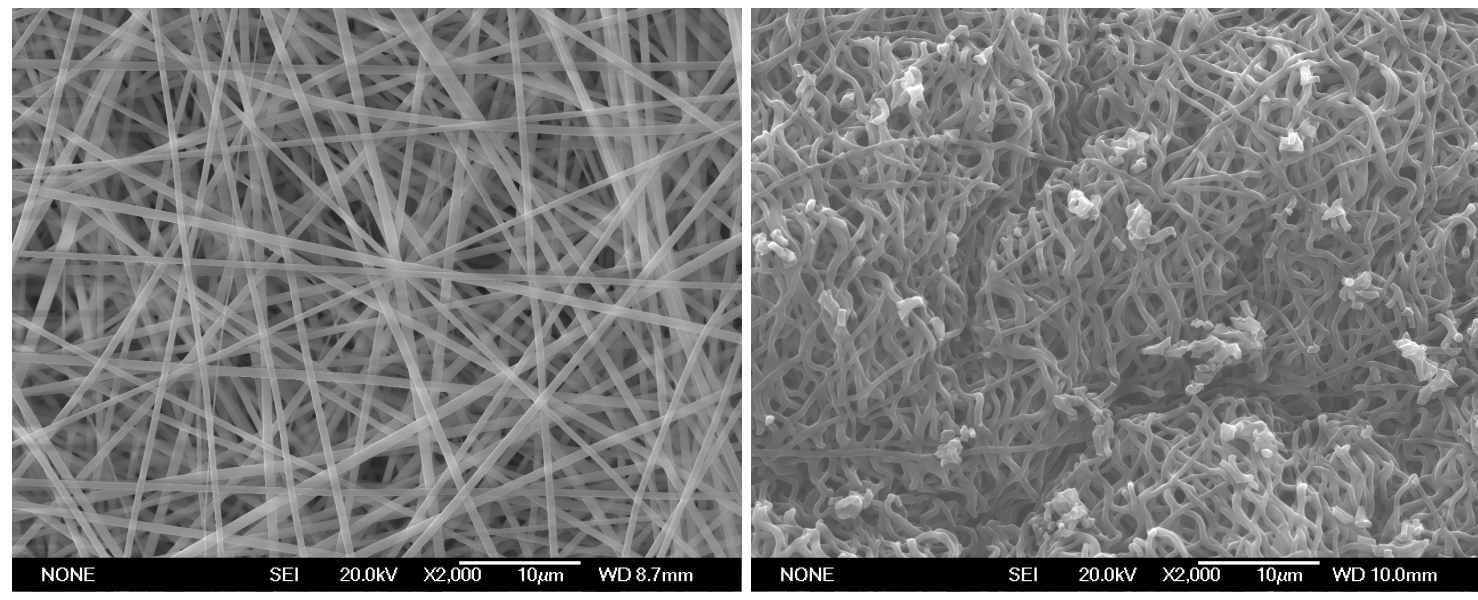

FIGURE A.5: SEM micrographs of a failed attempt at fabricating carbon nanofibres from a PVP precursor: as-spun PVP nanofibres (left) and after stabilisation and carbonisation (right). 

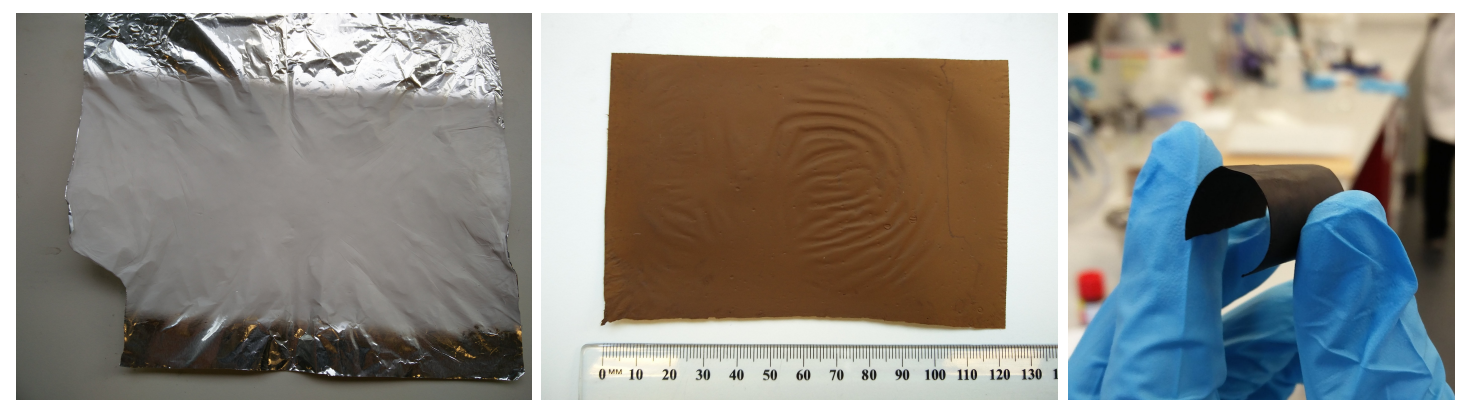

FIGURE A.6: Digital camera images showing the appearance of PAN-based carbon nanofibres at each step of the fabrication process: as-electrospun PAN nanofibres on aluminium foil (left), stabilised PAN nanofibres (center), and finished product (right).
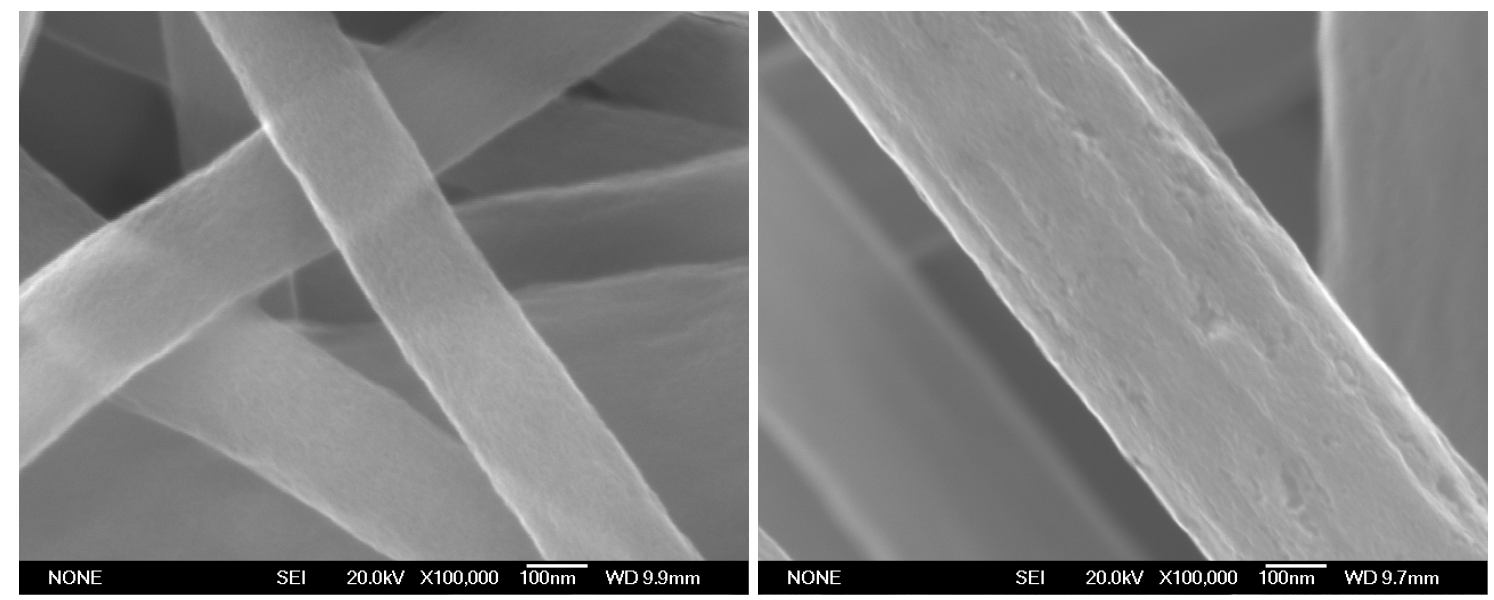

FIGURE A.7: High magnification SEM micrographs of carbon nanofibres carbonised at $900{ }^{\circ} \mathrm{C}$ (left) and $1400{ }^{\circ} \mathrm{C}$ (right). 

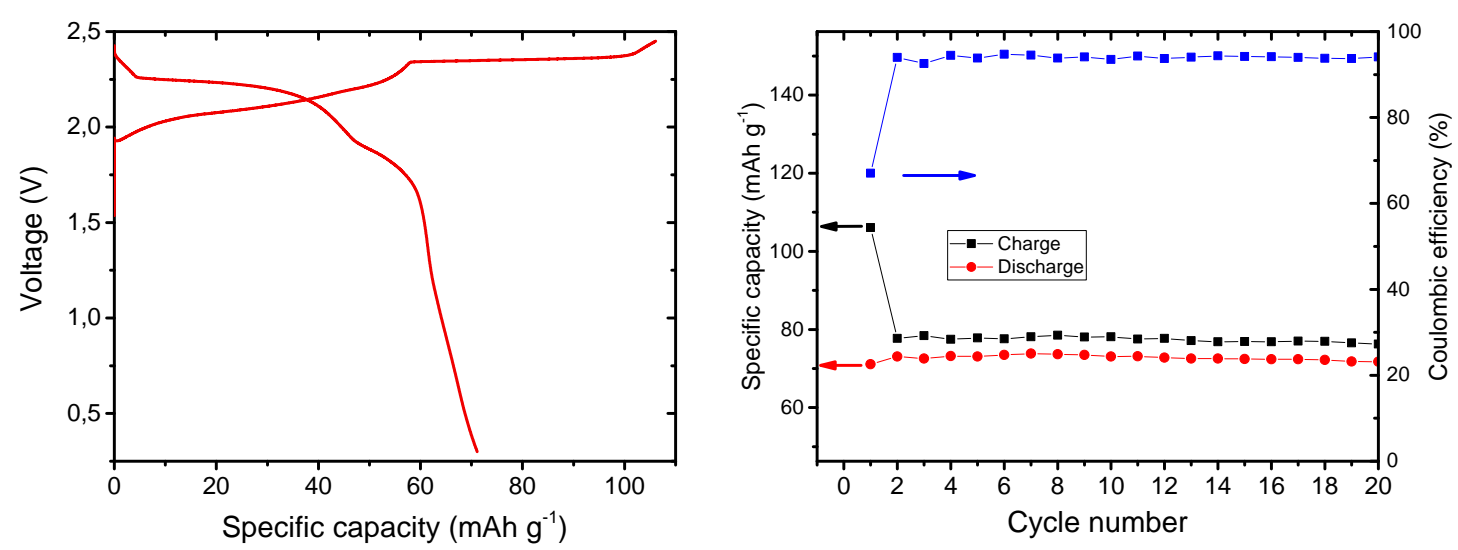

FIGURE A.8: (Left) Galvanostatic profiles relative to the first cycle $\left(50 \mathrm{~mA} \mathrm{~g}^{-1}\right)$ of a Swagelok-type built using pyrolytic graphite paper, showing low coulombic efficiency. The voltage reported in this plot is the cell potential. (Right) Specific capacities and coulombic efficiencies of a typical galvanostatic charge-discharge test $\left(20\right.$ cycles at a current rate of $50 \mathrm{~mA} \mathrm{~g}^{-1}$ ) of the same device.
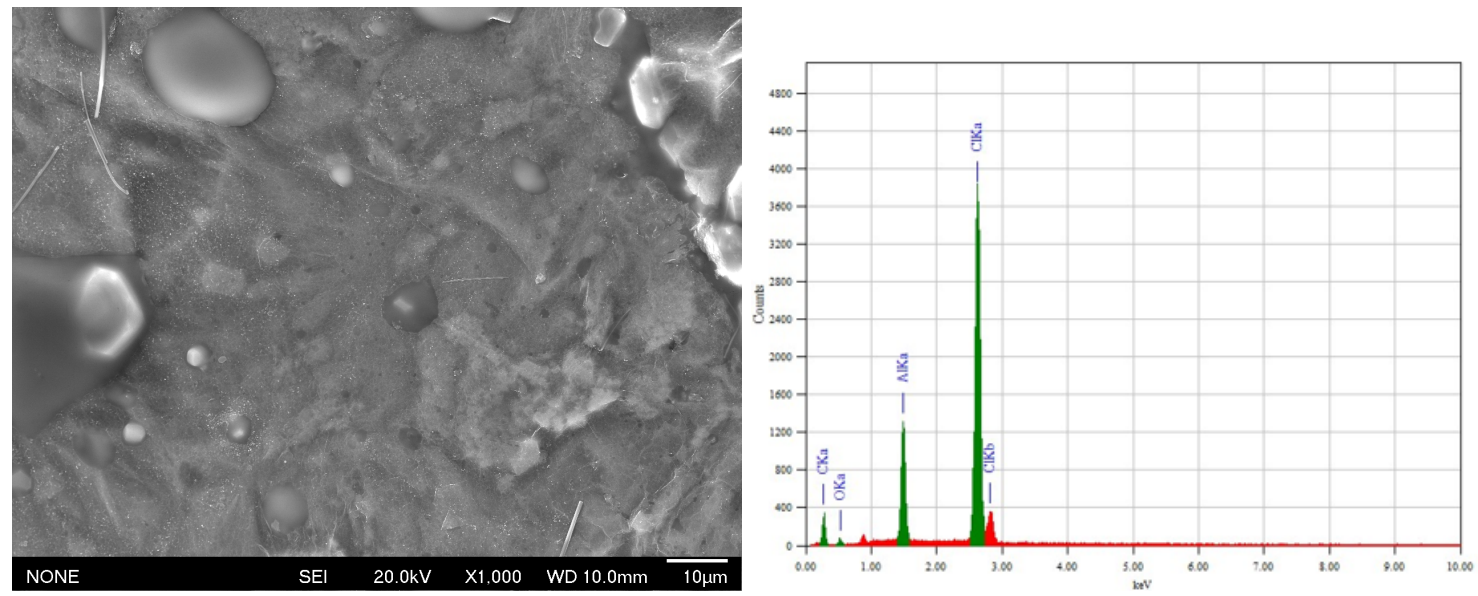

FIGURE A.9: SEM micrograph (left) and EDX spectrum (right) of pyrolytic graphite paper, after a typical galvanostatic charge-discharge test (20 cycles at a current rate of $50 \mathrm{~mA} \mathrm{~g}^{-1}$ ). 


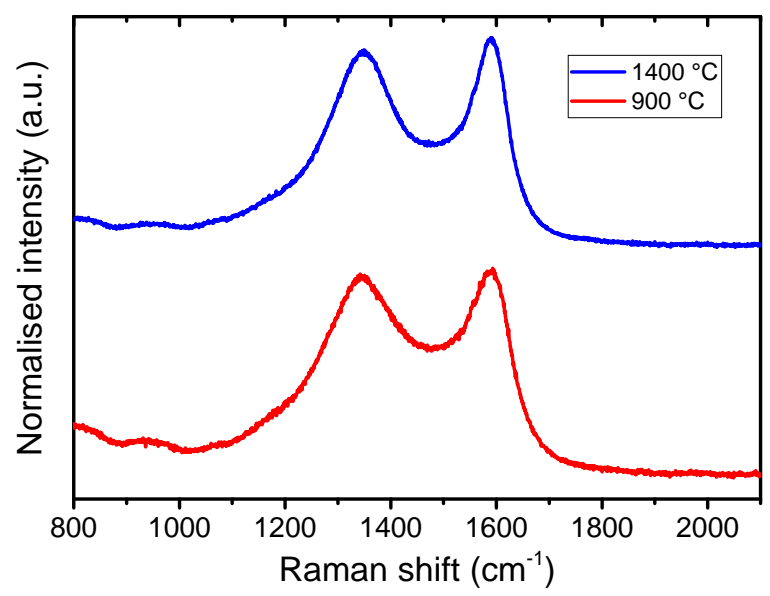

FIGURE A.10: Raman spectra of carbon nanofibres carbonised at different temperatures.
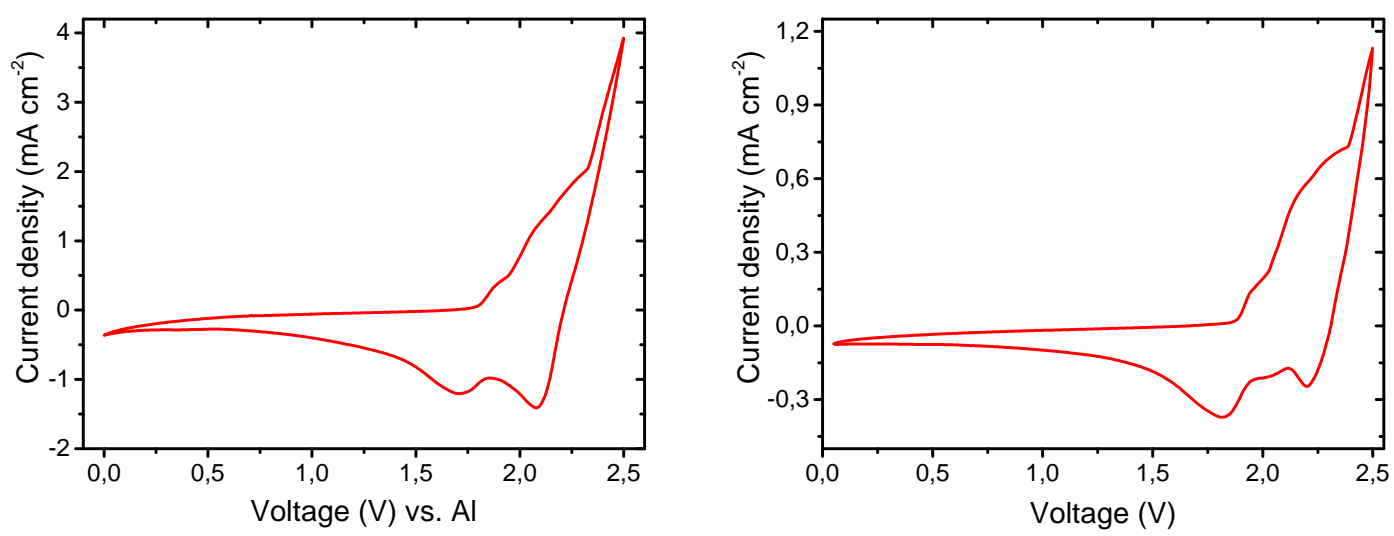

FIGURE A.11: Cyclic voltammograms (third cycle) of cells built using pyrolytic graphite paper as cathode, in two different configurations: three electrode setup (left graph: Al foil as counter and reference electrodes, $80 \mathrm{mV} \mathrm{s}^{-1}$ ), and Swageloktype cell (right graph; $10 \mathrm{mV} \mathrm{s}^{-1}$ ). Both cells use a mixture of $\mathrm{AlCl}_{3}$ and [EMIm]Cl (molar ratio $=1.3$ ) as electrolyte. The current densities are referred to the projected area of the working electrode/cathode. The voltage reported in the left plot is measured against an Al foil pseudo-reference electrode. The voltage in the right plot is the cell potential. It is also worth noting that, regardless of differences in relative intensities of the redox peaks, the deviation in potential values between the two cell geometries is mostly negligible. 


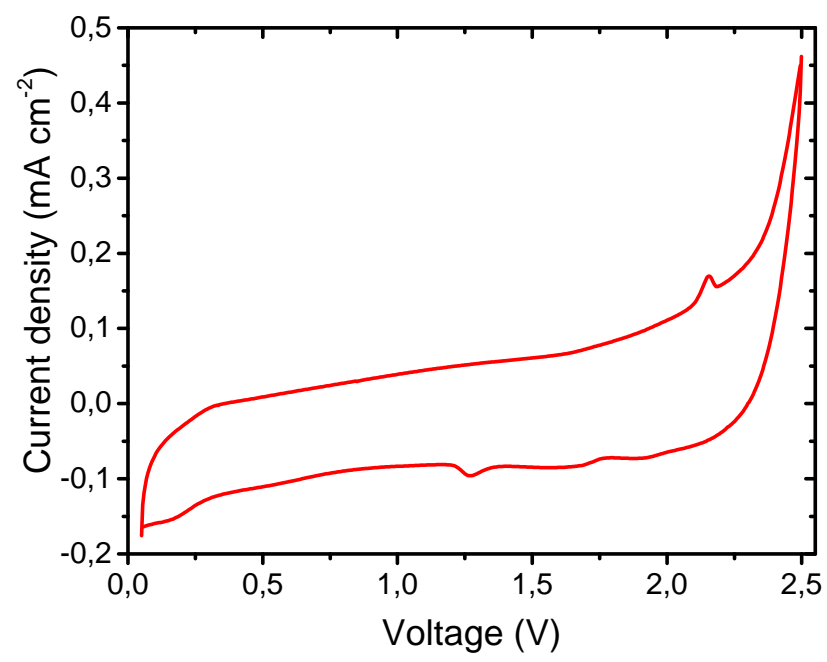

FIGURE A.12: Cyclic voltammogram (third cycle) of a Swagelok-type cell built using carbon nanofibres (carbonised at $1400{ }^{\circ} \mathrm{C}$ ) as cathode, after a long (about $4000 \mathrm{mAh} \mathrm{g}^{-1}$ ) galvanostatic charging step. The current density is referred to the projected cathode area; The voltage reported in this plot is the cell potential.
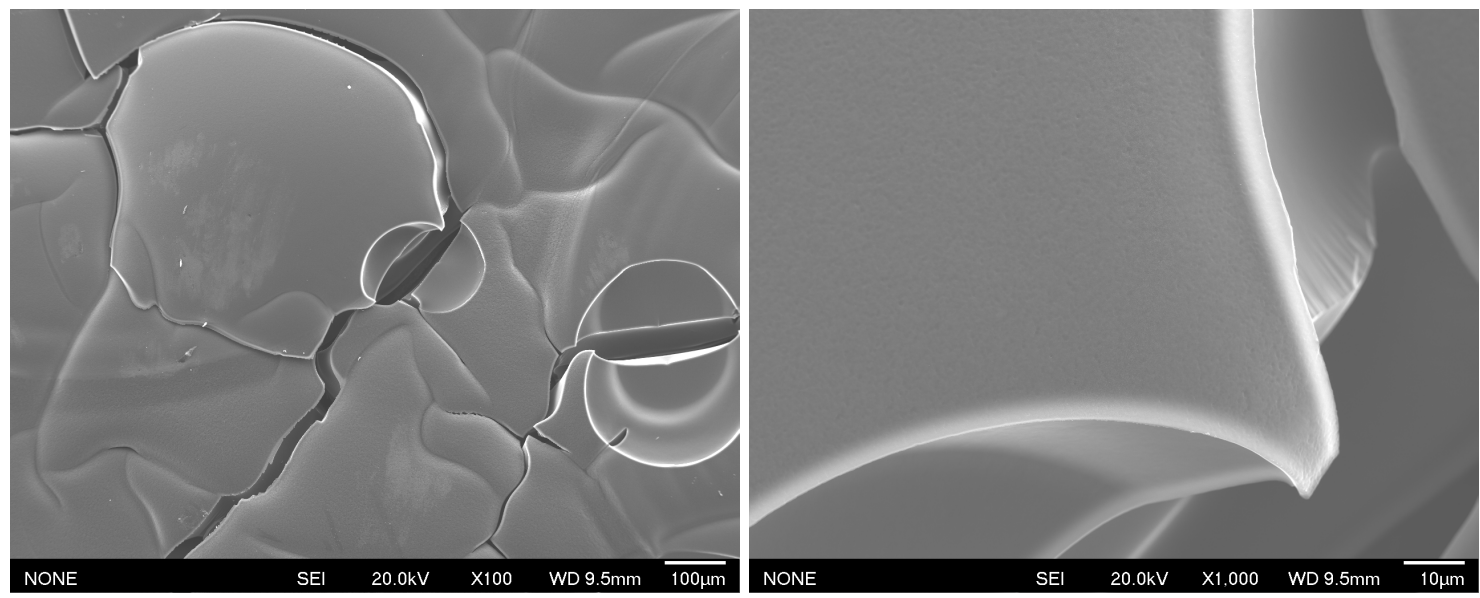

FIGURE A.13: SEM micrographs of the PAN-derived carbon film, showing its monolithic structure. 

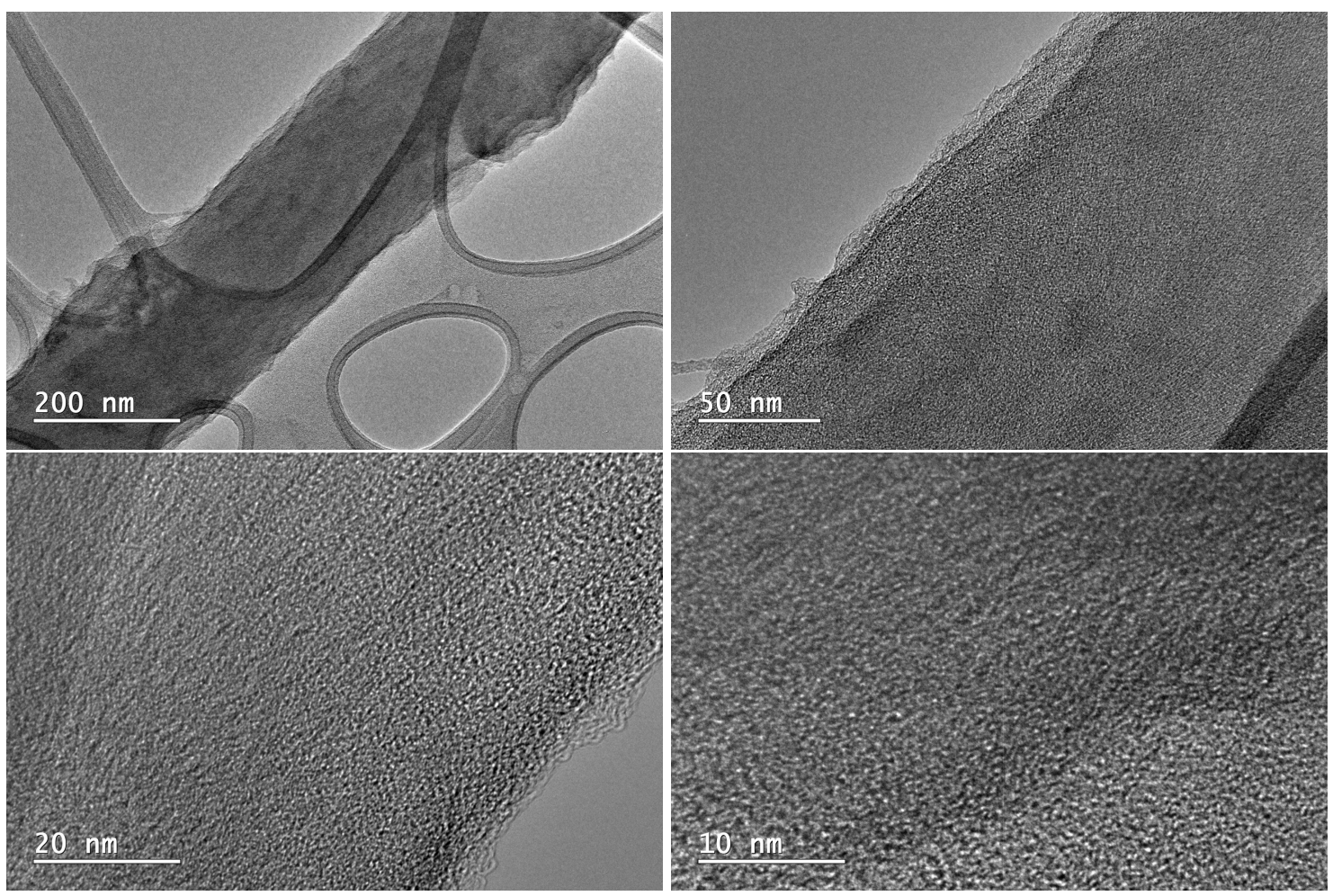

FIGURE A.14: HRTEM micrographs of carbon nanofibres prepared without metal catalysts (carbonised at $1400^{\circ} \mathrm{C}$ ).
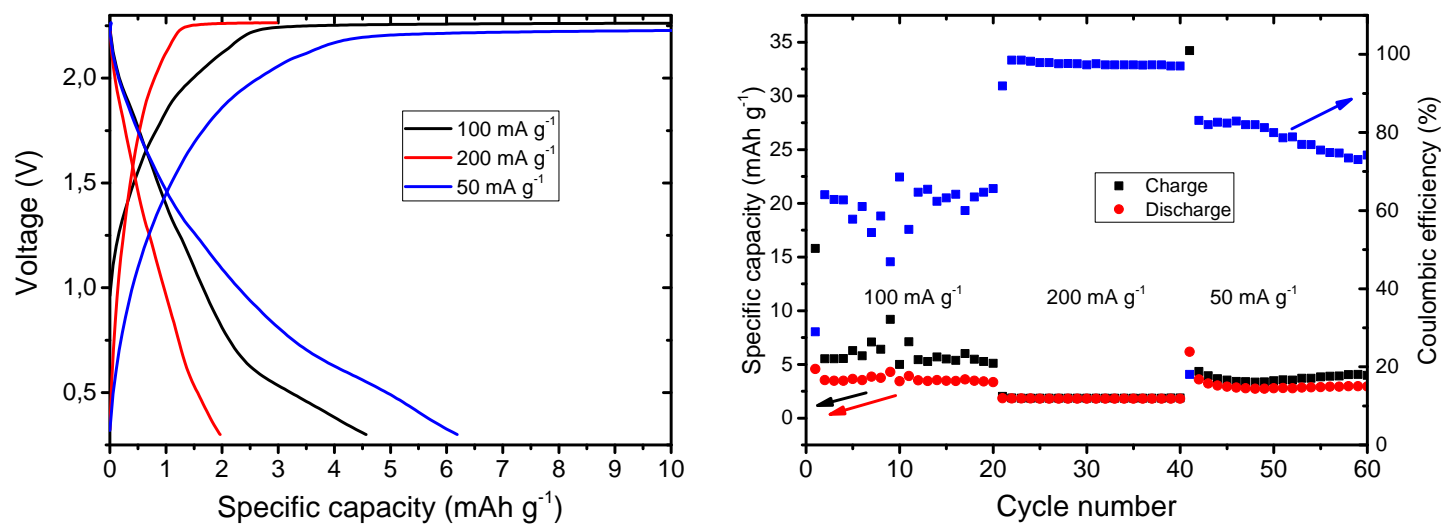

FIGURE A.15: (Left) Typical galvanostatic charge-discharge profiles at various current rates of a Swagelok-type cell built using ferrocene-enhanced carbon nanofibres as cathode. (Right) Specific capacities and coulombic efficiencies of a typical galvanostatic charge-discharge test of a Swagelok-type cell built using ferrocene-enhanced carbon nanofibres as cathode. The voltage reported in this plot is the cell potential. 

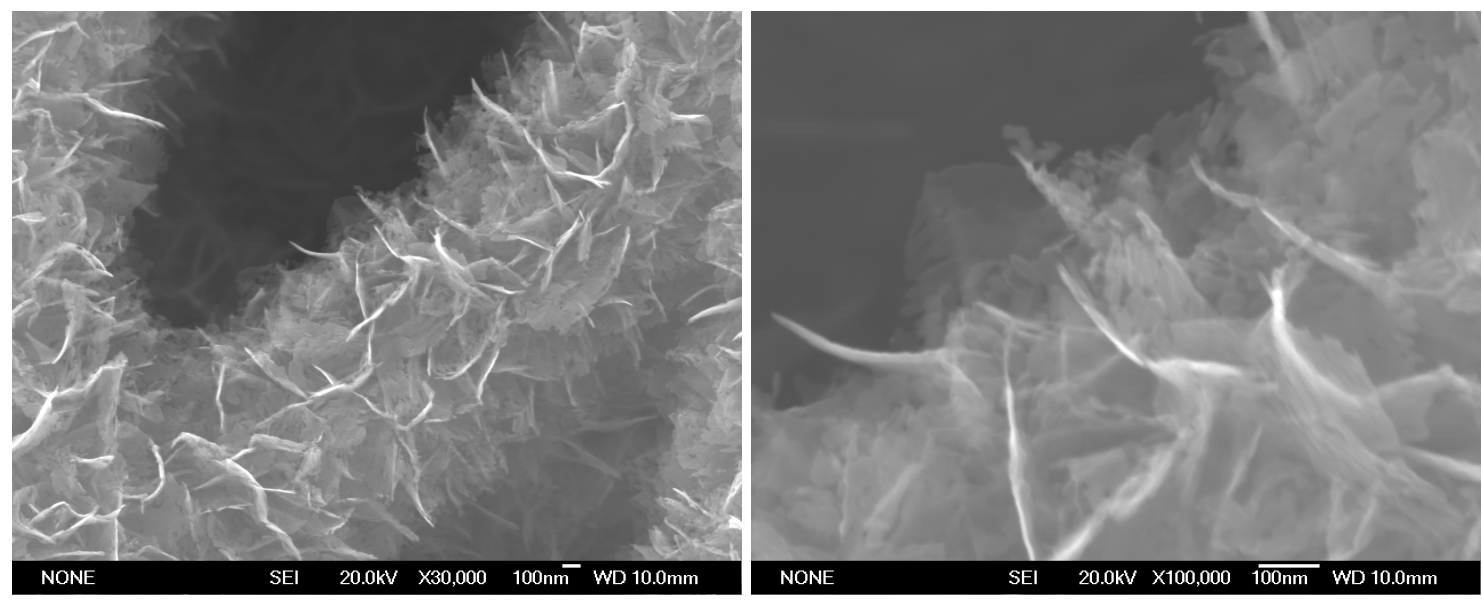

FIGURE A.16: SEM micrographs of core-sheath $\mathrm{C} / \mathrm{V}_{2} \mathrm{O}_{5}$ nanofibres, after the final annealing step.

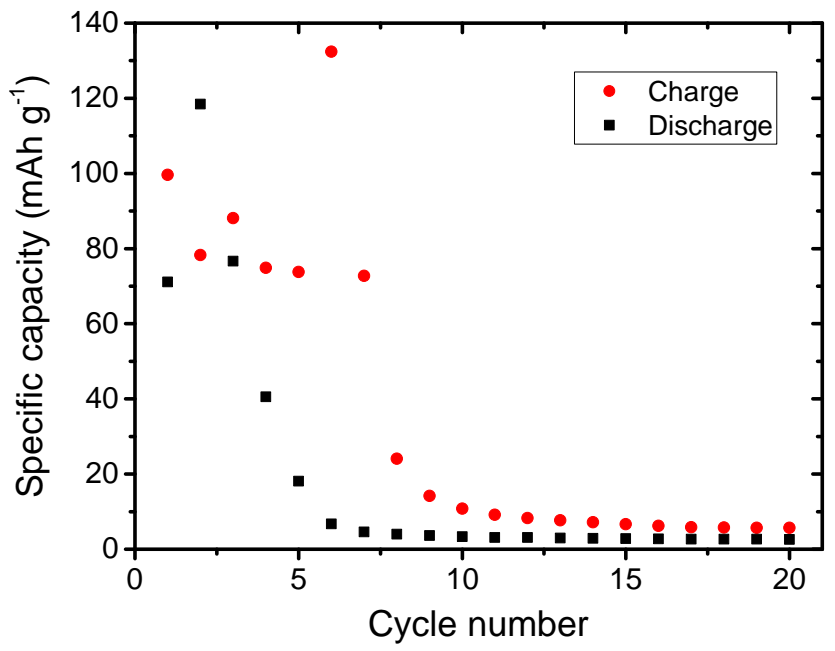

FIGURE A.17: Specific capacities of a typical galvanostatic charge-discharge test (charge first, 20 cycles at a current rate of $100 \mathrm{~mA} \mathrm{~g}^{-1}$ ) of a Swagelok-type cell built using core-sheath $\mathrm{C} / \mathrm{V}_{2} \mathrm{O}_{5}$ nanofibres as cathode. 


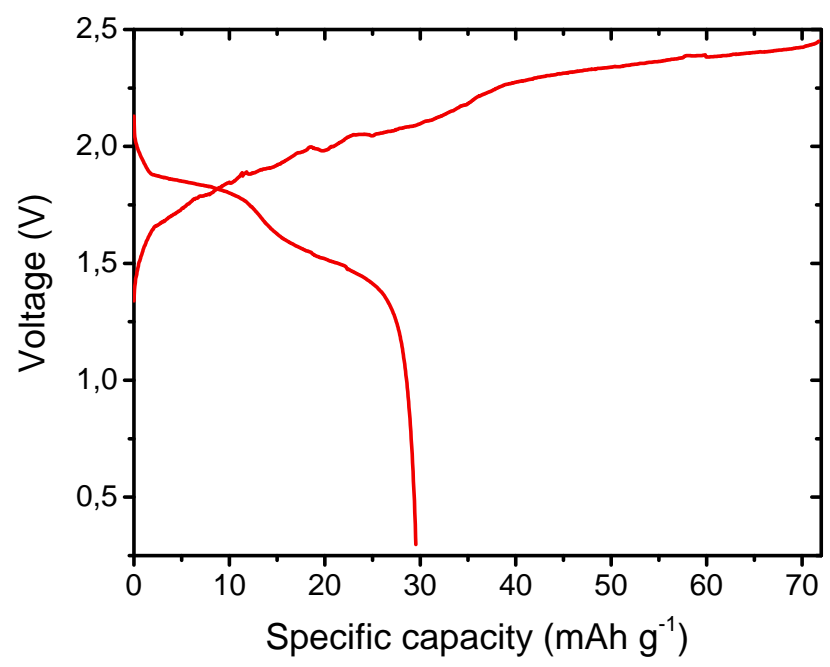

FIGURE A.18: Typical galvanostatic charge-discharge profiles (first cycle, using a flooded three-electrode cell) of an $\mathrm{AcAm} / \mathrm{AlCl}_{3}$ electrolyte with $r=1.3$, using pyrolytic graphite paper as the working electrode. The voltage reported in this plot is the cell potential.

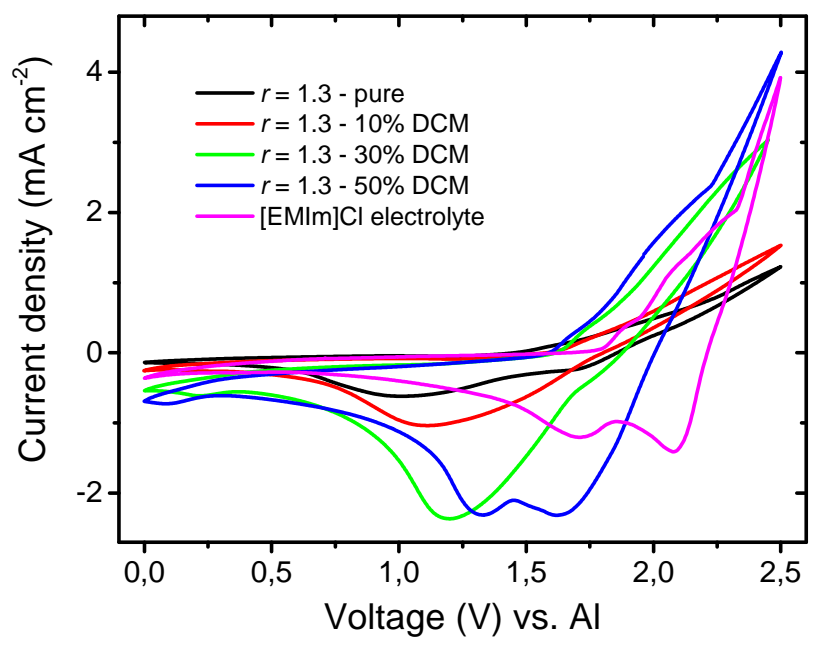

FIGURE A.19: Cyclic voltammograms (third cycle, using a flooded threeelectrode cell) of acetamide- $\mathrm{AlCl}_{3}$ electrolytes with $r=1.3$, diluted with different volume fractions of dichloromethane, compared with the [EMIm]Cl-based electrolyte. The current densities are referred to the projected area of the working electrodes; the voltage is measured against an $\mathrm{Al}$ foil pseudo-reference electrode. 

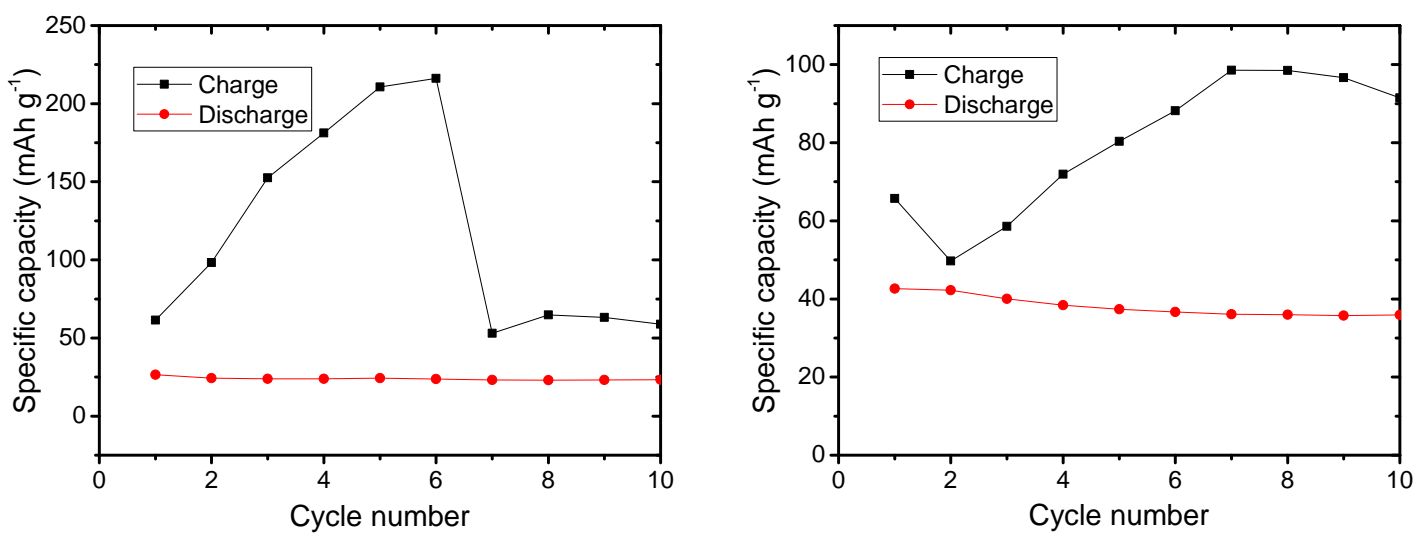

FIGURE A.20: Specific capacities of typical galvanostatic charge-discharge test (10 cycles at a current rate of $50 \mathrm{~mA} \mathrm{~g}^{-1}$ ) of Swagelok-type cells built using diluted AcAm-based electrolytes: $r=1.3+30 \%$ dichloromethane (left) and $r=1.5+50 \%$ dichloromethane (right).

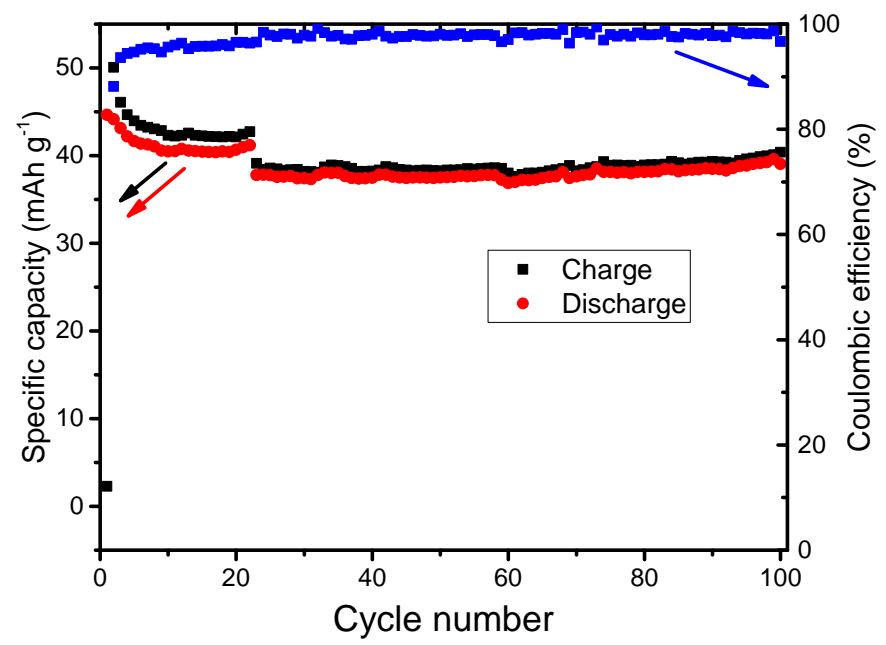

FIGURE A.21: Specific capacities and coulombic efficiencies of a typical galvanostatic charge-discharge test (100 cycles at a current rate of $\left.100 \mathrm{~mA} \mathrm{~g}^{-1}\right)$ of a Swagelok-type cell built using a mixed amide electrolyte (PrAm/AcAm in a $70 / 30$ molar ratio), with an $r$ ratio of 1.5. 